\title{
VISUALIZATION OF DETONATION PROPAGATION IN A ROUND TUBE EQUIPPED WITH REPEATING ORIFICE PLATES
}

by

Georgina Rainsford

A thesis submitted to the Department of Mechanical and Materials Engineering

In conformity with the requirements for

the degree of Master of Applied Science

\author{
Queen's University \\ Kingston, Ontario, Canada
}

(September, 2017)

Copyright @Georgina Rainsford, 2017 


\begin{abstract}
This study used self-luminous high-speed photography to visualize quasi-detonation propagation and deflagration-to-detonation transition (DDT) in a transparent round tube equipped with repeating orifice plates. Experiments were conducted in a combustion channel consisting of a $3.16 \mathrm{~m}$ square channel with a $7.6 \mathrm{~cm}$ by $7.6 \mathrm{~cm}$ cross-section connected to a $1.55 \mathrm{~m}$ cylindrical channel filled with orifice plates. Rectangular 'fence-type' obstacles were installed on the top and bottom of the square channel with a 3.8 $\mathrm{cm}$ opening between them. Two sets of orifice plates with different diameters, $\mathrm{d}$, representing different blockage ratios $(\mathrm{BR})$ were tested $(\mathrm{d}=5.33 \mathrm{~cm}$ for $50 \% \mathrm{BR}$ and $3.81 \mathrm{~cm}$ for $75 \% \mathrm{BR}$ orifice plates). Stoichiometric hydrogen-oxygen mixtures at initial pressures of 4-60 kPa were ignited at one end of the combustion channel. Average propagation velocities were derived from shock-time-of-arrival measurements using pressure transducers in the square channel and high-speed video filmed through the round tube.

As established in literature, a substantially higher orifice-diameter-to-detonation-cell-size ratio $(d / \lambda)$ was observed for the $75 \%$ BR tests $(d / \lambda=14)$ than the $50 \%$ BR tests $(d / \lambda=1.4)$ and Peraldi's $d / \lambda \geq 1$ criterion for the DDT limit. Video footage revealed that this difference may be attributed to a difference in near-limit detonation initiation modes: at $75 \%$ BR, detonation initiation occurred on the orifice face after shock reflection, whereas at $50 \% \mathrm{BR}$, detonation initiation occurred on the tube wall between orifice plates.

Synchronized high-speed video and soot foils allowed for the classification of quasi-detonation propagation modes. At $50 \% \mathrm{BR}$, as initial pressure increased, fast-flame propagation transitioned to singlehot-spot wall ignition detonation to multi-hot-spot wall ignition detonation, and finally to continuous detonation propagation. A similar progression was observed at $75 \% \mathrm{BR}$, except that obstacle face ignition detonations occurred between the fast-flame and single-hot-spot regimes, and continuous detonations were not observed as tube strength concerns limited the maximum initial test pressure.
\end{abstract}


For a continuous detonation, a higher average velocity was observed in the round tube than the square channel, which can be attributed to the effect of wave curvature during detonation diffraction past an obstacle (2D diffraction in the square channel compared to 3D diffraction in the round tube). 


\section{Acknowledgements}

First and foremost, I'd like to thank my supervisor, Dr. Gaby Ciccarelli, for his guidance, support and teaching over the past two years. This degree has been a wonderful opportunity and it has been a real pleasure to work on this project with him.

I am grateful to the McLaughlin technicians (Andy Bryson, Derek Hodgson, Paul Moreland, Corey Fowler, Pat Given and Onno Oosten) who were always generous with their time, patience and jokes. This project would not have been possible without their assistance.

I am particularly grateful to my colleague Mark Kellenberger for showing me around the lab and for his help, practical expertise, and keen photography skills. I also would like to thank Julius Bertato for his contribution of cell-size data to this project.

Finally, I would like to thank my Mum and Dad, Lauren, Alex and friends for their ongoing support, encouragement and often-needed perspective. 


\section{Table of Contents}

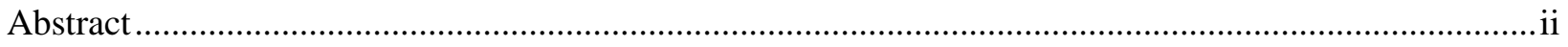

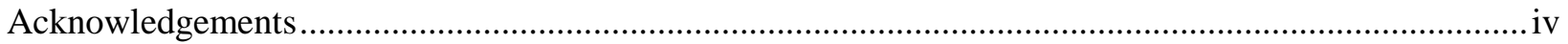

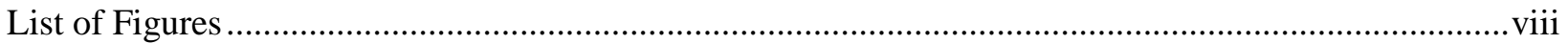

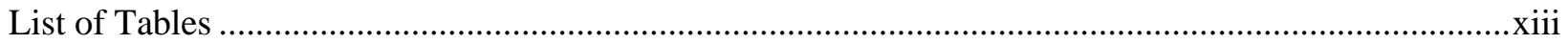

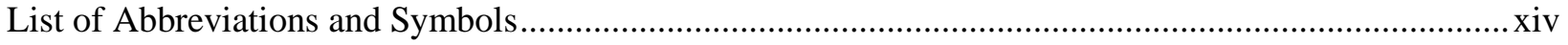

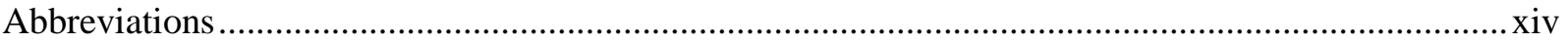

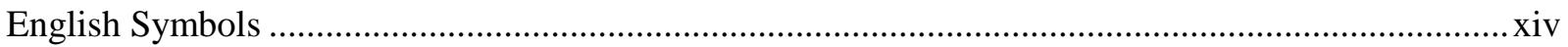

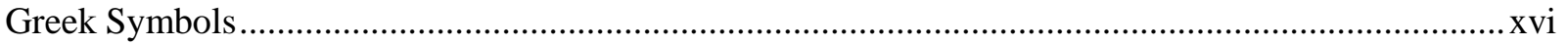

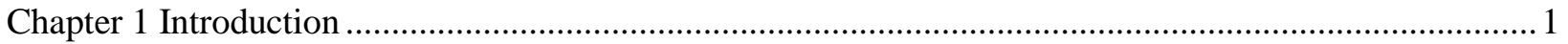

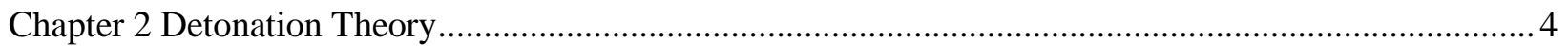

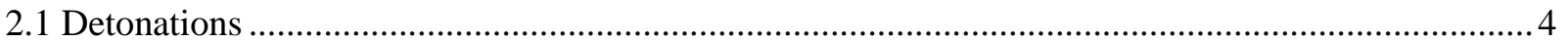

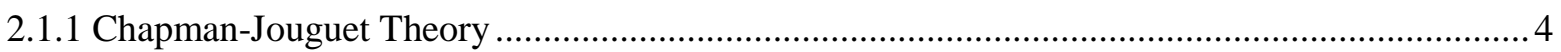

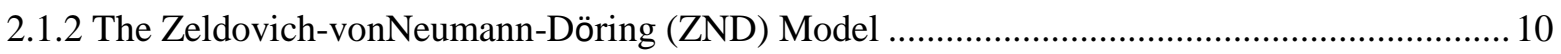

2.1.3 Three-Dimensional Detonation Structure _........................................................................... 13

2.1.4 Soot Foils to Understand Detonation Propagation ............................................................... 16

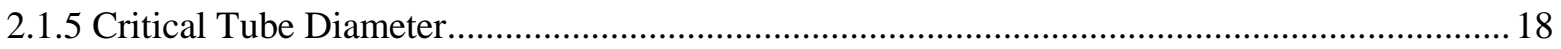

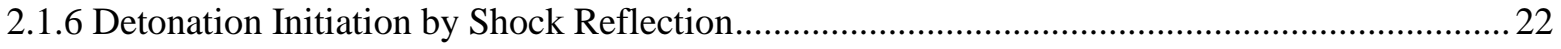

2.2 Deflagration-to-Detonation Transition in a Channel Equipped with Orifice Plates ........................26

2.2.1 Flame Acceleration in an Channel Equipped with Orifice Plates ........................................... 27

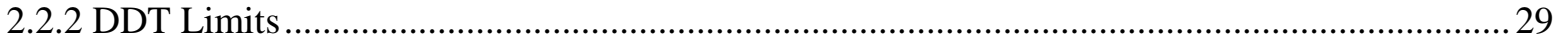

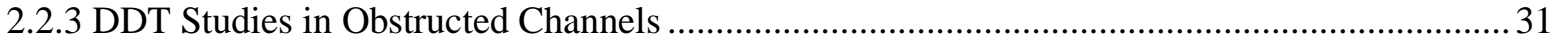

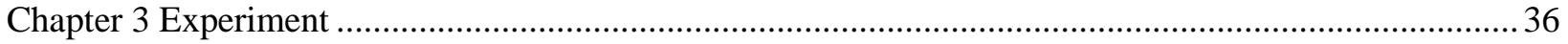

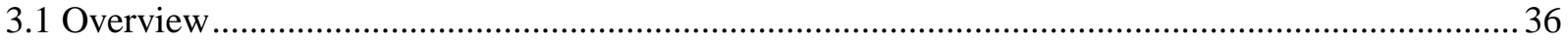

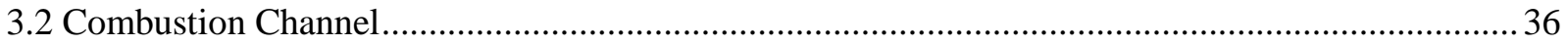

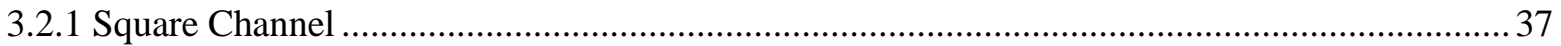

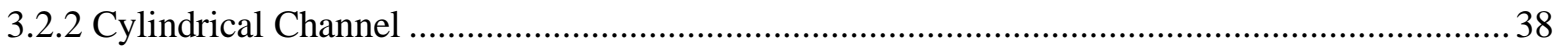

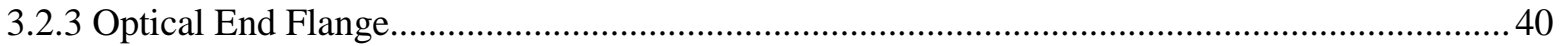

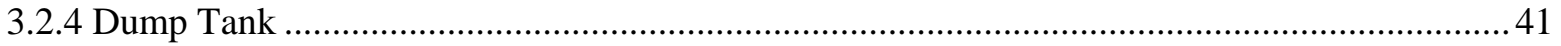

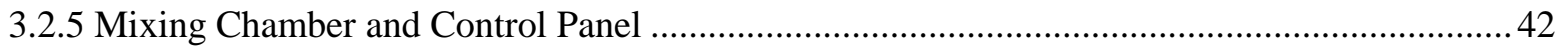

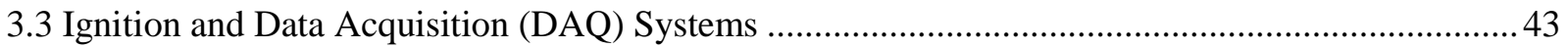

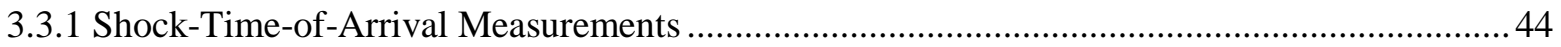

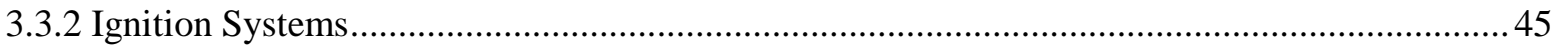




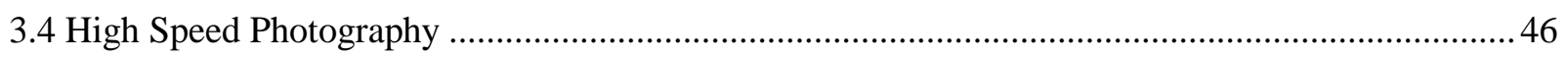

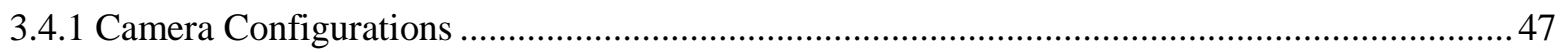

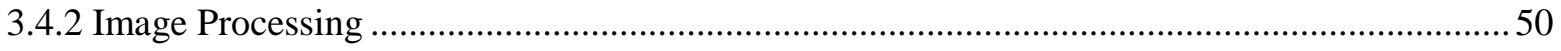

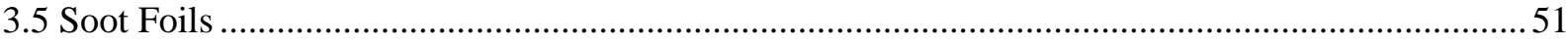

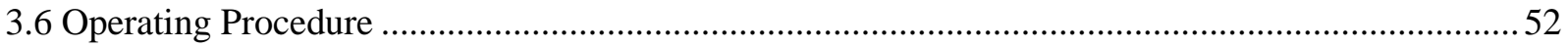

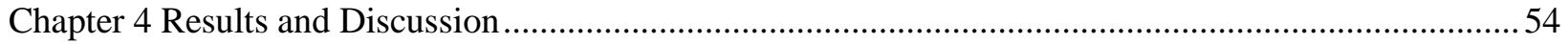

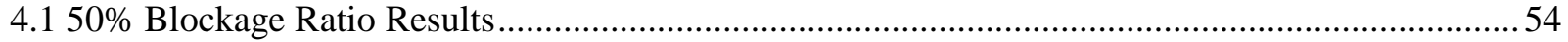

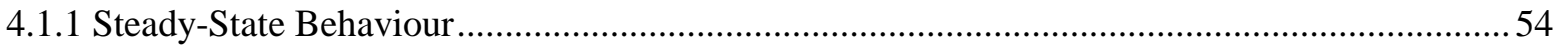

4.1.2 Comparison of Cylindrical and Square Channel Combustion Front Velocities ........................57

4.1.3 Propagation Modes in the Cylindrical Channel .......................................................................59

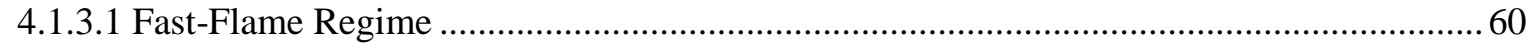

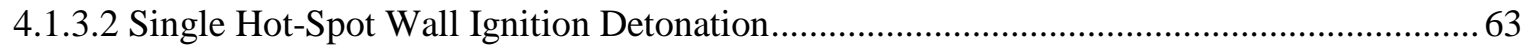

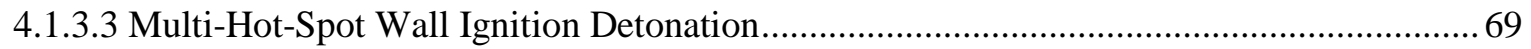

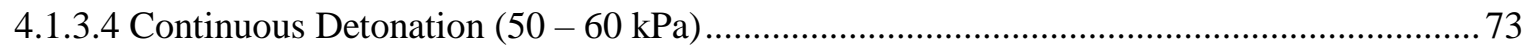

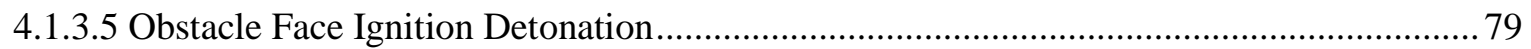

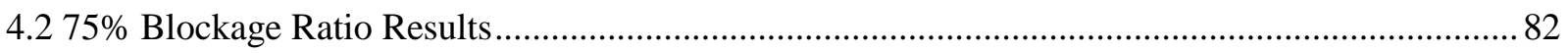

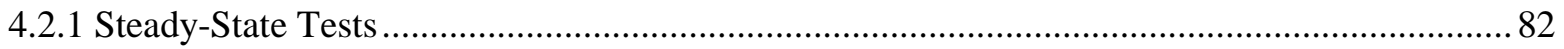

4.2.2 Comparison of Cylindrical and Square Channel Combustion Front Velocities ....................... 84

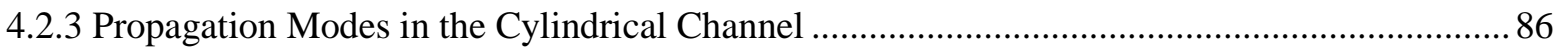

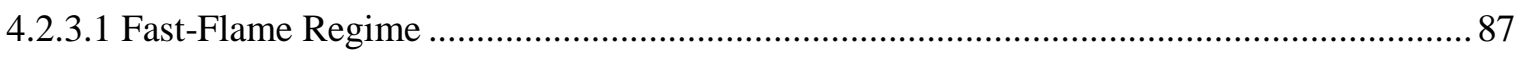

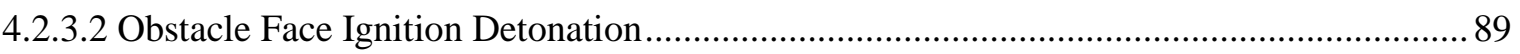

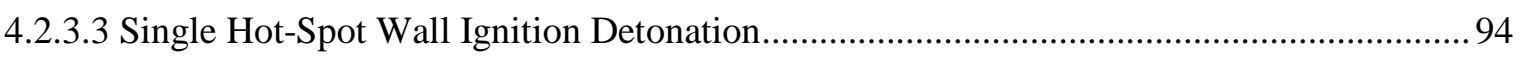

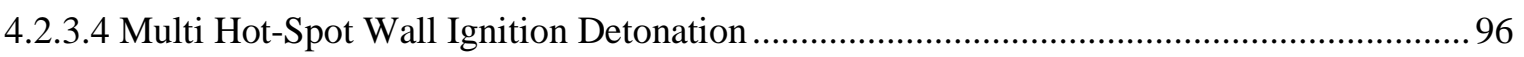

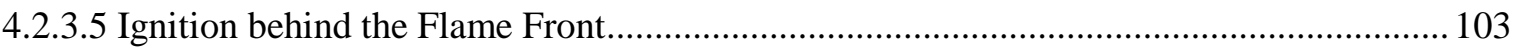

4.2.3.6 Variation in Quasi-Detonation Propagation Modes ........................................................ 107

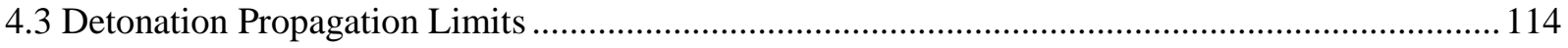

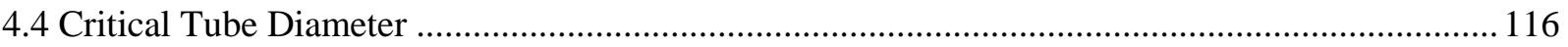

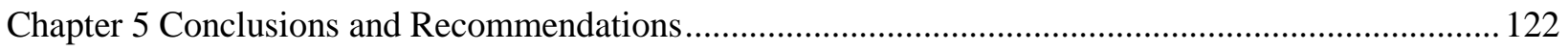

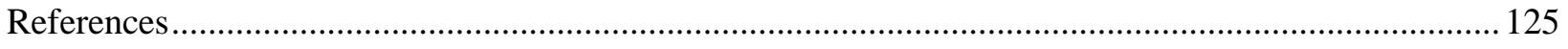

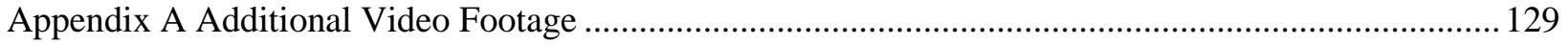

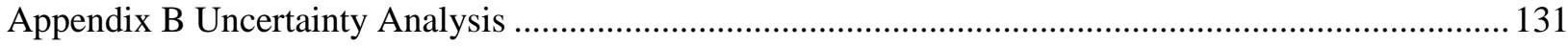

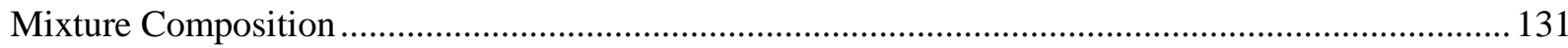

Shock Time of Arrival Velocity Measurements ................................................................................... 132

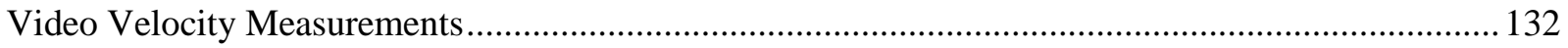




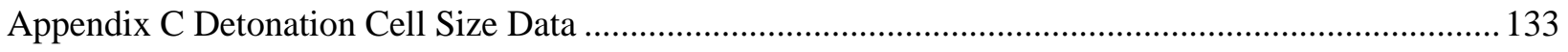

Appendix D Calculated CJ Velocity and Speed of Sound ............................................................... 136 


\section{List of Figures}

Figure 2.1: Schematic of one-dimensional detonation wave [16].The control volume is indicated by a dashed line. The frame of reference is fixed to the combustion wave................................................... 4

Figure 2.2: Plot of Equilibrium Hugoniot and Rayleigh line, adapted from [16] .................................... 7

Figure 2.3: The C-J state located where the Rayleigh line is tangent to the Equilibrium Hugoniot, adapted

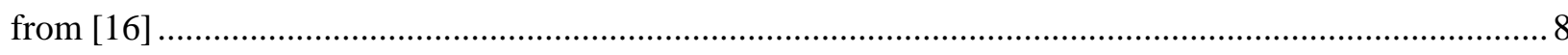

Figure 2.4: Plot of Hugoniot and Rayleigh curves showing tangency on the detonation and deflagration

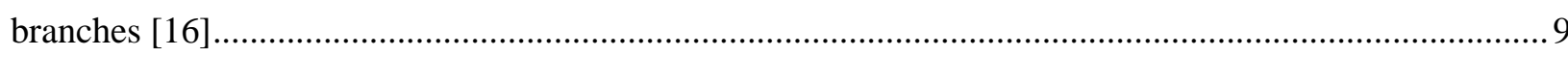

Figure 2.5: Schematic of the ZND detonation wave, overlaid with the CJ control volume. Shown in wave

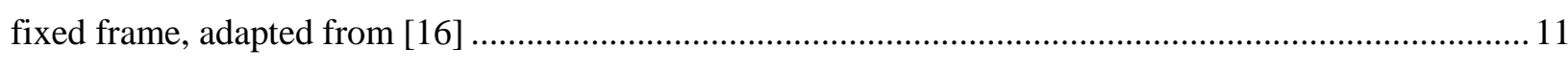

Figure 2.6: Profile of the reaction zone for a stoichiometric-hydrogen air mixture at SATP showing Mach number, temperature and pressure ratio as a function of distance from the shock. The induction zone

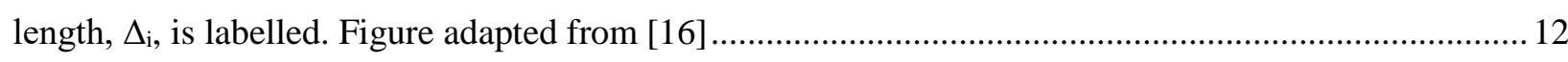

Figure 2.7: Schematic of a triple point showing the transverse wave, incident wave and Mach stem [16].

The arrows indicate propagation directions of the shock waves........................................................... 14

Figure 2.8: A Schlieren photograph showing the corrugated detonation front, adapted from [24] ............ 14

Figure 2.9: Schematic showing an ideal detonation front propagating left to right and its triple point trajectories. Solid dark lines represent shock waves, dotted lines represent the end of the induction zone where recombination reactions start. $\mathrm{L}_{\mathrm{c}}$ shows the characteristic cell length and $\lambda$ indicates detonation cell

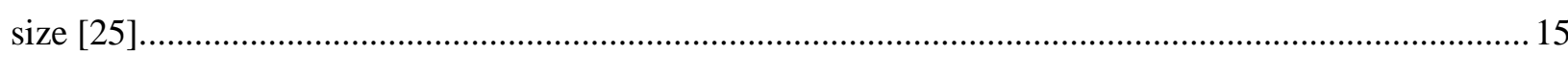

Figure 2.10: Soot foil obtained for a hydrogen-oxygen mixture diluted with argon showing regular

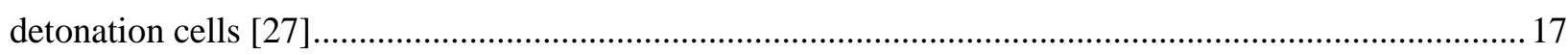

Figure 2.11: Open-shutter photographs of a detonation being transmitted from a channel into an unconfined space showing a) the sub-critical case where the detonation fails completely and $b$ ) the supercritical case where the detonation reinitiates as a cylindrical detonation [30].

Figure 2.12: Sketch of failure and re-initiation mechanism of a detonation emerging from a tube into an unconfined space [30]

Figure 2.13: Plot of critical channel height normalized by detonation cell size as a function of channel aspect ratio [37]....

Figure 2.14: Critical or supercritical case where the detonation begins to fail, but is reinitiated by transverse waves [40]. 
Figure 2.15: Schlieren photograph showing a planar detonation being transmitted into an expansion, subsequent failure of the detonation and then detonation re-initiation upon Mach reflection on the walls [40].

Figure 2.16: Sequence of Schlieren images showing a detonation emerging from a smaller channel and failing completely once through the expansion [40]. 26

Figure 2.17: Schematic showing typical flame acceleration in a channel equipped with repeating orifice

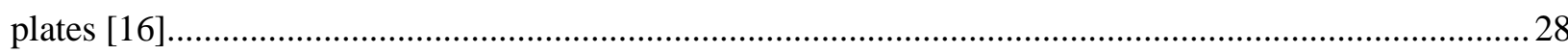

Figure 2.18: Typical flame acceleration profile for flow in an obstructed channel [16] .........................29

Figure 2.19: Plot of DDT limits for hydrogen-air as a function of hydrogen mole fraction [43] ............... 31

Figure 2.20: Soot foil from [26] a stoichiometric hydrogen-air test using a $B R=0.44$ showing two hot spots on the wall and emanating detonation cells. The vertical dashed lines indicate the position of rods

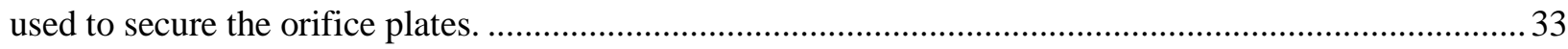

Figure 3.1: Solid Edge model of the combustion channel with the optical end plate installed .................. 37

Figure 3.2: Solid Edge model of the square channel showing the interior cross-section ......................... 37

Figure 3.3: End-view down the square channel showing the opening, d, between the fence-type obstacles .38

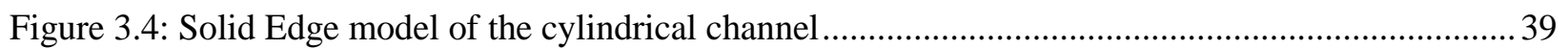

Figure 3.5: Acrylic (left) and aluminum (right) orifice plates for 50\% BR tests ..................................... 40

Figure 3.6: End flange providing optical access down the length of the tube [45].................................. 41

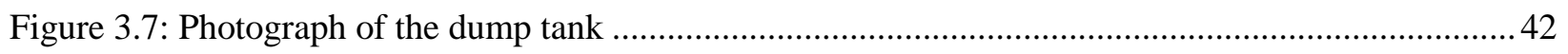

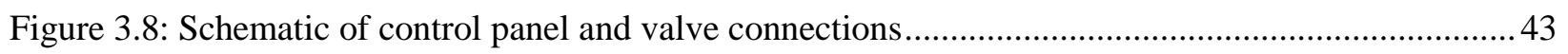

Figure 3.9: Plot of the pressure signals recorded as a function of time used for a $30 \mathrm{kPa}$ test (Test 161).. 45

Figure 3.10: Ignition flange showing the position of the spark and glow plug ignition systems...............46

Figure 3.11: Exploded view of Solid-Edge model showing how the curved and flat foils are installed in

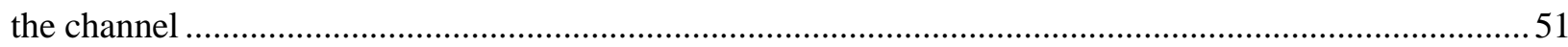

Figure 4.1: Plot of velocity profiles along the cylindrical channel for 50\% BR for a range of initial

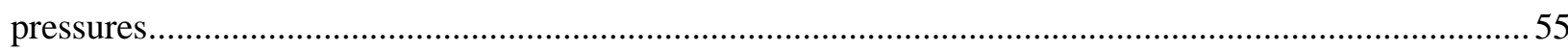

Figure 4.2: Plot of average combustion front velocity in the square and cylindrical channels as a function

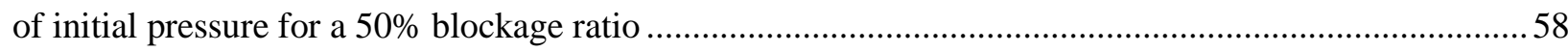

Figure 4.3: Full-view video for a $6.5 \mathrm{kPa}$ test with $50 \% \mathrm{BR}$, showing the fast-flame regime filmed at

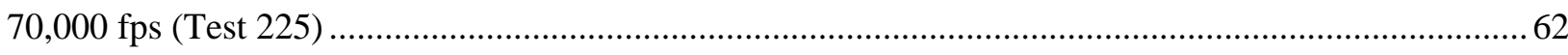

Figure 4.4: Video from a $10 \mathrm{kPa}$ test with 50\% BR (Test 86), filmed at 140,000 fps. Top: Side-view video with field-of-view between OP 8 and 9, wave propagation right to left; Bottom: End-view video ...........64 
Figure 4.5: Curved soot foil obtained for a $10 \mathrm{kPa}$ test with 50\% BR showing a single point of detonation initiation (Test 86). .66

Figure 4.6: Side-view filmed at 300,000 fps, but only every fourth image is presented shown of the single wall hot spot ignition detonation for a $15 \mathrm{kPa}$ test with $50 \%$ BR (Test 192)... .67

Figure 4.7: Schematic showing single hot spot wall ignition detonation mechanism propagating through the obstructed channel. .69

Figure 4.8: Side-view video footage of a $40 \mathrm{kPa}$ test with $50 \% \mathrm{BR}$ showing multiple detonation initiation points on the tube wall, filmed at 300,000 fps (Test 204)... .70

Figure 4.9: Images from a $25 \mathrm{kPa}$ test with a 50\% BR (Test 75) showing detonation initiation from two hot spots on the tube wall, filmed at $140,000 \mathrm{fps}$ .71

Figure 4.10: A curved soot foil for $40 \mathrm{kPa}$ test with $50 \% \mathrm{BR}$, showing multiple detonation initiation

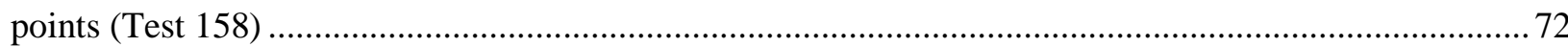

Figure 4.11: Schematic showing multi-hot spot wall ignition detonation propagation mechanism ...........72 Figure 4.12: Side-view video footage of a $60 \mathrm{kPa}$ test with 50\% BR, filmed at 70,000 fps (Test 153) ..... 73 Figure 4.13: Vertical centreline soot foil installed for a $60 \mathrm{kPa}$ test with 50\% BR (Test 157). Circle highlights where triple point interacts with tube wall. .74

Figure 4.14: Video of $60 \mathrm{kPa}$ test with 50\% BR recorded at 175,000 fps (Test 157). The soot foil presented in Figure 4.13 is installed vertically between OP 8 and 9, so the only the front half of the channel is visible. .75

Figure 4.15: x-t diagram generated showing constant velocity over a five obstacle field-of-view for a 60 $\mathrm{kPa}$ test with 50\% BR (Test 137).

Figure 4.16: Still image from a $60 \mathrm{kPa}$ test with $50 \%$ BR, showing ring of detonation 'bubbles' forming on the tube wall, filmed at 70,000 fps (Test 139)

Figure 4.17: A curved soot foil for a $59.8 \mathrm{kPa}$ test showing detonation 'lobes' with 50\% BR (Test 157). 78

Figure 4.18: Schematic showing continuous detonation propagation mode..... .78

Figure 4.19: Images of combustion front propagating from right to left for an $8 \mathrm{kPa}$ test (Test 56), captured at 70,000 fps. The camera is centered on OP 7, as a result the right-face of obstacles 8 and 9 can be seen when illuminated by ignition caused by shock reflection as in image 6 and 9 . The bright white tiny spots seen are burning particles of plastic eroded from the inside surface of the orifice plates. .......... 80 Figure 4.20: Curved soot foil obtained for an $8 \mathrm{kPa}$ test with 50\% BR (Test 89) ................................... 81 Figure 4.21: Schematic showing obstacle face ignition detonation adapted from [43] 82 Figure 4.22: Plot of combustion front velocity profiles along the entire cylindrical channel for various initial pressures $(B R=0.75)$ .83 
Figure 4.23: Plot of average combustion front velocity as a function of initial pressure for $75 \%$ BR in the cylindrical channel and 50\% BR in the square channel.....

Figure 4.24: Side-view footage filmed at 70,000 fps for a $15 \mathrm{kPa}$ test with $75 \%$ BR showing fast-flame

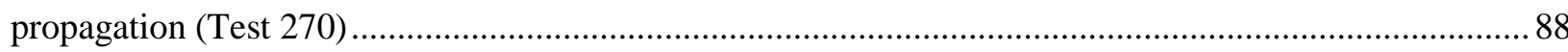

Figure 4.25: Side-view video captured at 70,000 fps for a $40 \mathrm{kPa}$ test with $75 \%$ BR showing obstacle face ignition (Test 247). .90

Figure 4.26: Obstacle face ignition initiated at a single point for a $45 \mathrm{kPa}$ test with $75 \% \mathrm{BR}$ filmed at 300,000 fps (Test 242).......

Figure 4.27: Top: Curved foil obtained for a $50 \mathrm{kPa}$ test with 75\% BR installed between OP 9 and 10 showing detonation initiation off the obstacle (Test 111) Bottom: Close-up of the foil showing the very fine detonation cell structure.

Figure 4.28: Video captured for a $55 \mathrm{kPa}$ test with $75 \% \mathrm{BR}$ showing detonation being initiated at multiple sites on the obstacle face (Test 243) ...

Figure 4.29: Side-view footage filmed at 175,000 fps for a $50.1 \mathrm{kPa}$ test with $75 \% \mathrm{BR}$ (Test 115) showing a detonation forming on OP 8 . .94

Figure 4.30: Side-view video footage captured between OP and for a $50 \mathrm{kPa}$ test with $75 \% \mathrm{BR}$ showing a single point of detonation initiation on the tube wall (Test 117) 95

Figure 4.31: Curved soot foil obtained for a $50 \mathrm{kPa}$ test with $75 \%$ BR installed between Orifice Plates 9 and 10 showing detonation initiation from a single point on the wall (Test 106)... .96 Figure 4.32: Side-view video of a $55 \mathrm{kPa}$ test with $75 \%$ BR showing multiple ignition points on the wall,

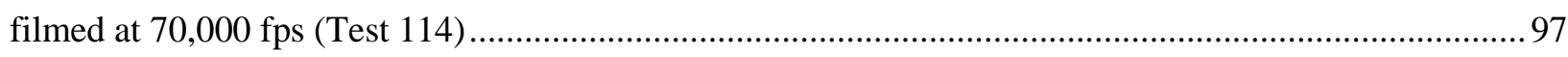

Figure 4.33: Side-view video from a $55 \mathrm{kPa}$ test with 75\% BR, filmed at 300,000 fps (Test 243)...........98

Figure 4.34: Side-view video for a $60 \mathrm{kPa}$ test with 75\% BR captured at 70,000 fps (Test 99) ...............99

Figure 4.35: Close-up of a $60 \mathrm{kPa}$ test with $75 \% \mathrm{BR}$ showing multiple detonation initiation points on the all, filmed at 175,000 fps between Orifice Plates 7 and 8 (Test 105) ... 100

Figure 4.36: Curved soot foil for $60 \mathrm{kPa}$ test with $75 \%$ BR showing multiple detonation initiation points (Test 105) 101

Figure 4.37: A centreline soot foil installed vertically between OP 14 and 15 for a $60 \mathrm{kPa}$ test with 75\%

BR (Test 278). 102

Figure 4.38: Video footage of a $60 \mathrm{kPa}$ test with 75\% BR filmed at 175,000 fps (Test 278) .... 103

Figure 4.39: Side-view of a $30 \mathrm{kPa}$ test with $75 \%$ BR showing detonation occurring at the obstacle face behind the flame front, filmed at 300,000 fps, but presented with an effective frame rate of 100,000 fps Note that the horizontal dotted-lines seen in the images are a result of issues with the camera. (Test 274) 
Figure 4.40: Video filmed at 300,000 fps showing a 'wrap-around detonation' occurring behind the combustion front for a $35 \mathrm{kPa}$ test with $75 \% \mathrm{BR}$ (Test 241).

Figure 4.41: Schlieren images of a $25 \mathrm{kPa}$ stoichiometric hydrogen-oxygen test completed in the square channel showing the turbulent flame structure approaching the obstacle [10]. 106

Figure 4.42: Schematic showing detonation initiation due to turbulent mixing far behind the flame front

Figure 4.43: Plot of average combustion front velocity along the entire round tube for initial pressures

between 50 and $60 \mathrm{kPa}$ showing the variation in propagation speeds ..... 108

Figure 4.44: Side-view filmed at 300,000 fps of a $55 \mathrm{kPa}$ test with $75 \%$ BR showing hot-spot ignition on the wall (Test 243) 110

Figure 4.45: Combustion front velocity profile for a $45 \mathrm{kPa}$ test with $75 \% \mathrm{BR}$ (Test 246) 111 Figure 4.46: Side-view video footage of a $45 \mathrm{kPa}$ test, originally filmed at 300,000 fps presented with an effective frame rate of 200,000 fps (Test 246). 113 Figure 4.47: Comparison plot of average combustion front velocities in the cylindrical channel for $\mathrm{BR}=$ 0.5 and $\mathrm{BR}=0.75$ as a function of initial pressure 115

Figure 4.48: Centreline soot foil obtained from a $30 \mathrm{kPa}$ test with 50\% BR (Test 161). The labeled dashed boxes indicate the relative position of the orifice plates. 118 Figure 4.49: Centreline soot foil obtained from a $40 \mathrm{kPa}$ test with 50\% BR (Test 158). The labeled dashed boxes indicate the relative position of the orifice plates. 119 Figure 4.50: Centreline foil for a $60 \mathrm{kPa}$ test with 50\% BR (Test 157). The labeled dashed boxes indicate the relative position of the orifice plates. 120 Figure 4.51: Centreline foil installed for a $60 \mathrm{kPa}$ test with 75\% BR (Test 278). The dashed boxes indicate the relative positions of the orifice plates 121

Figure 5.1: Test showing hot-spot ignition behind flame front for a $25.1 \mathrm{kPa}$ test with $75 \% \mathrm{BR}$, shown at 150,000 fps (Test 273). 130

Figure 5.2: Plot of average detonation cell size as a function of initial pressure 134 


\section{List of Tables}

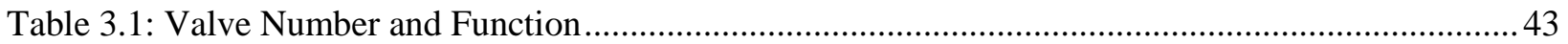

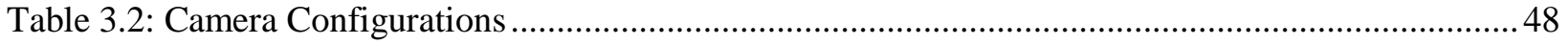

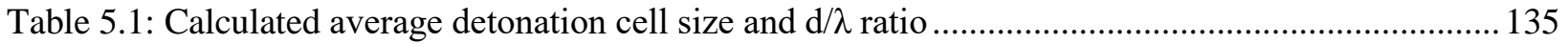

Table 5.2: Calculated CJ Velocity and Products Speed of Sound .......................................................... 136 


\section{List of Abbreviations and Symbols}

\begin{tabular}{ll} 
Abbreviations \\
BR & Blockage Ratio \\
CFD & Computational Fluid Dynamics \\
CJ & Chapman-Jouguet \\
CMOS & Complementary Metal-Oxide-Semiconductor \\
DAQ & Data Acquisition \\
DC & Direct Current \\
DDT & Deflagration-to-Detonation Transition \\
FPS & Frames per second \\
ID & Inner Diameter \\
LED & Light-emitting diode \\
NI & National Instruments \\
OD & Outer Diameter \\
OP & Orifice Plate \\
PT & Pressure Transducer \\
TAT & Trandard Ambient Pressure and Temperature \\
\hline &
\end{tabular}

\section{English Symbols}
A
Arrhenius constant
b
Empirical constant in Arrhenius equation
c
Speed of sound 


\begin{tabular}{|c|c|}
\hline$c_{p}$ & Specific heat (constant pressure) \\
\hline$c_{p}$ & Specific heat (constant volume) \\
\hline $\mathrm{d}$ & Orifice plate diameter and opening between obstacles \\
\hline $\mathrm{D}$ & Tube Diameter \\
\hline$d_{c}$ & Critical tube diameter \\
\hline $\mathrm{E}_{\mathrm{a}}$ & Activation energy \\
\hline $\mathrm{h}$ & Enthalpy \\
\hline$h_{f}{ }^{o}$ & Enthalpy of formation \\
\hline $\mathrm{H}$ & Square channel height \\
\hline $\mathrm{h}_{\mathrm{c}}$ & Critical channel height \\
\hline $\mathrm{k}$ & Ratio of specific heats \\
\hline $\mathrm{L}_{\mathrm{c}}$ & Length of detonation cell \\
\hline M & Mach number \\
\hline $\mathrm{M}_{\mathrm{CJ}}$ & Mach number at $\mathbf{C J}$ state \\
\hline $\mathrm{P}$ & Pressure \\
\hline $\mathrm{P}_{\mathrm{i}}$ & Initial Pressure \\
\hline $\mathrm{px}$ & Pixel distance \\
\hline $\mathrm{q}$ & Heat transfer \\
\hline$\overline{\mathrm{q}}$ & Average heat release \\
\hline $\mathrm{R}$ & Universal Gas Constant \\
\hline $\mathrm{t}$ & Time \\
\hline $\mathrm{T}$ & Temperature \\
\hline $\mathrm{u}$ & Wave velocity \\
\hline $\mathrm{v}_{\mathrm{c}}$ & Average combustion front velocity along channel centerline \\
\hline $\mathrm{w}_{\mathrm{c}}$ & Critical channel width \\
\hline
\end{tabular}




\section{Greek Symbols}

$\begin{array}{ll}\alpha & \text { Pixel density } \\ \Delta & \text { ZND reaction zone length } \\ \Delta_{\mathrm{i}} & \text { Induction zone length } \\ \lambda & \text { Detonation cell width } \\ \rho & \text { Density }\end{array}$




\section{Chapter 1}

\section{Introduction}

There are two types of self-propagating combustion waves: deflagrations (more commonly known as flames) and detonations. Most engineering applications involving combustion employ the deflagration mode, e.g. burners and combustors. Deflagrations propagate at subsonic speeds with a slight drop in pressure across the wave. Propagation of a laminar deflagration occurs via the diffusion of mass and heat across the flame front, and therefore the propagation speed is governed by the mixture transport properties. The propagation speed can be significantly enhanced by turbulence. For example, in stoichiometric gaseous fuel-air mixtures, a laminar deflagration propagates at approximately $1 \mathrm{~m} / \mathrm{s}$, while a turbulent deflagration can propagate at several hundreds of metres per second. In a laterally confined space, such as a channel equipped with repeating orifice plates, a deflagration is often preceded by a leading shock.

A detonation wave travels at a supersonic speed and consists of a chemical reaction zone coupled to a lead shock wave. Combustion occurs via adiabatic shock heating, where the shock compresses the reactants generating high temperature and pressure that triggers auto-ignition of the compressed reactants. In stoichiometric fuel-air mixtures, detonations travel at thousands of metres per second and produce a very high dynamic overpressure (approximately 20 bars). For these reasons, detonations pose a greater threat to industrial safety than deflagrations and have prompted research interest for hundreds of years.

Due to the large amount of energy required to directly initiate a detonation, detonations usually develop from a deflagration [1]. With the presence of turbulence generating obstacles, a deflagration can accelerate, and under certain conditions, a deflagration-to-detonation transition (DDT) can occur.

Historically, the driving force behind detonation research has always been a desire to improve industrial safety. In 1883, Mallard and Le Chatelier [1], authors of the original detonation studies, were concerned with the risk of uncontrolled explosions in coal mines. Coal mines allowed air, methane gas and volatile hydrocarbons to mix, forming a highly combustible mixture which could be ignited by an accidental 
spark. Moreover, the structure of the mine shaft provided ideal conditions for a flame to accelerate down the shaft, and potentially undergo DDT. The 1979 accident at the Three Mile Island Nuclear plant generated greater investment in DDT and detonation research as fears grew that had DDT occurred, the nuclear containment system could have been compromised leading to nuclear radiation exposure. More recently, the 2005 Buncefield Fuel Depot explosion demonstrated that even natural features in an open-air scenario, such as a grove of trees, can facilitate DDT.

Although still the primary concern of the field, the study of detonations has evolved past its origins in industrial safety. In attempts to take advantage of the nearly constant volume efficiency of the detonation process (compared to the constant pressure deflagration process) for propulsion purposes, Pulse Detonation Engines (PDEs) use controlled detonations to generate thrust [2].

Detonation propagation and DDT have been studied in round tubes equipped with repeating orifice plates since the first flame acceleration studies conducted by Chapman and Wheeler in 1927 [3]. Detonation propagation has been studied extensively in opaque round metal tubes equipped with orifice plates using non-optical diagnostic techniques, with the exception of one study that used a clear Plexiglas tube [4]. In this set-up, detonation propagation has been primarily classified according to average combustion front velocity derived from time-of-arrival measurements taken at regular intervals along the combustion channel [5]. More recently, sooted foils, which provide information on detonation structure, have been used inside the combustion channel between obstacles to better understand detonation propagation [6].

Various visualization studies [7] [8] [9] [10] have been undertaken using Schlieren photography through glass windows in rectangular channels equipped with repeating obstacles. Schlieren photography, which requires collimated light, cannot be performed in a round tube as the curvature of the tube distorts the light. However, even traditional high-speed photography has not been completed in round tubes equipped with repeating orifice plates.

The primary objective of this research is to use self-luminous high-speed photography to visualize detonation propagation in a transparent round tube equipped with repeating orifice plates. To capture the 
combustion phenomenon, two synchronized, high-speed CMOS cameras were used, with supplemental soot foils for some tests. Two sets of orifice plates were used to compare the effect of area blockage ratio and orifice opening size. The effect of blockage ratio on the onset of DDT has been established [11] (more reactive mixtures are required for the onset of DDT at higher blockage ratios) but has not been explained. By conducting a visualization study, differences in the initiation or propagation mechanisms of quasidetonations may be observed, which may account for the effect of blockage ratio on DDT. A secondary objective is to compare the effect of the channel cross-section geometry (square versus round) on detonation propagation, on the same apparatus. 


\section{Chapter 2}

\section{Detonation Theory}

\subsection{Detonations}

From a one-dimensional control volume analysis performed in the 1890s, our understanding of detonation wave structure has evolved to today, where it has been established that detonations are actually complex and highly three-dimensional structures.

\subsubsection{Chapman-Jouguet Theory}

Chapman [12] and Jouguet [13] independently developed a method for calculating the velocity of a detonation front. Built upon Rankine [14] and Hugoniot's [15] analysis of non-reacting gases, the Chapman-Jouguet (CJ) Theory was based on solving the conservation of mass, momentum and energy equations for a defined control volume. By ascribing a control volume with an inlet and outlet sufficiently far up- and down-stream of the combustion wave, Chapman and Jouguet modelled detonations as onedimensional compressible flow. Their model assumed steady-state flow within the control volume and that energy release from combustion occurred instantaneously at the shock wave. As seen in Figure 2.1, State 1 refers to the reactants entering the control volume and State 2 refers to the combustion products exiting the control volume. The model assumes inviscid, steady flow, with no heat loss through a constant area channel and that State 2 is at chemical equilibrium.

$$
P_{2}, \rho_{2}, T_{2} \stackrel{u_{2}}{\hdashline} P_{1}, \rho_{1}, T_{1}
$$

Figure 2.1: Schematic of one-dimensional detonation wave [16]. The control volume is indicated by a dashed line. The frame of reference is fixed to the combustion wave. 
The derivation of the $\mathrm{CJ}$ velocity begins with the steady-state, one-dimensional, inviscid conservation equations, given in Equations 2.1, 2.2 and 2.3.

Conservation of mass:

$$
\rho_{1} u_{1}=\rho_{2} u_{2}
$$

Conservation of momentum:

$$
P_{2}-P_{1}=\rho_{1} u_{1}\left(u_{1}-u_{2}\right)
$$

Conservation of energy:

$$
\frac{1}{2} u_{1}^{2}+h_{1}=\frac{1}{2} u_{2}^{2}+h_{2}
$$

where the enthalpy, $\mathrm{h}$, is defined as

$$
h=h_{f}^{\circ}+c_{p}\left(T-T^{\circ}\right)
$$

Ideal gas behaviour, governed by the ideal gas equation of state (Equation 2.5) is assumed.

$$
P=\rho R T
$$

The heat release, $\mathrm{q}$, written as

$$
q=h_{f 1}^{\circ}-h_{f 2}^{\circ}
$$

can be combined with Equations 2.4 and 2.5, allowing the conservation of energy equation to be re-written as

$$
\frac{1}{2} u_{1}^{2}+c_{p 1} T_{1}+q=\frac{1}{2} u_{2}^{2}+c_{p 2} T_{2}
$$

Assuming constant specific heat, i.e. 


$$
k=k_{1}=k_{2}=\frac{c_{p}}{c_{v}}
$$

and combining the conservation of mass and energy equations (Equations 2.1 and 2.7, respectively), yields the Hugoniot equation (Equation 2.9). Note that not all intermediate mathematical steps are shown. For a given heat release, the Equilibrium Hugoniot equation represents all possible equilibrium end-states (State 2).

$$
c_{p} T_{2}-\left(c_{p} T_{1}+q\right)=\frac{1}{2}\left(P_{2}-P_{1}\right)\left(\frac{1}{\rho_{1}}+\frac{1}{\rho_{2}}\right)
$$

The Rayleigh equation (Equation 2.10) is obtained by combining the conservation of mass (Equation 2.1) and momentum equations (Equation 2.2). When plotted on a pressure-volume plot, the Rayleigh equation represents the thermodynamic path the reactants (at State 1) follow to become products (at State 2) across the combustion wave, for a given wave front velocity $\left(\mathrm{u}_{1}\right)$. The Rayleigh line (Equation 2.10) represents a straight line with the pressure ratio and the inverse density ratio as variables. For a given initial state, the slope of the Rayleigh line is proportional to the square of the wave velocity.

$$
\frac{P_{2}}{P_{1}}=-\left(\frac{\rho_{1} u_{1}^{2}}{P_{1}}\right) \frac{\rho_{1}}{\rho_{2}}\left(1+\frac{\rho_{1} u_{1}^{2}}{P_{2}}\right)
$$

Given the initial conditions $\left(\mathrm{P}_{\mathrm{o}}, \rho_{\mathrm{o}}, \mathrm{T}_{\mathrm{o}}\right)$, there are not enough equations (Equations 2.1, 2.2, 2.3 and 2.4) to solve the five unknowns $\left(\mathrm{u}_{1}, \mathrm{P}_{2}, \rho_{2}, \mathrm{~T}_{2}\right.$, and $\left.\mathrm{u}_{2}\right)$. Chapman and Jouguet differed in their approach to this problem, but both considered the Hugoniot and Rayleigh lines. By necessity, the end-state (State 2), must satisfy both the Hugoniot and Rayleigh equations. This is graphically represented as the intersection of the Equilibrium Hugoniot and Rayleigh lines. For most given front velocities $\left(\mathrm{u}_{1}\right)$, this intersection occurs at two points, labelled State 2 and 2' in Figure 2.2. State 2 represents the strong detonation case and State 2' represents the weak detonation case. 


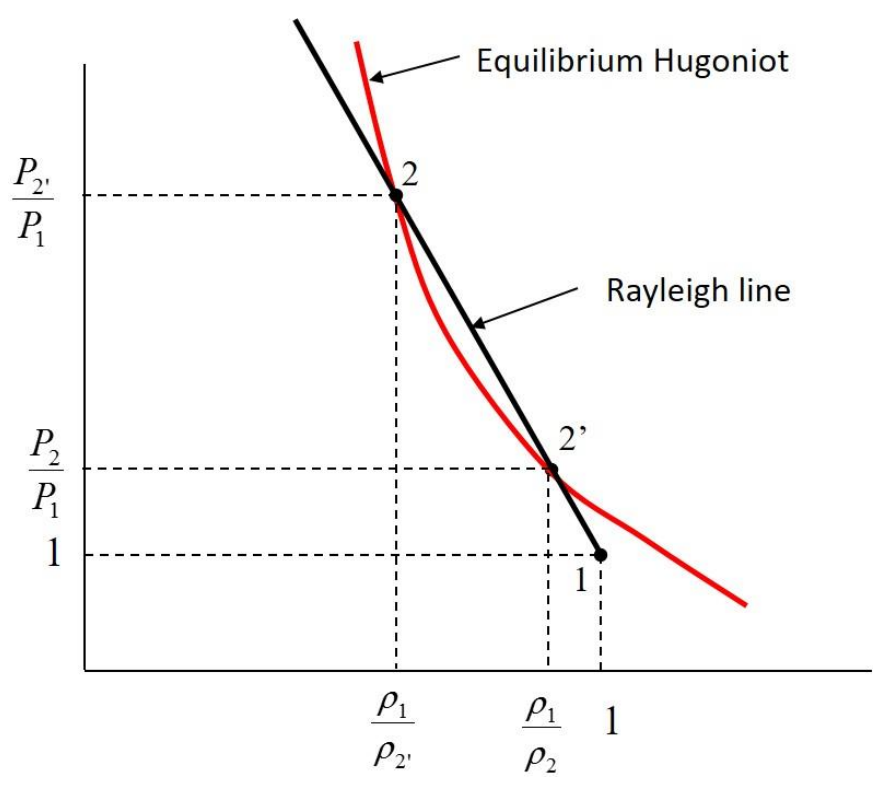

Figure 2.2: Plot of Equilibrium Hugoniot and Rayleigh line, adapted from [16]

The strong detonation case occurs where the outlet flow of the products is subsonic relative to the wave. Ultimately, this case is not stable as the expansion waves generated at the back wall of a tube (necessary because of the zero velocity restriction) will "catch-up" to and weaken the lead shock wave of the detonation front. The case of the weak detonation intersection (State 2'), where the outlet flow is supersonic relative to the detonation wave, is impossible because it represents a decrease in entropy from State 1 to 2', violating the Second Law of Thermodynamics.

There is a unique solution (one value of $\mathrm{u}_{1}$ ) where the Rayleigh line is tangent to the Hugoniot curve. This point, shown in Figure 2.3, is referred to as the CJ state. Although, Chapman and Jouguet made different observations about the CJ state, they both concluded that the tangency condition yields the detonation wave velocity (also known as the CJ velocity). Their conclusion was corroborated using experimental data available at the time. 


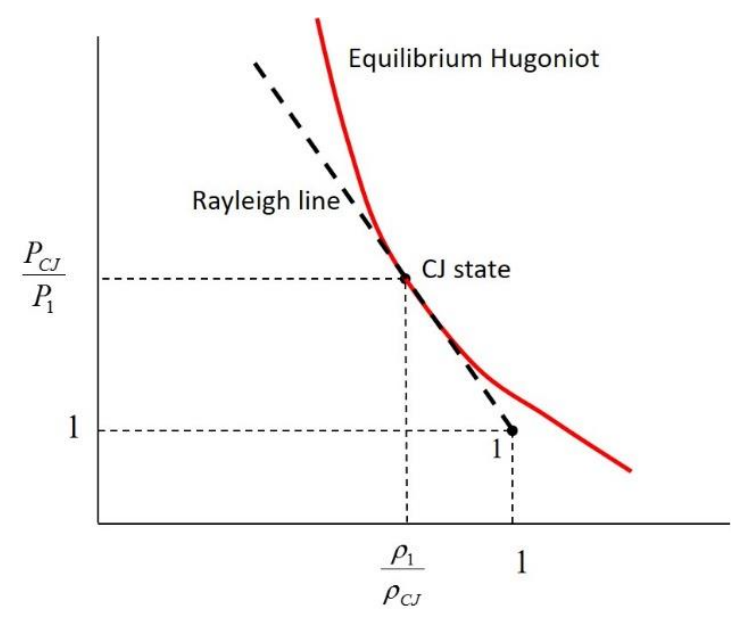

\section{Figure 2.3: The C-J state located where the Rayleigh line is tangent to the Equilibrium Hugoniot, adapted from [16]}

Chapman observed that the $\mathrm{CJ}$ state corresponds to the minimum detonation velocity, known as the CJ velocity, as the Rayleigh and Hugoniot curves do not intersect for velocities less than the CJ velocity. He argued that this must be the detonation velocity as it was experimentally demonstrated that there exists a single detonation velocity for a given mixture and set of initial conditions. Jouguet observed that the CJ state corresponds to sonic flow in the products $\left(\mathrm{u}_{2}\right)$ relative to the detonation wave. Jouguet also observed that this state corresponds to the minimum entropy increase from State 1 to State 2.

There are actually two points where the Rayleigh Line is tangent to the Hugoniot curve, as seen in Figure 2.4. Tangency occurs at one point on the upper and on the lower branches. The upper branch represents states with a pressure increase (pressure ratio across the wave greater than 1), and the lower branch represents a pressure drop (pressure ratio across the wave less than one). Therefore, the upper and lower branches represent the detonation and deflagration modes; and the CJ tangency points represent the minimum detonation velocity and the maximum deflagration velocity, respectively. 


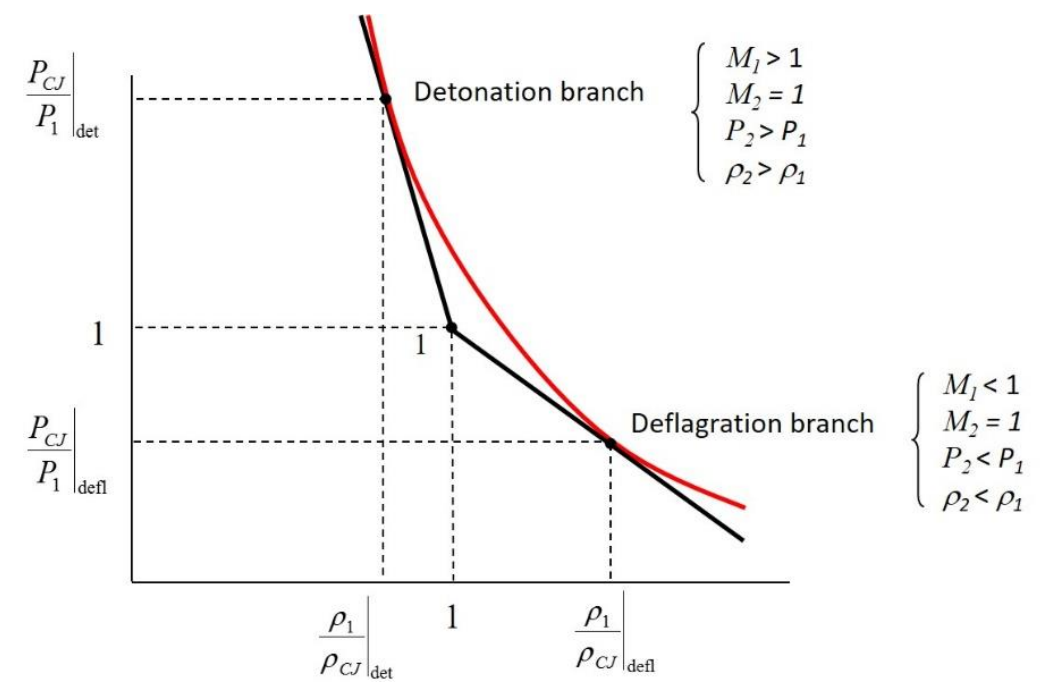

Figure 2.4: Plot of Hugoniot and Rayleigh curves showing tangency on the detonation and deflagration branches [16]

To solve for the CJ velocity, the heat release term, $\mathrm{q}$, is first transformed to a per unit mass basis by normalizing with the square of the fresh gas speed of sound, i.e. by Equation 2.11.

$$
\bar{q}=\frac{q}{c_{1}^{2}}
$$

The CJ velocity is obtained via an equation arrived at by equating the pressure ratio $\left(\mathrm{P}_{2} / \mathrm{P}_{1}\right)$ in the Rayleigh (Equation 2.10) and Hugoniot (Equation 2.9) equations, and incorporating Equation 2.11. This combination allows the density ratio to be determined according to Equation 2.12.

$$
\frac{\rho_{1}}{\rho_{2}}=\frac{1}{k+1}\left(k+\frac{1}{M^{2}}\right) \pm \sqrt{\left(\frac{1}{M_{1}^{2}}-1\right)^{2}-\frac{2\left(k^{2}-1\right) \bar{q}}{M_{1}^{2}}}
$$

The unique solution, corresponding to the CJ State, is obtained when the square root term in Equation 2.12 equals zero. Rearranging, to solve for the Mach number at the $\mathrm{CJ}$ state, $\mathrm{M}_{\mathrm{CJ}}$, gives Equation 2.13. 


$$
\frac{1}{M_{C J}^{2}}=\left(k^{2}-1\right) \bar{q}\left(1+\frac{1}{\left(k^{2}-1\right) \bar{q}} \pm \sqrt{1+\frac{2}{\left(k^{2}-1\right) \bar{q}}}\right)
$$

There are two different solutions to Equation 2.13. The solution generated using the positive term yields the minimum detonation velocity and the solution generated using the negative term yields the maximum deflagration velocity, shown in Figure 2.4.

Although the CJ Theory accurately predicts detonation front velocity, it does not account for the finite time-scale in which combustion occurs. This limitation was addressed by the ZND model.

\subsubsection{The Zeldovich-vonNeumann-Döring (ZND) Model}

In the 1940s, Zeldovich [17], von Neumann [18] and Döring [19] (ZND) independently developed a more advanced model of a detonation wave. Known as the ZND model, their model gave structure to Chapman-Jouguet's black box control volume (shown in Figure 2.1). The steady, one-dimensional ZND model consists of a lead shock wave coupled to a reaction zone ending at the CJ plane.

Figure 2.5 shows a schematic of the ZND detonation model, with the Chapman-Jouguet control volume outlined by a dashed line for comparison. In the ZND model, the inlet to the control volume (State 1) is a lead shock, travelling at the CJ detonation velocity. The outlet of the control volume (State 2) is the CJ-plane, at which point the flow is sonic relative to the lead shock, and chemical equilibrium is achieved. A finite, inviscid reaction zone, where energy release occurs, exists between the shock and CJ-plane where all the energy release occurs. Theoretically, the choked condition is approached asymptotically; however, practically the end of the reaction zone is defined at the point of maximum rate of energy release. 


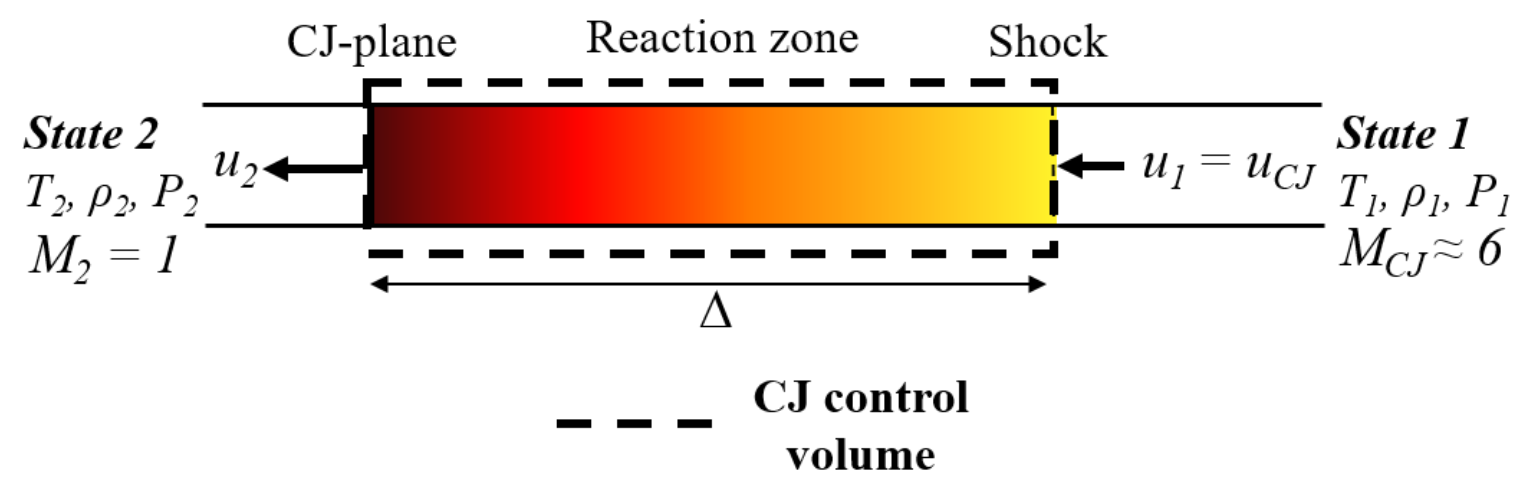

Figure 2.5: Schematic of the ZND detonation wave, overlaid with the CJ control volume. Shown in wave fixed frame, adapted from [16]

For most fuel-air mixtures with high activation energy the reaction zone consists of a long induction zone coupled to a relatively short recombination zone. The induction zone is a constant temperature and pressure region immediately following the lead shock where free radicals are formed. The recombination zone is the region where the free radicals combine to form the final products of combustion. Due to the exothermic reactions occurring, the temperature increases over the recombination zone to reach equilibrium temperature at the CJ plane. A typical temperature profile over the reaction zone is shown in Figure 2.6. The length of the induction zone, $\Delta_{\mathrm{i}}$, is usually defined as the distance from the lead shock to the point of maximum temperature gradient. 


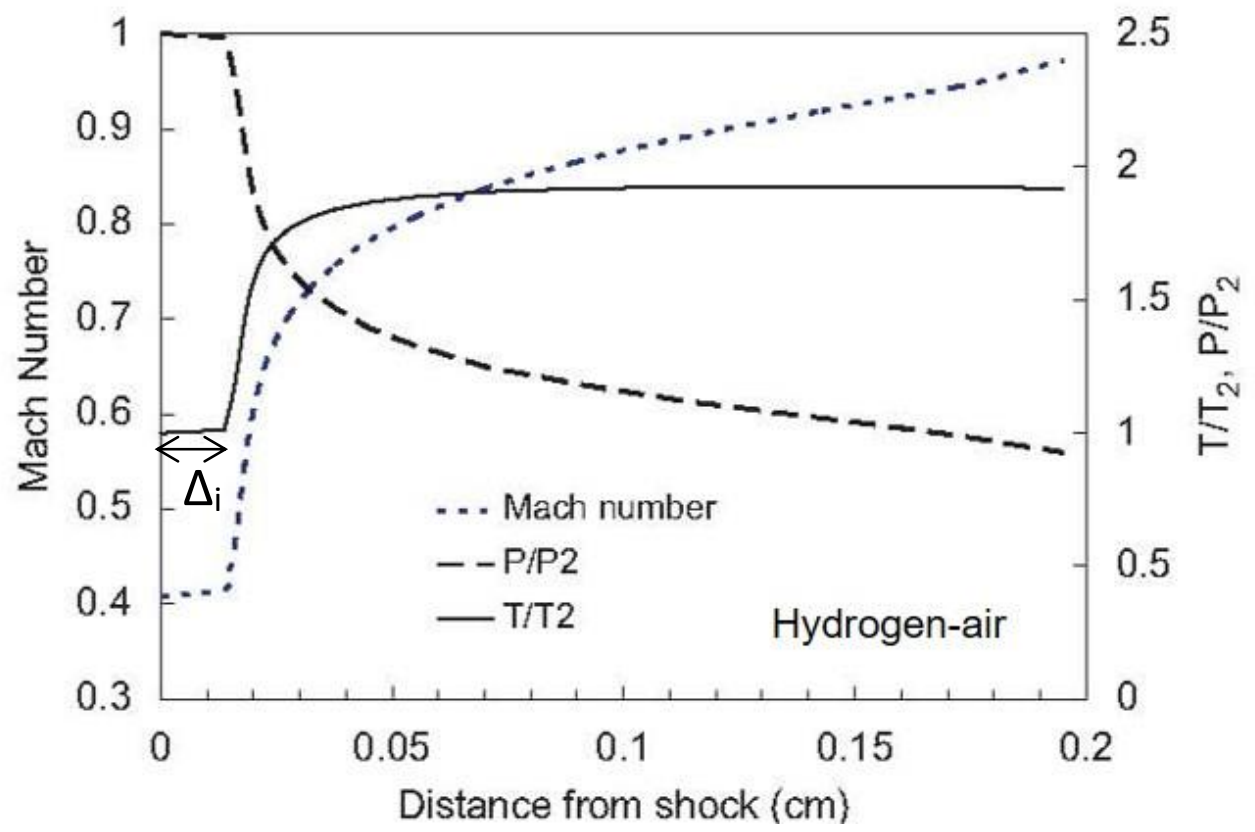

Figure 2.6: Profile of the reaction zone for a stoichiometric-hydrogen air mixture at SATP showing Mach number, temperature and pressure ratio as a function of distance from the shock. The induction zone length, $\Delta_{i}$, is labelled. Figure adapted from [16]

The ZND induction zone length and temperature, pressure and velocity profiles over the reaction zone can be calculated. This is achieved by combining the conservation equations with an equation that describes the rate of chemical reaction. The reaction rate is proportional to the product of the fuel and oxygen concentration multiplied by a rate constant that takes on the Arrhenius form (shown in Equation 2.14),

$$
k=A T^{b} \exp \left(-\frac{E_{a}}{\bar{R} T}\right)
$$

The empirical constants $\mathrm{A}, \mathrm{b}$, and $\mathrm{E}_{\mathrm{a}}$ are defined for the elementary reactions in a detailed chemical reaction mechanism. The reaction zone profile shown in Figure 2.6 was numerically generated using the 'Shock and Detonation Toolbox' developed by Shepherd et al. at the California Institute of Technology [20]. 
The CJ detonation velocity represents the theoretical maximum velocity that a detonation propagates at, uninfluenced by boundary conditions. Early into detonation research, it was experimentally shown that a detonation wave propagating in a channel does not travel at the CJ detonation velocity. Experiments showed that the maximum detonation velocity was typically $1-2 \%$ below the CJ detonation velocity in large-diameter, smooth tubes and that the velocity deficit is inversely proportional to initial pressure and combustion channel diameter [2].

In 1940, Zeldovich suggested that the experimentally measured detonation velocity deficit was due to momentum and heat loss to the channel wall from the reaction zone [17]. This means that the velocity deficit is proportional to the reaction zone length. Therefore, factors that increase the reaction zone length, such as reducing the initial pressure of the mixture, lead to a greater velocity deficit. Heat and momentum loss are wall effects, therefore the detonation velocity deficit decreases with the channel transverse length scale, e.g., diameter for a circular tube. Zeldovich proposed that the velocity deficit is proportional to the wall drag divided by the momentum flux. In 1959, Fay [21] developed a more correct model for the velocity deficit, using boundary layer effects. He modelled the flow in the reaction zone as a quasi-1D, slow moving flow in a diverging channel.

\subsubsection{Three-Dimensional Detonation Structure}

The steady, one-dimensional CJ model was successful in predicting the detonation velocity within the experimental error of the time. But by the late 1950s and early 1960s, growing scientific evidence showed that detonation wave structure was actually three-dimensional, and locally transient. White's interferometry images of a detonation wave uncovered turbulence behind the detonation front [22]. Using the soot foil technique, Denisov and Troshin [23] showed that the detonation front was composed of a leading shock wave with shock waves propagating in the transverse direction. They proposed that the transverse shock waves define triple-points that traverse across the detonation front. The triple-points represent the confluence of three shock waves (the Mach stem, the incident wave and the transverse wave) and a stream line, as discovered by Ernst Mach and shown in Figure 2.7. 


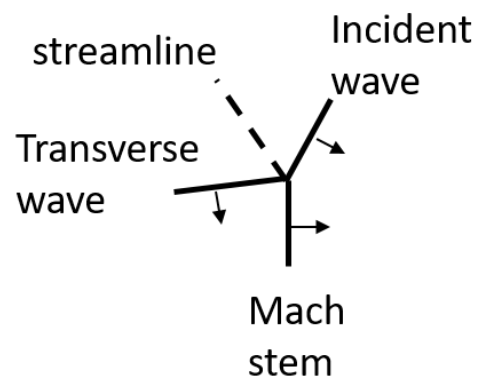

Figure 2.7: Schematic of a triple point showing the transverse wave, incident wave and Mach stem [16]. The arrows indicate propagation directions of the shock waves.

It is now well established that a detonation wave is composed of triple-points propagating in alternating directions along a two dimensional front. This structure can be seen in a Schlieren photograph, shown in Figure 2.8, taken of a detonation wave propagating in a narrow channel. The reaction zone, which exists behind the shock waves moving in the overall direction of detonation propagation (i.e. the incident shock and Mach stem in Figure 2.7), is also visible in the Schlieren image.

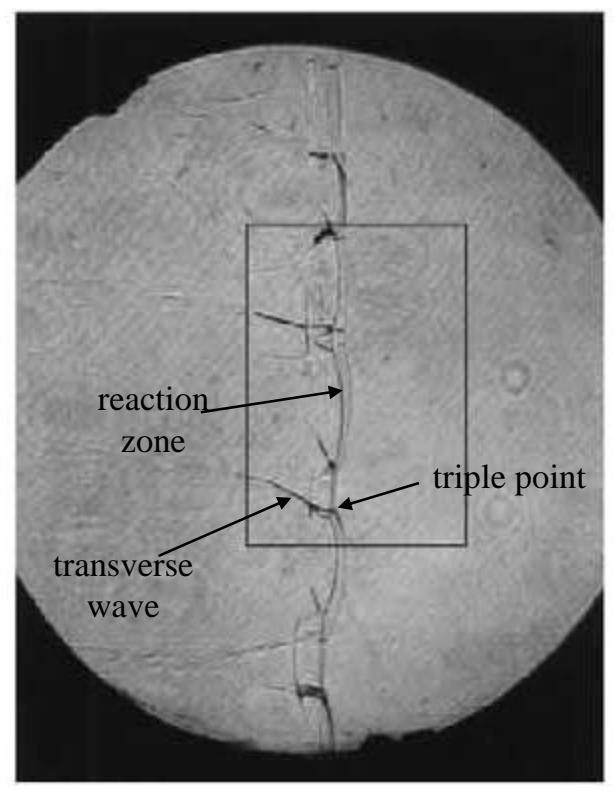

Figure 2.8: A Schlieren photograph showing the corrugated detonation front, adapted from [24] 
The mechanism behind detonation propagation can be understood by considering triple-point trajectories over time (Figure 2.9). This schematic shows the shock waves, their direction of travel, and the reaction zone thickness. Interactions between triple points, which also correspond to the head-on collision of adjacent transverse shock waves, generate very high local temperatures and pressures producing a local explosion. These collisions govern a pattern of detonation initiation, decay and re-initiation, and the velocity and strength of the lead shock follows this pattern accordingly. This series of processes occurs over the length of one detonation cell, labelled $\mathrm{L}_{\mathrm{c}}$ in Figure 2.9.

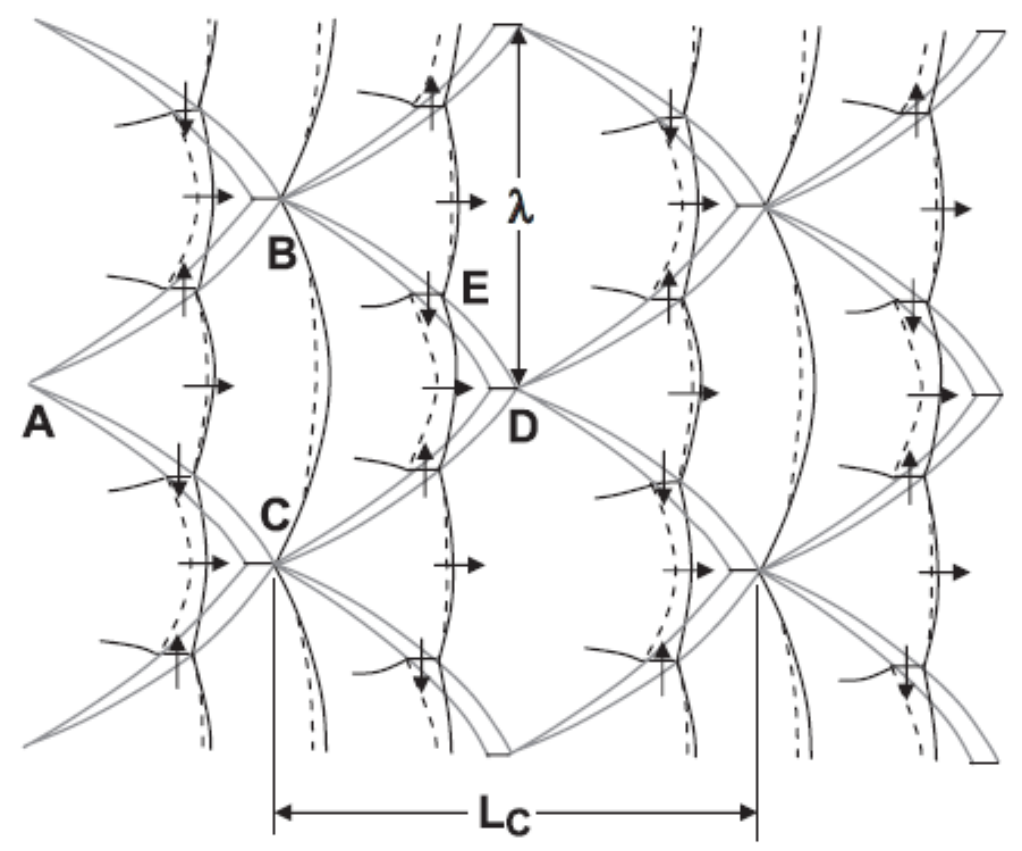

Figure 2.9: Schematic showing an ideal detonation front propagating left to right and its triple point trajectories. Solid dark lines represent shock waves, dotted lines represent the end of the induction zone where recombination reactions start. $L_{c}$ shows the characteristic cell length and $\lambda$ indicates detonation cell size [25].

Consider the formation of a single detonation cell, labelled at its vertices by Points A, B, C and D. The start of the detonation cell, Point A, is formed by the collision of two transverse waves (not shown in diagram), which reflect and propagate away from each other. This collision generates a strong lead shock, the Mach stem, which is coupled to the very short reaction zone (essentially a curved detonation wave). 
Initially, this detonation wave travels at approximately 1.6 times the CJ detonation velocity, but weakens over time as the reaction zone grows thicker (the induction length increases with decreasing post-shock temperature). Points B and C mark collisions of the transverse shocks with the transverse shocks in the adjacent cells. At these points, the transverse shocks reflect and begin propagating towards each other and Point D.

Over the second half of the cell (Points B and C to D), the lead shock becomes the incident shock. The incident shock continues to weaken, slowing to approximately 0.6 times the CJ detonation velocity. At this point, the reaction zone has decoupled from the lead shock, and locally the detonation has become a shock followed by a deflagration. The end of the detonation cell, Point $\mathrm{D}$, is formed by the collision of two transverse waves, which reinitiates the detonation. A strong Mach stem is generated at Point D and a new detonation cell begins to form.

\subsubsection{Soot Foils to Understand Detonation Propagation}

Experimentally, triple-point trajectories (such as those shown in Figure 2.9) are inscribed on soot foils, leaving a distinctive 'fish-scale' pattern of cells. Soot foils are thin pieces of material (typically metal) which are lightly coated with soot and installed inside a combustion chamber with the sooted-side facing inward so that it is exposed to the combustion. The detonation cell width (also colloquially known as size), $\lambda$, can be measured from soot foils and used to characterize the reactivity of a given mixture. Detonation cell size is inversely proportional to a mixture's reactivity and the regularity of cell size is also characteristic of the mixture. Stable mixtures, such as hydrogen-oxygen diluted with argon, have very regular cellular patterns displaying uniform cell size across the foil, while unstable mixtures have greater variation in cell size and irregular cellular structure. Stoichiometric hydrogen-oxygen is classified as an unstable mixture [26]. A soot foil showing the detonation cell size is shown in Figure 2.10. 


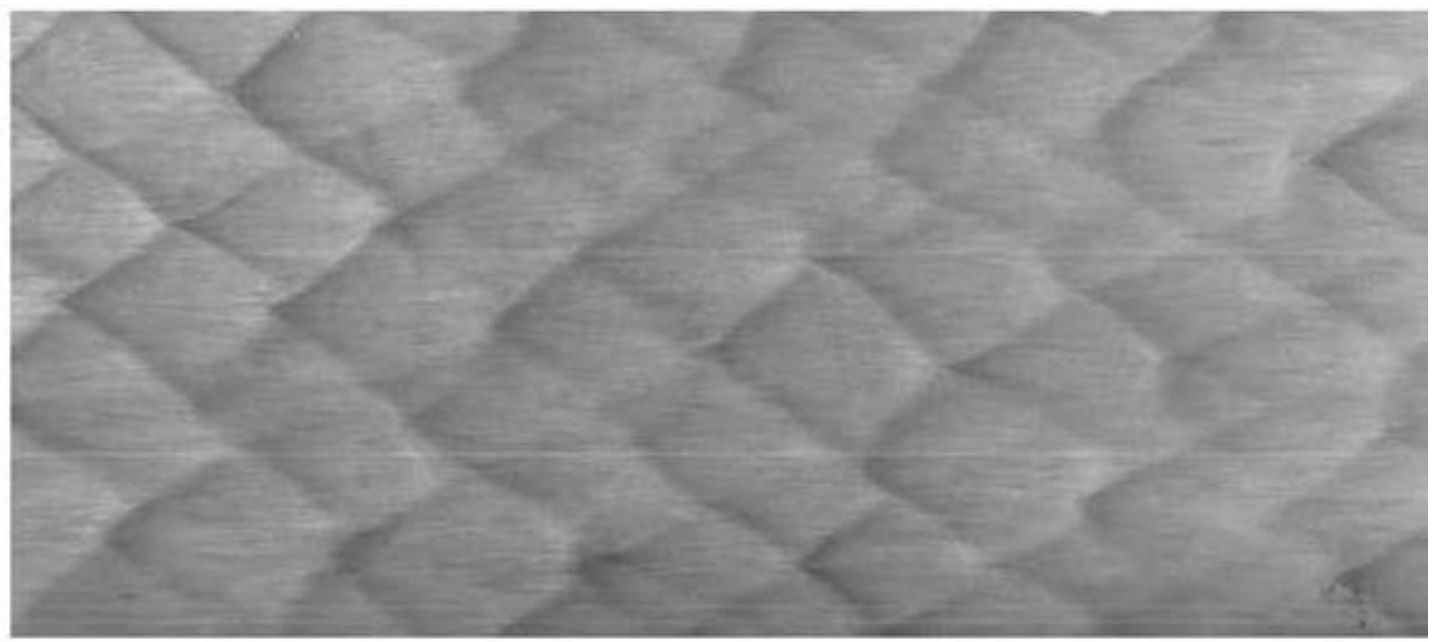

\section{Figure 2.10: Soot foil obtained for a hydrogen-oxygen mixture diluted with argon showing regular detonation cells [27]}

Soot foils are instrumental tools in understanding detonation propagation and helped explain an interesting propagation phenomenon known as 'single-head spin'. Detonations near the detonability limit (the limit below which less reactive mixtures will not detonate) propagate via the 'single-head spin' mechanism in smooth, round tubes. This propagation mode is characterized by a detonation front (also known as a detonation head) moving in a helical pattern down the tube. Although 'single-head spin' was first observed in 1926 by Campbell and Woodhead [28], based on the unique pattern that developed on their soot foils, Denisov and Troshin [23] realised that single-head spin is not governed by a different mechanism than the other detonation modes they had observed. Instead, in the case of single-head spin there is a single triple point propagating down the tube, unlike in multi-head detonations where two or more triple points collide and propagate down the tube.

However, soot foils have their limitations as demonstrated in the study of another interesting, nearlimit phenomenon: 'galloping detonations'. This propagation mode is characterized by choked flame propagation with intermittent detonation initiation and subsequent failure. In long, smooth tubes, the spacing between detonation re-initiations is often on the order of hundreds of tube diameters, making the galloping phenomenon difficult to observe in shorter tubes and very challenging to study due to the size of 
soot foils required. Galloping detonations are primarily observed in unstable mixtures. To effectively study galloping detonations, visual techniques or high-temporal resolution velocity measurements are required, such as Lee et al.'s [29] study of galloping detonations using laser Doppler interferometry to track velocity fluctuations within a rough-walled tube.

\subsubsection{Critical Tube Diameter}

The critical tube diameter, $\mathrm{d}_{\mathrm{c}}$, is the minimum tube diameter from which a detonation wave can be transmitted into an unconfined space filled with the same gaseous mixture, without failing (transitioning to deflagration). Expansion waves are generated as the detonation wave exits the tube and diffracts around the corners into the unconfined space. These expansion waves weaken the lead shock, and ultimately the detonation, causing a reduction in the post-shock temperature and a lengthening of the reaction zone. If the reaction zone becomes sufficiently long, it will decouple from the lead shock and the detonation will become a deflagration. This transition will occur at the outer portions of the detonation wave first, where it diffracts the full $90^{\circ}$. The diffraction processes which make the detonation wave curve also reduces the post-shock temperature. The detonation along the channel centreline will fail last, as it undergoes the smallest diffraction. If the tube diameter is less than the critical condition, the expansion waves reach the centreline and the reaction zone and shock will fully decouple even at the centreline. At this point, the detonation has failed. This scenario is shown in an open shutter photograph in Figure 2.11a, and is referred to as sub-critical. 

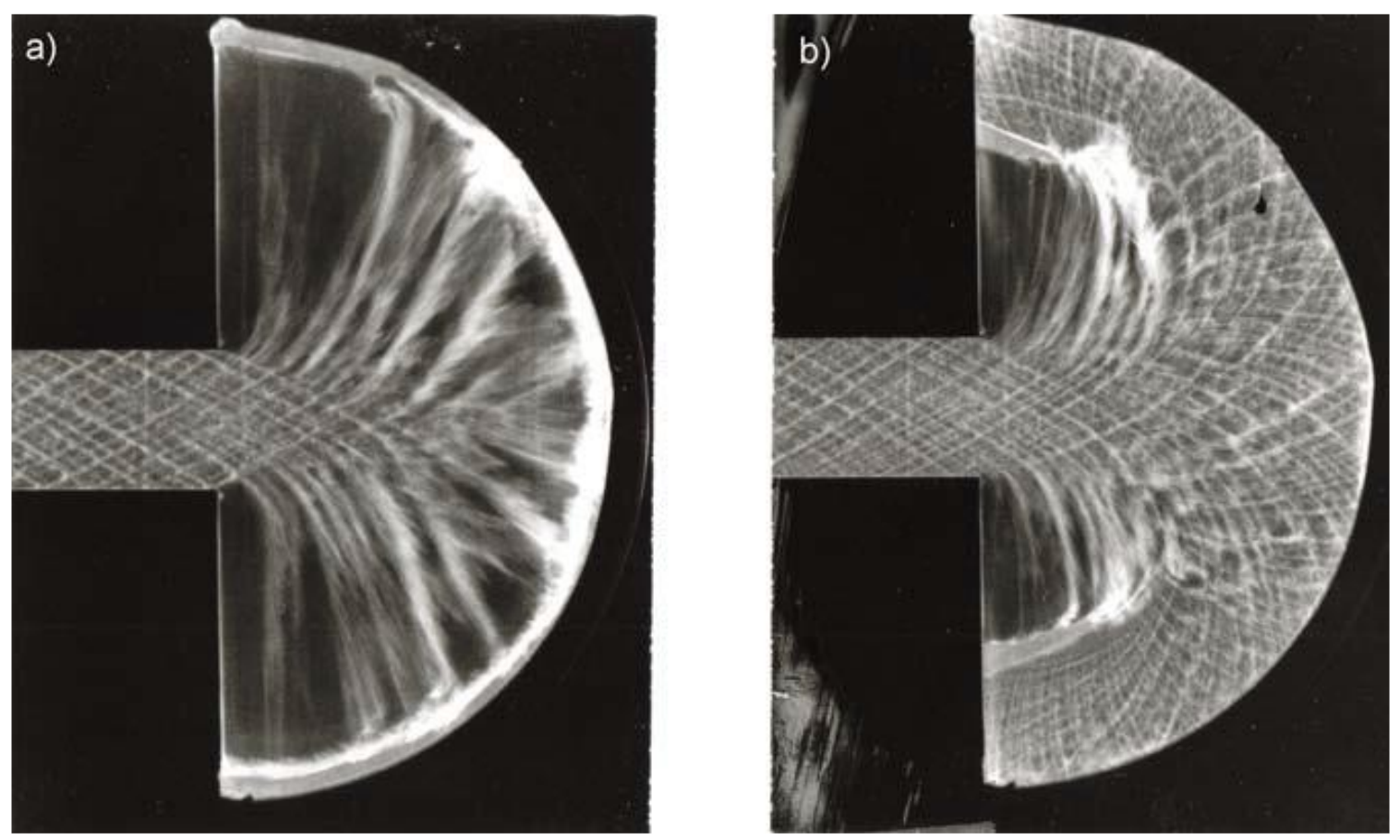

Figure 2.11: Open-shutter photographs of a detonation being transmitted from a channel into an unconfined space showing a) the sub-critical case where the detonation fails completely and b) the supercritical case where the detonation reinitiates as a cylindrical detonation [30].

For a test performed in a tube diameter much larger than the critical diameter, the detonation wave survives the diffraction process. This case, shown in Figure $2.11 \mathrm{~b}$, is referred to as super-critical. In this case, when the expansion waves reach the centreline, the resulting local detonation wave curvature is not significant enough to decouple the shock and reaction zone.

For a test performed with a mixture in a tube diameter equal to, or barely greater than, the critical tube diameter, the decoupling process starts, but a detonation is reinitiated behind the lead shock. Near the centreline, local overdriven explosions occur in the shock-heated region of unburned gas between the decoupled reaction zone and lead shock. The detonation sweeps along this region and reinitiates the detonation front. Figure 2.12 presents a sketch of the detonation failure and re-initiation mechanism at the critical condition. The local explosion centre grows in a detonation bubble which propagates between the reaction zone and shock. 


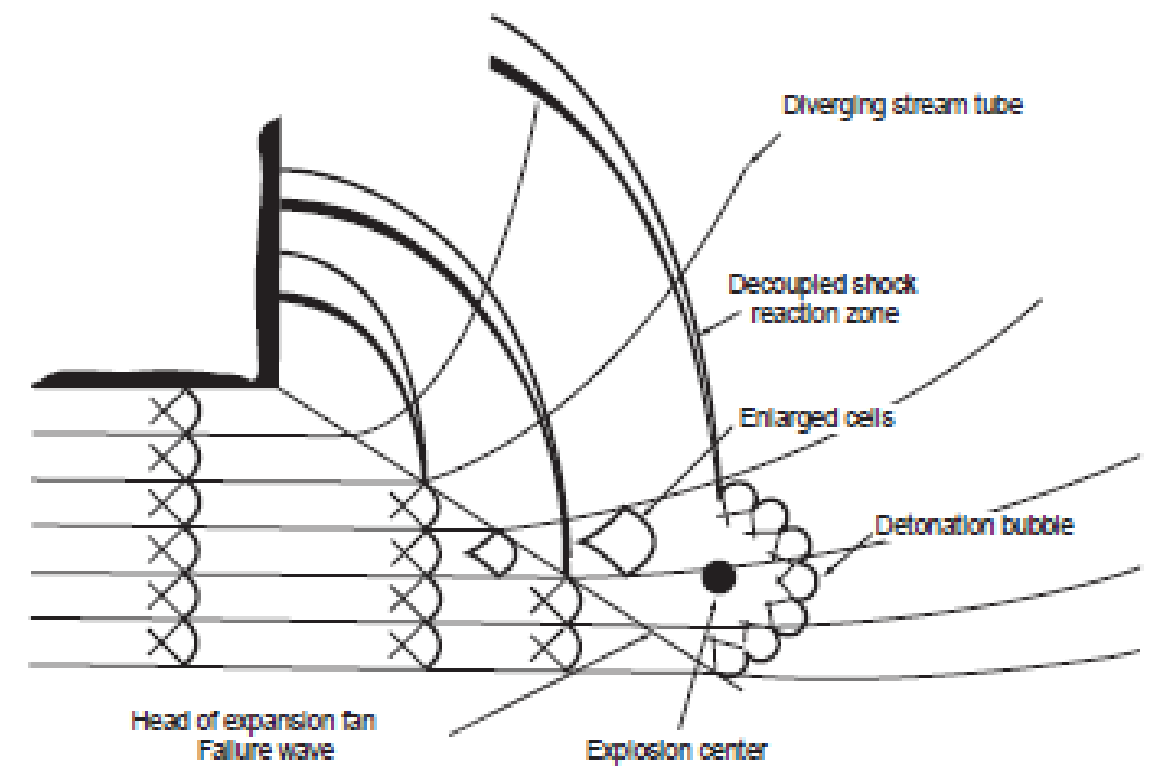

Figure 2.12: Sketch of failure and re-initiation mechanism of a detonation emerging from a tube into an unconfined space [30]

The critical tube concept was first explored in 1957 by Zeldovich [31] who determined the critical tube diameter was related to the width of the induction zone. In 1963, Schelkin and Troshin [32] proposed that the experimentally measured detonation cell size was proportional to the theoretical ZND induction zone length. This realization allowed Mitrofanov and Soloukhin [33] in 1965 to link the critical tube diameter with the detonation cell size. Mitrofanov and Soloukhin observed a correlation between cell size and critical tube diameter of approximately $d_{c}=13 \lambda$ for low pressure acetylene-oxygen detonations in round tubes. Knystautas et al. [34] verified this experimental observation and confirmed that this relationship holds for many common fuel-air mixtures. It was also shown experimentally for a detonation wave propagating in a round tube, the detonation propagation velocity approaches the $\mathrm{CJ}$ detonation velocity as the tube diameter approaches $13 \lambda$. Essentially, the critical tube diameter represents a condition where confinement boundary conditions have no effect on the detonation propagation. The correlation between critical tube diameter and detonation cell size is purely empirical, as there is no current theory to explain it. 
Similar detonation transmission studies were performed in rectangular channels. Instead of the critical tube diameter, the parameter used was the critical channel width $\left(\mathrm{w}_{\mathrm{c}}\right)$ corresponding to the narrow dimension of the channel. Mitrofanov and Soloukhin [33] and Edwards and al. [35] observed that the critical channel width corresponded to $10 \lambda$, compared to $d_{c} \approx 13 \lambda$ in round channels, and claimed this was in inline with the less severe 2D diffraction.

In 1984, Liu et al. [36] demonstrated the equivalence between an experiment in which a detonation is transmitted into unconfined space through a round orifice plate at the end of a smooth tube, and one in which the detonation is transmitted from a smooth tube with a diameter equal to the orifice diameter. They also performed experiments with different shaped orifices and found that for a rectangular opening the critical condition changed with the opening aspect ratio. Specifically, for a rectangular opening with a small aspect ratio the critical condition was found to be $d_{H}=13 \lambda$, where $d_{H}$ is the hydraulic diameter; whereas, for a rectangular opening with large aspect ratio the critical condition was found to be $w_{c}=3 \lambda$. This finding was at odds with the findings of Mitrofanov and Soloukhin [33] and Edwards and al. [35].

To address this discrepancy, Benedick et al. [37] in 1985 undertook a comprehensive study of the effect of a two-dimensional versus a three-dimensional geometry on critical channel width. They conducted large-scale experiments in a rectangular channel with a variable channel height, using hydrogen-air and acetylene air mixtures. By varying the aspect ratio of the channel, (where an aspect ratio approaching infinity represents a true two-dimensional case), they determined that for a two-dimensional geometry the critical channel width is approximately $3 \lambda$, corroborating the findings of Liu et al [36]. This correlation applied for aspect ratios (L/w) of approximately 5 to 34 , where $\mathrm{L} / \mathrm{w}=34$ was the largest aspect ratio tested. For a square channel (aspect ratio of 1 ), the $\mathrm{w}_{\mathrm{c}} \approx 10 \lambda$ criterion applies. The results of Benedick's experiments are summarized in Figure 2.13, a plot of critical channel height normalized to detonation cell size as a function of aspect ratio. 


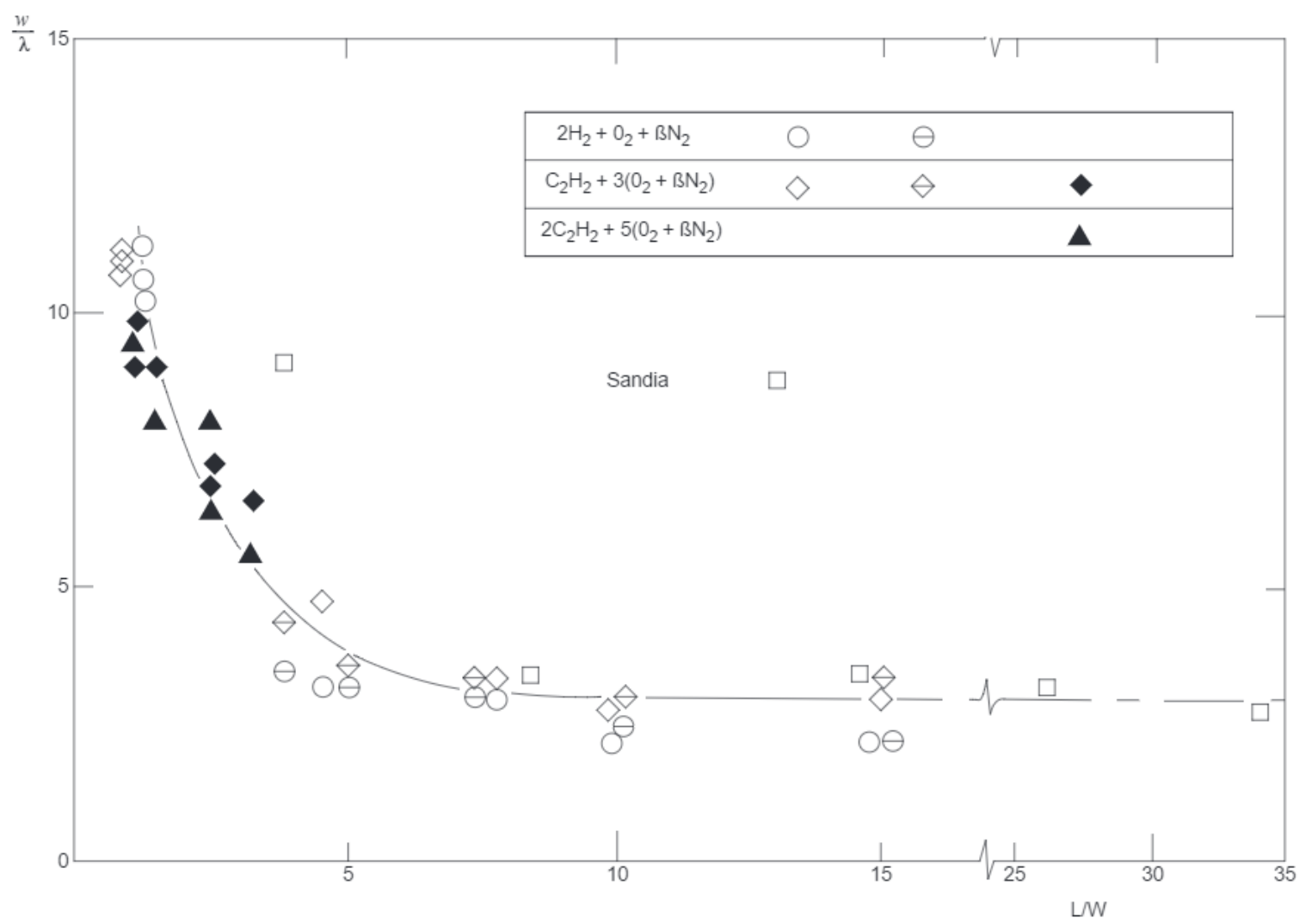

Figure 2.13: Plot of critical channel height normalized by detonation cell size as a function of channel aspect ratio [37]

\subsubsection{Detonation Initiation by Shock Reflection}

In classical critical tube diameter studies, such as the ones discussed in Section 2.1.5, the detonation wave is transmitted into unconfined space, meaning there are no walls downstream of the tube exit. However, if solid boundaries are present, the reflection of a shock wave generates high temperature and pressure in which local auto-ignition, and possibly DDT can occur [38]. For example, a shock which reflects off an obstacle, or end-wall, will propagate backwards into the region of pre-heated, unburned gas between the lead shock and trailing deflagration [39].

To study the role of shock reflection in the decoupling and recoupling of detonations, Pantow et al. [40] used Schlieren photography in a modified version of the classic critical channel width apparatus. Their 
apparatus consisted of two channels mated together where a detonation was formed in a smaller rectangular channel and then propagated into a larger rectangular channel. It should be noted that instead of critical channel width, they were finding critical channel height, $h_{c}$, and that the expansion in their apparatus only occurred in the vertical direction as the smaller and larger channels were of the same width. The detonation underwent 2-D diffraction, instead of 3-D diffraction in the case of a round tube, and shock reflection occurred on the top and bottom surfaces of the larger channel. Tests were completed using stoichiometric hydrogen-oxygen mixtures diluted with varying amounts of argon and nitrogen to study the effect on different mixture stabilities. Like the critical tube studies, there were three outcomes for detonation transmission depending on the critical channel height.

For a test with a channel height equal to or greater than the critical condition, a sequence of Schlieren images is presented in Figure 2.14. Note, Schlieren photography allows the visualization of density gradients associated with shock waves and reaction fronts. In the first image of the sequence, Figure $2.14 \mathrm{a}$, the detonation is seen emerging from the smaller channel and diffracting into the larger channel. The detonation decouples on the top and bottom sides due to diffraction, see Figure 2.14b. However, along the centerline the detonation does not fail, see Figure 2.14c. This is an example of the supercritical condition (channel height larger than the critical height), where the shock reflection on the top and bottom surfaces of the larger channel plays no role in the successful transmission of the detonation. 


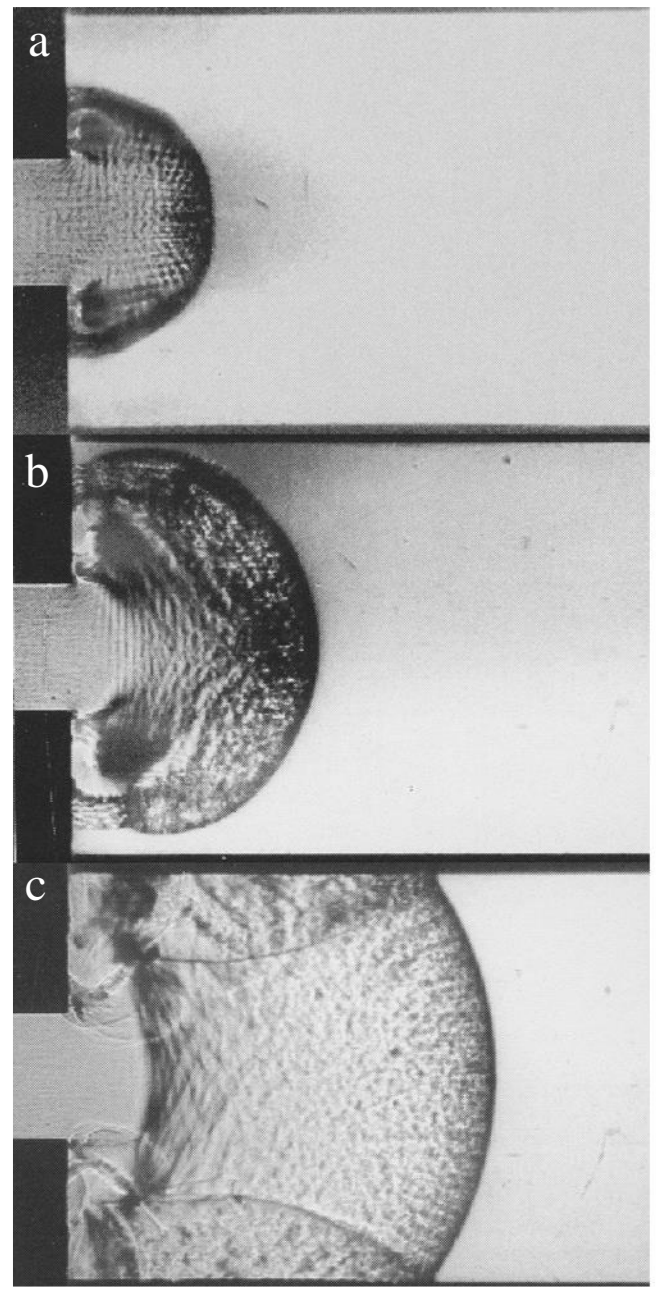

Figure 2.14: Critical or supercritical case where the detonation begins to fail, but is reinitiated by transverse waves [40]

A sequence of Schlieren images representing a subcritical channel height, is presented in Figure 2.15. In the first image of the sequence, Figure $2.15 \mathrm{a}$, a planar detonation is seen emerging from the smaller channel and beginning to fail, as indicated by the thickening of the reaction zone. By the second image, Figure 2.15b, the detonation is failing due to diffraction over the entire surface, including at the centerline, via the mechanism described in Section 2.1.5. In Figure 2.15c, the lead shock reflects off the top and bottom channel walls, generating a strong Mach stem that becomes a detonation wave. The detonation propagates into the region of heated, unburned gas between the shock and deflagration, as seen in Figure 2.15c. If the 
shock reflection off the channel walls did not occur, as in critical channel width tests, the detonation would have failed and not reinitiated.

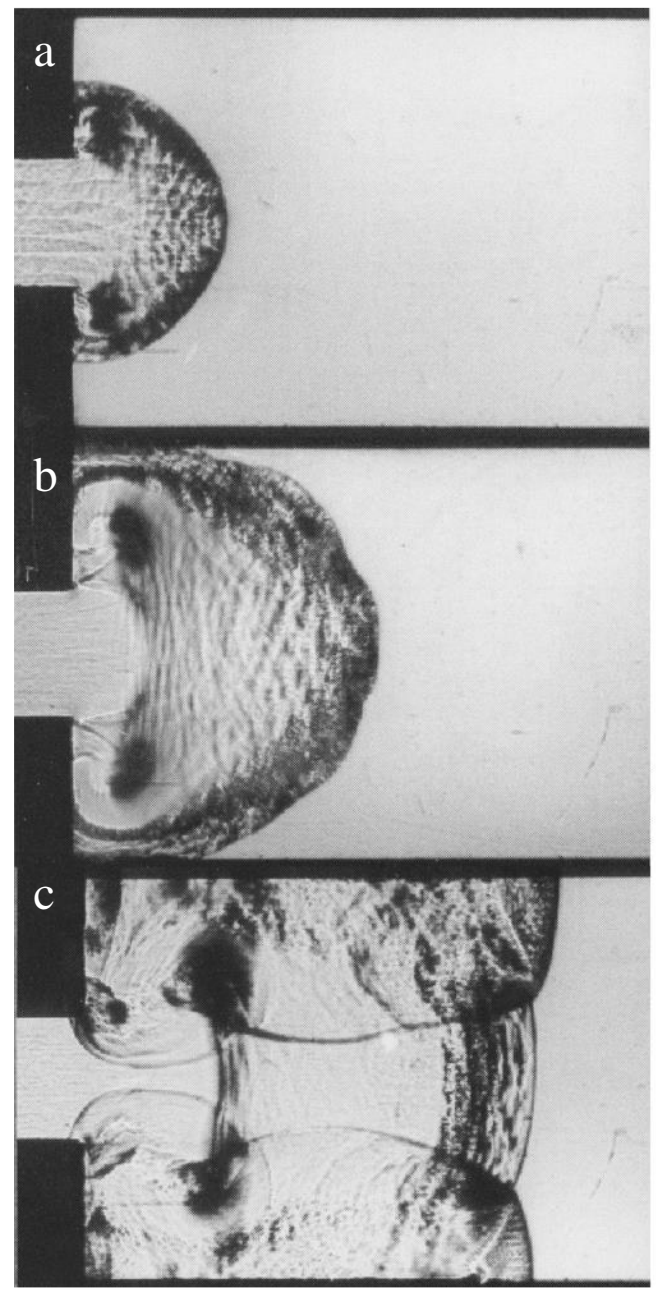

Figure 2.15: Schlieren photograph showing a planar detonation being transmitted into an expansion, subsequent failure of the detonation and then detonation re-initiation upon Mach reflection on the walls [40]

The final case, presented in Figure 2.16, shows a detonation that fails and does not reinitiate despite shock reflection off the channel walls. The detonation, shown emerging from the smaller channel in Figure 2.16a, is failing in the next image (Figure 2.16b) as seen by the lead shock wave and the trailing decoupled reaction zone across the entire front. The detonation has completely failed by the third image, Figure $2.16 \mathrm{c}$, where the thick reaction zone has fallen very far behind the lead shock. 


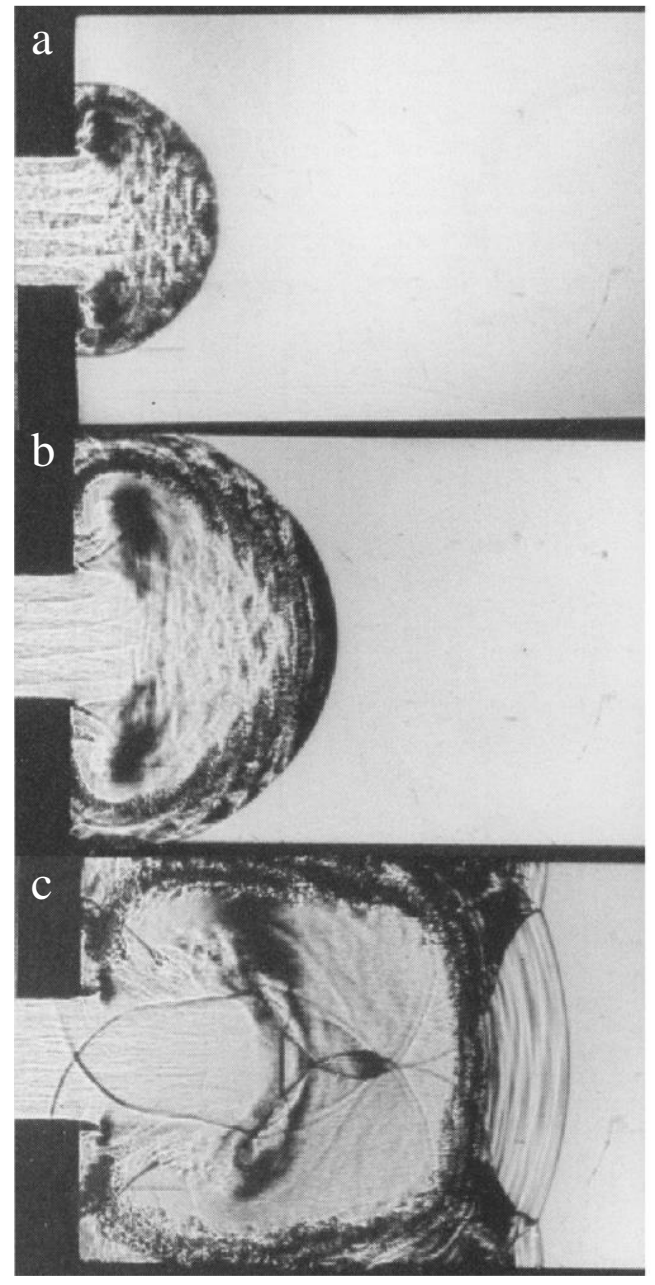

Figure 2.16: Sequence of Schlieren images showing a detonation emerging from a smaller channel and failing completely once through the expansion [40]

\subsection{Deflagration-to-Detonation Transition in a Channel Equipped with Orifice Plates}

Under certain conditions, a combustion wave may transition from the deflagration mode of combustion to the detonation mode. This change is known as a deflagration-to-detonation transition (DDT). However, once a deflagration has transitioned to a detonation, it is not locked into this mode, and can transition back to a deflagration. This occurs when the lead shock decouples from the combustion front and it is said that the detonation has failed. This section explains the flame acceleration process leading up to DDT and reviews previous DDT studies in a channel equipped with orifice plates. 


\subsubsection{Flame Acceleration in an Channel Equipped with Orifice Plates}

Prior to Chapman and Wheeler's 1927 study [3], flame acceleration was studied in long, smoothwalled tubes. Chapman and Wheeler discovered that they could generate much faster flame acceleration by installing repeating orifice plates in their tube, spaced one tube diameter apart. In their new experimental configuration, they observed flame speeds 40 times higher for the methane-air mixture compared to their previous experiments.

In 1940, Shchelkin [41] pioneered an alternative method of inducing flame acceleration. Dubbed the Shchelkin Spiral, Shchelkin installed a metal coil on the inside of the tube to enhance turbulence and thus flame acceleration. Although his spiral is still used today, Shchelkin's main contribution to the field was his proposed mechanism for flame acceleration. He realized that the turbulence in the unburned gas ahead of the flame caused an increase in the flame surface area and therefore volumetric burning velocity. In these early studies, non-optical flame diagnostic tools were used to determine the average combustion front velocity and study flame acceleration. In subsequent years, shadowgraph, Schlieren, PLIF and traditional high-speed photography has facilitated a better understanding of the flame acceleration process in the lead-up to DDT.

A schematic explaining the mechanism of flame acceleration in a channel equipped with repeating orifice plates is presented in Figure 2.17. The channel is closed at both ends and filled with a quiescent fueloxidizer mixture. A weak igniter initiates combustion in the fuel-oxidizer mixture at one end of the channel, generating a laminar flame which propagates down the channel (Figure 2.17a). In a phenomenon called 'flame-folding,' shown in Figure 2.17b, the introduction of large-scale turbulence increases the surface area of the flame which leads to an increased volumetric burning rate. The increased volumetric burning rate produces a larger velocity in the unburned gas ahead of the flame, which in turn increases the volumetric burning rate, completing the positive feedback loop that leads to flame acceleration. At the same time, a series of compression waves are generated as the flame pushes the unburned gas ahead of it. At approximately $300 \mathrm{~m} / \mathrm{s}$ (the speed of sound in the reactants), these compression waves merge to form a leading shock (shown in black). Flame-shock interactions introduce flame instabilities that further increase 
the volumetric burning rate. Once the deflagration accelerates to approximately half the CJ detonation velocity, it is referred to as a fast-flame (Figure 2.17c). Although, fast-flames travel supersonically relative to the stationary observer, they are still considered deflagrations because they have a subsonic velocity relative to the flow ahead of it. Furthermore, the combustion occurring is still governed by heat and mass diffusion. Eventually the fast-flame can transition to a detonation, shown in Figure $2.17 \mathrm{~d}$. If the conditions are conducive, the detonation can continue to propagate down the channel as a quasi-detonation, shown in Figure 2.17e.

a

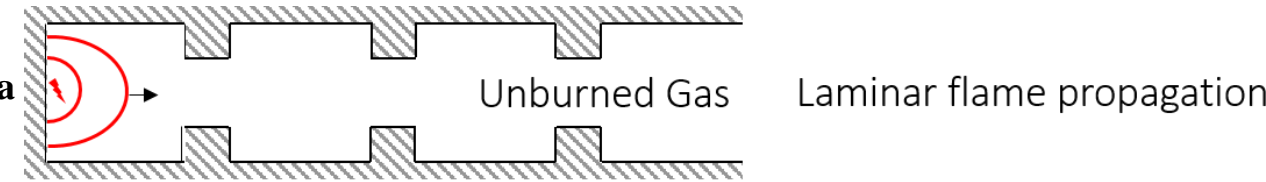

b

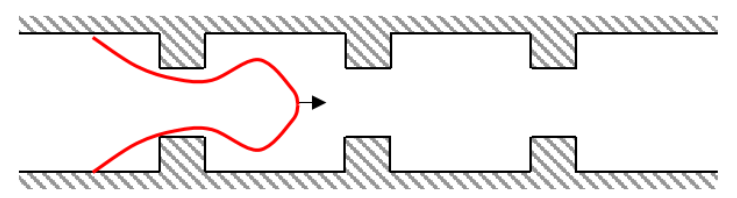

Flame folding

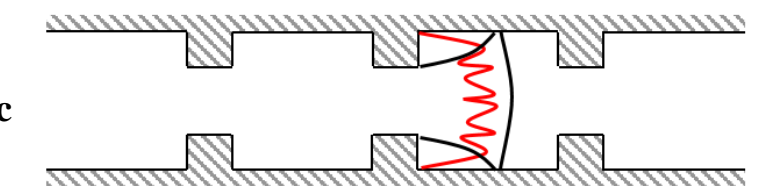

Fast Flame propagation

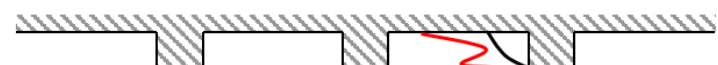

d

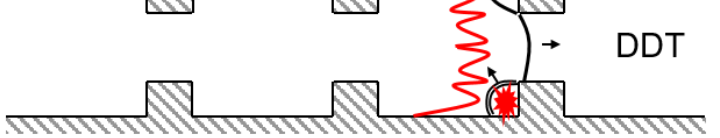

Deflagration-to-Detonation Transition

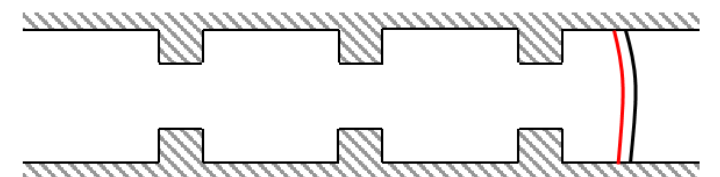

Quasi-Detonation propagation

Figure 2.17: Schematic showing typical flame acceleration in a channel equipped with repeating orifice plates [16]

A typical flame acceleration profile, such as the one shown in Figure 2.18, can be generated using flame-time-of-arrival measurements. The profile shows two cases: one where the laminar flame accelerates to become a fast-flame but never undergoes DDT, and one where DDT occurs. In the first case, where a 
fast-flame is produced, the velocity plateaus just below the isobaric speed of sound in the products. In the second case, where DDT occurs, the final combustion wave velocity falls between the theoretical CJ detonation velocity and the isobaric speed of sound in the products.

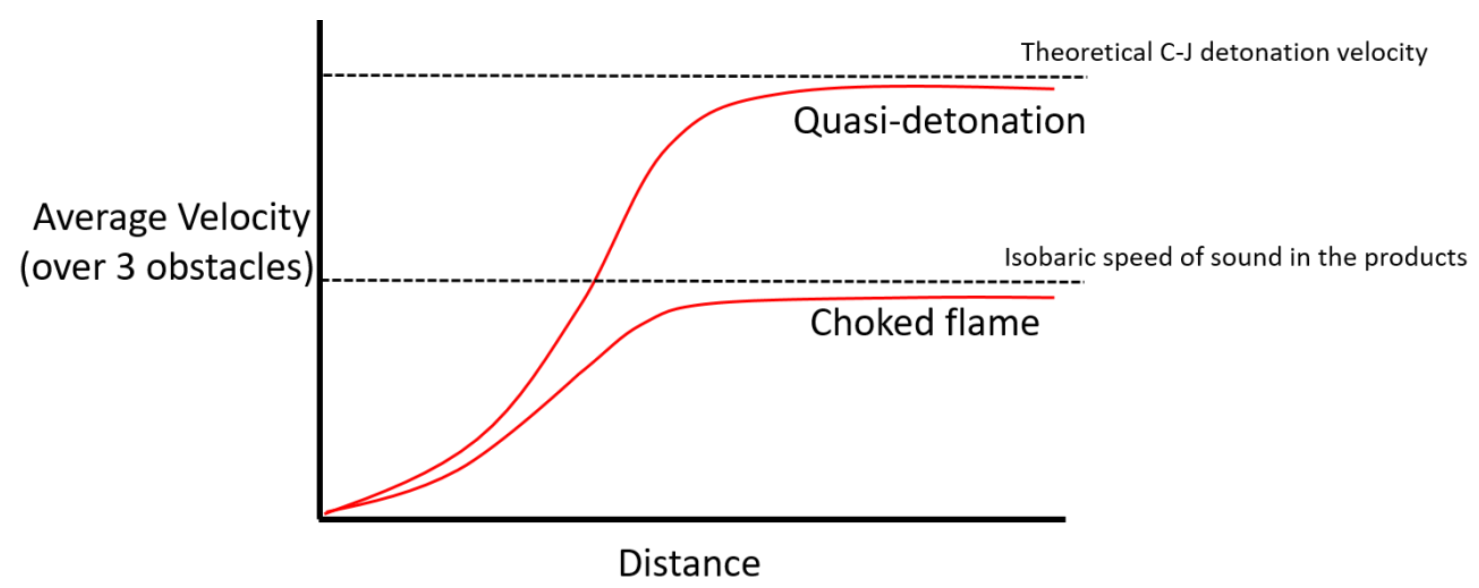

Figure 2.18: Typical flame acceleration profile for flow in an obstructed channel [16]

\subsubsection{DDT Limits}

Flame acceleration profiles such as those shown in Figure 2.18 can be used to determine DDT limits. The DDT limit is defined as the least reactive mixture in which DDT will occur. DDT limits are significant because DDT will not occur in mixtures that are less reactive than the limit. This is important from an explosion safety perspective in industrial pipe design, for example, due to the high overpressures associated with the DDT event as well as the resulting detonation.

DDT limits are typically determined by plotting the average quasi-steady-state velocity as a function of mixture reactivity. A mixture's reactivity can be varied by changing its chemical composition, resulting in a lean and rich limit. The reactivity can also be varied by changing the initial pressure, and therefore quantity of fuel, in the combustion chamber. DDT limits are both a property of the mixture and the combustion channel in which the tests are being completed. Currently, these limits are found 
experimentally, but ongoing research is being completed to develop numerical models using Computational Fluid Dynamics (CFD) and chemical kinetics to model when DDT will occur for a given mixture.

A plot of the average velocity measured at the end of a $10 \mathrm{~cm}$ diameter tube filled with repeating $7.5 \mathrm{~cm}$ diameter orifice plates for hydrogen-air as a function of hydrogen mole fraction is shown in Figure 2.19. Peraldi et al. [42] proposed three quasi-steady combustion wave propagation regimes: slow subsonic flames, fast supersonic flames and quasi-detonations. Low-speed turbulent deflagrations travel below the speed of sound in the reactants (approximately $300 \mathrm{~m} / \mathrm{s}$ ). High-speed turbulent deflagrations propagate between the speed of sound in the reactants and the products (approximately $1000 \mathrm{~m} / \mathrm{s}$ ). Quasi-detonations propagate at velocities between the CJ detonation velocity and the speed of sound in the products. The velocity deficit relative to the $\mathrm{CJ}$ detonation velocity depends on the mixture reactivity, diameter of the tube and the orifice blockage ratio. The blockage ratio (BR) is the ratio of the channel cross-section obstructed by obstacles to the total channel cross-section. A wave that propagates at the CJ detonation velocity implies that it is unaffected by boundary conditions, which is possible if the critical tube criteria is met. The most reactive mixture has a stoichiometric composition, which for hydrogen-air corresponds to a $30 \%$ hydrogen mole-fraction. The left-most and right-most white dashed lines in Figure 2.19 represent the lean and rich DDT limits, respectively. 


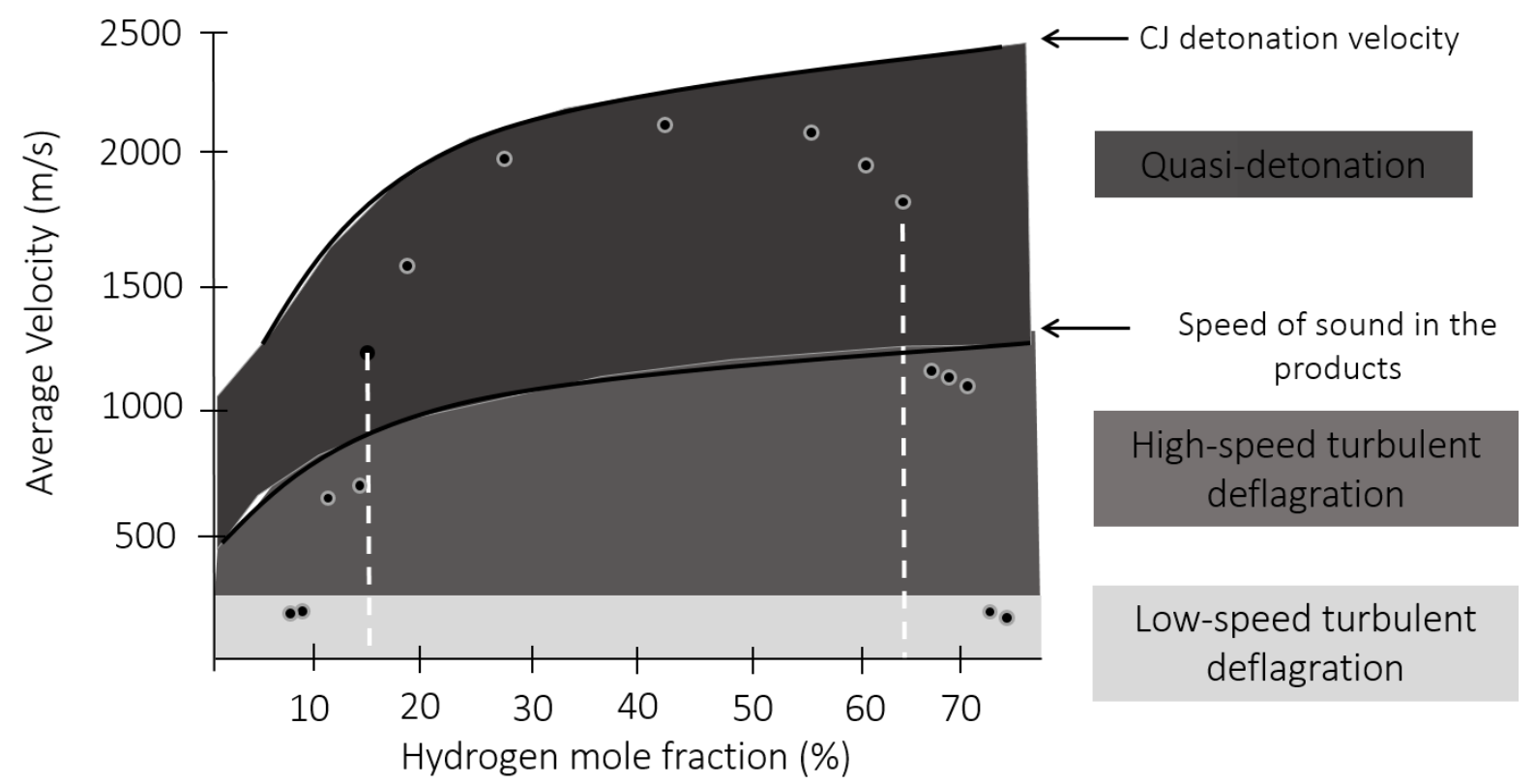

Figure 2.19: Plot of DDT limits for hydrogen-air as a function of hydrogen mole fraction [43]

Although the detonation limits are typically defined by a detonation velocity exceeding the speed of sound in the products, there is some ambiguity in this definition. In his overview of detonation phenomenon, Lee [30] discusses the difficulty determining limits for unstable detonations particularly given the challenge posed by galloping detonations. Galloping detonations travel at an overall average combustion front velocity of a fast flame (below the speed of sound in the products) but DDT still occurs, albeit infrequently.

\subsubsection{DDT Studies in Obstructed Channels}

In 1986, Peraldi et al. [42] performed the first comprehensive study of DDT in round tubes equipped with orifice plates. They completed tests using a broad range of fuel-air mixtures under Standard Atmospheric Temperature and Pressure (SATP) in three $18 \mathrm{~m}$ long tubes with a 5, 10 and $15 \mathrm{~cm}$ innerdiameter. The orifice plates used had a $43 \%$ blockage ratio and, as in Chapman and Wheeler's experiments, the orifice plates were spaced one tube diameter apart. The obstacle spacing and blockage ratio were 
selected to generate maximum flame acceleration and final propagation velocity. The average combustion front propagation velocity was determined using time-of-arrival measurements from equally spaced ion probes installed along the tube.

Based on their experiments, they proposed a simple DDT criterion; the orifice diameter, $d$, must be greater than the detonation cell size (i.e. $d / \lambda \geq 1$ ) for a detonation to form following flame acceleration. They observed that in a round tube with orifice plates, a quasi-detonation can sustainably propagate with a velocity deficit as high as $40 \%$ below the CJ detonation velocity. Experimentally, they demonstrated that the propagation velocity asymptotically approaches the CJ detonation velocity as $d \rightarrow 13 \lambda$, the same empirical relationship developed from the critical tube diameter experiments.

Kuznetsov et al. [11] studied the effect of obstacle blockage ratio on DDT in various diameter round tubes equipped with repeating orifice plates of different blockage ratios. They tested a range of blockage ratios from 0.09 to 0.9 and observed that DDT was more likely to occur in tubes with smaller blockage ratios. The likelihood of DDT occurring was apparent in the critical $\mathrm{d} / \lambda$ ratio they observed, which varied from 0.8 to 5.1 for $\mathrm{BR}=0.3$ to $\mathrm{BR}=0.6$. They also studied the effect of obstacle spacing on DDT and determined that increasing the obstacle spacing increased the likelihood of DDT occurring.

Ciccarelli and Cross [6] conducted a series of experiments in a $10 \mathrm{~cm}$ diameter round tube using hydrogen-air mixtures at SATP. A CJ detonation was generated in the unobstructed first half of the $6.1 \mathrm{~m}$ long channel and transmitted into the second half, which was filled with repeating orifice plates to generate a quasi-detonation. The inner diameter of these orifice plates was varied from $38.1-76.2 \mathrm{~mm}$ to study the effect of blockage ratio. Flat soot foils were installed along the channel centreline between orifice plates and curved foils along the tube wall to study the quasi-detonation propagation mechanisms.

The curved soot foils produced interesting cellular patterns never before observed, a sample is shown in Figure 2.20. Based on these foils it was proposed that for orifice diameters smaller than the critical tube diameter $(\mathrm{d}<13 \lambda)$, the detonation failed due to diffraction through the orifice plate and re-initiated at 'hot-spots' on the tube wall that formed following reflection of the decoupled leading shock. These hot- 
spots are recognizable on wall-soot foils as 'peaks' (marked by arrows in Figure 2.20) from which a triangular pattern of detonation cells originates and spreads. An overdriven detonation is initiated at the hot-spot that propagates circumferentially around the tube wall and forward in the direction of the shocked reactants. For very reactive mixtures, detonations were initiated at multiple locations along the tube wall and merged together as seen in Figure 2.20.

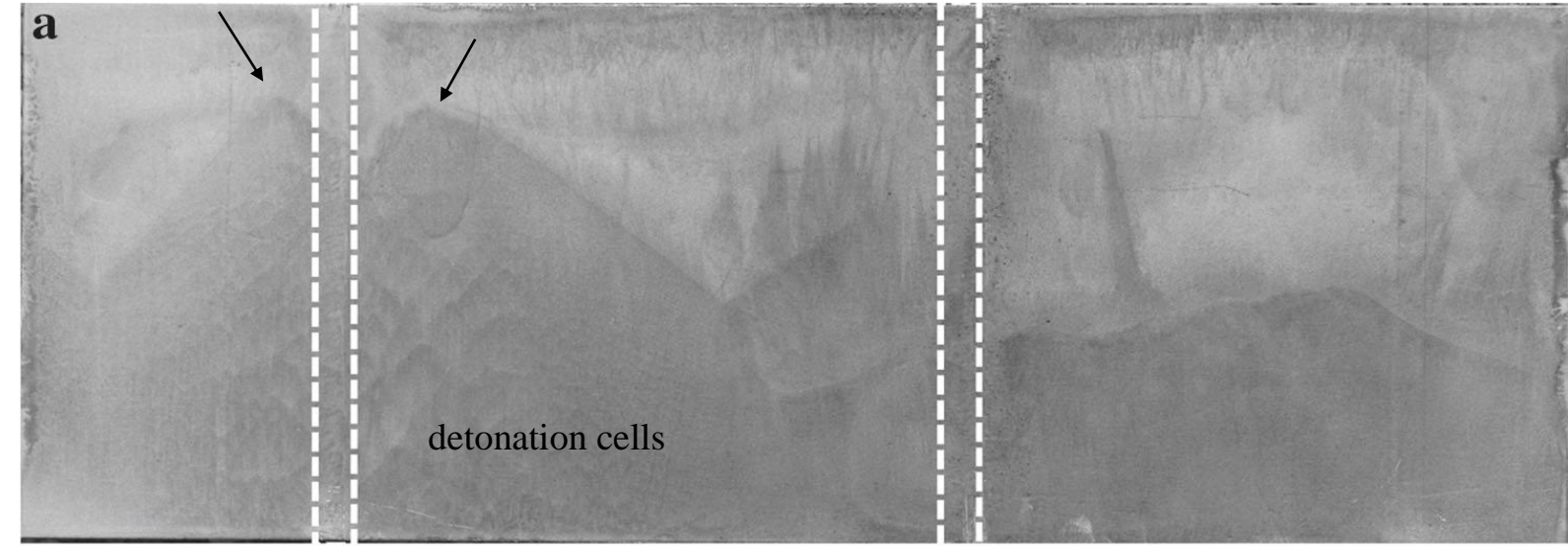

Figure 2.20: Soot foil from [26] a stoichiometric hydrogen-air test using a BR=0.44 showing two hot spots on the wall and emanating detonation cells. The vertical dashed lines indicate the position of rods used to secure the orifice plates.

For less reactive mixtures and mixtures near the limit, detonations were initiated at a single hotspot on the wall between orifice plates. The authors also noted that for mixtures very close to the propagation limit, the detonation did not need to reinitiate at a hot-spot on the wall between every set of obstacles, as established by the absence of detonation cells on some foils at tests at the limits. Ciccarelli and Cross did not observe single-head spin.

The variation in soot foils (and recorded cellular structure) located between adjacent orifice plates led Ciccarelli and Cross to conclude that even very reactive quasi-detonations are very transient in terms of 'detailed' detonation structure. Finally, they noted that the average quasi-detonation propagation velocity, determined by 'flame time-of-arrival' measurements was inversely proportional to the distance between the 
upstream orifice plate and the location where the detonation was re-initiated, that is, the closer the hot spots form after the orifice plate, the faster the average detonation velocity.

In 2001, Lieberman and Lee [4] conducted experiments using high-speed 'streak photography' to observe wave propagation of argon-diluted stoichiometric oxy-acetylene mixtures in a clear plastic tube equipped with repeating orifice plates. A rotating drum camera was used to capture the light from the narrow slit along the channel centreline. This technique provides detailed detonation front velocity data but the narrow slit provides very limited visual information at the channel centreline that can be used for better understanding of the detonation propagation mechanism. Experiments were conducted in a $4 \mathrm{~m}$ long, 50.8 $\mathrm{mm}$ diameter round tube using two sets of orifice plates corresponding to a $45 \% \mathrm{BR}$ and $55 \% \mathrm{BR}$. The distance between orifice plates was varied between $1 / 4$ tube diameter (D) to 1 tube diameter. The present research uses a 1D orifice plate spacing. In the quasi-detonation regime, Lieberman and Lee observed minor fluctuations in quasi-detonation velocity over between obstacle pairs but the velocity was approximately constant. Between a pair of obstacles, the streak photography recorded a velocity decrease as the wave propagated through the orifice plate followed by a sharp acceleration. This pattern of velocity decay and regeneration occurred over the range orifice plate spacings ( $1 / 4 \mathrm{D}$ to $1 \mathrm{D})$. In the fast-flame regime, Lieberman and Lee observed reoccurring bright spots between orifice plates which were coincident with or very slightly behind the leading reaction front, for a $1 \mathrm{D}$ orifice plate spacing. To study the effect of mixture regularity on detonation failure mechanisms, the argon dilution of the stoichiometric mixtures was varied, with highly diluted mixtures representing very regular mixtures. Two different failure mechanisms for regular and irregular mixtures were observed. In very regular (heavily Argon diluted) mixtures, detonations failed due to severe curvature of the detonation front. In irregular mixtures, detonation failure resulted from detonation diffraction past the obstacle causing cooling of the detonation cells.

Soot foils were instrumental in determining the 3D structure of a steady CJ detonation wave [23]; however, they only show the triple-point trajectories along the wall foil and do not provide any information on the shock and reaction fronts. The invention of high-speed CMOS cameras and an interest in better 
understanding detonation structure and propagation has led to the increased use of rectangular channels with fence-type obstacles in DDT studies. Rectangular channels with flat glass windows allow for the use of Schlieren photography to visualize the shock waves and reaction fronts. Because the Schlieren technique requires collimated light, it cannot be used in round tubes due to their curvature and resulting light distortion. Teodorczyk et al. [7], and Kellenberger and Ciccarelli [9] completed studies using high-speed, high-resolution Schlieren photography to visualize the structure and propagation mechanism of a quasidetonation wave. Boeck et al. [10] used simultaneous Schlieren and PLIF photography, and recently Kellenberger et al. [9] used Schlieren photography filmed through transparent glass soot foils to gain a better understanding of detonation propagation.

Given the challenge posed by galloping detonations to defining detonation limits, the ability to observe the detonation phenomenon is extremely valuable when developing detonation limits. Galloping detonations would not be apparent using non-optical time-of-arrival velocity measurements due to the averaging of the velocity over a distance, but would be clear using optical techniques. 


\section{Chapter 3}

\section{Experiment}

\subsection{Overview}

In this study, the propagation mechanisms of quasi-detonations in a round tube equipped with repeating orifice plates was investigated. An optically clear cylindrical channel was designed to attach to an existing square cross-section combustion channel. Premixed stoichiometric hydrogen-oxygen mixtures at initial pressures between 4 and $60 \mathrm{kPa}$ were loaded into the combustion channel and ignited at one end. Equally spaced fence-type obstacles in the square channel and orifice plates in the round tube were used to promote flame acceleration. Two sets of orifice plates were used to study the effect of blockage ratio, BR, versus obstacle opening size, $d$, in the round and square channels. The $50 \%$ BR orifice plates maintained the same blockage ratio as the square channel, while the $75 \%$ BR orifice plates had the same obstacle opening size as the square channel. The average combustion front velocity in the round tube was measured by tracking the combustion front using high-speed video. In the square channel, this velocity was obtained using the shock time-of-arrival at two fast-response piezoelectric pressure transducers. Two synchronized high-speed cameras were used to observe detonation propagation in the round tube, and in select experiments soot foils were installed in the end of the cylindrical channel.

\subsection{Combustion Channel}

The combustion channel consists of three sections: a $3.16 \mathrm{~m}$ long aluminum square channel, a 1.55 m long acrylic cylindrical channel and a square cross-section dump tank. For some low pressure tests the dump tank was removed and replaced with an optically accessible end flange. A Solid Edge model of the combustion channel with the optical end flange is presented in Figure 3.1. The cylindrical combustion channel was designed to mount to the existing square combustion channel via a transition flange.

Initial tests were completed with five segments of the square channel (including one optical section) and a single cylindrical channel of length $69.2 \mathrm{~cm}$. However, to ensure steady-state a second cylindrical 
segment was installed. To allow for the extension of the cylindrical channel given the space constraints, one non-optical segment of the square channel was removed. Previous tests indicate that a four and five segment square channel are equivalent, except for tests performed at $10 \mathrm{kPa}$ where DDT occurs in the fivesegment channel but does not occur in the four-segment channel (a fast-flame enters the cylindrical section).

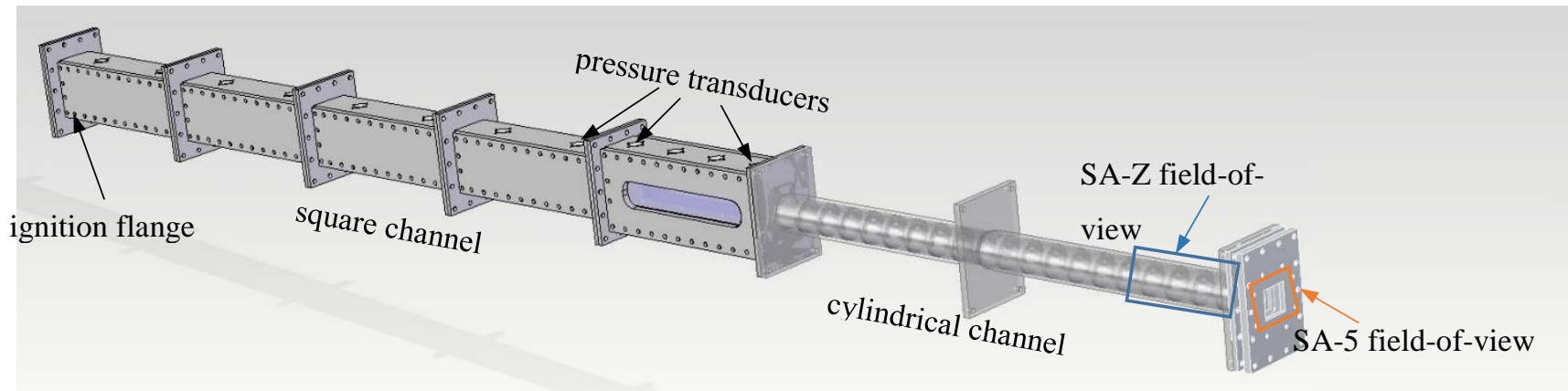

Figure 3.1: Solid Edge model of the combustion channel with the optical end plate installed

\subsubsection{Square Channel}

The square channel consists of four $61.0 \mathrm{~cm}$ long modular sections and one $63.5 \mathrm{~cm}$ long optical section, with a continuous $7.62 \mathrm{~cm}$ by $7.62 \mathrm{~cm}$ square interior cross-section. It was designed by a previous student [44] and machined in-house from 6061-T6 Aluminum. The square segments were bolted together at the end-flange and sealed using BUNA o-rings. A model showing a cut-away of the interior is shown in Figure 3.2.

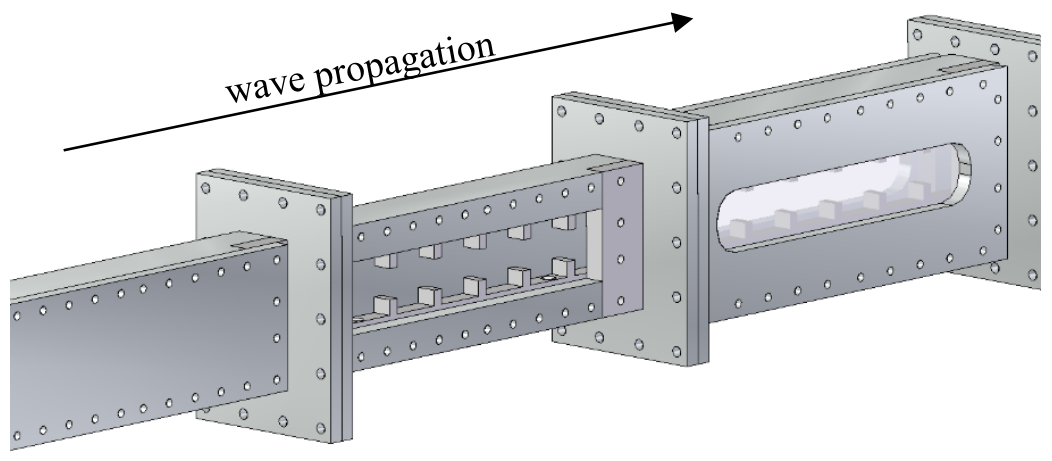

Figure 3.2: Solid Edge model of the square channel showing the interior cross-section 
To promote flame acceleration and DDT, aluminum 'fence-type' obstacles were installed on the top and bottom of the square channel, as shown in Figure 3.3. The $1.3 \mathrm{~cm}$ thick, $1.91 \mathrm{~cm}$ high and 7.62 $\mathrm{cm}$ long obstacles were spaced one obstacle length apart $(7.62 \mathrm{~cm})$ and formed a blockage ratio of $50 \%$. The blockage ratio is defined as

$$
B R=1-\frac{d}{H}
$$

where $\mathrm{d}$, is the opening between obstacles, and $\mathrm{H}$ is the channel height, as shown in Figure 3.3. There is a $3.81 \mathrm{~cm}$ opening, $\mathrm{d}$, between the fence type obstacles.

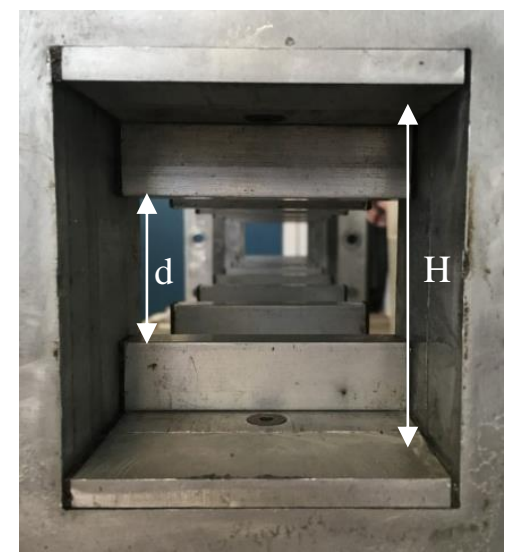

Figure 3.3: End-view down the square channel showing the opening, d, between the fence-type obstacles

\subsubsection{Cylindrical Channel}

The cylindrical channel was composed of two segments: a $69.2 \mathrm{~cm}$ acrylic tube and an $80.0 \mathrm{~cm}$ polycarbonate tube. The two tubes had a $10.16 \mathrm{~cm}$ outer diameter (OD) and an $8.89 \mathrm{~cm}$ inner diameter (ID). Both segments were optically clear and there was no specific reason for the difference in the materials. The field-of-view covering the last five orifice plates is located $1.09 \mathrm{~m}$ from the square-to-round channel transition, to allow the combustion front to stabilize and to eliminate transition effects resulting from the change in geometry. 
Three $1.91 \mathrm{~cm}$ thick acrylic flanges with a $7.62 \mathrm{~cm}$ diameter hole through the centre, shown in Figure 3.4, were manufactured in-house. One flange was mounted to the end of the square channel and another was mounted to the dump tank (or optical end-plate), sealing with an o-ring. To align the round tube, a $0.16 \mathrm{~cm}$ recess was machined around the hole. Built into this recess was an o-ring groove, which sealed on the face of the round tube via a BUNA o-ring. The two round tube segments were mated using a flange that had a recess and o-ring groove on either side. Four $1.27 \mathrm{~cm}$ diameter holes were drilled in the corners of each flange to line up with the existing holes on the square channel flange.

The cylindrical channel was held together in compression using four $1.27 \mathrm{~cm}$ diameter threaded rods. These rods passed through the holes drilled in the acrylic flanges and the holes in the last flange of the square channel and optical end plate, and were bolted tight. The two outer flanges were reinforced with angle iron to prevent bending. The middle flange did not require reinforcement because it was subjected to a net neutral force. The outer tube was reinforced with nine $12.7 \mathrm{~cm}$ diameter aluminum hose clamps placed around the obstacles so as not to interfere with the video footage.

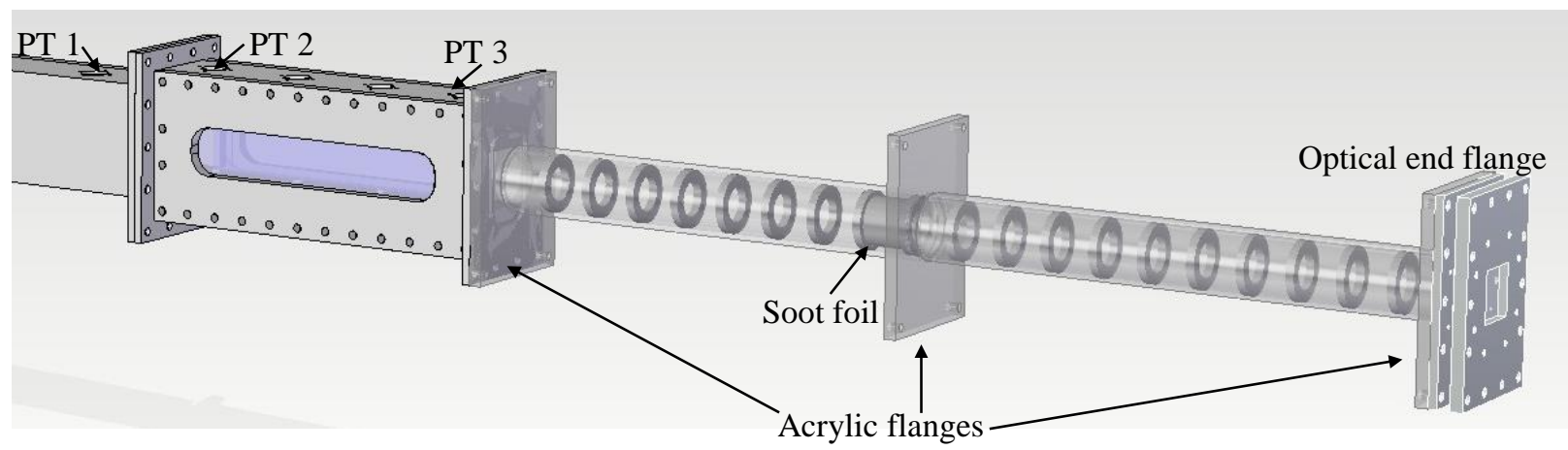

Figure 3.4: Solid Edge model of the cylindrical channel

Nineteen orifice plates were installed in the cylindrical channel, equally spaced, one tube diameter apart. Four full sets of orifice plates were machined in-house using the waterjet cutter. A set of optically clear acrylic orifice plates and a set of 6061-Aluminum plates were made for each blockage ratio. The 50\% blockage ratio orifice plates had an $8.9 \mathrm{~cm}$ outer diameter and a $5.33 \mathrm{~cm}$ inner diameter. The $75 \%$ blockage 
ratio plates had an $8.9 \mathrm{~cm}$ outer diameter and a $7.62 \mathrm{~cm}$ inner diameter. Figure 3.5 shows the acrylic and aluminum orifice plates for a $50 \%$ blockage ratio.
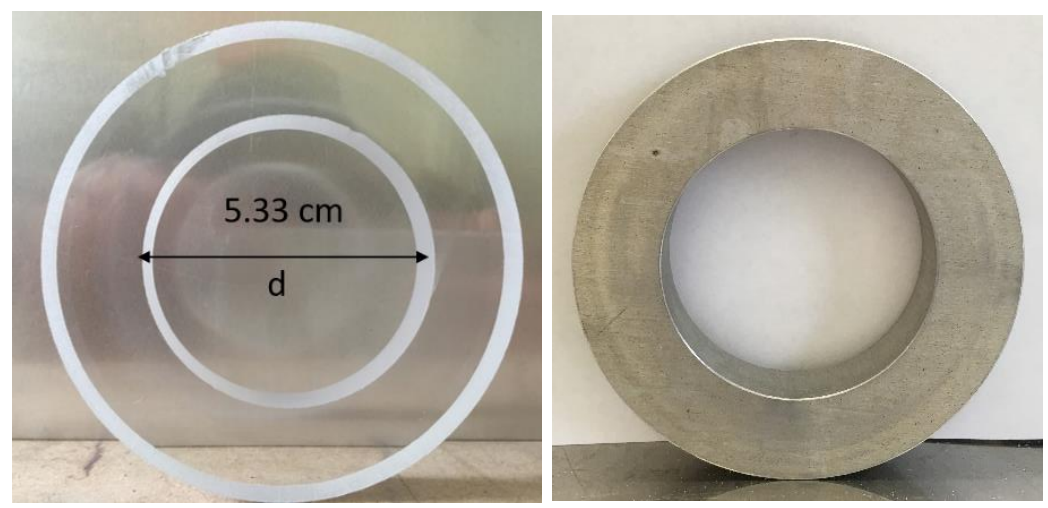

Figure 3.5: Acrylic (left) and aluminum (right) orifice plates for 50\% BR tests

The acrylic orifice plates were used with the optical end-flange to allow end-view video footage to be obtained. During the course of testing, side-view video indicated that tiny particles of plastic were being eroded from the inner diameter of the orifice plate and burned. Inspection of the orifice plates clearly showed burned regions around the inner diameter. Therefore for most of the testing, the aluminum orifice plates were used since they were not affected by erosion.

The separation distance between orifice plates was maintained by $6.4 \mathrm{~cm}$ long, $8.9 \mathrm{~cm} \mathrm{OD}, 7.6 \mathrm{~cm}$ ID optically clear acrylic spacers that fit tightly into the main tube. The spacer ends were machined square on a lathe and a lengthwise-slit was cut into the spacer to provide some flexibility to ensure they would fit inside the outer tube. The spacers also prevented any possible leakage of gas around the outside of the orifice plates. Periodically, these spacers needed to be cleaned. After many consecutive tests, the spacers became very cloudy and began obscuring the camera footage, so the cylindrical channel was disassembled and the spacers were polished using a shop cloth and Novus Fine Scratch Remover.

\subsubsection{Optical End Flange}

The optical end-flange was used to in conjunction with the acrylic orifice plates to allow video to be filmed down the length of the tube. Optical access was provided by a $7.62 \mathrm{~cm}$ by $7.62 \mathrm{~cm}$ acrylic window 
set into a rectangular aluminum end-flange. The optical end-flange, shown in Figure 3.6, was not used for tests with initial pressures greater than $25 \mathrm{kPa}$ out of concern that the high pressures generated by shock reflection on the end-wall would break the window. The end-flange was designed and manufactured by Kellenberger [45] for a previous thesis.

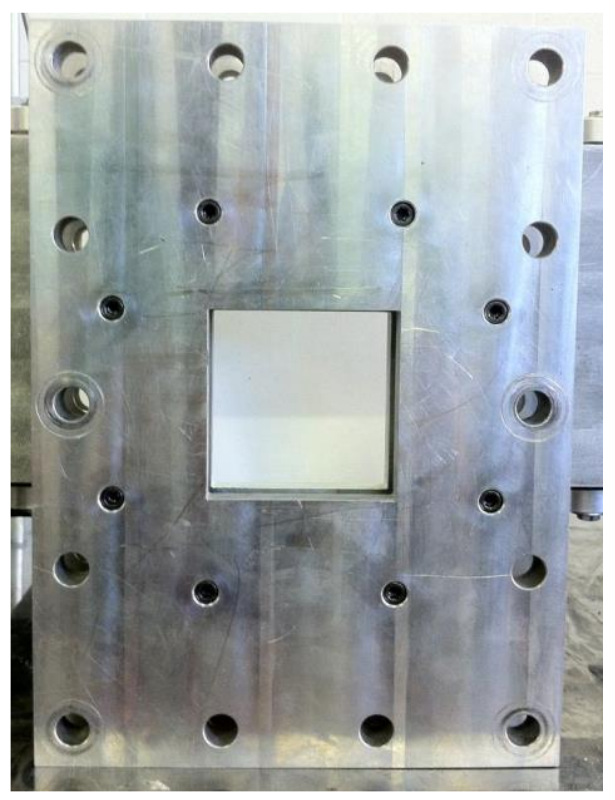

Figure 3.6: End flange providing optical access down the length of the tube [45]

\subsubsection{Dump Tank}

For most tests, an aluminum dump tank was used. The purpose of the $63.5 \mathrm{~cm}$ long, $12.7 \mathrm{~cm}$ by $17.15 \mathrm{~cm}$ cross-section channel, shown in Figure 3.7, was to locate the acrylic cylinder far from the endplate where high pressures were generated following shock reflection. 


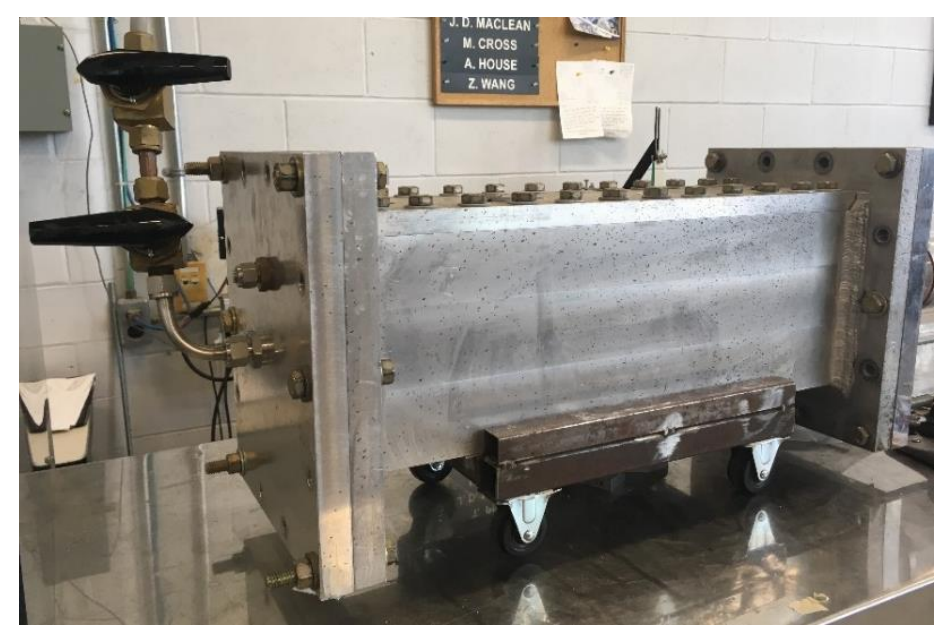

Figure 3.7: Photograph of the dump tank

\subsubsection{Mixing Chamber and Control Panel}

The stoichiometric hydrogen-oxygen mixtures were prepared in the mixing chamber using the partial pressure method in batches of $300 \mathrm{kPa}$. A new mixture was prepared once the pressure in the mixing chamber fell below atmospheric pressure in order to ensure no atmospheric air leaked into the chamber. An electric drill connected to an impeller was used to mix the gases for thirty minutes. The speed of the electric drill was controlled using a variable voltage meter. Figure 3.8 presents a schematic of the control panel and combustion channel connections. The control panel, used to operate the valves, is outlined in grey. 


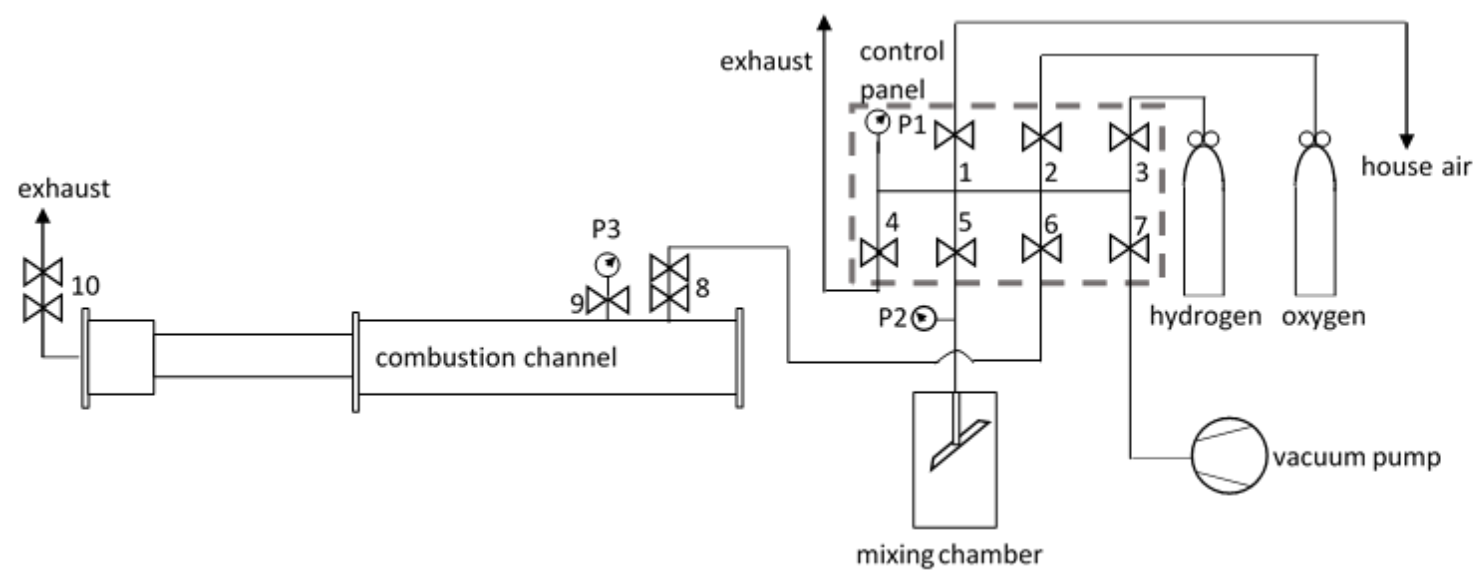

Figure 3.8: Schematic of control panel and valve connections

The functions of the numbered valves in Figure 3.8 are outlined in Table 3.1. There are two valves connecting the combustion channel to the control panel to protect the first valve against high pressures seen in the combustion channel. The operation of these valves and full operating procedure is outlined in Section 3.6.

Table 3.1: Valve Number and Function

\begin{tabular}{|c|l|}
\hline Valve Number & \multicolumn{1}{c|}{ Valve Function } \\
\hline 1 & House air to control panel \\
\hline 2 & Oxygen bottle to control panel \\
\hline 3 & Hydrogen bottle to control panel \\
\hline 4 & Exhaust to control panel \\
\hline 5 & Mixing chamber to control panel \\
\hline 6 & Combustion channel to control panel 1 \\
\hline 7 & Vacuum pump to control panel \\
\hline 8 & Combustion channel to control panel 2 \\
\hline 9 & Combustion channel pressure transducer \\
\hline 10 & Combustion channel exhaust \\
\hline
\end{tabular}

\subsection{Ignition and Data Acquisition (DAQ) Systems}

The data acquisition system used in these experiments consisted of an 8-channel National Instruments (NI) PCI-6133 Data Acquisition (DAQ) card connected to a NI BNC-2110 DAQ terminal 
controlled using NI SignalExpress ${ }^{\mathrm{TM}} 11$ software. SignalExpress ${ }^{\mathrm{TM}}$ software was also used to control the timing of the tests so that the DAQ and camera captured the combustion phenomenon immediately after ignition.

Three model 113A24 PCB piezoelectric pressure transducers were installed in the square channel, in the positions shown in Figure 3.1. The pressure transducers were mounted flush to the inner surface of the channel and were located $1.73 \mathrm{~m}, 1.99 \mathrm{~m}$, and $2.45 \mathrm{~m}$ from the ignition end of the channel. The exposed transducer ends were coated in silicone to protect against high temperatures and reduce noise in the signal. The pressure transducers have a voltage sensitivity of $5 \mathrm{mV} / \mathrm{psi}$ or $0.7252 \mathrm{mV} / \mathrm{kPa}$. Pressure data was collected at a sampling rate of $2.5 \mathrm{MHz}$ and the signal input range was set between $+5 \mathrm{~V}$ and $-5 \mathrm{~V}$.

The first pressure transducer was used to trigger the cameras and DAQ system for the glow plug ignition tests described in Section 3.3.2. The other two pressure transducers were used to calculate the combustion front velocity using shock-time-of-arrival measurements in the square channel.

\subsubsection{Shock-Time-of-Arrival Measurements}

The average combustion front velocity in the last square channel segment was calculated using shock-time-of-arrival measurements from the last two pressure transducers (PT 2 and PT 3, shown in Figure 3.4). A sharp pressure rise corresponds to the time that the lead shock passed the pressure transducer. Given the distance between pressure transducers $(30.5 \mathrm{~cm})$ and measured time interval, the average combustion front velocity, $\mathrm{v}_{\mathrm{c}}$, was calculated according to

$$
v_{c}=\frac{x_{P T 3}-x_{P T 2}}{t_{P T 3}-t_{P T 2}}
$$

The time-of-arrival was determined using pressure signal plots, such as the sample plot for a 30 kPa test given in Figure 3.9. 


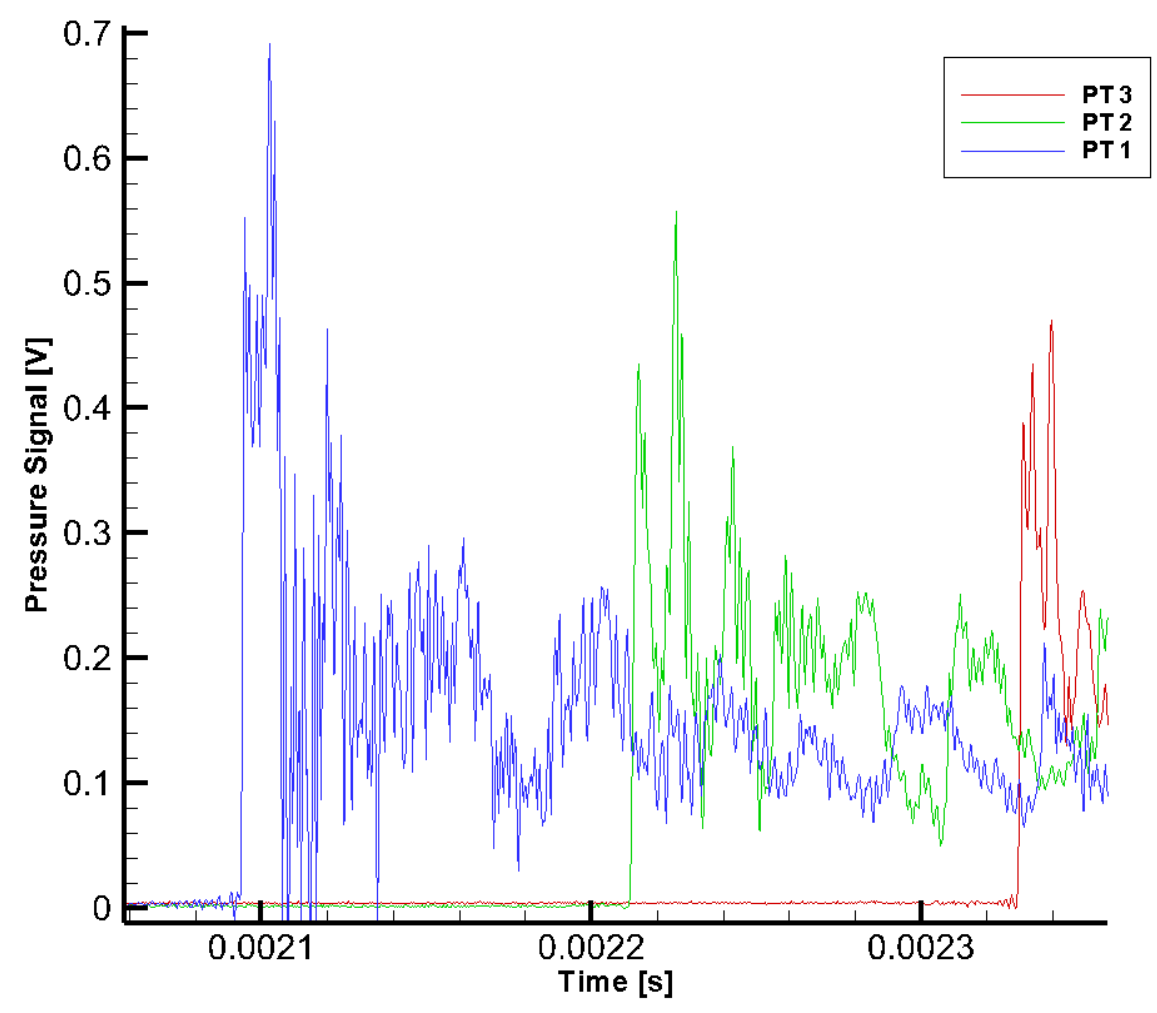

Figure 3.9: Plot of the pressure signals recorded as a function of time used for a $30 \mathrm{kPa}$ test (Test 161)

\subsubsection{Ignition Systems}

Most tests were completed using an automotive capacitive discharge system to ignite the mixtures. A spark plug connected to a high voltage power supply was mounted in the end wall of the flange and triggered using an NI SignalExpress ${ }^{\mathrm{TM}}$ program. When manually initiated, the NI SignalExpress ${ }^{\mathrm{TM}}$ outputted a $5 \mathrm{~V}$ TTL pulse to trigger the ignition system and cameras.

A second ignition system was developed for tests with initial pressures below $7.5 \mathrm{kPa}$, as the spark plug would not ignite mixtures at these pressures. The ignition end flange was modified to mount a Bosch automotive glow plug (model 0250 202127) into the channel below the spark plug, shown in Figure 3.10. The glow plug was mounted in a Swagelok fitting and was powered by a 12 VDC power supply. Once turned on, the glow plug heated the surrounding gaseous mixture to its auto-ignition temperature in less than 5 seconds. This system was effective down to a pressure of $4.0 \mathrm{kPa}$. A NI SignalExpress program was 
written to trigger the camera(s) and DAQ system when the lead shock passed the first pressure transducer (PT 1, shown in Figure 3.4). The sharp pressure-rise from this transducer was used to trigger the data acquisition and SignalExpress outputted a 5V TTL pulse to the camera. The cameras were set to 'centre trigger mode'. The cameras continuously record to a buffer, until they receive the trigger signal at which point the recording time is divided equally before and after the trigger signal.

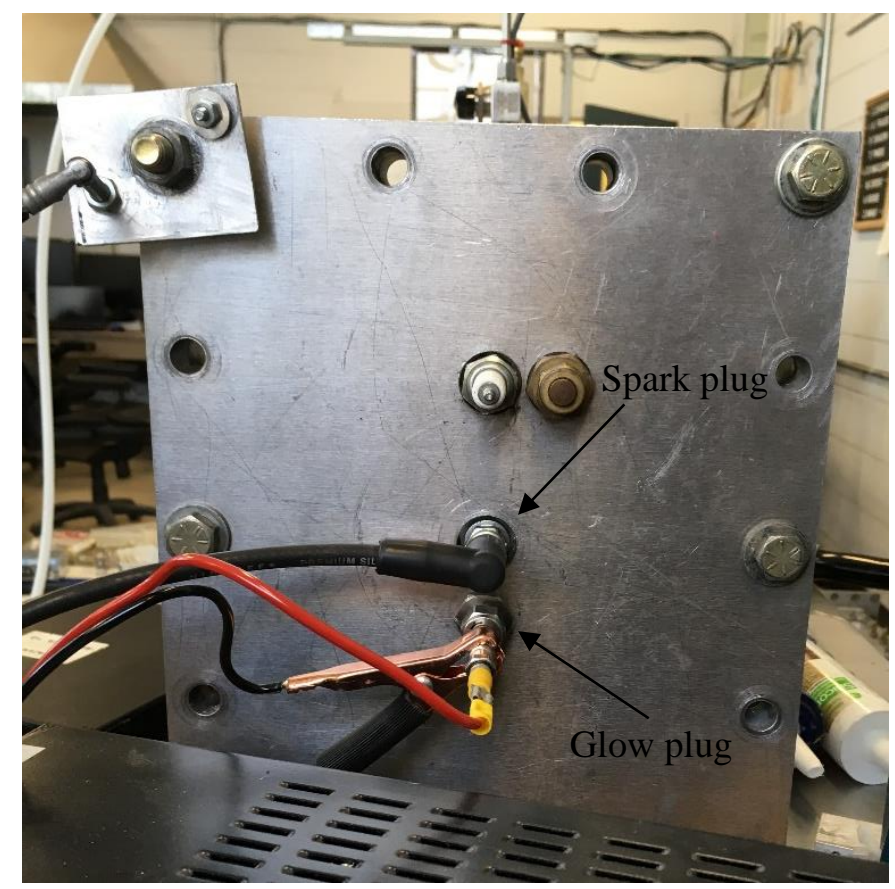

Figure 3.10: Ignition flange showing the position of the spark and glow plug ignition systems

\subsection{High Speed Photography}

Two high-speed CMOS cameras were used to obtain self-luminous images. A Photron SA-Z was used to record video through the optically clear tube. A Photron SA-5 was used to record video through the glass window in the end-flange (or additional side-view video with a different field-of-view). FastCam Viewer software was used to synchronize the two cameras. The Photron SA-Z was used as the 'master' camera, generating a TTL trigger output signal to the Photron SA-5 'slave' camera. 


\subsubsection{Camera Configurations}

Four camera tests with different fields-of-view were used to observe quasi-detonation propagation in the round tube. The field-of-view and camera configurations used for each of these test types are outlined in Table 3.2. The shutter speed was varied from $0.16 \mu \mathrm{s}$ to $5.00 \mu \mathrm{s}$, depending on the initial pressure (higher initial pressure produces more chemiluminescence). A faster shutter speed allows for a more precise location of the combustion front as it generates a sharper image with less blurring. However, it also generates a darker image as less light reaches the camera sensor. In some cases, this resulted in not being able to see the combustion front to track it. Therefore, for each initial pressure, the fastest shutter speed which still provided adequate light was used. For tests producing very bright combustion and where the shutter speed was set to the lowest setting $(0.16 \mu \mathrm{s})$, a neutral density filter was used to reduce the incoming light and prevent oversaturation of the images. 
Table 3.2: Camera Configurations

\begin{tabular}{|c|c|c|}
\hline Configuration & Properties & Field-of-view \\
\hline $\begin{array}{c}\text { Full- } \\
\text { view/Side- } \\
\text { view }\end{array}$ & $\begin{array}{c}\text { Camera: } \mathbf{S A - Z} \\
\text { Frame rate: } 70,000 \mathrm{fps} \\
\text { Resolution: } 1024 \times 280 \text { pixels } \\
\text { Field of view: } 5 \text { obstacles } \\
(24.1 \mathrm{~cm} \times 7.6 \mathrm{~cm})\end{array}$ & Field-of-v \\
\hline One obstacle & $\begin{array}{c}\text { Camera: } \mathbf{S A - Z} \\
\text { Frame rate: } 175,000 \mathrm{fps} \\
\text { Resolution: } 256 \times 280 \text { pixels } \\
\text { Field of view: } 5 \text { obstacles }(8.9 \\
\mathrm{cm} \times 7.6 \mathrm{~cm})\end{array}$ & 00 Field-of-vie \\
\hline $\begin{array}{c}\text { Channel } \\
\text { centreline slit }\end{array}$ & $\begin{array}{c}\text { Camera: } \text { SA-Z } \\
\text { Frame rate: } 300,000 \mathrm{fps} \\
\text { Resolution: } 1024 \times 56 \text { pixels } \\
\text { Field of view: } 5 \text { obstacles } \\
(24.1 \mathrm{~cm} \times 1.5 \mathrm{~cm})\end{array}$ & 900000 \\
\hline
\end{tabular}




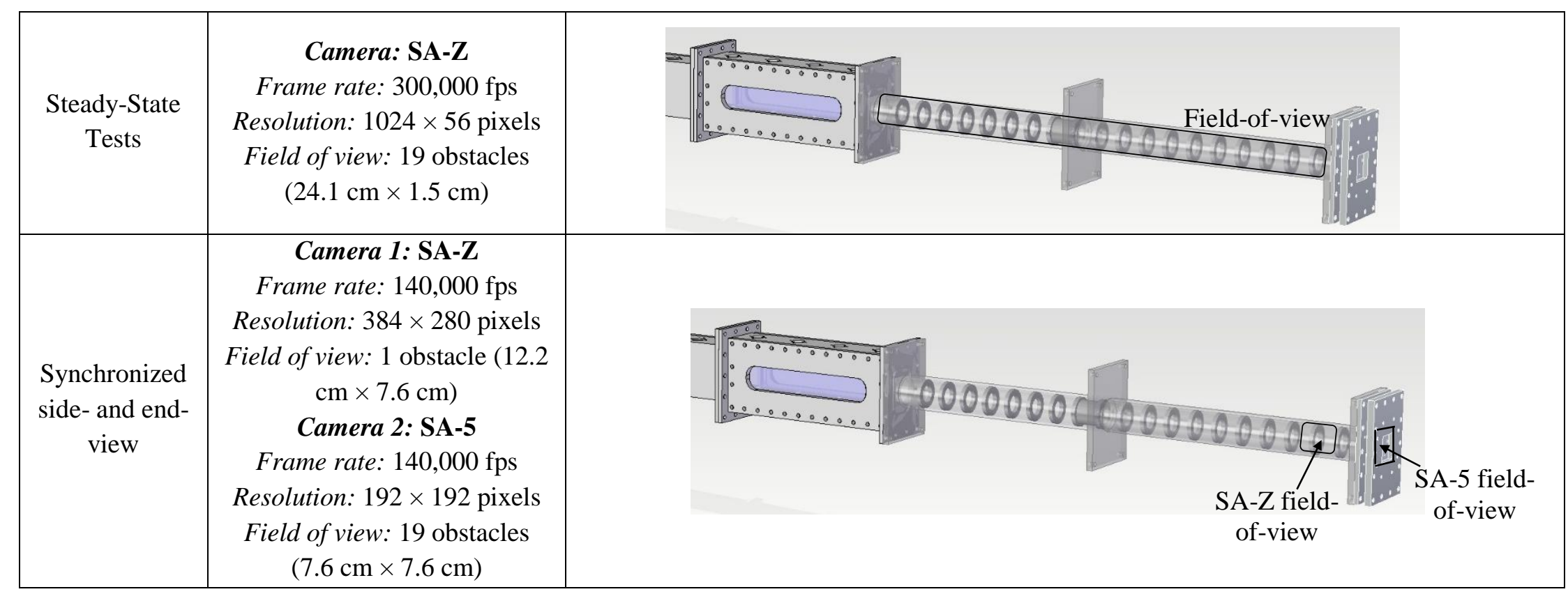




\subsubsection{Image Processing}

Photron FASTCAM Viewer ${ }^{\mathrm{TM}}$ was used to process the video data. The velocities from the fullview, channel centerline slit and obstacle pair configurations were processed using the following method. The crosshair feature was used to manually determine the position of the front pixel in the combustion wave along the channel centerline which was recorded in Excel with the time stamp. The pixel density in metres per second, $\alpha$, was calculated by measuring the number of pixels across the edge-view of an orifice plate and dividing by the known distance. The movement of the combustion front between frames, $\Delta p x$, measured in pixels, was converted to a distance by multiplying by the pixel density. The average combustion front velocity along the channel centerline, $\mathrm{v}_{\mathrm{c}}$, was determined according to

$$
v_{c}=\frac{\alpha(\Delta p x)}{\Delta t}
$$

The average velocity in the 'full-view' configuration was obtained over the last five obstacles in the channel taking one combustion front position between orifice plates 14 and 15 and one combustion front position and time between orifice plates 17 and 18 . The combustion front velocity was determined between each frame for the channel centerline slit and obstacle pair tests, so there were multiple local velocities between obstacles. The uncertainty associated with these measurements is presented in Appendix B.

The images from the steady-state tests were processed differently. The time of impact when the front of the combustion wave collided with the front surface of the orifice plate was recorded. Given the $7.62 \mathrm{~cm}$ distance between orifice plates, an average combustion front velocity between each set of orifice plates was calculated. The entire tube was filmed in this configuration and these velocities were used to develop velocity profiles along the length of the tube. The uncertainty associated with these measurements is presented in Appendix B. 


\subsection{Soot Foils}

Aluminum soot foils were used to record the detonation structure at two locations in the round tube. Curved foils, installed along the channel's inner wall, recorded the detonation structure at the wall and the flat foils recorded the centerline structure. The foils were cut from thin 6061 aluminum sheets and lightly sooted using a kerosene lamp.

The curved foils were formed from $6.35 \mathrm{~cm} \times 23.88 \mathrm{~cm}$ pieces of aluminum and rolled using a hand roller. They were installed along the inside circumference of the acrylic spacers, between orifice plates 9 and 10, as shown in Figure 3.11.

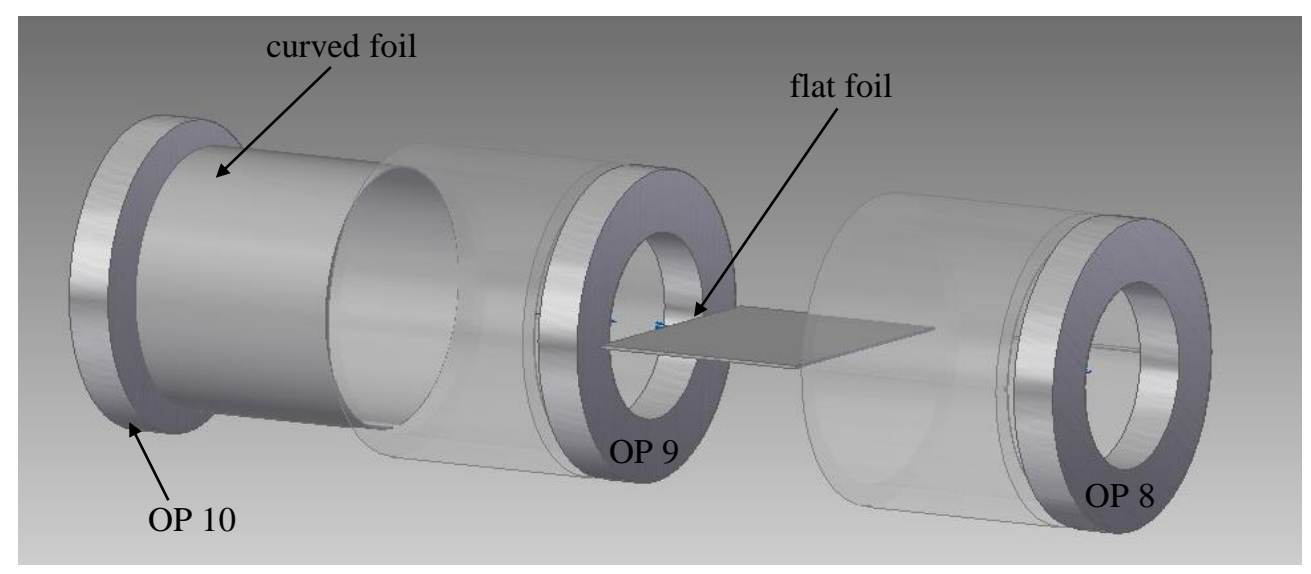

Figure 3.11: Exploded view of Solid-Edge model showing how the curved and flat foils are installed in the channel

The flat foils were installed along the channel centreline between orifice plates, as shown in Figure 3.11. These $6.35 \mathrm{~cm} \times 7.62 \mathrm{~cm}$ foils were installed in both a vertical and horizontal orientation. An acrylic spacer was scored lengthwise, so that the foil was supported on both sides and remained in place. Flat foils were only used for pressures greater than $30 \mathrm{kPa}$, as they are only useful for a detonation that is planar and perpendicular to the foil.

After each test, the soot foils were removed, sprayed with a clear paint (Rust-oleum Painter's Touch Clear Satin) to preserve the soot and photographed under an LED bank of lights using a digital camera. 
The soot foil tests were completed in the single segment round tube due to the difficulty in disassembling, reassembling and resealing the double-segment channel. The steady-state tests confirmed that the flow had reached steady-state for these tests.

\subsection{Operating Procedure}

Before running a test, the ignition and camera systems are first set-up and tested. The appropriate camera settings are selected, based on the configurations outlined in Section 3.4.1, and a background light calibration is performed. The ignition system power supplies are turned on and the SignalExpress ignition and DAQ program are tested to ensure that the system is triggering and the data is collected. Next, the mixing chamber pressure transducer is checked to ensure that there is a viable mixture in the mixing chamber. The mixing chamber must have a positive gauge pressure to ensure that air is not drawn into the mixing chamber. Once the pressure in the mixing chamber falls to atmospheric pressure, a new mixture must be made.

Before making a new mixture, the old mixture must be safely disposed of. This is achieved by diluting the mixture with air and venting it out of the building. House air is added to the tank until the tank pressure reads $300 \mathrm{kPa}$ (Valves 5 and 1 are opened), then the house air (Valve 1) is closed and the control panel exhaust (Valve 4) is opened. The exhaust (Valve 4) is closed and the process is repeated at least five times. The combustible mixture is now sufficiently diluted that it is safe to evacuate the mixing chamber into the lab. The oxygen and hydrogen lines are purged by briefly flowing their respective gases through them to remove all air from the line (Valve 2 and then Valve 3 open to Valve 4). The mixing chamber and control panel is evacuated using the Edwards model E2M0.7 vacuum pump for at least an hour. This is achieved by closing all the valves except Valve 7 and 5. Once the mixing chamber has been vacuumed, the vacuum valve (Valve 7) is closed. Valve 3 is opened until $200 \mathrm{kPa}$ of hydrogen is added to the mixing chamber and then closed. Valve 2 is opened to add $100 \mathrm{kPa}$ of oxygen to the mixing chamber and then closed. The tank is closed (Valve 5), the gas bottles closed and the impeller is turned on for thirty minutes to mix the gases. 
The combustion channel was evacuated for at least 30 minutes or until the channel pressure transducer (P1) showed $0.0 \mathrm{kPa}$. This was achieved by opening Valves 6, 7, 8 and 9 . For very low pressure tests, $(\mathrm{Pi}<10 \mathrm{kPa})$ the chamber was vacuumed for at least one hour. Once the vacuuming time has elapsed, Valve 7 is closed. At this point, eye and ear protection is put-on and is worn while filling the channel with the combustible mixture and for the duration of the test. The combustible mixture is loaded into the combustion channel by opening Valve 5, until the channel pressure transducer read the desired pressure. To run a test, all open valves are closed (Valves 6 and 8), isolating the channel. As the pressure transducer is not rated for above atmospheric pressures, the pressure transducer gauge (Valve 9) is also closed.

For the spark triggered tests, the cameras were set to record and the "NI SignalExpress trigger" program was run, igniting the mixture. For the glow plug tests, the cameras were set to record and the "pressure transducer triggered NI SignalExpress DAQ program" was run. The glow plug power supply was manually switched on until autoignition of the mixture occurred.

At the end of each test, the combustion products were purged from the channel using house air and by opening Valves 1, 6, 8 and 10. This is done for two reasons: to remove the combustion products (which contains mainly water vapour), and to dilute and purge the mixture if combustion was not initiated. Purging lasts approximately 5 minutes and then, Valves 1 and 10 are closed. The final step is opening Valves 7 and 9 to evacuate the combustion channel for the next test. Eye and ear protection may be removed at this point.

For tests where the optical end flange was used instead of the dump tank, there was no combustion channel exhaust (Valve 10). In these tests, the tube was vented to atmospheric using the control panel (Valve 4) and then evacuated (where Valve 4 was closed, and Valves 7 and 9 opened). 


\section{Chapter 4}

\section{Results and Discussion}

The results are organized into two sections, based on the cylindrical channel blockage ratio used in the experiments.

\subsection{0\% Blockage Ratio Results}

This section presents the results for the $50 \%$ BR orifice plates in the cylindrical tube. Tests were performed using stoichiometric hydrogen-oxygen mixtures at initial pressures of $6.0 \mathrm{kPa}$ to $60 \mathrm{kPa}$. In this configuration, the $50 \%$ blockage ratio in the cylindrical channel matches that of the square channel, but has an opening diameter of $5.33 \mathrm{~cm}$ compared to the $3.81 \mathrm{~cm}$ opening in the square channel obstacles.

\subsubsection{Steady-State Behaviour}

Following ignition, a flame accelerates in the square channel [8] reaching a steady velocity before entering the cylindrical tube. Due to the perturbation caused from the change in geometry, the combustion front re-establishes itself in the cylindrical channel. A series of tests were conducted to verify that there was sufficient distance for the front to re-establish in the round tube so that steady-state behaviour and sustainable propagation modes were studied over the field-of-view in the round tube. These tests were completed in the two-segment cylindrical channel equipped with 19 orifice plates. The combustion front was tracked along the length of the cylindrical channel to develop a velocity profile, according to the procedure outlined in Section 3.4.2. Additional information on this configuration is provided in Section 3.4.1.

The 'steady-state' experiments were completed over a range of initial pressures $\left(\mathrm{P}_{\mathrm{i}}\right)$ between 6 and $40 \mathrm{kPa}$, the results of which are compiled in Figure 4.1. The $\mathrm{x}$-axis represents the distance from the squareto-cylindrical transition and is recorded in terms of the orifice plate number, where the distance between orifice plates is $7.6 \mathrm{~cm}$. The average velocity between orifice plates $(\mathrm{OP})$ is plotted at the second orifice plate of the pair (i.e. the average velocity between OP 4 and OP 5 is plotted at OP 5). The average velocity 
in the last section of the square channel (calculated according to Section 3.3.1) is plotted at Obstacle 0. For clarity, only a selection of the test results are presented. The CJ detonation velocity and speed of sound in the products, averaged over the pressure range, are also plotted for reference. Over this pressure range, the change in CJ velocity and combustion products speed of sound is only $130 \mathrm{~m} / \mathrm{s}$, and $55 \mathrm{~m} / \mathrm{s}$, respectively.

Steady-state tests were not completed at initial pressures greater than $40 \mathrm{kPa}$, because combustion propagation stabilizes more quickly at higher pressures and it was clear that the velocity was already approximately constant for tests with initial pressures greater than $25 \mathrm{kPa}$. Tests with initial pressures lower than $6.5 \mathrm{kPa}$ were not filmed in this configuration as there was insufficient light to track the combustion front. For clarity, error bars are not shown on the plot, but each velocity has an associated error ranging from approximately $\pm 50 \mathrm{~m} / \mathrm{s}$ for the $6.5-10 \mathrm{kPa}$ tests up to approximately $\pm 250 \mathrm{~m} / \mathrm{s}$ for the $25-40 \mathrm{kPa}$ tests. The full uncertainty analysis is provided in Appendix B.

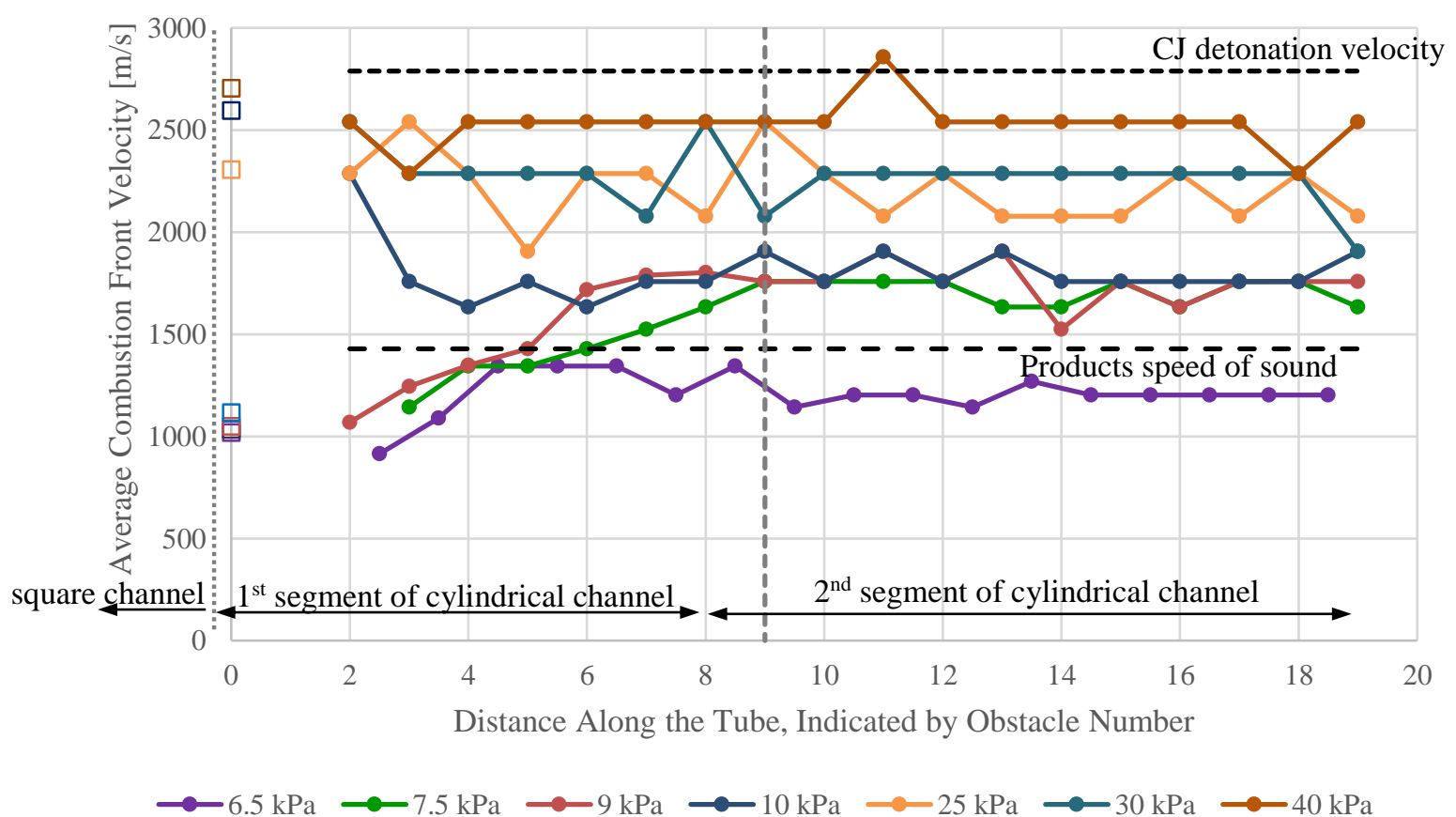

Figure 4.1: Plot of velocity profiles along the cylindrical channel for 50\% BR for a range of initial pressures 
The following observations were made based on the steady-state tests:

- For high pressure tests $\left(\mathrm{P}_{\mathrm{i}}>15 \mathrm{kPa}\right)$, the combustion wave propagated along the tube at an approximately constant velocity. Any acceleration or deceleration from the square channel occurred between the start of the cylindrical channel and the first obstacle, which could not be captured due to the camera field-of-view.

- For tests performed at an initial pressure of between 10 and $15 \mathrm{kPa}$, the combustion wave decelerated after the square-to-round transition, but stabilized to a quasi-steady velocity within the first three obstacles.

- For lower pressure tests $(7-9 \mathrm{kPa})$, where a fast-flame (which propagates at an average velocity below the combustion products speed of sound) exits the square channel, acceleration up to a quasi-steady detonation (which propagates at an average velocity above the combustion products speed of sound) occurred within the first ten obstacles. As the initial pressure decreased within this pressure range, acceleration from a fast-flame to a quasi-steady detonation occurred over a longer distance (for example at $9 \mathrm{kPa}$, the acceleration occurred within the first 6 obstacles, but took 9 obstacles for the $7.5 \mathrm{kPa}$ test). However, even at the lowest pressure, the combustion wave reached a quasi-steady propagation mode by Orifice Plate (OP) 14, the start of the optical field-of-view.

- For the lowest pressure tests $(6-6.5 \mathrm{kPa})$, a fast-flame exited the square channel and continued to propagate down the cylindrical channel as a fast-flame at a quasi-steady velocity.

Preliminary tests were performed with only a single cylindrical channel segment, equipped with 10 orifice plates. A second segment was added to the cylindrical channel, effectively doubling the round tube length, to ensure that steady-state behaviour was studied. Based on the results of the 'steady-state tests', steady-state propagation was observed for all pressures over the optical field-of-view (OP $14-19)$ in the 
two-segment channel. In the one-segment channel, steady-state propagation was observed in the optical field-of-view (OP 5 - 9) for initial pressures of $9 \mathrm{kPa}$ and higher.

\subsubsection{Comparison of Cylindrical and Square Channel Combustion Front Velocities}

A plot of the average combustion front velocity in the square and cylindrical channels as a function of initial pressure is presented in Figure 4.2. For each test, there is a velocity in the square channel (indicated by a square marker) and a corresponding velocity in the cylindrical channel (indicated by a circular marker). The average velocity in the square channel and round tube was measured according to Section 3.3.1 and 3.4.2, respectively. For reference, the theoretical CJ detonation velocity and the speed of sound in the combustion products (calculated for an adiabatic isobaric process) are also plotted. The calculated CJ detonation velocities and speed of sound in the combustion products are summarized in Appendix D. For transparency, the one-segment and two-segment cylindrical channel tests are plotted separately. 


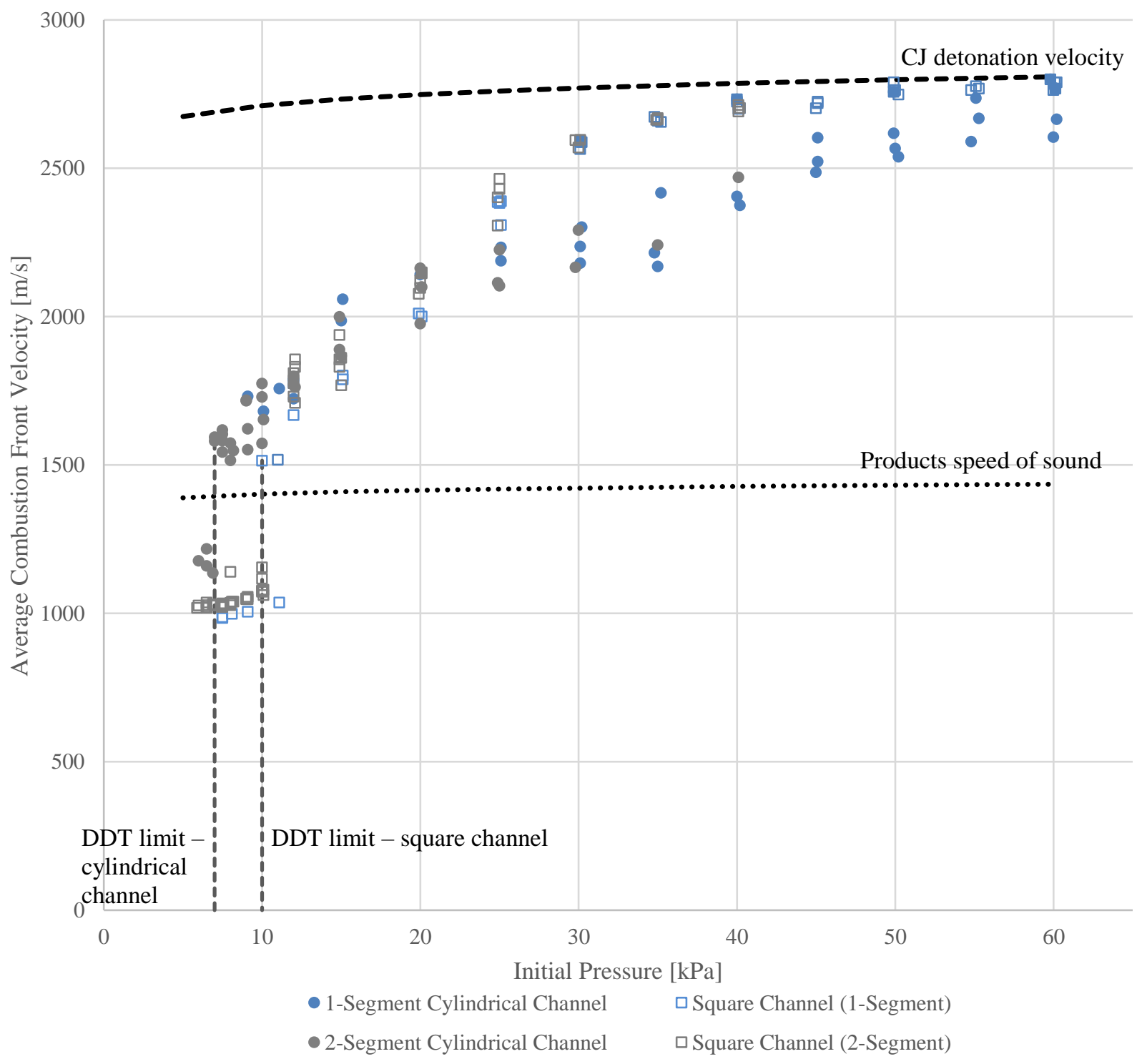

Figure 4.2: Plot of average combustion front velocity in the square and cylindrical channels as a function of initial pressure for a $50 \%$ blockage ratio

The velocity data in Figure 4.2 shows a sudden jump from values below the combustion products speed of sound to values above it. This increase is traditionally referred to as the DDT or detonation propagation limit, and signals the least reactive mixture in which DDT will occur. The detonation limits are shown on the plot by a vertical dashed line for each set of data. The detonation limit is $7 \mathrm{kPa}$ in the cylindrical channel, which is lower than the detonation limit of $10 \mathrm{kPa}$ in the square channel. This difference 
in the DDT limit is expected based on Peraldi's $\mathrm{d} / \lambda>1$ criteria [5] because the orifice diameter in the round tube $(5.33 \mathrm{~cm})$ is larger than the opening between the fence-type obstacles in the square channel $(3.81 \mathrm{~cm})$. Based on the cell size data presented in Appendix C, the limit in the square channel corresponds to $d / \lambda \approx$ 1.6 and the limit in the round tube corresponds to $d / \lambda \approx 1.4$. These values are consistent with $d / \lambda$ values reported in the literature at the DDT limit.

The independence of the round tube velocity data at $10 \mathrm{kPa}$ shows that propagation in the round tube is not sensitive to whether a detonation wave or fast-flame emerges from the square channel. For initial pressures between 12 and $25 \mathrm{kPa}$, the average velocity in both the square and round channels are very similar. However, for initial pressures greater than $25 \mathrm{kPa}$, the average velocity in the square channel is significantly higher than in the cylindrical channel. In fact, at an initial pressures greater than $50 \mathrm{kPa}$, the average propagation velocity in the square channel is approximately equal to the theoretical CJ detonation velocity, with very little variation from test-to-test. The difference in velocities may be attributed to the effect of curvature on detonation diffraction through the obstacles. In the square channel, diffraction only occurs at the top and bottom of the channel when the wave propagates around the fence-type obstacle, essentially a two-dimensional diffraction process. In the round tube, a three-dimensional diffraction process occurs around the circumference of the orifice opening. The three-dimensional diffraction (and associated higher curvature) in the orifice plate results in a faster decoupling of the detonation wave, and ultimately in a lower average velocity between orifice plates. For fast-flames, higher average velocities were observed in the round tube than the square channel. Since the energy released is not coupled to the lead shock in fastflames (turbulent transport is more important), the severity of the diffraction is not as important. As shown in [8], the interaction of the reflected shock with the flame governs the average flame velocity for fastflames.

\subsubsection{Propagation Modes in the Cylindrical Channel}

At initial pressures of $6.5 \mathrm{kPa}$ or less, a sustained choked flame (known more generally as a fastflame) was observed. Based on the video results, quasi-detonation propagation in the cylindrical channel 
can be characterized by three modes: single hot-spot wall ignition detonation ( $7-15 \mathrm{kPa})$, multi-hot-spot wall ignition detonation $(20-45 \mathrm{kPa})$ and continuous detonation $(50-60 \mathrm{kPa})$. Hot-spot ignition refers to the creation of a localized detonation kernel, typically occurring in a compressed turbulent region of hot, unburned gas produced by shock reflection off a boundary. A fourth transitory mode was observed where detonation initiation occurred on the obstacle face, but in all cases the combustion wave stabilized downstream in the tube into the single hot-spot ignition mode. In the next sections, each of these modes will be described based on video and soot foil records.

\subsubsection{Fast-Flame Regime}

Fast-flame propagation was observed for initial pressures less than $7 \mathrm{kPa}$. A fast-flame, which consists of an uncoupled shock-flame complex, is shown propagating in video frames (Figure 4.3) taken from a $6.5 \mathrm{kPa}$ test. The edges of the orifice plates appear as equally spaced black vertical strips, as indicated in Image 15. The flame emerges from OP 14 in Image 1 and propagates towards the next orifice plate in Images $2-4$. The light emitted from the flame is very faint and because this video was filmed using regular photography, the shock wave that is propagating ahead of the flame is not visible. The flame becomes noticeable when the reflected shock, originating from reflection of the incident shock off the upstream face of OP 15, traverses the flame, as seen in Images 5 and 6. This is more evident further downstream in Images $12-13$. The interaction of the shock with the flame stops the progress of the flame and the implosion of the reflected shock (indicated by the torus shape) behind the flame front forms a characteristic bright spot, as seen in Image 7 (and in Image 10). These repeating bright spots formed by a propagating fast-flame were also observed by Lieberman and Lee in their streak photography for experiments completed with a 1D orifice spacing (the same orifice spacing used in present research) [4].

A distinguishing feature of a fast-flame is that shock reflection, off the tube wall or obstacle face, does not result in detonation initiation and the combustion wave propagates at a velocity well below the speed of sound in the products. Fast-flame propagation in the round tube is similar to fast-flame propagation 
observed by Kellenberger and Ciccarelli [8] in a rectangular combustion channel where Schlieren photography highlighted all of the above features. 

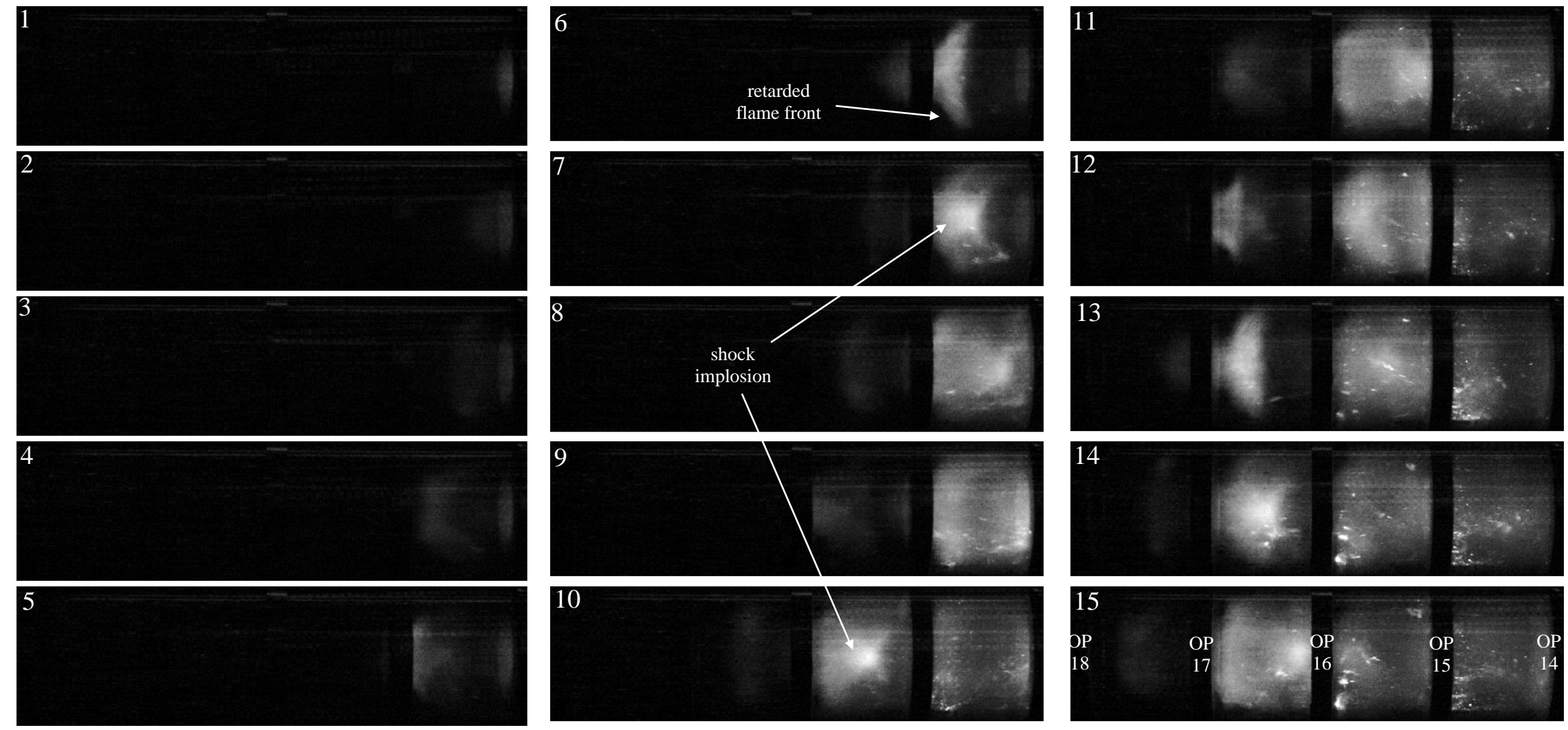

Figure 4.3: Full-view video for a $6.5 \mathrm{kPa}$ test with $50 \% \mathrm{BR}$, showing the fast-flame regime filmed at 70,000 fps (Test 225) 


\subsubsection{Single Hot-Spot Wall Ignition Detonation}

For test pressures between 7 and $15 \mathrm{kPa}$, a single detonation kernel formed at the tube wall between orifice plates. This pressure range corresponds to a $d / \lambda$ of 1.5 to 3.6 in the cylindrical channel. Figure 4.4 shows the synchronized side- and end-view video images from a test run at $10 \mathrm{kPa}$. The side-view spans one set of orifice plates, including the downstream-edge of OP 8 (as marked by the vertical white line) and the upstream-face of OP 9 illuminated in Images 5a and 6a. The end-view allows for the detonation front to be tracked as it interacts with the orifice plate face, producing light that can be observed through the Acrylic orifice plates down the length of the tube. Note the detonation wave cannot be observed in the core of the orifice plates due to excessive light emitted by the combustion products over the length of the tube. A bright spot (with accompanying arrow showing the propagation direction) indicates a detonation initiation at the top wall of the tube in Image 2a, which grows radially in Image 3a. The detonation wave collides with the top of OP 9 first, as seen in Image 4a, and spreads down and around the orifice plate's upstream surface, as indicated by the progression of the bright area seen in Images 5a and 6a. This progression around the orifice plate face is seen in the end-view and is marked by arrows in Images $4 \mathrm{~b}, 5 \mathrm{~b}$ and $6 \mathrm{~b}$. Due to the curvature of the tube wall the detonation wave is focused on the opposite side of the tube from where it was initiated, producing the bright light associated with the eroded plastic from the bottom of OP 9 in Image 6 a. 


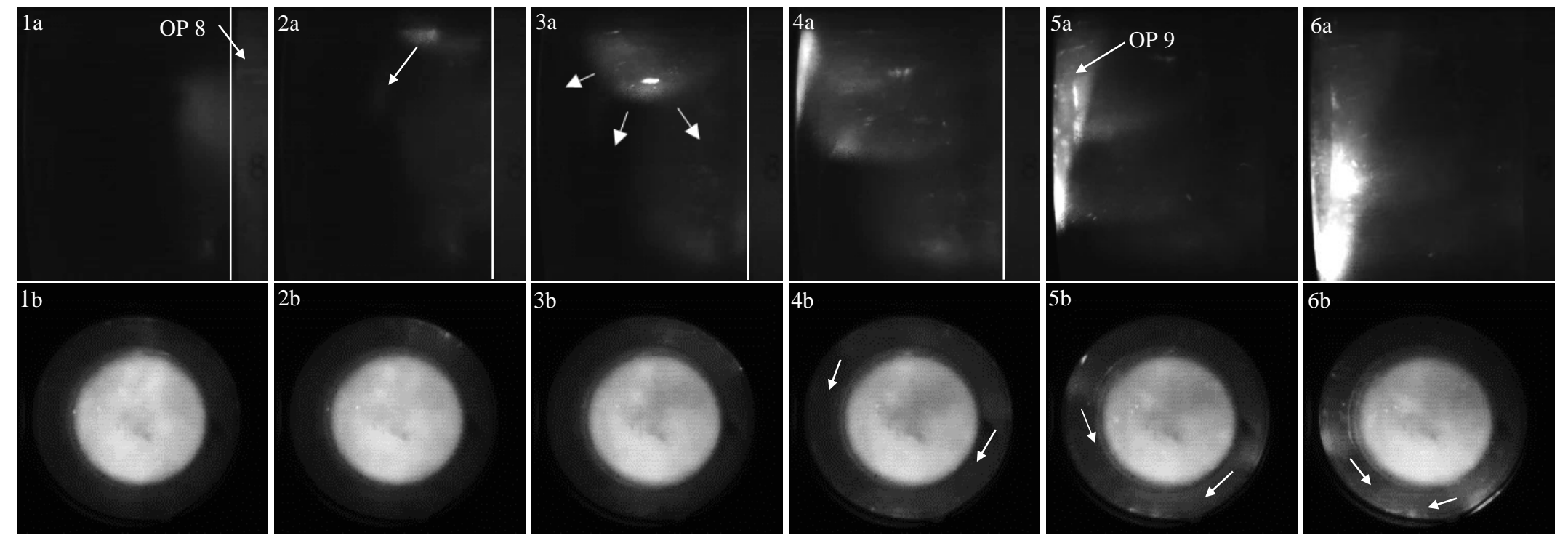

Figure 4.4: Video from a $10 \mathrm{kPa}$ test with $50 \%$ BR (Test 86), filmed at 140,000 fps. Top: Side-view video with field-of-view between OP 8 and 9, wave propagation right to left; Bottom: End-view video 
This propagation mode is also characterized by 'slapping wave' behaviour, where ignition points occur at alternating, diametrically opposite locations down the length of the tube. Therefore, as ignition occurred at the top of the tube between OP 8 and 9 (shown in Figure 4.4), one would expect that the wave transmitted through OP 9 would be biased in strength to the bottom of the tube between the next set of orifice plates (OP 9 and 10) and this would be the next ignition site. A curved soot foil positioned between OP 9 and 10, provided in Figure 4.5, confirmed that a detonation was initiated at a single point at the bottom of the tube on the tube wall. The detonation initiation site is circled.

When analyzing 'curved' foils such as the one in Figure 4.5 , it is important to remember that although it was photographed after it was flattened, it was originally curved and installed between orifice plates around the circumference of the tube walls. The middle of the foil was installed at the top of the tube and where the left and right edges wrap around and meet was installed at the bottom of the tube. Note, the soot foil does not represent a snapshot at a given time, rather it provides a record of the path taken by a detonation wave. The foil shows a single ignition site after OP 9, roughly at the bottom of the tube (indicated by white circle). The two major triple-point trajectories (indicated by arrows) are followed by a band of very fine cell structure. The fine cell structure is caused by an overdriven detonation wave that propagates through the compressed unburned gas between the decoupled shock and flame. The smooth major triple point trajectory shows the position of the shock wave in time when traversed by the transverse detonation wave; and the irregular rear boundary of the fine cell region represents the position of the flame when traversed by the detonation wave. 


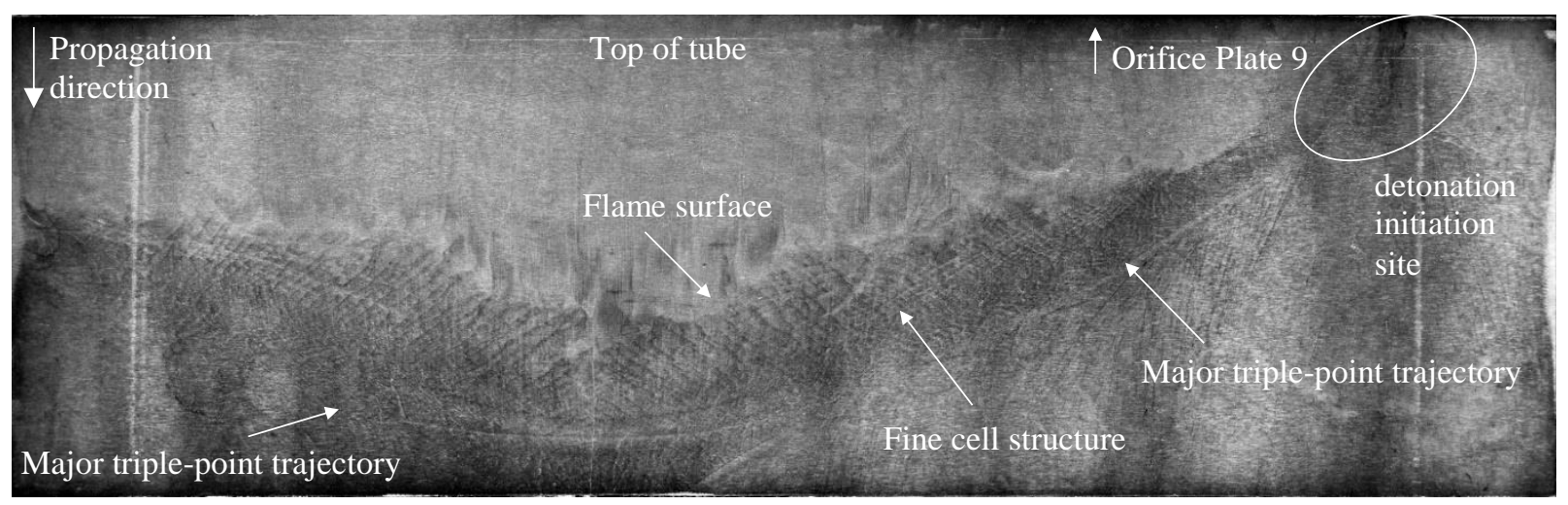

Figure 4.5: Curved soot foil obtained for a $10 \mathrm{kPa}$ test with $50 \%$ BR showing a single point of detonation initiation (Test 86)

An overview of the 'slapping wave' propagating down the entire second segment of the round tube in a $15 \mathrm{kPa}$ test is presented in Figure 4.6. Due to the distance between the camera and experimental apparatus, the detonation initiation sites on the tube wall and the propagation of the detonation around the obstacle face are difficult to see. However, the bright spots (highlighted by red circles in Images 4, 10, 15, $21,27,33,38,43$ and 49 ) which form when the two fronts of the combustion wave collide after spreading around the orifice plate, can be used to determine where ignition occurred. The bright spots form on the tube wall opposite the hot-spot ignition site (i.e. in Image 33, where the bright spot forms on the bottom of the tube, a hot spot would have formed at the top of the tube).

It is important to note that hot-spot ignition in the slapping wave does not only occur on the top and bottom of the tube. The slapping mode was observed in other configurations such as 'slapping' from the front to back of the tube and at other angles. However for presentation purposes it is easiest to visualize when detonation initiation occurs at the top and bottom of the tube. 

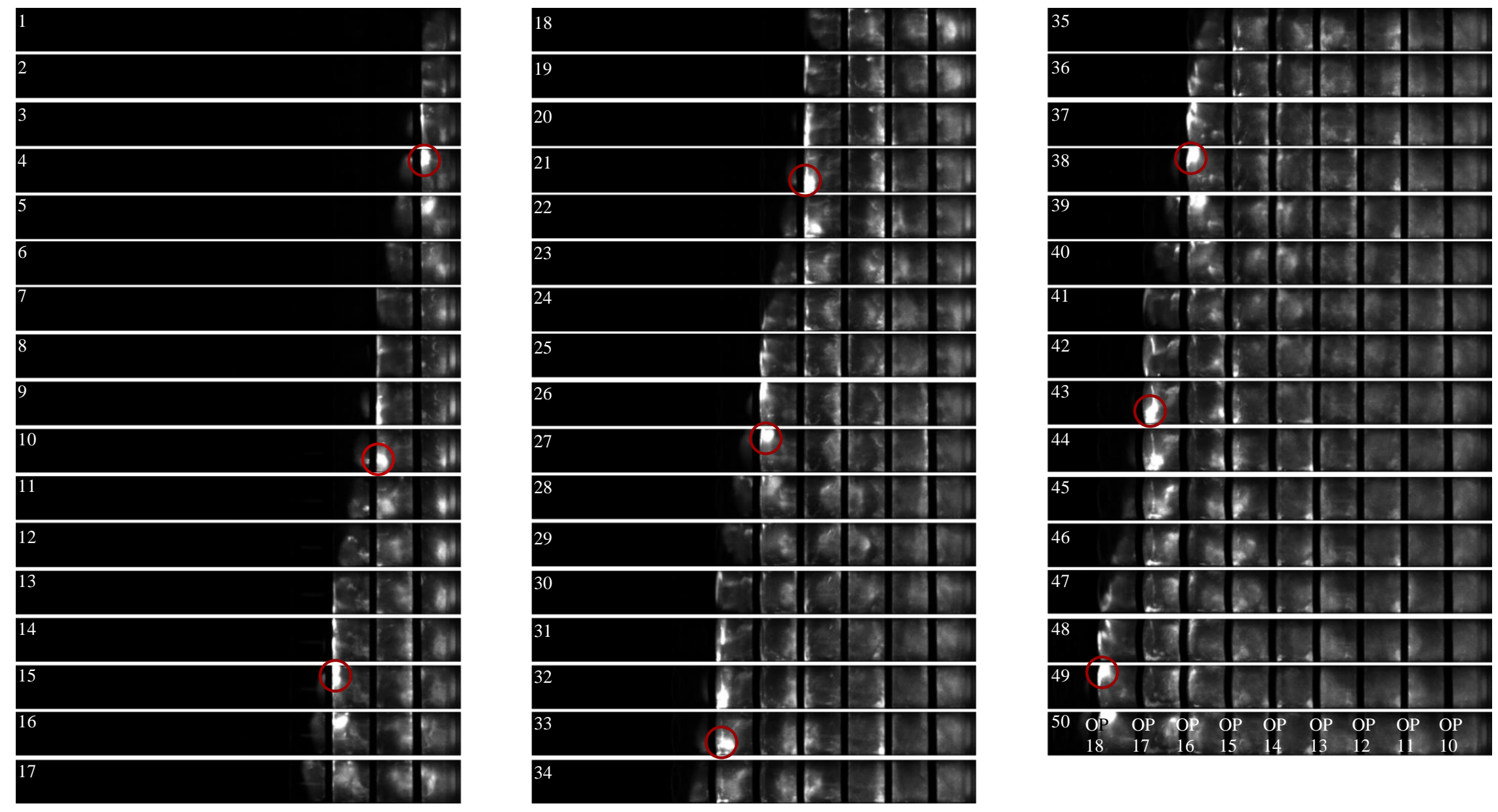

Figure 4.6: Side-view filmed at $\mathbf{3 0 0 , 0 0 0} \mathrm{fps}$, but only every fourth image is presented shown of the single wall hot spot ignition detonation for a $15 \mathrm{kPa}$ test with $50 \%$ BR (Test 192) 
Figure 4.7 shows a schematic of the observed mechanism of a single wall hot-spot ignition detonation. As the detonation passes through the orifice plate, it fails due to diffraction and the leading shock (shown in black) decouples from the flame (shown in red). The detonation is not planar as it enters and emerges from the orifice plate, and is biased in one direction (in the schematic, the detonation is first biased towards the top of the tube as indicated by the arrow). This directional bias influences the shock strength and ultimately where detonation initiation occurs. For example, a detonation biased towards the top of the tube will undergo the least diffraction towards the top of the tube, and therefore the shock will be strongest there. Conversely, the detonation will undergo the greatest diffraction in the opposite direction (in this case the bottom of the tube), resulting in the weakest shock and the largest separation distance between the lead shock and flame there.

As the detonation diffracts, the leading shock collides with the tube wall and reflects, generating a high reflected temperature and pressure. The shock reflects off the wall and propagates back into the preheated region of unburned gas between the leading shock and flame. A single local explosion (indicated by the red star at the top of the tube in Figure 4.7c) is initiated within this high-temperature, high-pressure region of unburned gas at the wall where the shock is strongest (this is the top of the tube in the schematic).

The detonation will spread, colliding first with the part of the obstacle face which it is closest to (in the case of the schematic, a detonation is initiated at the top of the tube first and will collide with the top of the orifice plate face first). The detonation wave will spread around the orifice plate face and the two heads of the detonation will collide at the bottom of the orifice plate. The detonation, which spreads radially, will be biased towards the bottom of the tube as it passes through the next orifice plate. As in the mechanism described above, the shock strength will now be greatest at the bottom of the tube and a detonation will be initiated at a single point on the tube wall at the bottom of the tube. For clarity in this two-dimensional sketch, detonations are shown as initiating at the top and bottom of the tube but can occur in any opposite directions in the tube. 


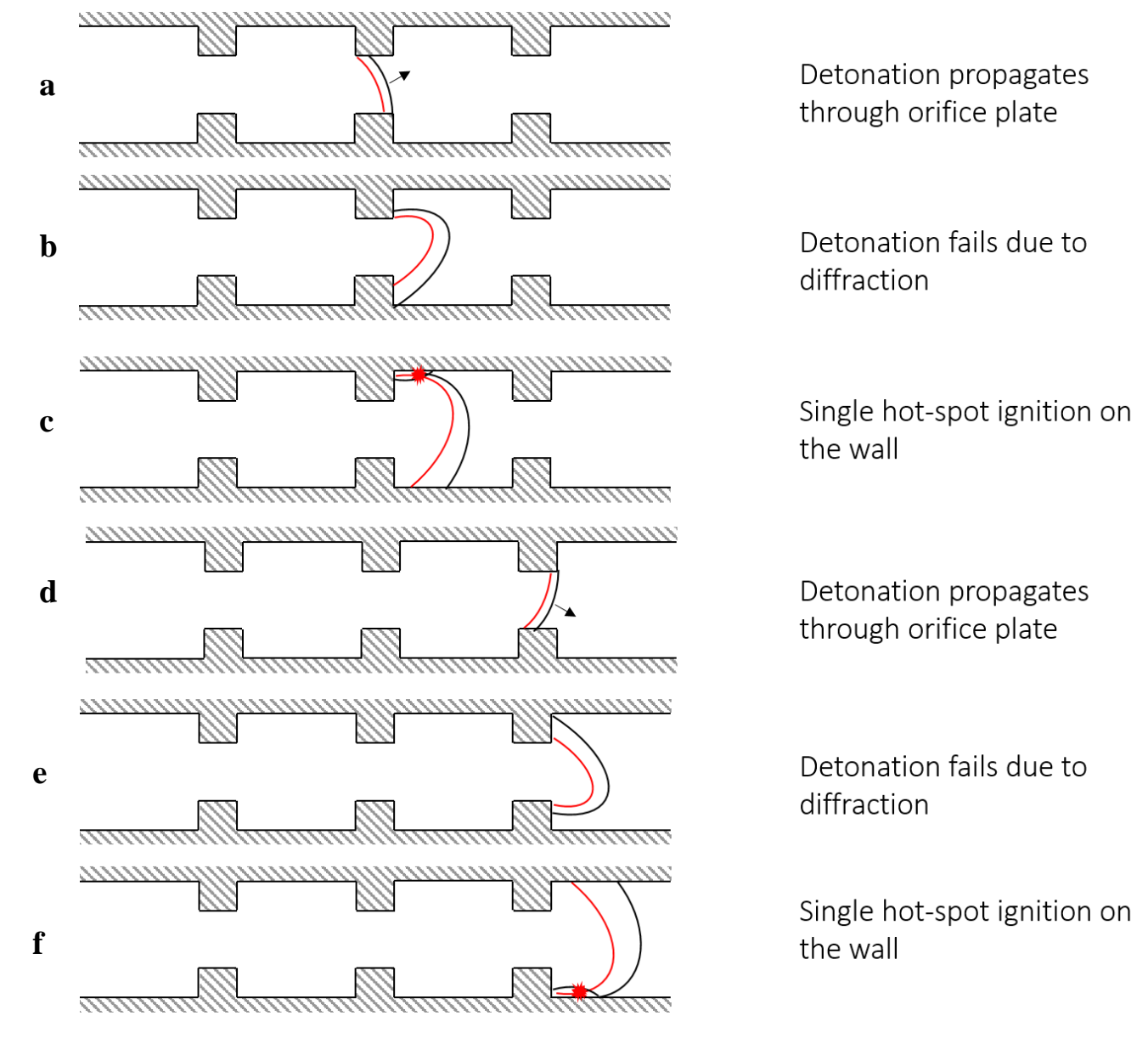

Figure 4.7: Schematic showing single hot spot wall ignition detonation mechanism propagating through the obstructed channel

\subsubsection{Multi-Hot-Spot Wall Ignition Detonation}

Tests with initial pressures between 20 and $45 \mathrm{kPa}$, yielded multiple detonation initiations along the wall of the cylinder. This pressure range corresponds to a $d / \lambda$ value of approximately 5 to 21 in the cylindrical channel. Side-view video footage showing multiple detonation initiation points (indicated by white arrows) on the tube wall for a $40 \mathrm{kPa}$ test is presented in Figure 4.8 . 

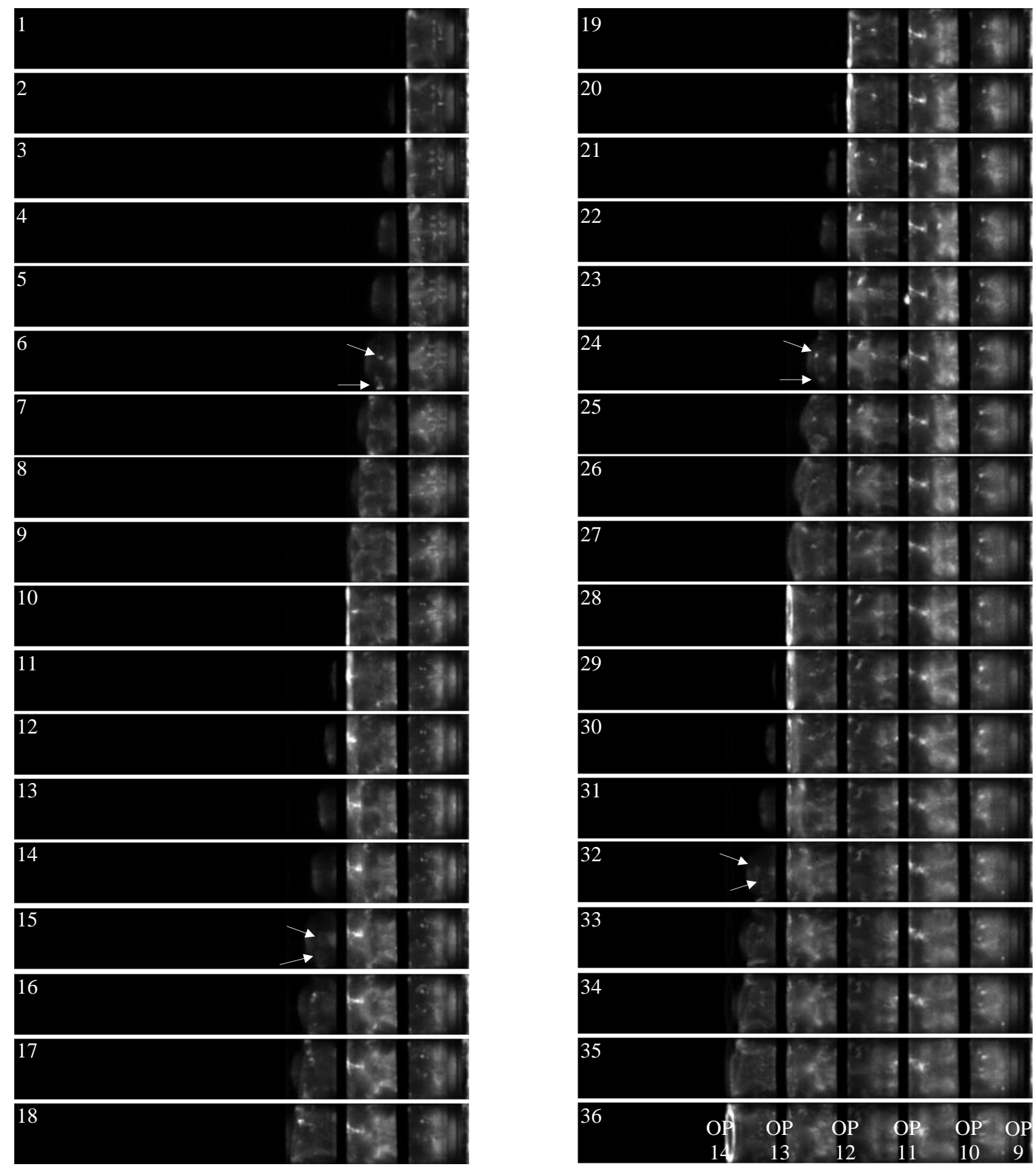

Figure 4.8: Side-view video footage of a $40 \mathrm{kPa}$ test with $50 \%$ BR showing multiple detonation initiation points on the tube wall, filmed at 300,000 fps (Test 204)

A close-up of detonation initiation at multiple hot-spots on the tube wall is presented in Figure 4.9, which show four side-view video images obtained for a $25 \mathrm{kPa}$ test. In the first two images the detonation 
wave fails after passing through OP 8 . After the decoupled shock (not observed in the image) reflects off the wall, two hot-spots form and develop into detonation waves (see arrows in Image 3). The exact location of the detonation initiation on the wall is difficult to discern from the images, but it is clear that detonation initiation occurs before shock reflection off the upstream-face of OP 9, which is just to the left of the fieldof-view.
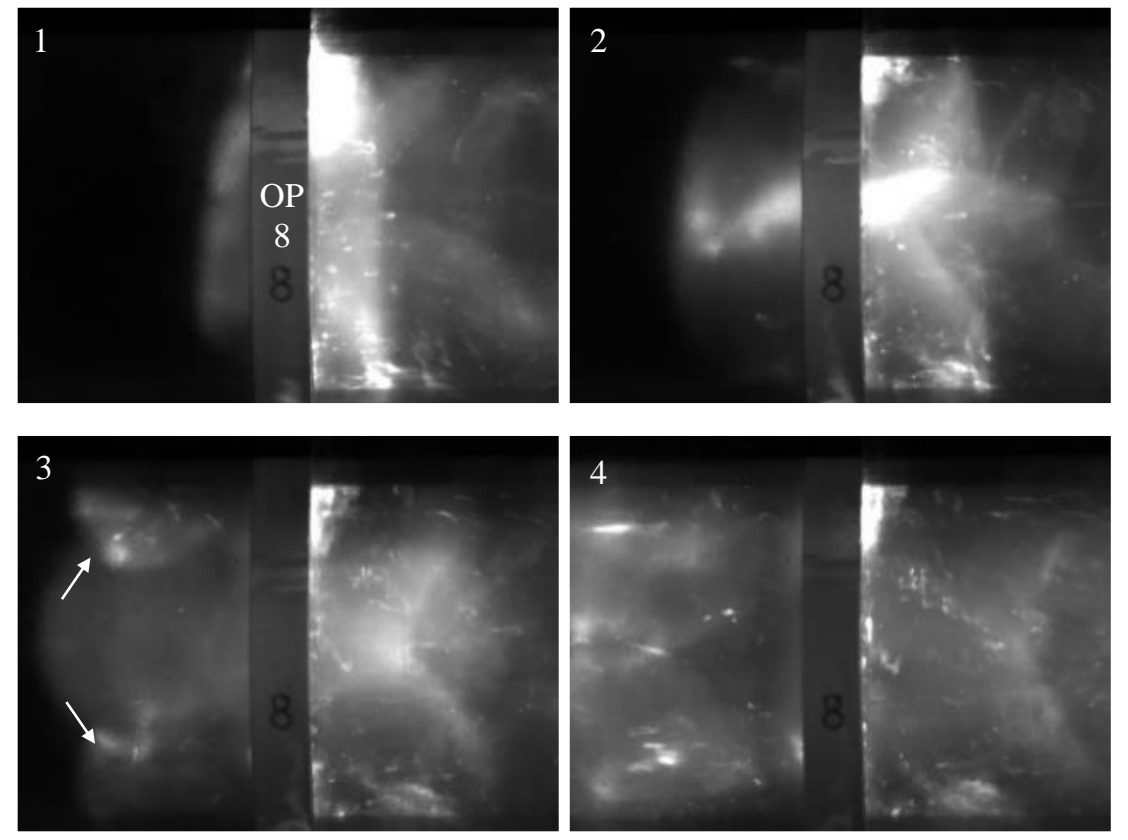

Figure 4.9: Images from a $25 \mathrm{kPa}$ test with a $50 \%$ BR (Test 75) showing detonation initiation from two hot spots on the tube wall, filmed at $140,000 \mathrm{fps}$

A curved soot foil for a $40 \mathrm{kPa}$ test (Figure 4.10) shows a cellular pattern typical of wall-reflection initiation of multiple detonation waves. Arrows on the foil mark the points of ignition. This foil is similar to the foils obtained from tests performed by Ciccarelli and Cross [6]. The absence of cells immediately after OP 9 indicates that the detonation fails after the orifice plate due to diffraction. In this test, there appears to be at least four hot spots that form into detonation waves. The detonation cells can be seen spreading from the detonation initiation sites. 


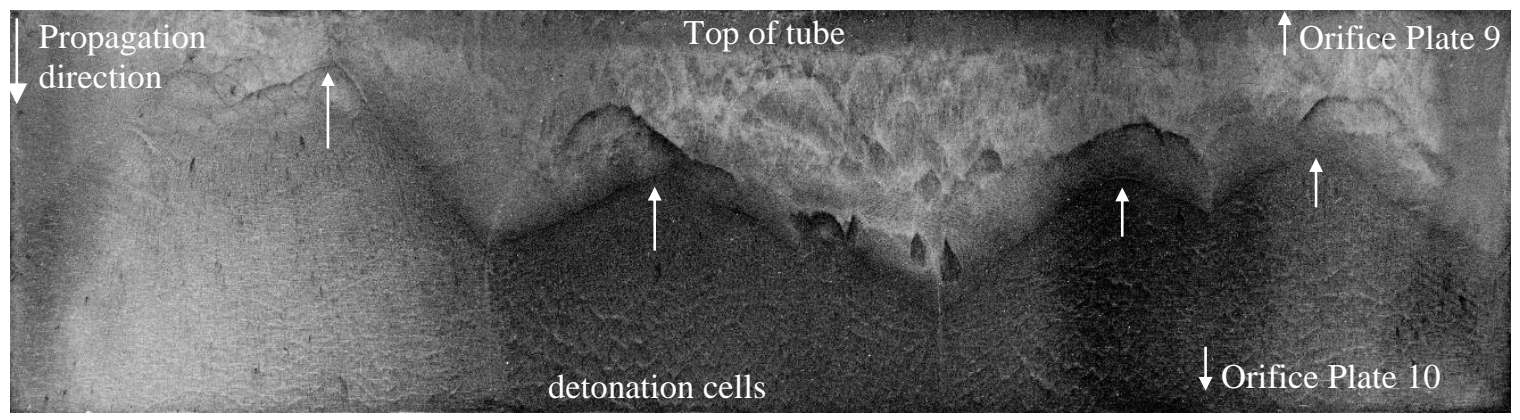

Figure 4.10: A curved soot foil for $40 \mathrm{kPa}$ test with $50 \% \mathrm{BR}$, showing multiple detonation initiation points (Test 158)

The multi-hot-spot wall ignition detonation mode is represented schematically in Figure 4.11. It is similar to the single hot-spot wall ignition detonation mode, and the detonation fails after diffracting through the orifice plate. The decoupled leading shock reflects on the tube wall, generating high temperatures and pressures. In the pre-compressed, unburned gas between the leading shock and flame, these conditions facilitate multiple detonation initiations on the tube wall. For clarity, only two detonations are shown on the schematic (on the top and bottom of the tube wall); however, detonations are initiated randomly at multiple points on the tube wall.
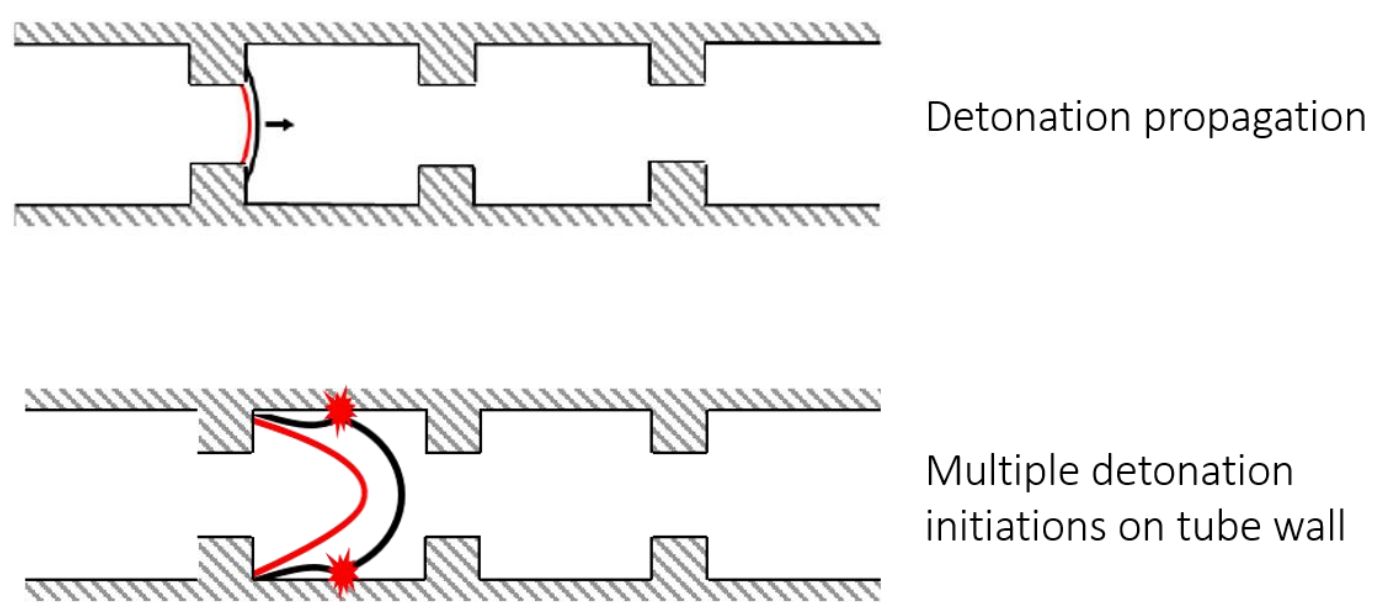

Figure 4.11: Schematic showing multi-hot spot wall ignition detonation propagation mechanism 


\subsubsection{Continuous Detonation $(50-60 \mathrm{kPa})$}

The continuous detonation regime is characterized by a detonation that does not fail along the channel centerline. This propagation mode was observed for initial pressures greater than $50 \mathrm{kPa}$, corresponding to a $d / \lambda>25$. The side-view footage obtained for a $60 \mathrm{kPa}$ test is shown in Figure 4.12, filmed at 70,000 fps. Based on this video, the velocity of the detonation wave along the channel centerline is $2614 \pm 86 \mathrm{~m} / \mathrm{s}$, with a standard deviation of only $24 \mathrm{~m} / \mathrm{s}$. As the detonation wave diffracts around the orifice plate the expansion causes it to fail along the tube circumference, so that a shock wave followed by a flame reaches the tube wall. The interaction of the shock and flame with the tube wall is discussed later.
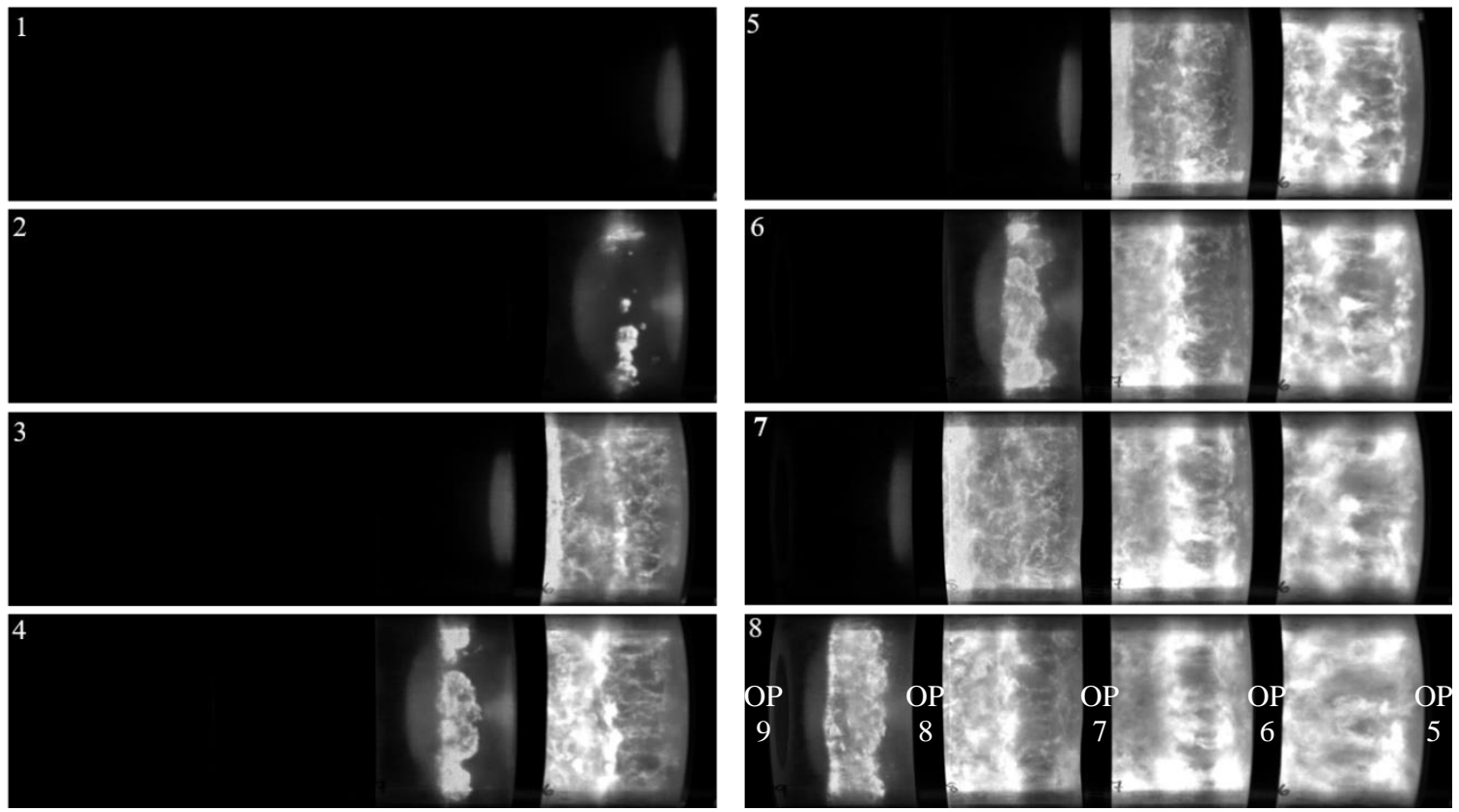

Figure 4.12: Side-view video footage of a $60 \mathrm{kPa}$ test with $50 \% \mathrm{BR}$, filmed at 70,000 fps (Test 153)

A flat soot foil for a $60 \mathrm{kPa}$ test, presented in Figure 4.13, shows continuous cells across the channel centerline. The soot foil was installed vertically between two orifice plates along the channel centerline (as shown in Figure 3.11) The cells along the centerline (the centreline is marked by a dashed line) are a 
consistent size and pattern, indicating that the detonation does not fail along the centerline due to diffraction. However, the significant velocity deficit relative to the CJ velocity (compared to the square channel data), seen in Figure 4.2 at $60 \mathrm{kPa}$, indicates that the global curvature induced by the diffraction impacts the velocity. The detonation cells outside the core, near the tube wall (seen at the top and bottom of the foil), are significantly larger than those along the centreline. Moving away from the core, the cell structure becomes less defined and eventually disappears, due to the decoupling of the detonation wave caused by the more severe diffraction turning the corner around the orifice plate inner-edge.

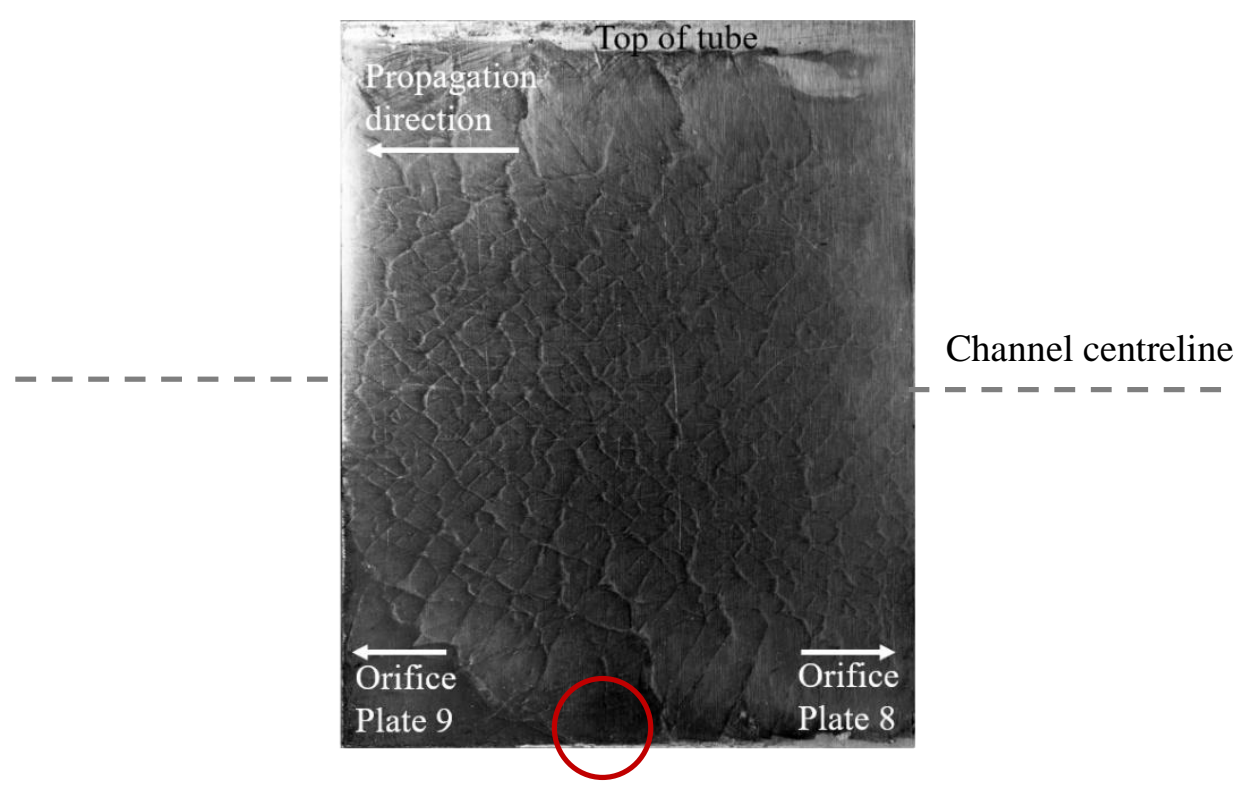

Figure 4.13: Vertical centreline soot foil installed for a $60 \mathrm{kPa}$ test with $50 \%$ BR (Test 157). Circle highlights where triple point interacts with tube wall.

A video captured for the same $60 \mathrm{kPa}$ test in which the soot foil was generated (soot foil shown in Figure 4.13) is shown in Figure 4.14. Filmed at 175,000 fps, the detonation is recorded propagating over the vertical soot foil, such that only the front half of the channel is shown. In all the images, the combustion front is very bright along the channel centreline, indicating the region of highest temperature. This bright, high temperature region is generated by the portion of the front which is a detonation (confirmed by the presence of detonation cells in this region in Figure 4.13). The wave front is significantly less bright at the 
top and bottom of the channel (indicated by the arrows in Image 2) because the detonation has become decoupled in this region (and therefore less heat is released).
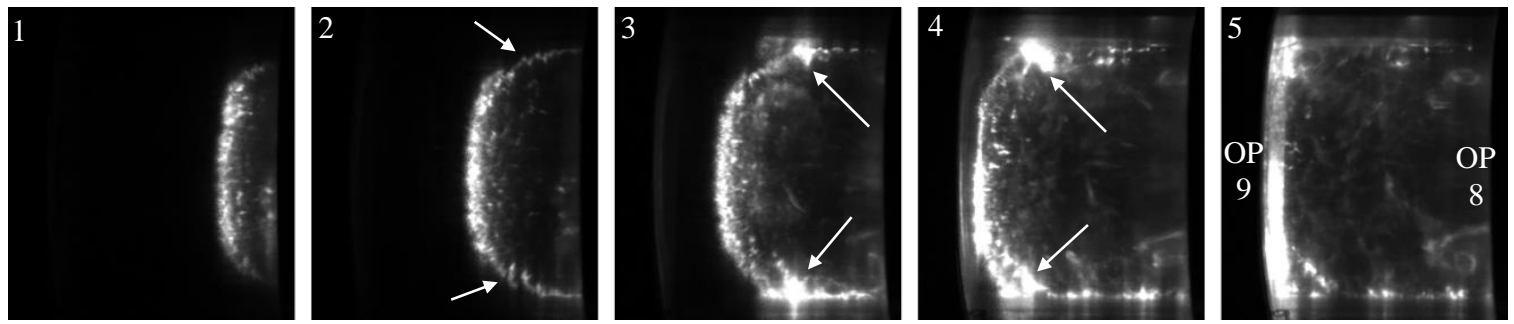

Figure 4.14: Video of $60 \mathrm{kPa}$ test with $50 \%$ BR recorded at 175,000 fps (Test 157). The soot foil presented in Figure 4.13 is installed vertically between OP 8 and 9, so the only the front half of the channel is visible.

The assertion of a constant centerline propagation velocity is further supported by the tracking of the detonation front along a narrow $(15 \mathrm{~mm}$ high) slit at the channel centreline. The detonation front was recorded at 300,000 fps and the velocity between each frame was measured according to Section 3.4.2 to determine if the velocity remained constant. A constant velocity indicates that the detonation does not fail, i.e., it propagates continuously. A velocity that is increasing and decreasing is consistent with a detonation that is failing and re-initiating. Position-time $(\mathrm{x}-\mathrm{t})$ diagrams were generated and a sample of one is presented for a $60 \mathrm{kPa}$ test in Figure 4.15. The $\mathrm{x}$-axis represents distance from the ignition end of the tube, and $\mathrm{y}$-axis displays time in seconds (each frame is 3.33 microseconds apart). The field-of-view considered spans five orifice plates (OP 5 - 9) and the $12.7 \mathrm{~mm}$ thick orifice plates appear as vertical black bands. The white data points represent the position measured and are overlaid on top of the video frame from which it was taken. The linear relationship indicates a constant velocity of $2760 \mathrm{~m} / \mathrm{s}$ along the centreline, with a standard deviation of $100 \mathrm{~m} / \mathrm{s}$. After the detonation front collides with the obstacle surface the reflected wave (leading edge of the bright white area to the right of the obstacle) can be observed propagating upstream. After the detonation wave passes roughly midspan between obstacles, the explosion fronts, originating at the tube wall, can be seen entering the narrow slit field-of-view transversely towards the centerline. 


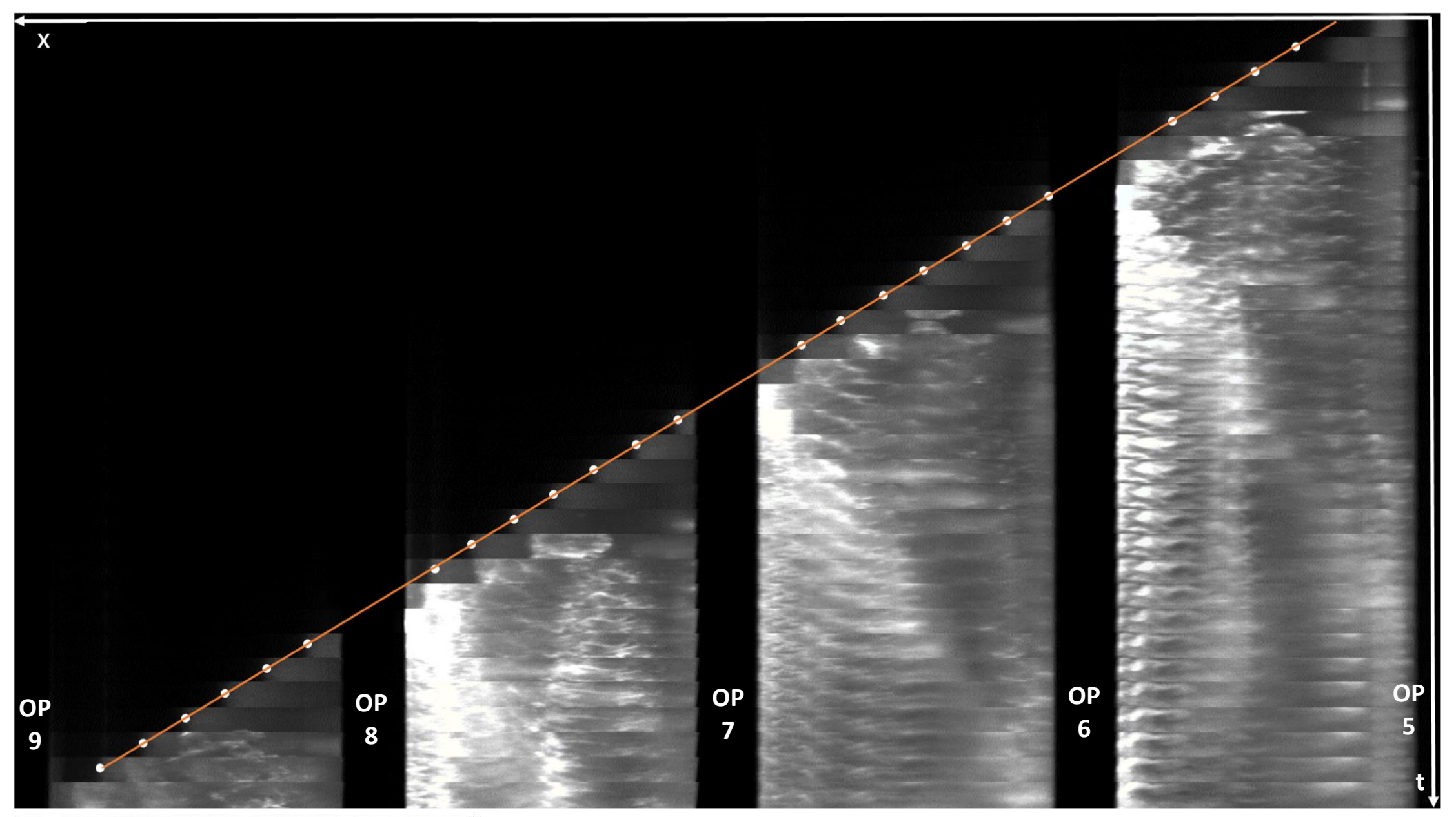

Figure 4.15: $x-t$ diagram generated showing constant velocity over a five obstacle field-of-view for a $60 \mathrm{kPa}$ test with $50 \% \mathrm{BR}$ (Test 137 ) 
For these very high initial pressure tests, when the decoupled shock and flame interact with the tube wall, the detonation is re-initiated at numerous sites. This is clearly seen in the image shown in Figure 4.16, where equally spaced detonation kernels appear in a ring around the inner-wall of the tube. Because of the perspective change over the camera field-of-view, the kernels at the back part of the wall can also be seen. It is believed that each kernel corresponds to the location where a triple-point from the failed detonation wave contacts the wall (shown in the circle in Figure 4.13), because the triple-point corresponds to the highest pressure and temperature along the shock front. In Figure 4.14, two kernels can be seen forming at the top and bottom of the tube (as indicated by the arrows in Image 3) and propagating forward with the combustion front (indicated by the arrows in Image 4).

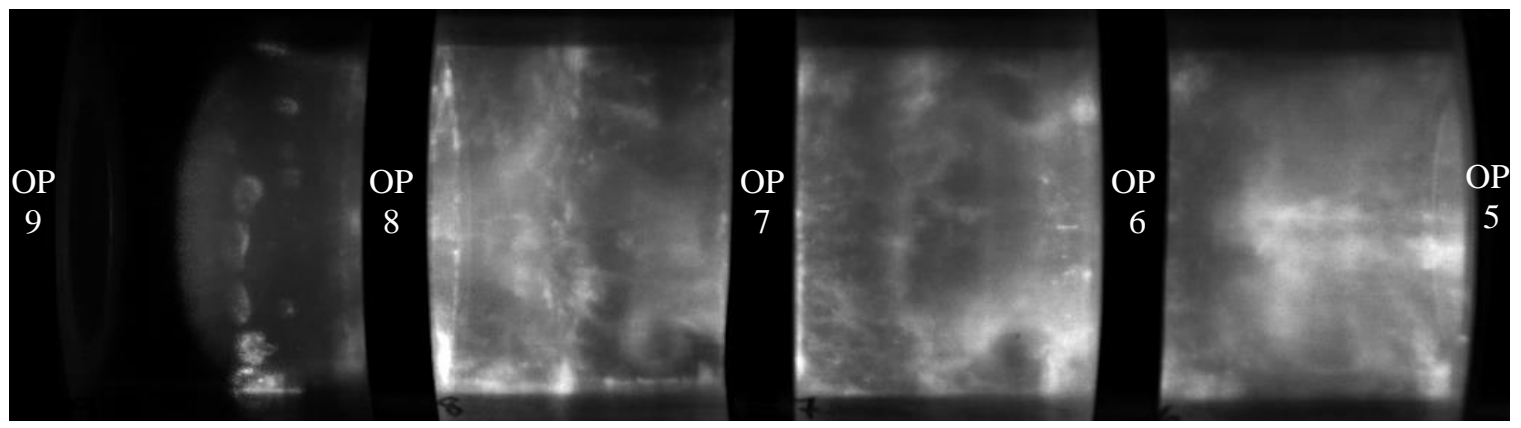

Figure 4.16: Still image from a $60 \mathrm{kPa}$ test with $50 \% \mathrm{BR}$, showing ring of detonation 'bubbles' forming on the tube wall, filmed at 70,000 fps (Test 139)

A soot foil placed on the tube wall from a similar $60 \mathrm{kPa}$ test is presented in Figure 4.17. A series of small dark 'lobes' are present around the entire foil at an axial location where the detonation kernels appear in the video. Fine cell structure is not visible in these dark lobes as would be expected if they were the result of detonation initiation. The vertical lines between the dark lobes (indicated by circles in Figure 4.17) occur where the detonation kernels collide with each other. Since the decoupled flame follows closely behind the lead shock wave there is very little compressed gas for the overdriven detonation wave to propagate through towards the center of the tube. The detonation waves can propagate downstream into the 
uncompressed unburned gas, but again it is difficult to see cells in the dark bottom half of the foil. These detonations, which would merge, do not influence the main detonation front that propagates along the centerline core.

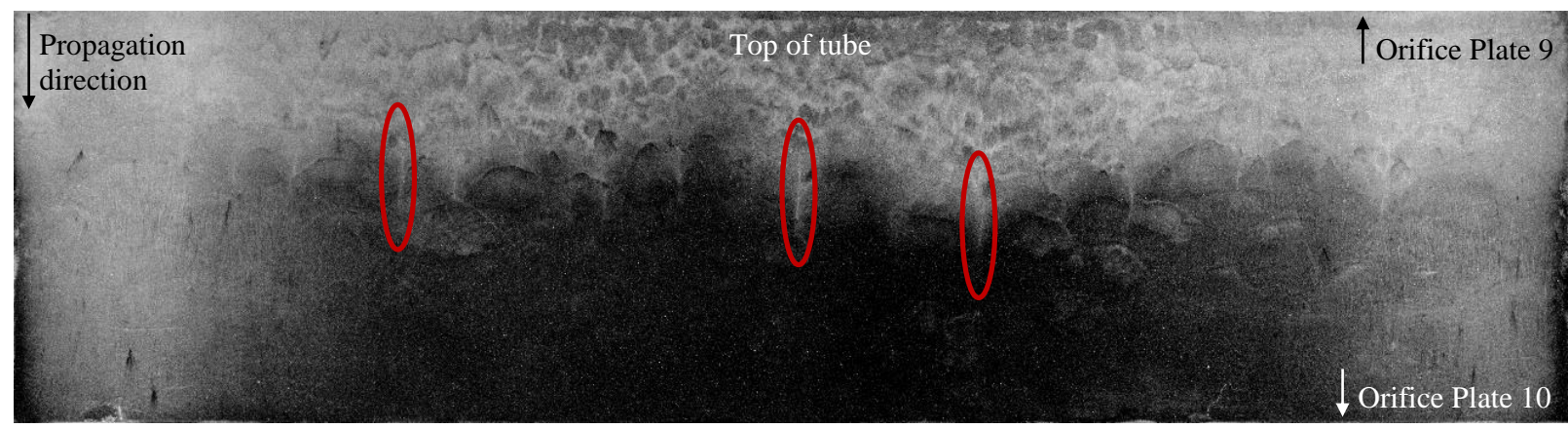

Figure 4.17: A curved soot foil for a $59.8 \mathrm{kPa}$ test showing detonation 'lobes' with 50\% BR (Test 157)

A schematic of the continuous detonation propagation mode is presented in Figure 4.18. The portion of the detonation wave along the channel centerline does not fail, while the outer portion decouples before collision with the tube wall. The red flashes represent the DDT kernels occurring in a ring on the tube wall far behind the centerline detonation front (as seen in Figure 4.16).

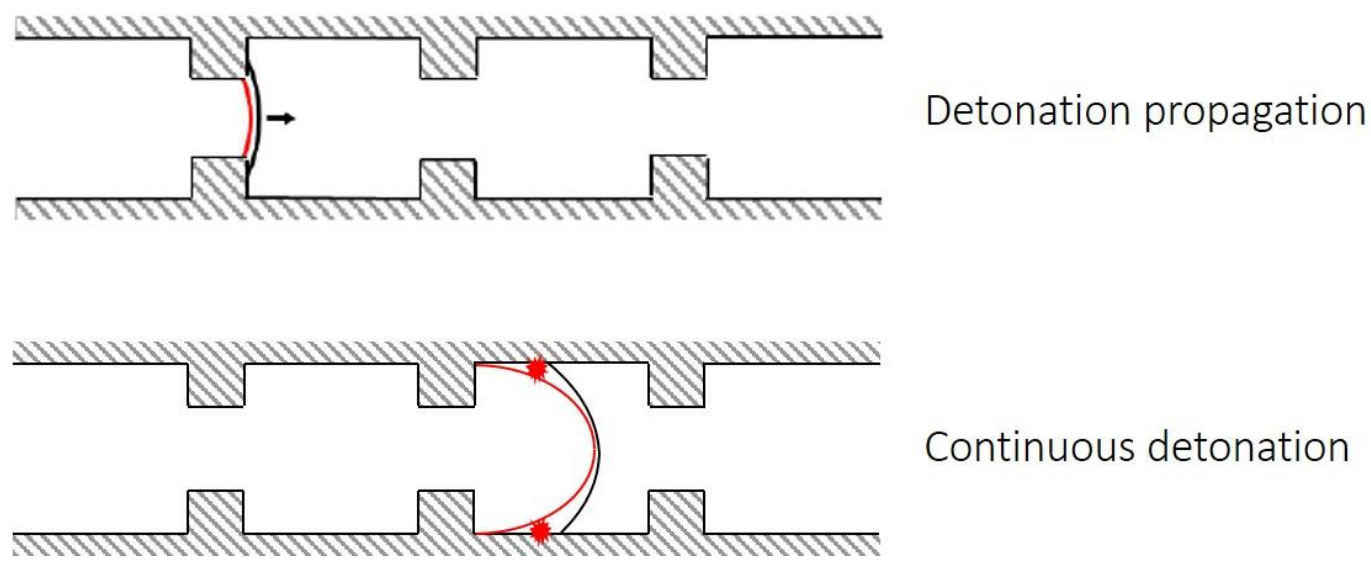

Figure 4.18: Schematic showing continuous detonation propagation mode 


\subsubsection{Obstacle Face Ignition Detonation}

A transitory mode was observed between the fast-flame regime and the single hot-spot wall ignition detonation regime. Preliminary results indicated that this might be a fourth mode of detonation propagation; however, extending the cylindrical channel demonstrated that this was not a sustainable detonation propagation mode.

Figure 4.19 shows frames from side-view video of a test performed at $8 \mathrm{kPa}$, spanning Orifice Plates 5 - 9. At $8 \mathrm{kPa}$, a fast-flame propagates in the last segment of the square channel and the wave continues to propagate as a fast-flame in the round channel up to OP 6. Between OP 5 and 6, the flame is barely visible until the reflected shock from the sixth orifice plate (see Image 3) interacts with the flame in Image 4. This is a fast-flame based on the definition that the reflection does not produce a local detonation wave. After OP 6 there is a transition in the propagation mechanism where a detonation is initiated at the obstacle face following shock reflection, producing the bright light on the obstacle face seen in Images 2 , 6 and 9. In Image 6, the faint detonation wave (shaped as a torus) is visible coming off the face of the eighth orifice plate.

Over the course of a few obstacles, the propagation mode transitions from igniting upon reflection with the obstacle face, to the single hot-spot wall ignition detonation propagation mode outlined in Section 4.1.3.2. The length of time to transition depends on the initial pressure of the test, where lower initial pressures take longer to transition. 

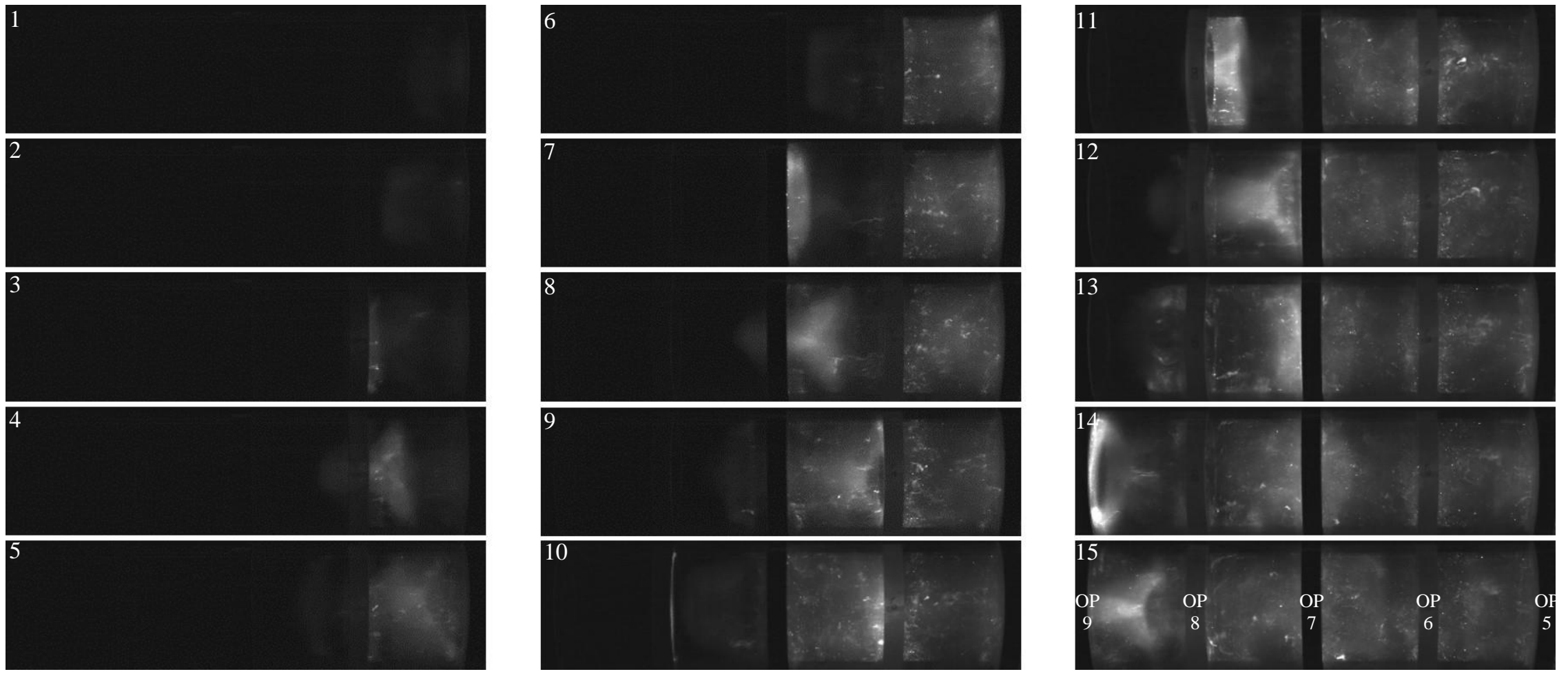

Figure 4.19: Images of combustion front propagating from right to left for an $8 \mathrm{kPa}$ test (Test 56), captured at 70,000 fps. The camera is centered on OP 7, as a result the right-face of obstacles 8 and 9 can be seen when illuminated by ignition caused by shock reflection as in image 6 and 9. The bright white tiny spots seen are burning particles of plastic eroded from the inside surface of the orifice plates. 
The soot foil record obtained on the tube wall between OP 9 and 10 for a similar $8 \mathrm{kPa}$ test is shown in Figure 4.20. The presence of very fine cells just before OP 10 is characteristic of a detonation initiated following shock reflection off the orifice plate face. The detonation propagates back towards OP 9 until it reaches the flame, at which point the cellular structure disappears. The absence of cells immediately after OP 9 indicates the detonation does not survive the diffraction after passing through the orifice plate. This behavior is similar to the detonation initiation on the obstacle face and subsequent failure observed in the rectangular channel for the same blockage ratio for initial pressures between 10 and $20 \mathrm{kPa}$ [8].

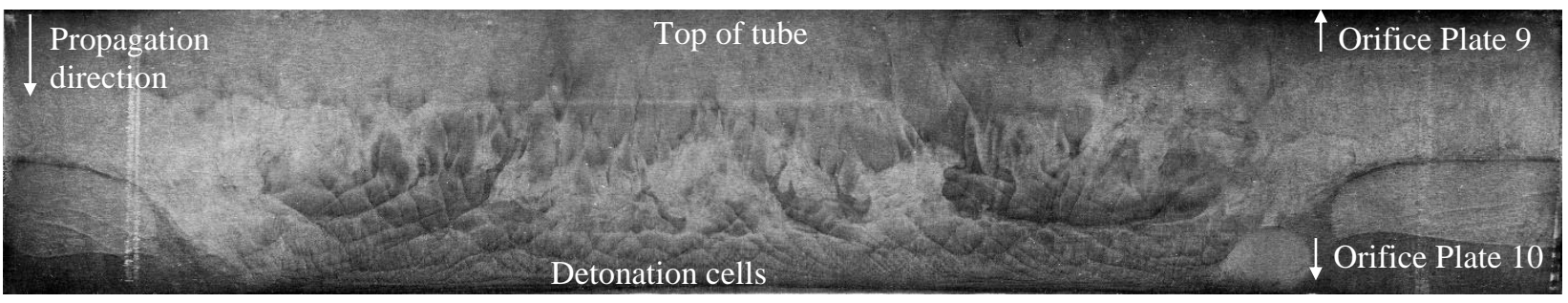

Figure 4.20: Curved soot foil obtained for an 8 kPa test with $50 \%$ BR (Test 89)

Figure 4.21 shows a schematic of the proposed mechanism for obstacle face ignition detonation. As the detonation progresses through the orifice plate, the detonation fails due to diffraction causing a decoupling of the leading shock (shown in black) and flame (shown in red) as seen in Figure 4.21b. As the leading shock reflects off the obstacle face, the temperature and pressure rise in the region of unburned gas between the leading shock and flame. The increased temperature and pressure cause detonation initiation to occur on the obstacle face. 
a)

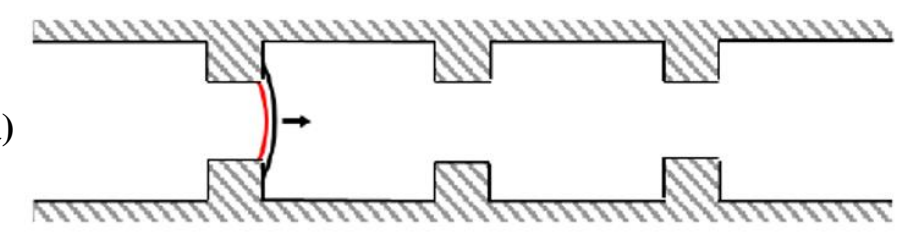

b)
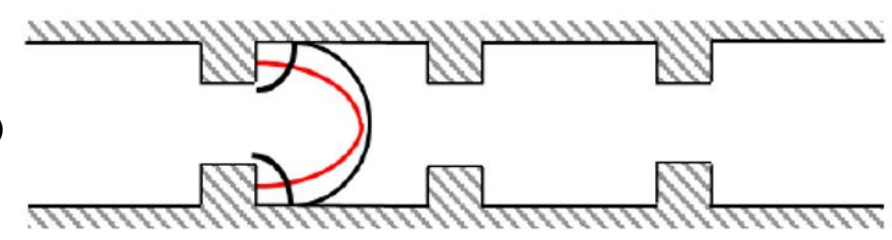

c)

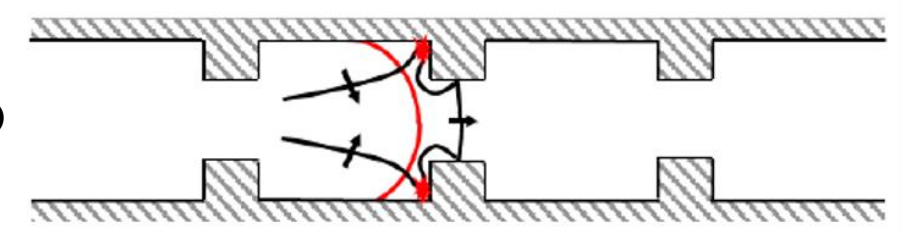

Detonation propagation

Detonation failure due to

diffraction

Detonation initiation off

obstacle face

Figure 4.21: Schematic showing obstacle face ignition detonation adapted from [43]

\subsection{5\% Blockage Ratio Results}

This section summarizes the results obtained using the $75 \%$ BR orifice plates in the cylindrical channel. These orifice plates have the same obstacle opening $(\mathrm{d}=3.81 \mathrm{~cm})$ as the fence-type obstacles in the square channel, but a higher blockage ratio $75 \%$ in the round tube compared to $50 \%$ in the square channel). Tests were performed at initial pressures between $4 \mathrm{kPa}$ and $60 \mathrm{kPa}$.

\subsubsection{Steady-State Tests}

A series of tests were conducted to verify that steady-state behaviour was observed in the preliminary (one-segment round tube) tests. Combustion wave propagation was observed down the length of the entire cylindrical channel and recorded at a speed of 300,000 fps. These videos were used to generate velocity profiles for each initial pressure, as outlined in Section 3.4.2, and to characterize changes in propagation modes, as seen in Section 4.2.3.6.

A summary plot of these velocity profiles is presented in Figure 4.22. For clarity, only a selection of results are shown. The speed of sound in the combustion products and the CJ detonation velocity were 
averaged over the pressure range and plotted for reference. The vertical dashed line on the plot indicates the end of the first segment of the cylindrical channel. Error bars are not shown on the plot for clarity, but each velocity has an associated error ranging from approximately $\pm 50 \mathrm{~m} / \mathrm{s}$ for the lower pressure tests $(4-$ $35 \mathrm{kPa}$ ) and up to approximately $\pm 250 \mathrm{~m} / \mathrm{s}$ for the very high pressure tests. The full associated error analysis is presented in Appendix B. The velocity point plotted at an obstacle number represents the average velocity between that $\mathrm{OP}$ and the previous OP (i.e. the data point at $\mathrm{OP} 10$ represents the average velocity between OP 9 and 10). The average velocity in the last segment of the square channel (measured according to Section 3.3.1) is plotted as a square marker at Obstacle 0 for each pressure.

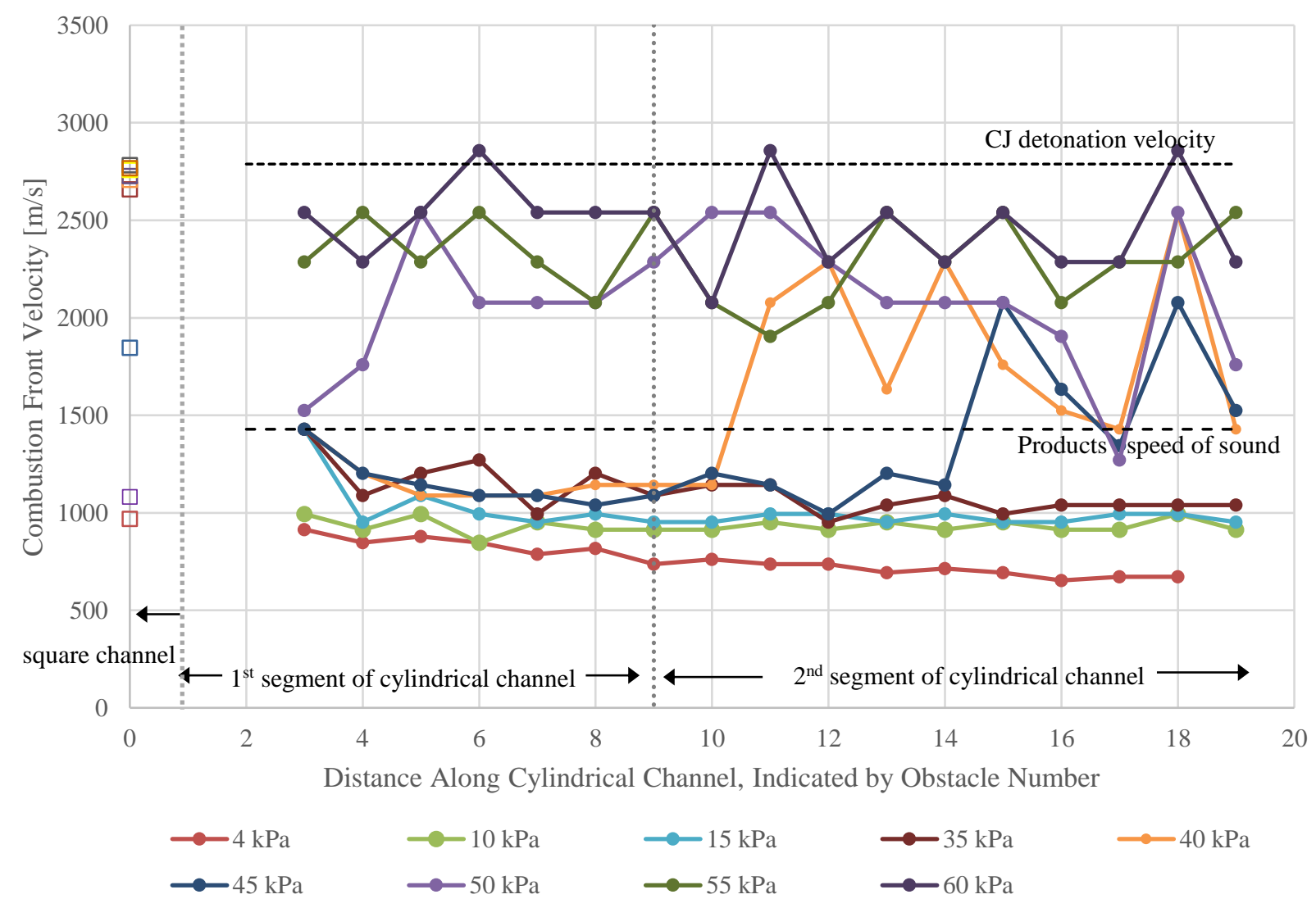

Figure 4.22: Plot of combustion front velocity profiles along the entire cylindrical channel for various initial pressures $(\mathbf{B R}=\mathbf{0 . 7 5})$ 
Based solely on the measured velocities presented in Figure 4.22, the combustion wave propagation in the round tube and square channel can be classified as follows.

- For initial pressures between 4 and $10 \mathrm{kPa}$, a fast-flame emerged from the square channel and continued to propagate through the cylindrical channel as a fast-flame, i.e., the flame propagated below the speed of sound at a steady velocity of approximately $1000 \mathrm{~m} / \mathrm{s}$. For this pressure range, the preliminary tests are representative of steady-state behaviour.

- At initial pressures between $12 \mathrm{kPa}$ (not shown) and $35 \mathrm{kPa}$, a detonation emerged from the square channel, but quickly transitioned to a fast-flame (within 2 or 3 obstacles) which continued to propagate through the cylindrical channel. For this pressure range, the preliminary tests produced a steady propagating wave.

- For initial pressures between 40 and $45 \mathrm{kPa}$, a detonation emerged from the square channel and quickly transitioned to a fast-flame. The fast-flame propagated down the length of the first half of the channel and partly through the second where DDT occurred. For this range of pressures, the preliminary tests are not representative of steady-state behaviour as the shorter channel only captured the initial fast-flame propagation.

- For initial pressures between 50 and $60 \mathrm{kPa}$, a quasi-detonation was transmitted from the square channel and continued to propagate through the round tube. As quasi-detonation behaviour was immediately observed in the round tube, the preliminary tests produced steady state propagation for this pressure range.

In summary, these tests demonstrated that experiments completed in the 1-segment channel at initial pressures between $40 \mathrm{kPa}$ and $50 \mathrm{kPa}$ do not provide an accurate representation of the steady-state propagation mode.

\subsubsection{Comparison of Cylindrical and Square Channel Combustion Front Velocities}

Figure 4.23 presents a plot of the average combustion front velocity as a function of initial pressure in the square $(\mathrm{BR}=0.5)$ and cylindrical channels $(\mathrm{BR}=0.75)$. The average combustion front velocity in the 
square and cylindrical channels was determined according to Sections 3.3.1 and 3.4.2, respectively. For each test, there are two velocities plotted: one in the square channel and one in the round channel. For transparency, the round channel tests completed in the two-segment cylindrical channel and the steady onesegment cylindrical test results (less than $35 \mathrm{kPa}$ and greater than $50 \mathrm{kPa}$ ) are plotted separately.

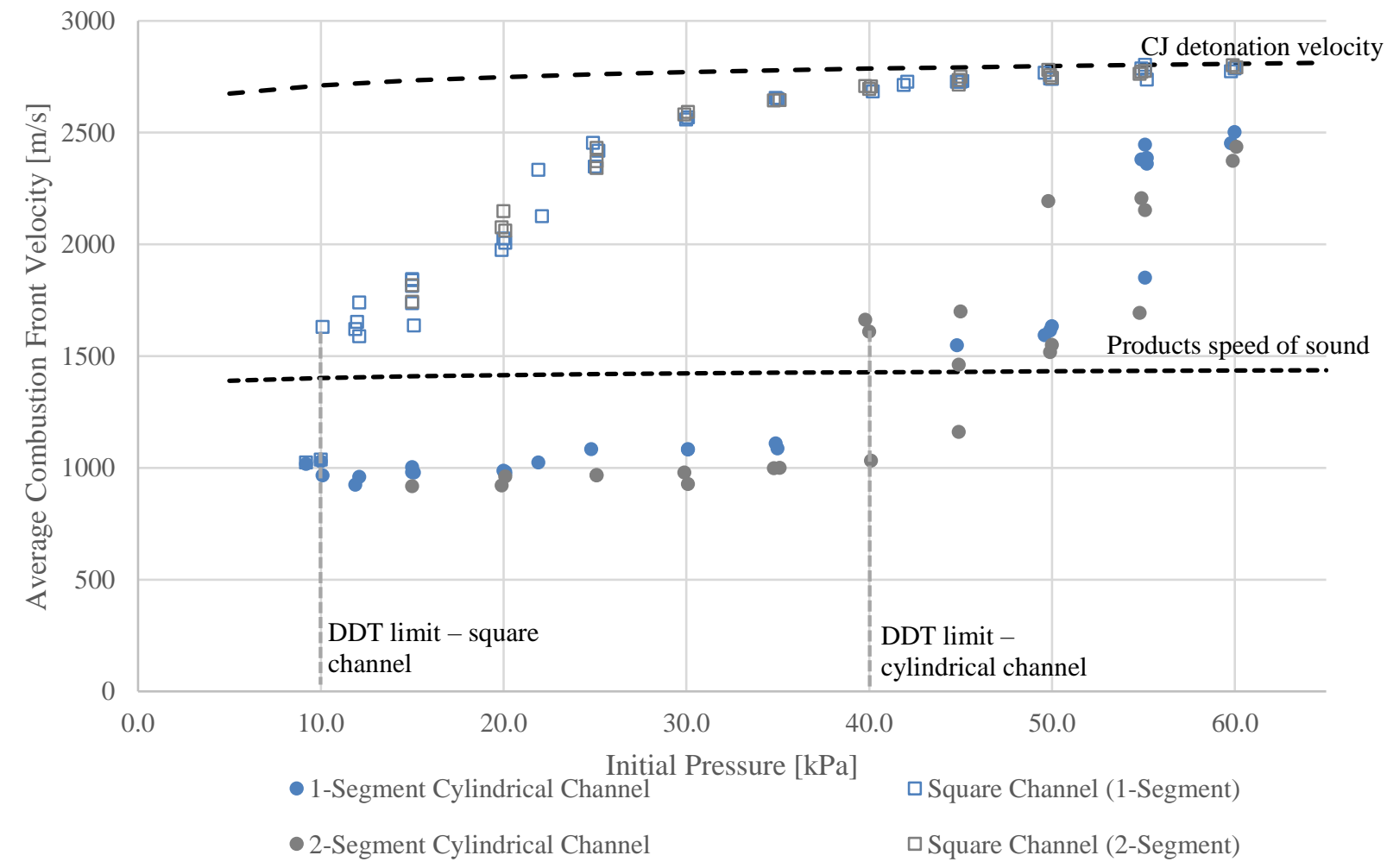

Figure 4.23: Plot of average combustion front velocity as a function of initial pressure for $75 \% \mathrm{BR}$ in the cylindrical channel and $50 \% \mathrm{BR}$ in the square channel

According to the classical definition of the detonation limit (an average propagation velocity greater than the products speed of sound), the limit is $40 \mathrm{kPa}$ in the cylindrical channel and is $10 \mathrm{kPa}$ in the square channel. These limits are indicated by vertical dashed lines in Figure 4.23. As the cylindrical and square channels have the same opening $(d=3.81 \mathrm{~cm})$, they both have the same $d / \lambda$ value for any given initial pressure. The detonation limit in the square channel $(10 \mathrm{kPa})$ corresponds to a $\mathrm{d} / \lambda$ of 1.6 (the same as in the 
$50 \%$ BR tests reported in Section 4.1.2), but the detonation limit in the cylindrical channel (40 $\mathrm{kPa})$ corresponds to a $\mathrm{d} / \lambda$ of 14.3 . The detonation limit in the cylindrical channel is significantly larger than Peraldi's $d / \lambda \geq 1$ criterion [5]. Although it was shown in [11] that the critical $d / \lambda$ ratio for DDT increased with blockage ratio, the detonation limit found experimentally exceeded the $d_{d} / \lambda \approx 13$ criterion for the critical tube diameter.

For tests $\left(40 \leq \mathrm{P}_{\mathrm{i}} \leq 55 \mathrm{kPa}\right)$ that generated quasi-detonations (combustion waves propagating between the $\mathrm{CJ}$ detonation velocity and the products speed of sound), there are large test-to-test variations and the measured average velocities fall well below the CJ detonation value. Comparably, at $60 \mathrm{kPa}$, the test-to-test variation and the $\mathrm{CJ}$ detonation velocity deficit are small. A higher propagation velocity was observed in the last segment of the square channel than in the cylindrical channel. As in the $50 \%$ BR tests, this is likely due to 2-D diffraction around obstacles in the square channel and 3-D diffraction through the orifice plates in the cylindrical channel.

The fast-flame propagation velocity was comparable in both channels (over the pressure range of 9 and $10 \mathrm{kPa}$ ) which is consistent with the results from the $50 \%$ BR tests. This can be attributed to the fact that fast flame propagation is unaffected by diffraction, and is governed by shock-flame interactions that develop from shock reflections, as shown in [8].

\subsubsection{Propagation Modes in the Cylindrical Channel}

The video footage was used, in conjunction with soot foils for some tests, to observe and classify combustion wave propagation in the cylindrical channel. At initial pressures less than $35 \mathrm{kPa}$, a fast-flame was observed. Unlike in the $50 \%$ BR tests, there was a high degree of variability in quasi-detonation propagation modes occurring at a given initial pressure and even within the same test. There were three quasi-detonation propagation modes observed: obstacle face ignition detonation $\left(\mathrm{P}_{\mathrm{i}} \geq 35 \mathrm{kPa}\right)$, single hotspot wall ignition detonation $\left(\mathrm{P}_{\mathrm{i}} \geq 40 \mathrm{kPa}\right)$, and multi hot-spot wall ignition detonation (the dominant mode for $50 \mathrm{kPa} \leq \mathrm{P}_{\mathrm{i}} \leq 60 \mathrm{kPa}$ ). A fourth interesting phenomenon was observed for initial pressures between 20 and $35 \mathrm{kPa}$, where ignition occurred at local hot-spots which formed far behind the flame front and lead 
shock. The continuous detonation mode was not observed, as pressures greater than $60 \mathrm{kPa}$ were not tested due to the pressure constraints of the round tube. Due to the variability in wave propagation mode down the length of the tube, the soot foil method was not used for pressures below $50 \mathrm{kPa}$.

\subsubsection{Fast-Flame Regime}

A propagating fast-flame was observed for initial pressures between $4 \mathrm{kPa}$ and $35 \mathrm{kPa}$, where 35 $\mathrm{kPa}$ corresponds to a $\mathrm{d} / \lambda$ of 10 in the cylindrical channel. The fast-flame regime is characterized by the absence of DDT and an average combustion front velocity in the $\sim 1000 \mathrm{~m} / \mathrm{s}$ range. Experimentally, the average flame propagation velocity ranged from $760 \mathrm{~m} / \mathrm{s}$ to $1090 \mathrm{~m} / \mathrm{s}$ depending on initial pressure, with the highest average propagation velocity occurring at $35 \mathrm{kPa}$.

Figure 4.24 shows a video sequence of a $15 \mathrm{kPa}$ test, recorded at 70,000 fps. In Image 1, the fastflame enters the field-of-view, emerging from the fourteenth orifice plate. The flame is faint between orifice plates, (as seen in Images 2, 3, 6, 7, 8, 11, 12, 13, 16, 17 and 18) but becomes brighter when the lead shock (not visible) reflects off the upstream face of the orifice plate. A detonation is not initiated at any point in the video. The fast-flame regime observed for the $75 \%$ BR tests is very similar to the one observed in the $50 \%$ BR tests and reported in [8]. The characteristic bright spots formed by the implosion of the reflected shock observed in [4] and in the 50\% BR tests are visible in the $75 \%$ BR tests. 

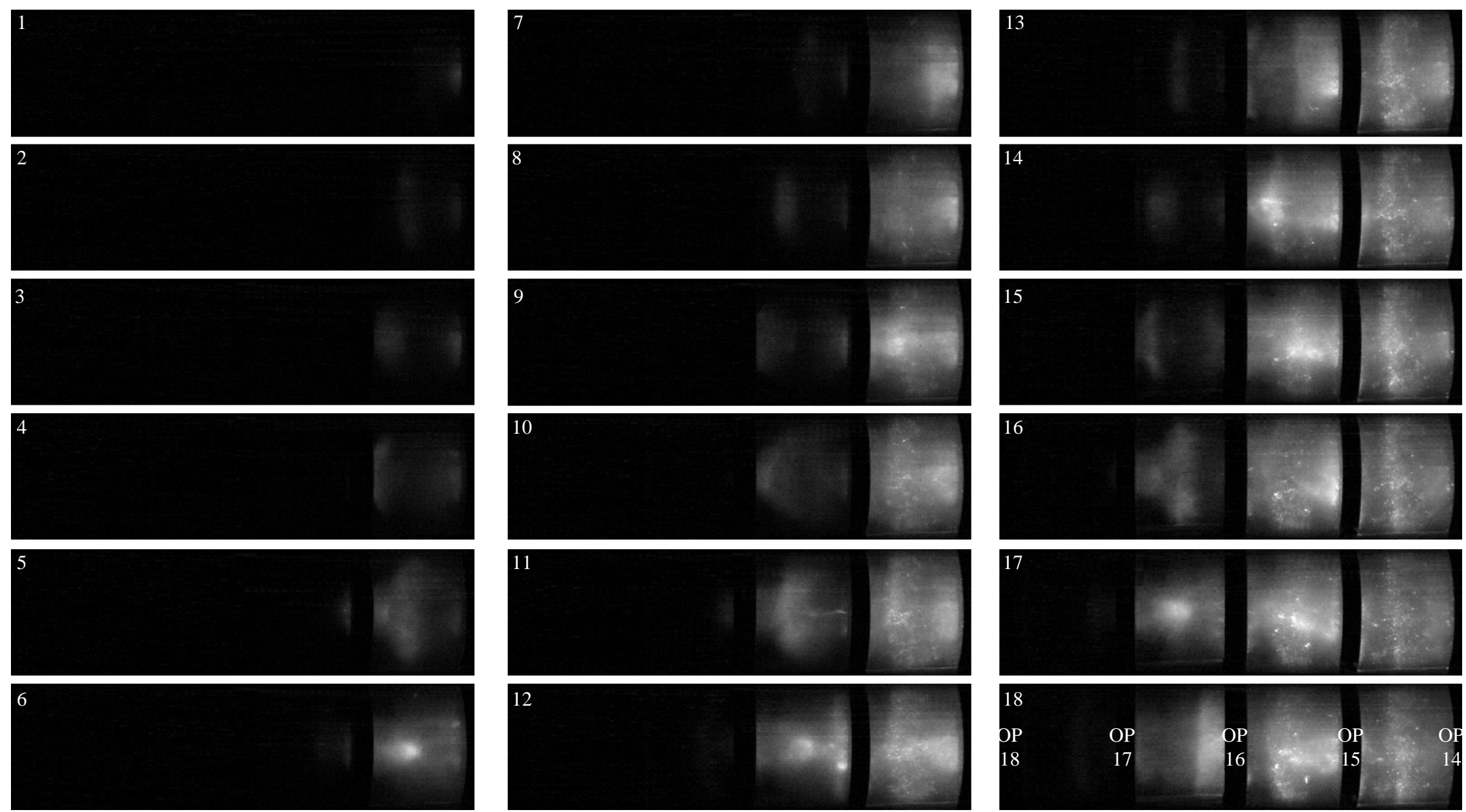

Figure 4.24: Side-view footage filmed at 70,000 fps for a $15 \mathrm{kPa}$ test with $75 \%$ BR showing fast-flame propagation (Test 270) 


\subsubsection{Obstacle Face Ignition Detonation}

The obstacle face ignition detonation mode, characterized by detonations initiated at the obstacle face, was the dominant mode in the lower pressure tests. This pressure range (35-40 kPa) corresponds to a d/ $\lambda$ of 10 to 14 , although detonations were initiated at the obstacle face at test pressures of up to $55 \mathrm{kPa}$. Figure 4.25 shows video captured at 70,000 fps during a $40 \mathrm{kPa}$ test. The obstacle face is illuminated when the leading shock collides with the orifice plate and a detonation is initiated (as seen in Images 3, 6 and 12). The detonation fails due to diffraction as it passes through the orifice plate and a fast-flame propagates to the next orifice plate. Detonation failure due to diffraction occurs at each orifice plate in the field-of-view except between OP 16 and OP 17 where the interaction of the fast-flame with OP 16 produces a detonation wave that successfully propagates to OP 17 . This was established based on the highly visible and quasiplanar wave observed in Image 8, and the high propagation velocity of approximately $2530 \mathrm{~m} / \mathrm{s}$. This video highlights the variation in propagation modes for the $75 \%$ BR orifice plates. The detonation wave initiated at an obstacle face sometimes survived diffraction through the orifice plate and continued as a detonation to the next orifice plate - a behaviour which was also observed in a square channel [8]. Parameters affecting this are the strength and angle of the shock wave reflecting off the orifice plate.

The average combustion front velocity for this test, taken over the field-of-view between OP 14 18 , is approximately $1030 \mathrm{~m} / \mathrm{s}$. This velocity is below the products speed of sound for a stoichiometric hydrogen-oxygen mixture at an initial pressure of $40 \mathrm{kPa}$, which is approximately $1425 \mathrm{~m} / \mathrm{s}$. A characteristic of obstacle face ignition detonations is that this propagation mode does not increase the average combustion front velocity above the speed of sound. According to the conventional detonation limit definition, this mode would not be classified as a quasi-detonation, although detonation initiation and propagation did occur. Based on the velocity data in Figure 4.23, $40 \mathrm{kPa}$ corresponds to the detonation limit, where other tests at this pressure produced quasi-detonations. 

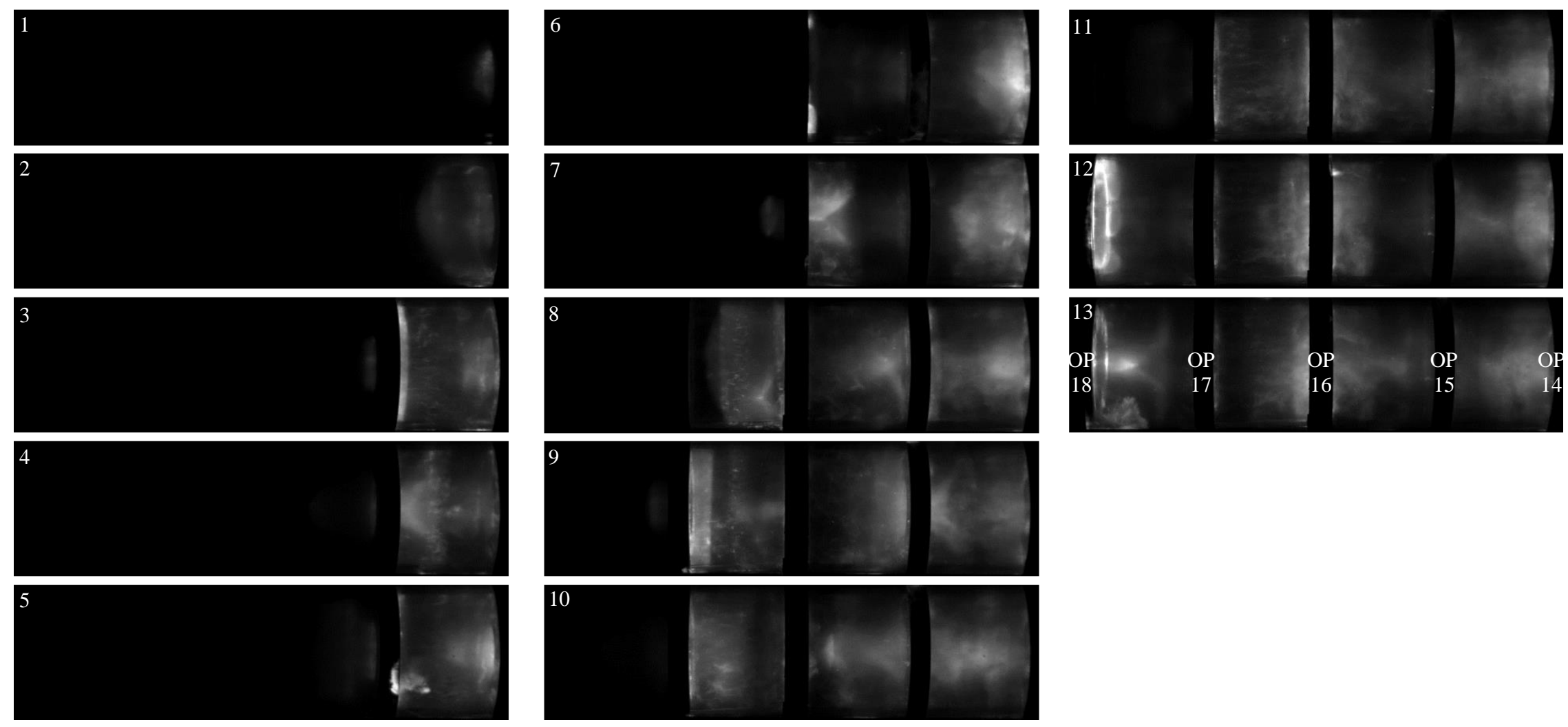

Figure 4.25: Side-view video captured at 70,000 fps for a $40 \mathrm{kPa}$ test with $75 \%$ BR showing obstacle face ignition (Test 247) 
The obstacle face ignition mode is shown at a higher framing rate of 300,000 fps for a $45 \mathrm{kPa}$ test in Figure 4.26. A detonation is initiated at a single point on the orifice plate at the bottom of the tube (as indicated by the arrow in Image 3). The two fronts of the detonation (shown by the arrows in Images 4 and 5) subsequently spread around the orifice plate until they meet (shown in Images 4 - 13). The wave travels half the circumference of the inner-diameter of the tube (considering that it is propagating in two directions). By dividing this distance by the time elapsed, it was determined that the wave was travelling at approximately $3270 \mathrm{~m} / \mathrm{s}$, confirming that it was an overdriven detonation (the $\mathrm{CJ}$ detonation velocity at this initial pressure is $2790 \mathrm{~m} / \mathrm{s}$ ). This velocity is greater than the CJ detonation velocity because the region of unburned gas it is travelling through is at an elevated temperature and pressure.
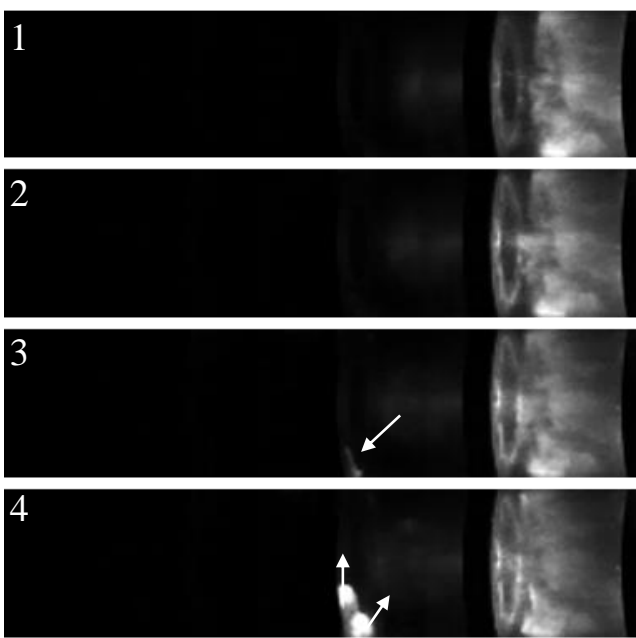

5

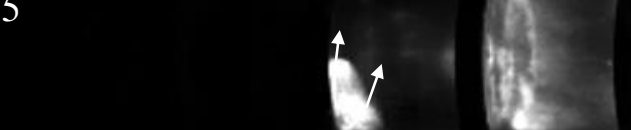

6
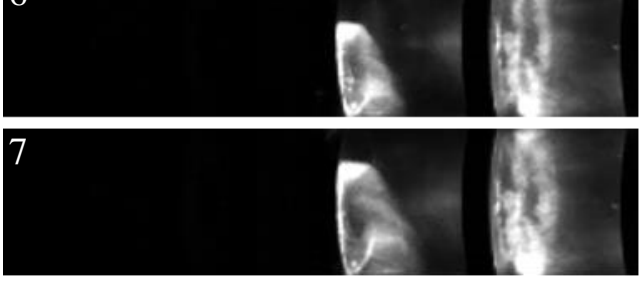
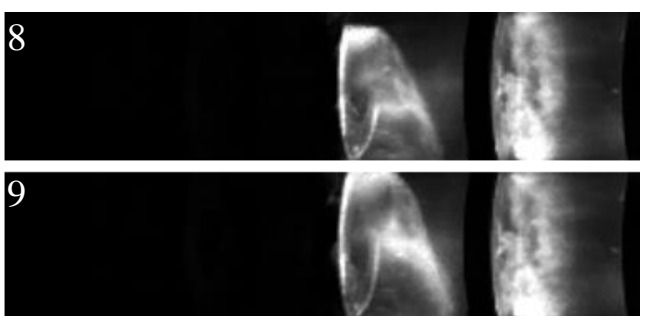

10
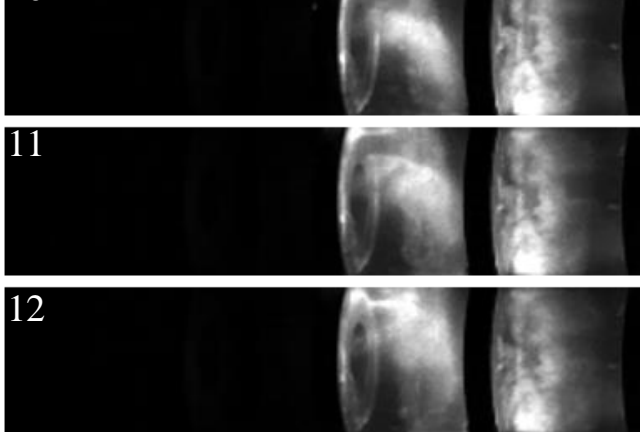

13
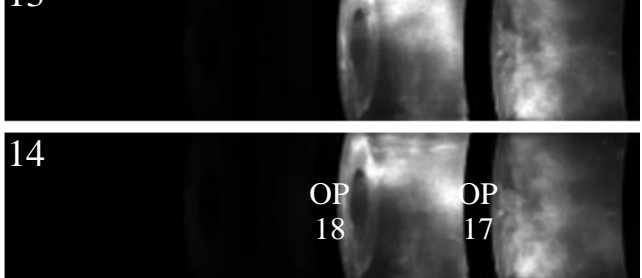

Figure 4.26: Obstacle face ignition initiated at a single point for a $45 \mathrm{kPa}$ test with $75 \% \mathrm{BR}$ filmed at 300,000 fps (Test 242) 
A soot foil was used to confirm that the bright light generated at the orifice plate is associated with detonation initiation following shock reflection. A soot foil obtained from a $50 \mathrm{kPa}$ test, shown in Figure 4.27, shows that ignition occurs off the orifice plate face. The presence of detonation cells, a close-up of which is presented in the bottom image of Figure 4.27, immediately before Orifice Plate 10 indicates that the detonation was initiated following reflection of the lead shock on the obstacle face. It is believed that the marks at the top of the foil are due to condensation accumulating in the tube after the test was run.

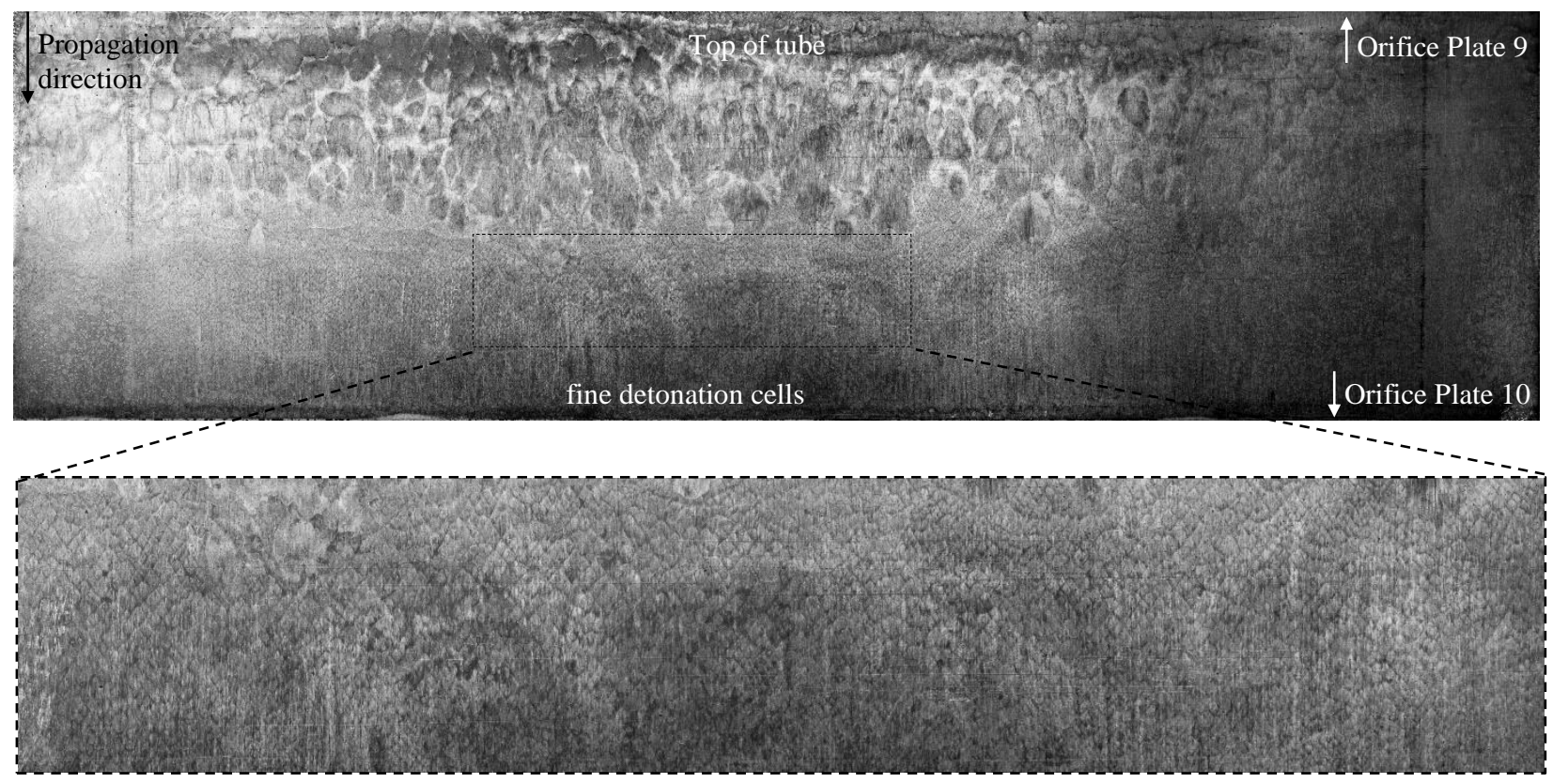

Figure 4.27: Top: Curved foil obtained for a $50 \mathrm{kPa}$ test with $75 \%$ BR installed between OP 9 and 10 showing detonation initiation off the obstacle (Test 111) Bottom: Close-up of the foil showing the very fine detonation cell structure

This detonation initiation mode is similar to the transitory obstacle face detonation initiation mode seen in the $50 \%$ BR tests, except that at $75 \%$ BR it was shown to be a sustainable mode. Shock reflection on the obstacle face generated a high reflected shock temperature and pressure and a local explosion is generated at the obstacle face. 
Just as detonations are initiated at a single site or multiple sites on the tube wall, detonation initiation also occurred at multiple sites on the orifice plate. Figure 4.28 shows detonations being initiated in at least three points on OP 17 for a $55 \mathrm{kPa}$ test, filmed at 300,000 fps. The detonations, shown forming in Image 3, expand and propagate backwards towards OP 16 in the region of heated, unburned gas between the lead shock and flame. As detonations grow radially, the three detonations simultaneously spread around the orifice plate and merge, eventually illuminating the entire face of the orifice plate in Image 5. The detonation initiation observed in Images 3 and 12 in Figure 4.25 (recorded at 70,000 fps) likely started at multiple sites.
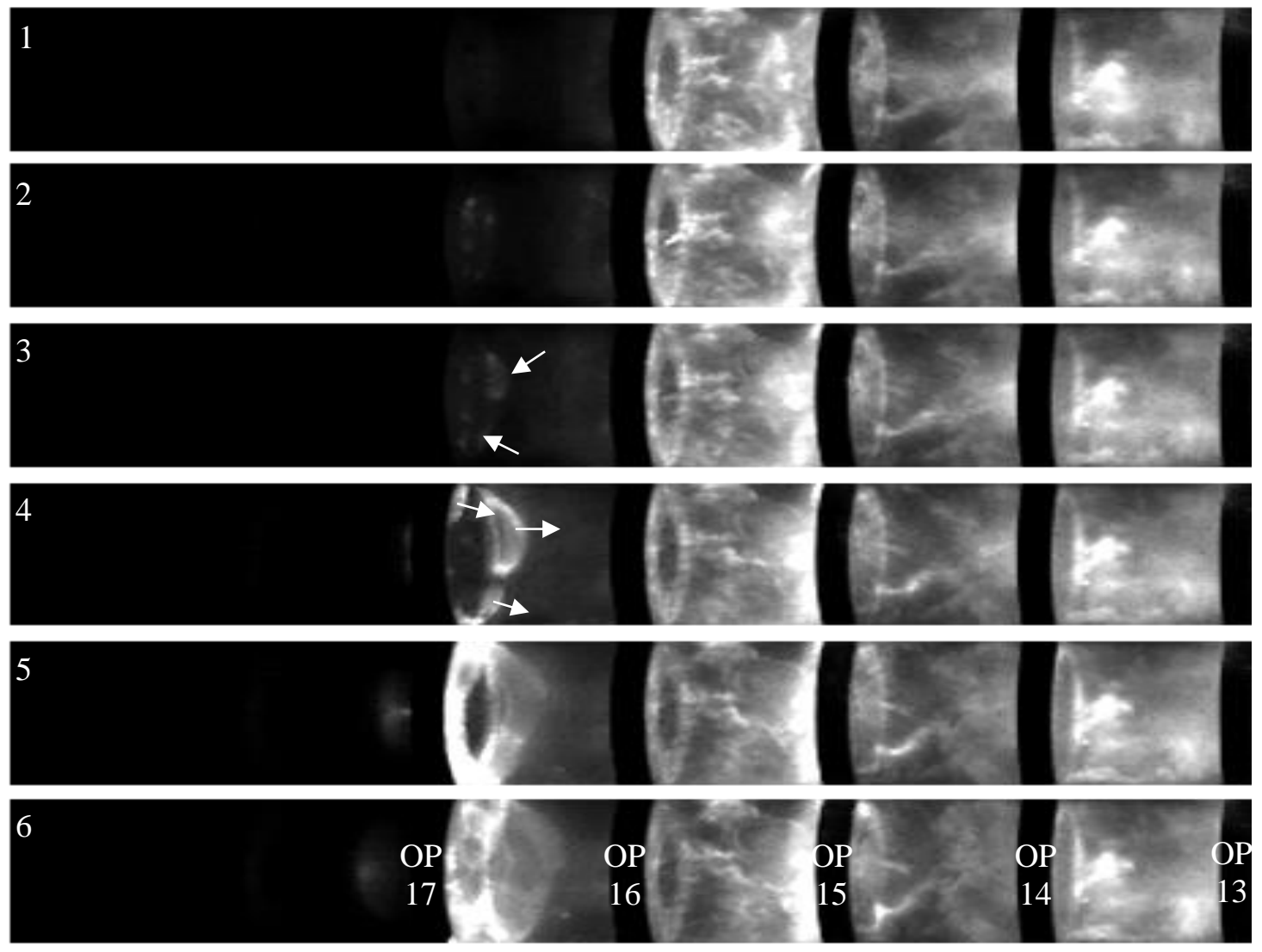

Figure 4.28: Video captured for a $55 \mathrm{kPa}$ test with $75 \%$ BR showing detonation being initiated at multiple sites on the obstacle face (Test 243) 
A close-up of detonation formation on the orifice plate is presented in Figure 4.29, a sequence of images filmed at 175,000 fps for a $50 \mathrm{kPa}$ test. The images show a detonation kernel forming on the orifice plate and spreading backwards in the unburned gas in the opposite direction of overall detonation propagation. The bright light on the inner edge of the orifice plate shown in Image 5, indicates when the lead shock first collided with the orifice plate. In Image 6, a detonation kernel (indicated by an arrow) has formed and grows, as shown in Image 7. The detonation kernel spreads back into the unburned gas towards Orifice Plate 7.
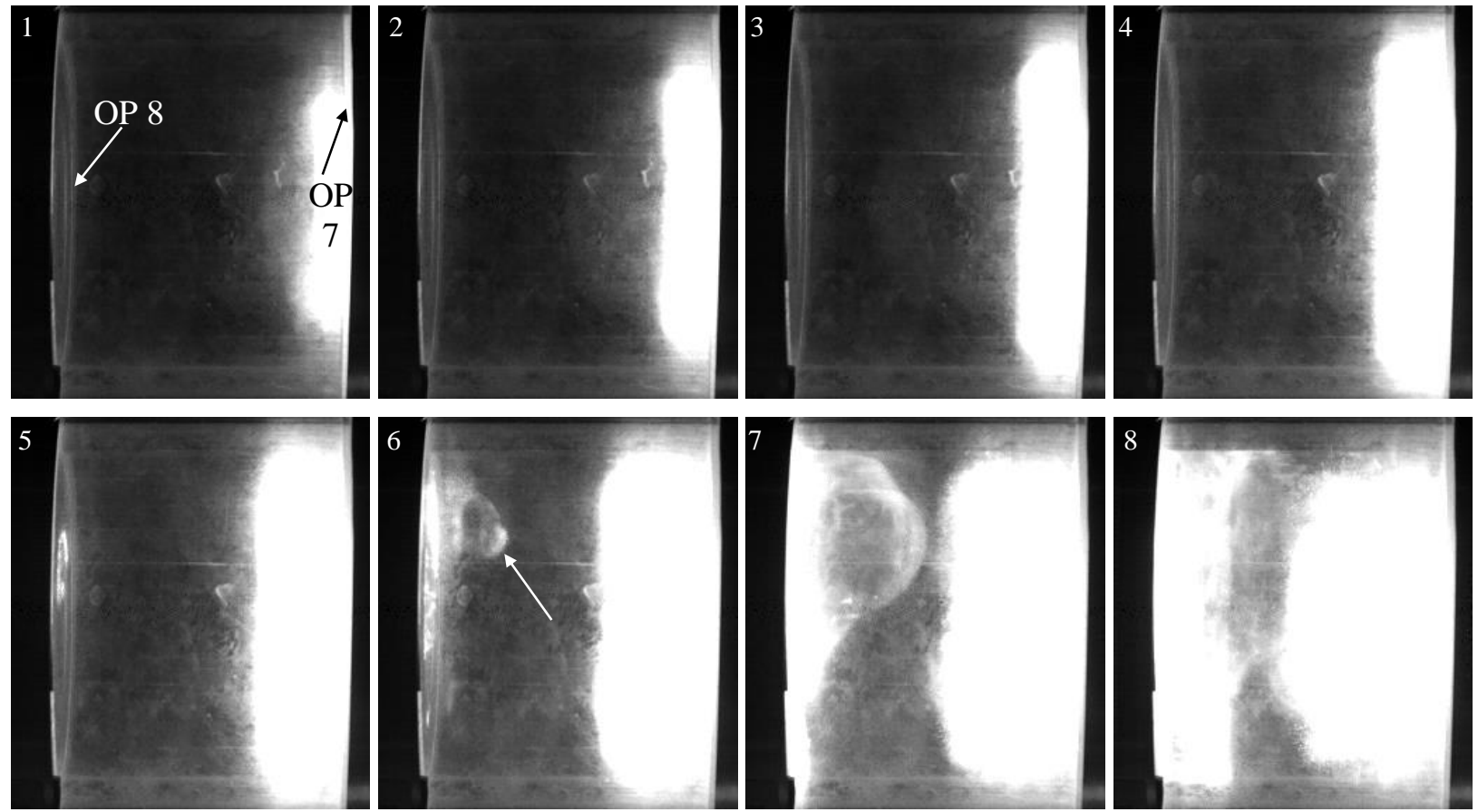

Figure 4.29: Side-view footage filmed at 175,000 fps for a $50.1 \mathrm{kPa}$ test with $75 \% \mathrm{BR}$ (Test 115) showing a detonation forming on OP 8

\subsubsection{Single Hot-Spot Wall Ignition Detonation}

As observed in $50 \%$ BR tests, in the $75 \%$ BR configuration detonations were initiated at a single hot-spot on the tube wall. This mode was observed for initial pressures of $40 \mathrm{kPa}$ and higher, where $40 \mathrm{kPa}$ corresponds to a $\mathrm{d} / \lambda=14$. This mode is shown for a $50 \mathrm{kPa}$ test in Figure 4.30, filmed at 175,000 fps. In 
Image 4, a single detonation kernel (indicated by the arrow) forms on the tube wall near the bottom of the tube. The detonation kernel grows radially, as indicated by the arrows in Image 5. As in the 50\% BR tests, the detonation wave collides with the bottom of the orifice plate first, (indicated by the portion of the orifice plates illuminated in Image 6). The two parts of the detonation front spread around the orifice plate face, shown in Image 7, and meet at the top of the orifice plate forming a very bright spot in Image 8 .
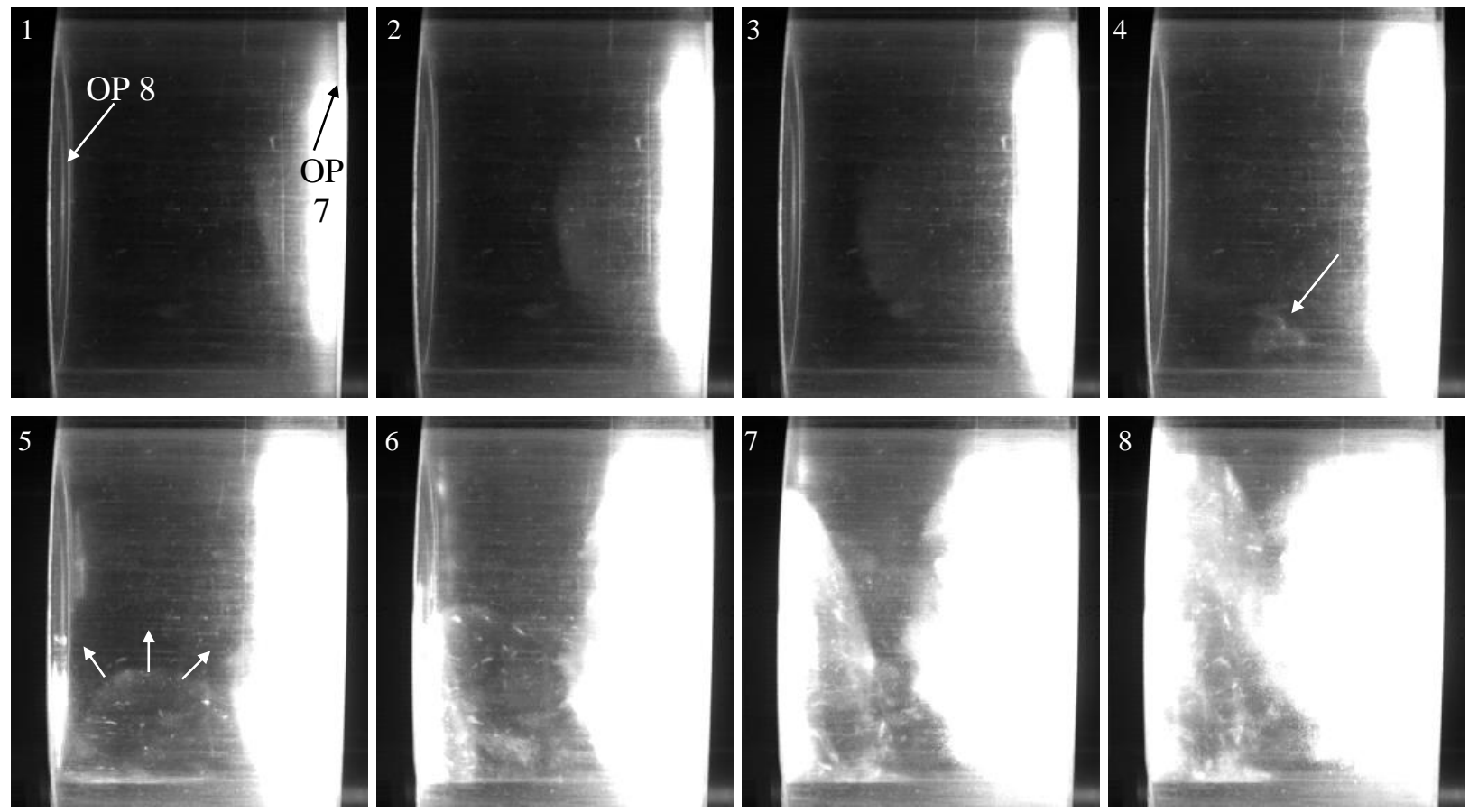

Figure 4.30: Side-view video footage captured between OP and for a $50 \mathrm{kPa}$ test with $75 \% \mathrm{BR}$ showing a single point of detonation initiation on the tube wall (Test 117)

A soot foil corresponding to a similar $50 \mathrm{kPa}$ test is presented in Figure 4.31. The curved foil was installed between Orifice Plates 9 and 10. The absence of cells immediately following OP 9 indicates that the detonation failed due to diffraction around the orifice plate. The detonation was initiated at a single point on the wall, indicated by an arrow, and spread in a triangular pattern towards Orifice Plate 10. The triangular pattern is clearly filled with detonation cells on the physical foil, although they are difficult to see in the photograph. This is a similar behavior to that observed in [6] and observed for the single-hot spot wall ignition mode seen at the $50 \%$ blockage ratio. However, unlike in the $50 \%$ blockage ratio tests or the 
experiments conducted in [6], there is also a band of detonation cells that run around the outside of the peak (labelled detonation cells on the image) and immediately before Orifice Plate 10. This suggests that another detonation may also have been initiated upon reflection from the orifice plates.

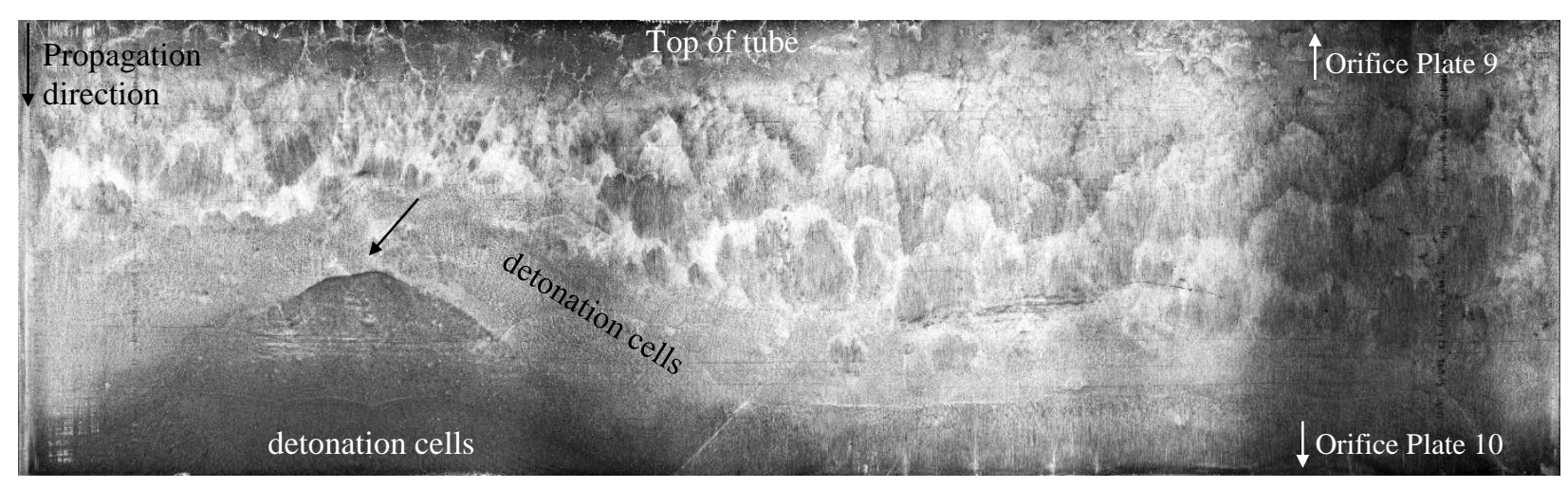

Figure 4.31: Curved soot foil obtained for a $50 \mathrm{kPa}$ test with $75 \%$ BR installed between Orifice Plates 9 and 10 showing detonation initiation from a single point on the wall (Test 106)

Interestingly, the slapping wave pattern of alternating ignition points was not observed for the $75 \%$ blockage ratio tests. One explanation for this is that since there are several different detonation initiation modes occurring for the same test it is difficult for the wave to lock into a single mode.

\subsubsection{Multi Hot-Spot Wall Ignition Detonation}

At very high initial pressures $(50-60 \mathrm{kPa})$, detonation initiation occurred at multiple hot-spots on the tube wall. This high pressure range corresponded to a d/ $\lambda$ of 18 at $50 \mathrm{kPa}$ and to 27 at $60 \mathrm{kPa}$. The video filmed at 70,000 fps for a $55 \mathrm{kPa}$ test is shown in Figure 4.32. Detonations were initiated after every orifice plate as seen in Images 3, 5 and 7; however, there are an insufficient number of frames to resolve the exact site of each detonation initiation. In Image 9, as indicated by the white arrows, detonations are initiated at two points at the top and bottom of the tube between OP 8 and 9. The image is over-exposed as the neutral density filter was not used for this particular test. 

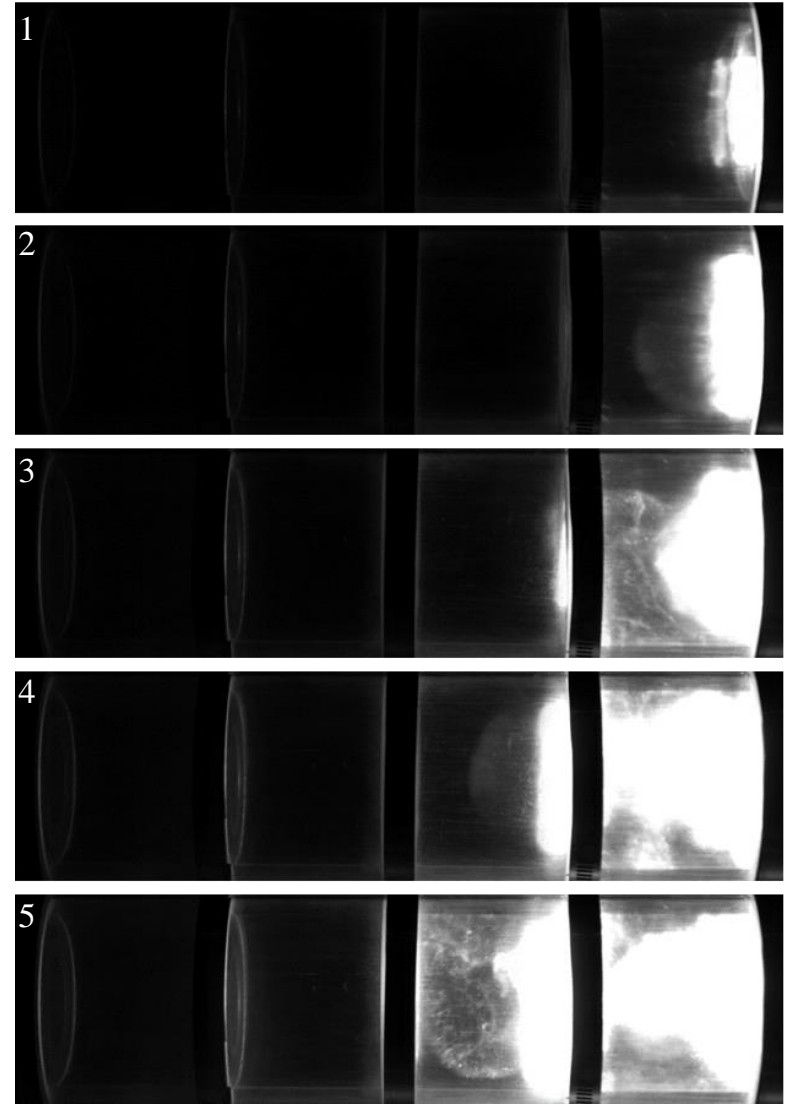
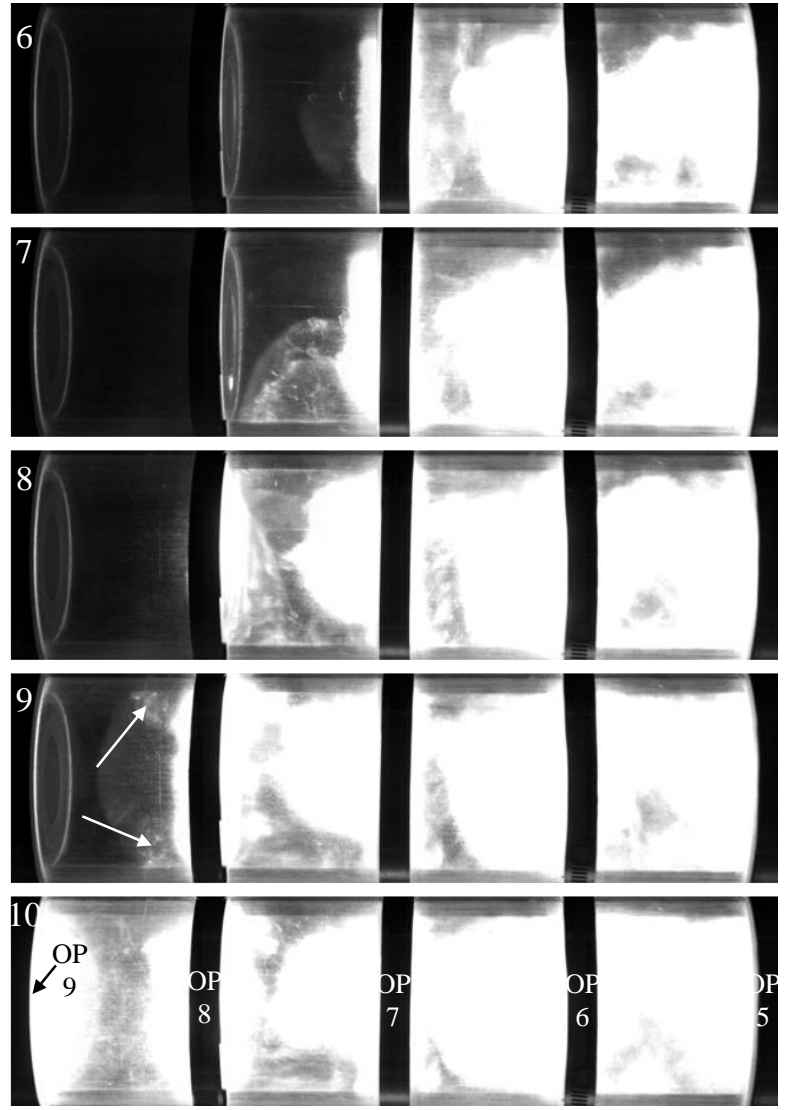

Figure 4.32: Side-view video of a $55 \mathrm{kPa}$ test with $75 \%$ BR showing multiple ignition points on the wall, filmed at 70,000 fps (Test 114)

Video captured at a higher frame rate $(300,000 \mathrm{fps})$ for another $55 \mathrm{kPa}$ test is shown in Figure 4.33. Between OP 12 and 13 detonations are initiated at two distinct hot-spots as indicated by the white arrows in Image 9. The detonations initiated at these hot-spots merge to form a single detonation in Image 10. In Image 8 and 9 the combustion front can be clearly seen ahead of the detonations, but by Image 11 the detonation has overtaken the combustion front. 

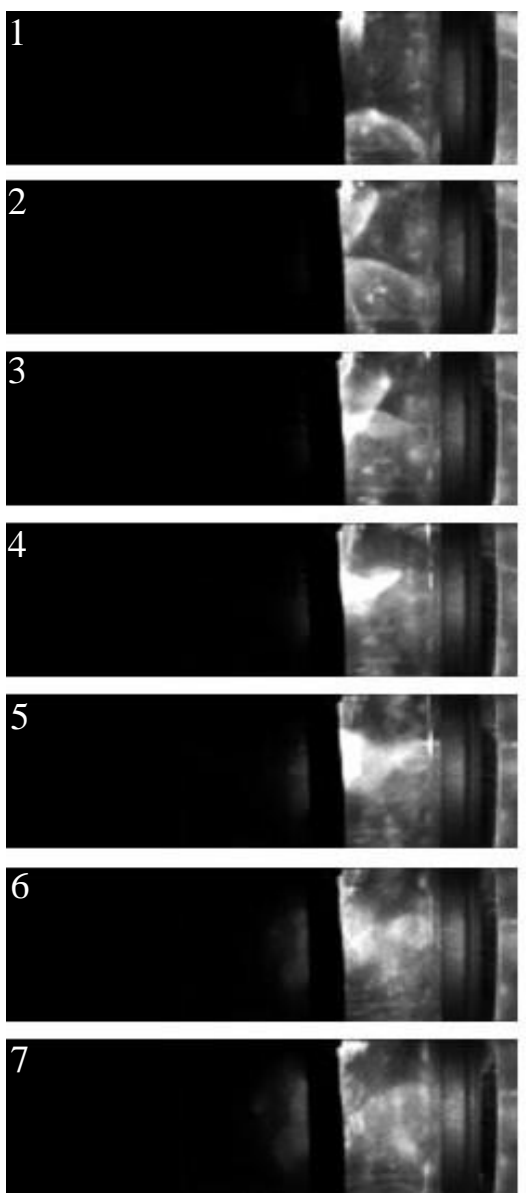
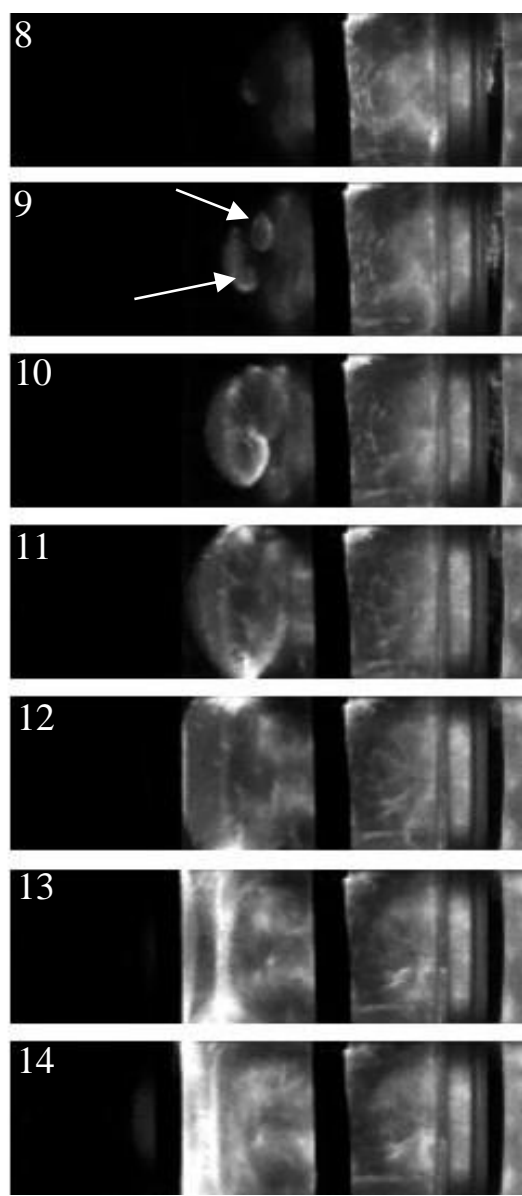

Figure 4.33: Side-view video from a $55 \mathrm{kPa}$ test with $75 \% \mathrm{BR}$, filmed at 300,000 fps (Test 243)

As seen in Figure 4.33, the detonations 'catch-up' to the combustion front and influence the detonation propagation speed. However, in the pressure range being considered detonations also appeared to be initiated at multiple points on the tube wall behind the combustion front and therefore may not have influenced the overall propagation speed. Detonation initiation behind the detonation front is captured in the side-view video recorded at 70,000 fps for a $60 \mathrm{kPa}$ test, shown in Figure 4.34. After the detonation wave passes through OP 6 (shown in Image 3), a single detonation kernel forms on the tube wall indicated by the arrow in Image 4. The video does not have a sufficient frame rate to show the kernel growing. In Image 6, multiple detonation kernels form on the tube wall. This video sequence also emphasizes that there 
is not a single detonation propagation mode - detonations may be initiated at a single point between a pair of orifice plates, such as in Image 4, and at multiple points just one obstacle pair later (as seen in Image 6).
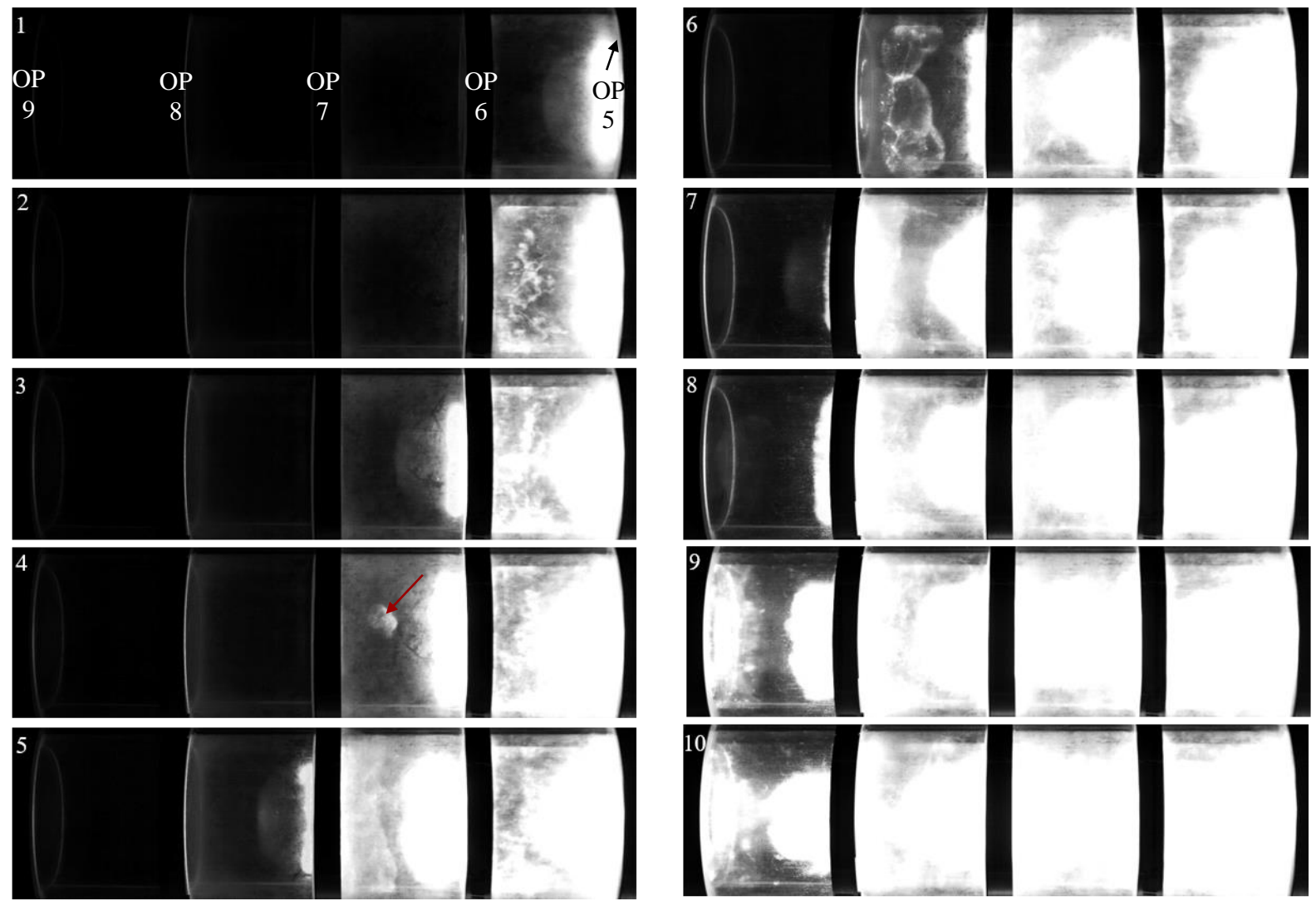

Figure 4.34: Side-view video for a $60 \mathrm{kPa}$ test with $75 \% \mathrm{BR}$ captured at 70,000 fps (Test 99)

To better understand the detonation initiation mechanism, a close-up of detonation initiation occurring on the wall for a $60 \mathrm{kPa}$ test was filmed at 175,000 fps and is presented in Figure 4.35. The fifth image of Figure 4.35 is similar to the sixth image of Figure 4.34, showing multiple detonation kernels forming on the wall. Image 1 shows the detonation wave emerging from the seventh orifice plate into the field-of-view and propagating towards the eighth orifice plates in Images 2 and 3. The detonation wave is travelling at velocity of approximately $2400-2700 \mathrm{~m} / \mathrm{s}$ along the centerline, indicating that the detonation has not failed. Multiple detonation kernels form on the tube wall near the eighth orifice plate, as shown in Image 5. The detonation kernels are no longer visible in the sixth image as they have run into the region of 
burned gas. It is believed that the ring of detonation kernels do not contribute to the centreline velocity and are similar to the detonation bubbles observed in the continuous detonation mode for the $50 \% \mathrm{BR}$.
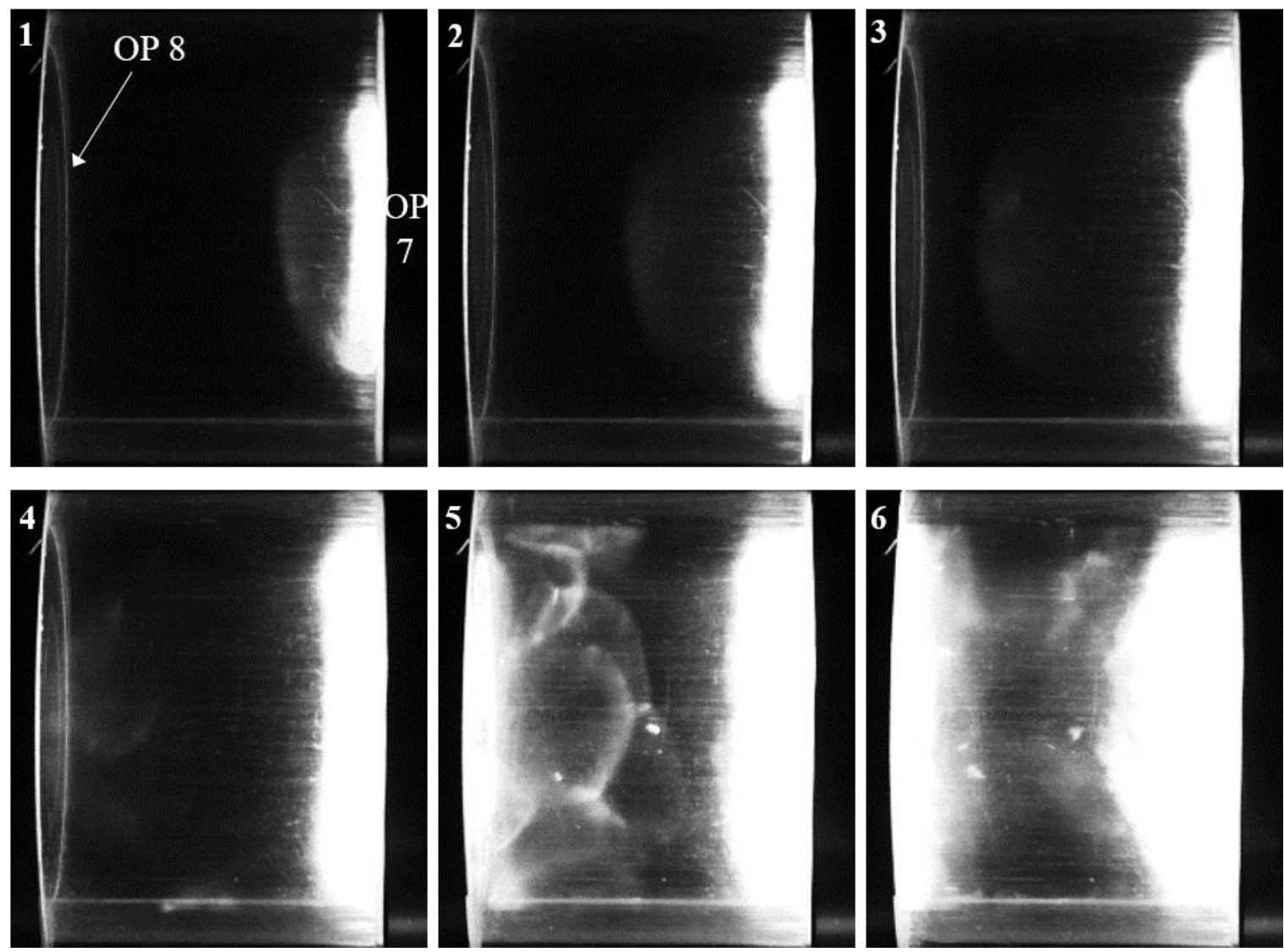

Figure 4.35: Close-up of a $60 \mathrm{kPa}$ test with $75 \%$ BR showing multiple detonation initiation points on the all, filmed at 175,000 fps between Orifice Plates 7 and 8 (Test 105)

A curved soot foil (shown in Figure 4.36) was installed for the $60 \mathrm{kPa}$ test shown in the video above (Figure 4.35). The field-of-view for the video shown in Figure 4.35 was between OP 7 and OP 8 and the soot foil was positioned between OP 9 and 10. There are at least six detonation kernels that form immediately before OP 10, indicated by arrows. There are white ' $y$ ' shaped and vertical lines (circled in red) etched in the soot, which are generated from strong shocks colliding with the tube wall. This soot foil is similar to the soot foil obtained for the $60 \mathrm{kPa}$ test (continuous detonation mode) with the 50\% BR. It 
does not have the characteristic peaks seen on the foils obtained for the multi hot-spot wall ignition mode at the $50 \%$ BR or in [6].

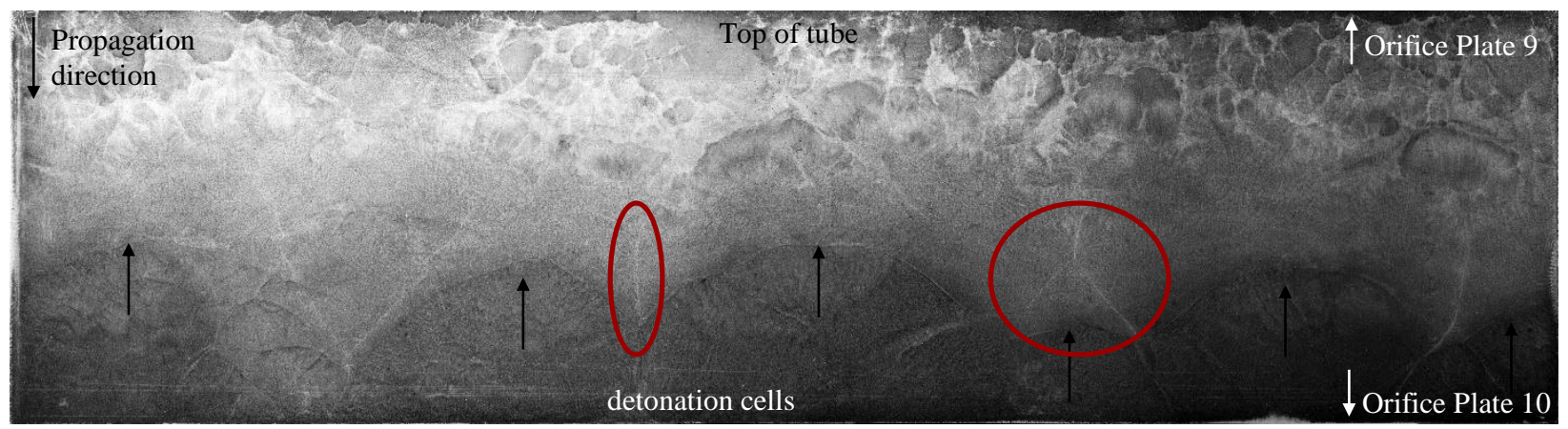

Figure 4.36: Curved soot foil for $60 \mathrm{kPa}$ test with $75 \%$ BR showing multiple detonation initiation points (Test 105)

Although there were instances where the detonation did not appear to fail between an individual pair of orifice plates, this detonation mode was not classified as a continuous detonation mode because of the variability in detonation initiation modes (i.e. a detonation did not propagate continuously down the length of the cylindrical channel). A flat soot foil was installed at the channel centreline for a $60 \mathrm{kPa}$ test to confirm that a detonation was not continuously propagating between orifice plates. There are detonation cells on the right side of the flat foil (shown in Figure 4.37), as the detonation which is propagating right to left emerges from the fourteenth orifice plate. However, there are no detonation cells on the left side of the foil (shown in the circle), indicating that the detonation completely failed along the channel centreline between orifice plates. Like the centreline foils obtained for continuous detonation propagation in the 50\% BR tests (example provided in Figure 4.13), the cell structure is lost at the top and bottom of the foil where the detonation fails due to diffraction. All that remains are parallel triple-point lines that approach the tube wall. It is believed that the collision of these triple-points with the wall are responsible for the detonation kernels observed in the video and the fine cells inside of the domes in Figure 4.36. 


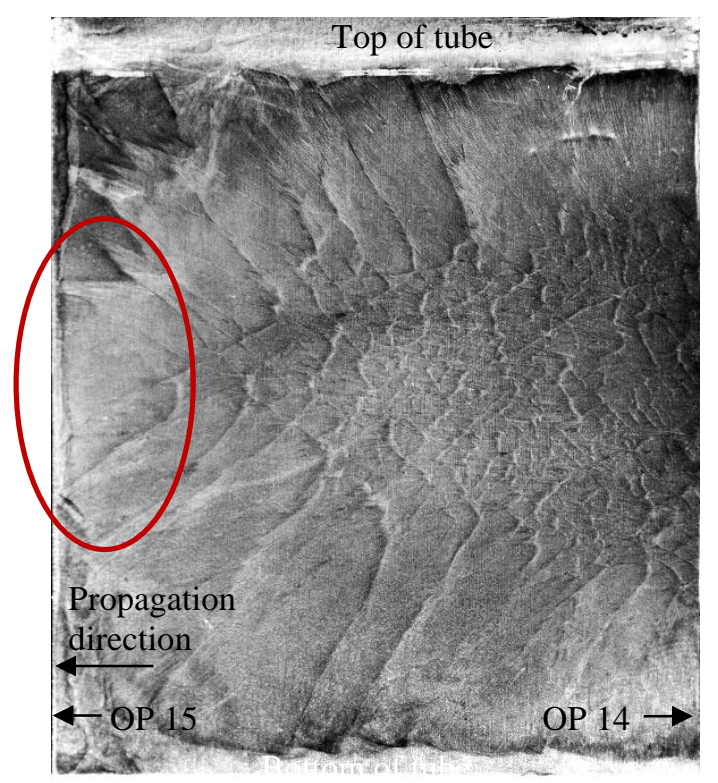

\section{Figure 4.37: A centreline soot foil installed vertically between OP 14 and 15 for a $60 \mathrm{kPa}$ test with $75 \%$ BR (Test 278)}

Video corresponding to the foil presented in Figure 4.37 is shown in Figure 4.38. The video was recorded between the fourteenth and fifteenth orifice plates (the same pair of orifice plates which the foil was installed between). Because the soot foil was installed vertically between the plates, only the front half of the channel is visible in the video. The video is brighter than similar videos recorded without a soot foil as the soot incandesces generating additional light.

In the first image of Figure 4.38, the detonation wave is shown emerging from the fourteenth orifice plate. Most of the combustion front is very bright. The brightness provides a relative measure of temperature, where the highest temperature region occurs where a detonation is propagating. The bright, high-temperature region of the combustion front is a detonation, as confirmed by the presence of detonation cells at the corresponding location on the foil shown in Figure 4.37. As the detonation wave propagates towards the fifteenth orifice plate, the detonation begins failing at the tube wall, as seen by the corrugated front (indicated by arrows in Images 2 and 3). In Images 2 and 3, the combustion front is brighter at the channel centerline (where a detonation is propagating) than at the tube walls (where the detonation has failed). The triangular pattern of detonation cells on the foil indicates that the detonation fails at the tube 
wall first. By the fourth image, the detonation has completely failed along the channel centerline, as confirmed by the absence of detonation cells in the foil. At this point, the combustion front has an approximately uniform level of brightness. Note that the detonation wave is highly curved due to the effect of diffraction past the orifice plate throughout the video sequence.
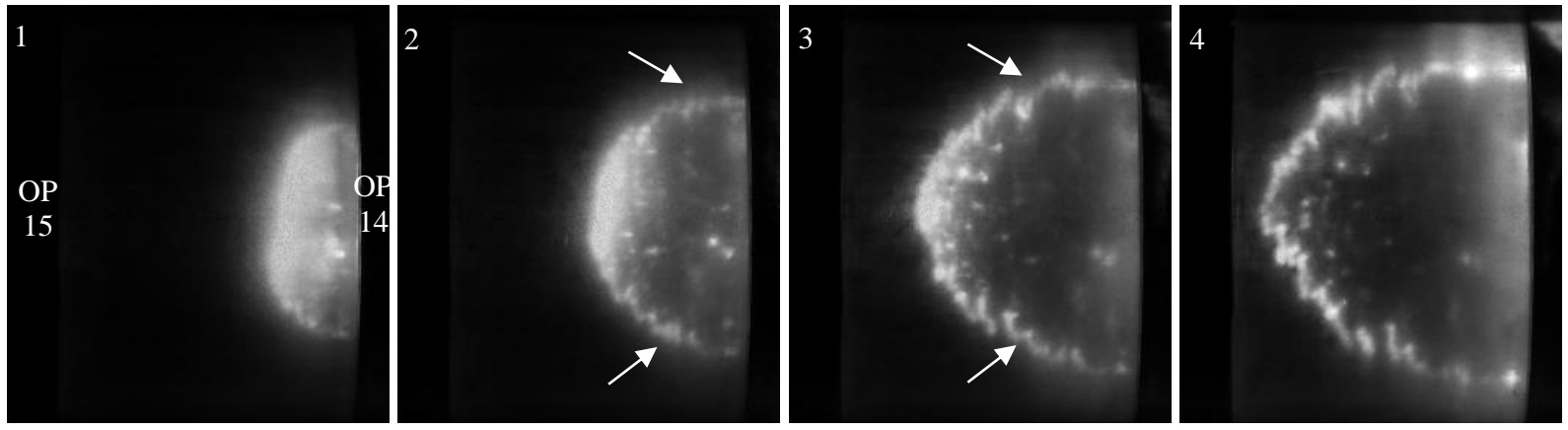

Figure 4.38: Video footage of a $60 \mathrm{kPa}$ test with $75 \% \mathrm{BR}$ filmed at 175,000 fps (Test 278)

It is expected that a continuous detonation propagation mode would occur at higher initial pressures but these were not tested due to the pressure constraints of the tube.

\subsubsection{Ignition behind the Flame Front}

An interesting phenomenon was observed for tests below the DDT limit (in the range of $20-35$ $\mathrm{kPa}$ ), where ignition occurred at a hot-spot far behind the combustion front. It is important to note that this behaviour is not captured in the average combustion front velocity data (shown in Figure 4.23), and would not be observed using non-optical techniques because it is occurring behind the main combustion front (and lead shock). This phenomenon is also observed for some tests in the first half of the tube while the combustion wave is still transitioning after the square-to-cylindrical geometry change. A video showing this phenomenon for a $30 \mathrm{kPa}$ test is presented in Figure 4.39. The detonation is initiated at a single point at $\mathrm{OP} 10$, shown in Image 14. In Images 15 - 20 the detonation wraps around the inner circumference of the tube (similar to that observed in Figure 4.26), spreading through the region of shock compressed 
unburned gas around the wall. The detonation occurs well behind the flame front which has almost reached OP 10 at this point.
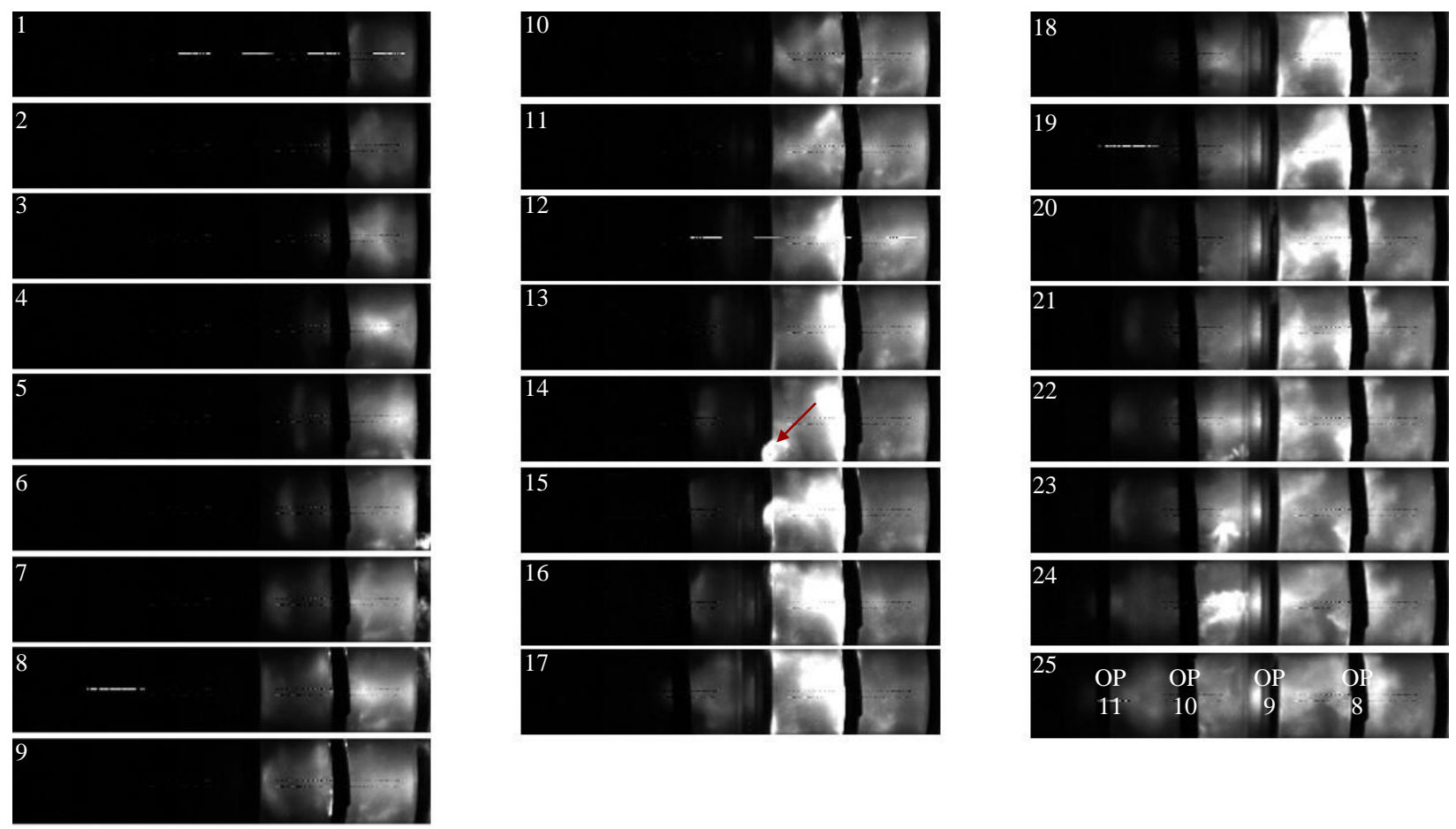

Figure 4.39: Side-view of a $30 \mathrm{kPa}$ test with $75 \%$ BR showing detonation occurring at the obstacle face behind the flame front, filmed at $300,000 \mathrm{fps}$, but presented with an effective frame rate of 100,000 fps Note that the horizontal dotted-lines seen in the images are a result of issues with the camera. (Test 274)

A similar video, shown in Figure 4.40, for a $35 \mathrm{kPa}$ test has been cropped to just show the wave progression between two orifice plates (OP 18 and 19). The video sequence was filmed at 300,000 fps. Detonation initiation occurs in Image 3, as indicated by the white arrow. Initiation likely occurs on the side of the orifice plate face closest to the back and top of the tube. The wave can be seen spreading around the inner circumference of the tube in Images $4-15$, before the two sides of the detonation collide in Image 16. To determine whether this wave was a detonation, the same approach used in Section 4.2.3.2 was used. The resulting wave speed of $3000 \mathrm{~m} / \mathrm{s}$ confirmed that this was a detonation. 

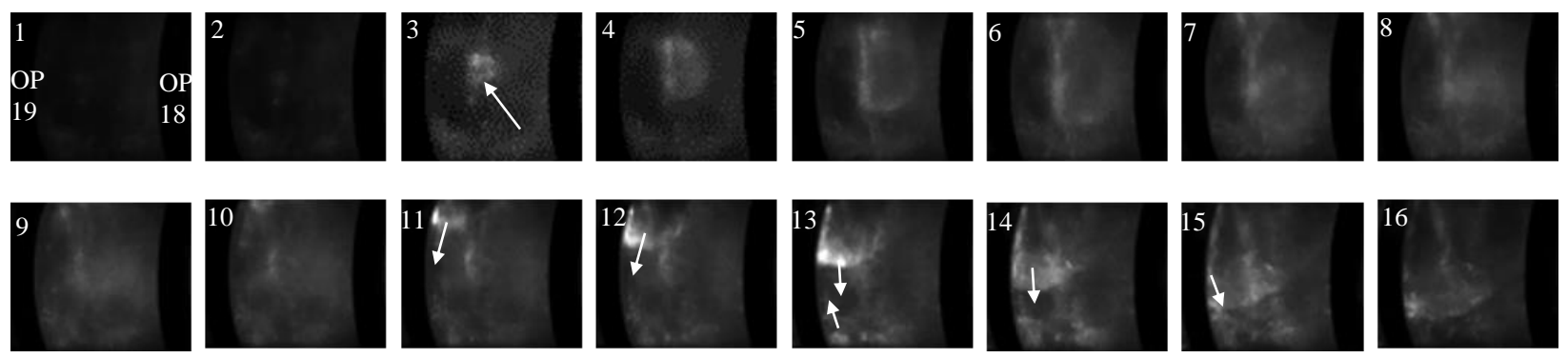

Figure 4.40: Video filmed at 300,000 fps showing a 'wrap-around detonation' occurring behind the combustion front for a $35 \mathrm{kPa}$ test with $75 \% \mathrm{BR}$ (Test 241)

Figure 4.40 shows video frames from a test where the detonation propagated around the entire circumference of the tube; however, at lower pressures there are cases where hot-spots ignite and fail before traversing all the way around the tube. An example of this is shown in Appendix A.

In order to understand the mechanism behind these late hot-spot ignitions, the flame structure behind the obstacle must be considered. A highly convoluted flame approaches the obstacle, as shown in a series of Schlieren images from the square channel for a $25 \mathrm{kPa}$ stoichiometric hydrogen-oxygen test [10] (Figure 4.41). Boeck et al. [10] conducted their experiments using the same square combustion channel equipped with fence-type obstacles (shown by the black rectangles) used in this research. The Schlieren images show the shock waves and turbulence, but the flame is not visible. There is a highly turbulent environment behind the obstacle which can facilitate ignition, as seen in Figure 4.40. The red arrow in Image 5 of Figure 4.41 shows where hot-spot ignition behind the obstacle face occurs. 


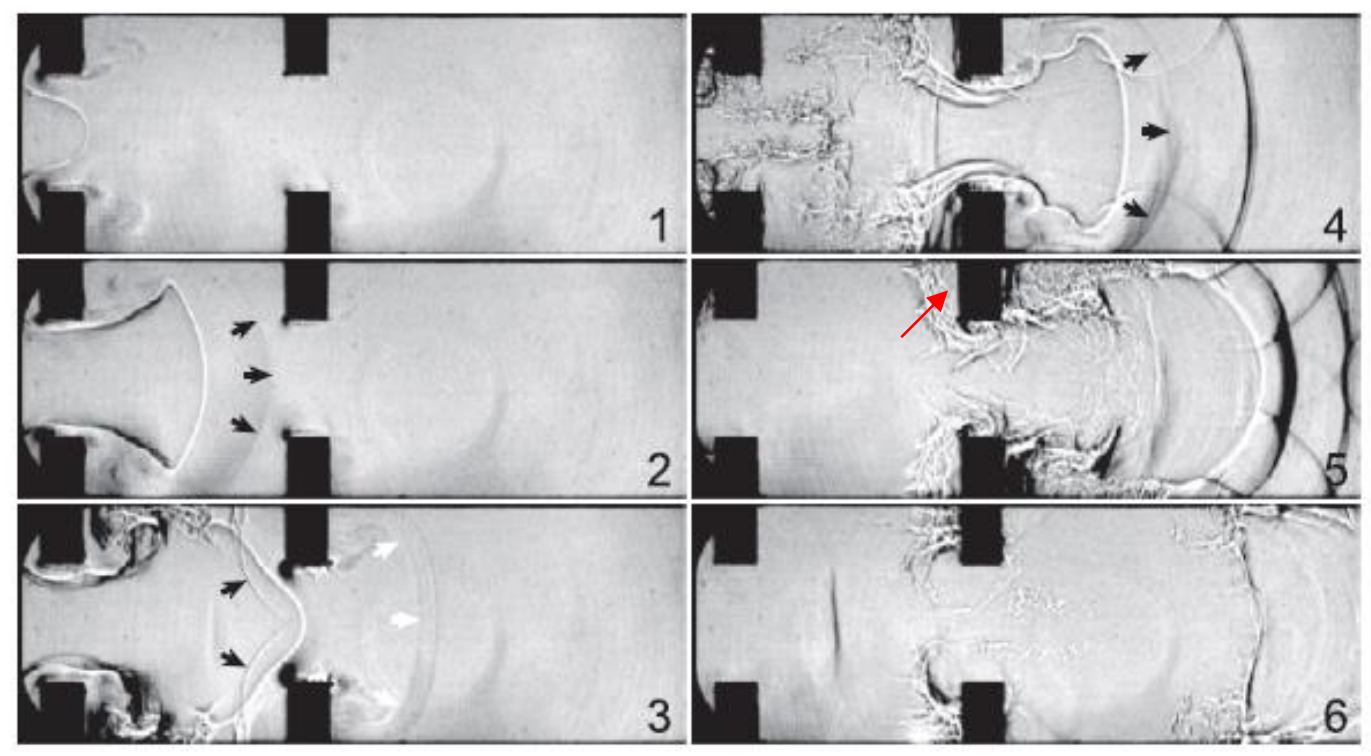

Figure 4.41: Schlieren images of a $25 \mathrm{kPa}$ stoichiometric hydrogen-oxygen test completed in the square channel showing the turbulent flame structure approaching the obstacle [10]

Ignition behind the flame front was not observed in the 50\% BR tests. A possible explanation for this is that based on the height of the orifice plates. The higher BR plates allow for a larger region of turbulence in which ignition can occur.

This propagation mechanism can be represented schematically as shown in Figure 4.42. As seen in the schematic, the flame front and lead shock are far ahead of where the detonation is initiated. The detonation path through the unburned gas along the circumference of the tube is indicate by the grey dashed arrows. 


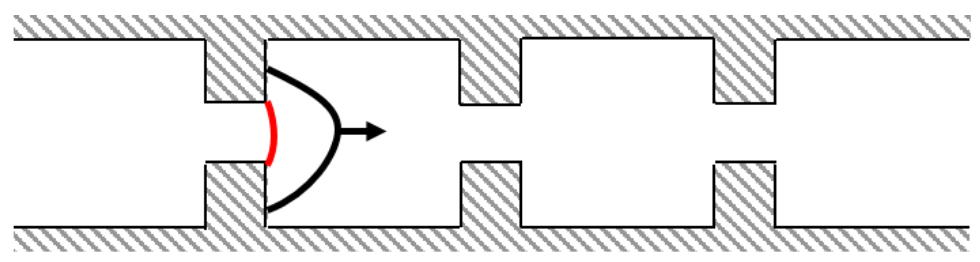

Choked flame propagation

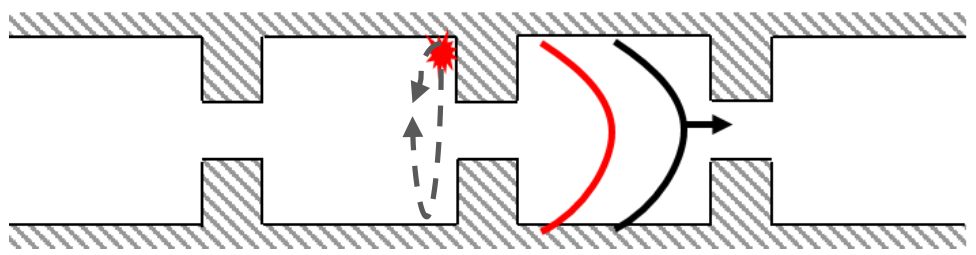

Detonation initiation behind flame front

Figure 4.42: Schematic showing detonation initiation due to turbulent mixing far behind the flame front

\subsubsection{Variation in Quasi-Detonation Propagation Modes}

In the $75 \%$ BR tests, a significant variation in local propagation velocity between orifice plates was observed, even at high initial pressures. This variation was captured in the velocity profiles for different 50 - $60 \mathrm{kPa}$ tests, shown in Figure 4.43. As in Figure 4.22, the average combustion front velocities were determined according to the procedure outlined in Section 3.4.2 for the 'steady-state tests'. Figure 4.43 shows the velocity fluctuations as the combustion wave propagates down the channel. The highest uncertainty associated with the velocity measurement is $\pm 250 \mathrm{~m} / \mathrm{s}$ (the full uncertainty analysis is provided in Appendix B). As in Figure 4.22, the average propagation velocity between two orifice plates is plotted at the downstream orifice plate, and the square channel velocity entering the cylindrical channel is plotted at Obstacle 0.

The variation in local propagation velocity seen in the $75 \%$ BR tests was not observed in the $50 \%$ BR tests (as seen in Figure 4.1) or in [4] who noted only slight velocity fluctuations were seen between different sets of obstacle for their experiments completed with a $45 \%$ and $55 \%$ BR. 


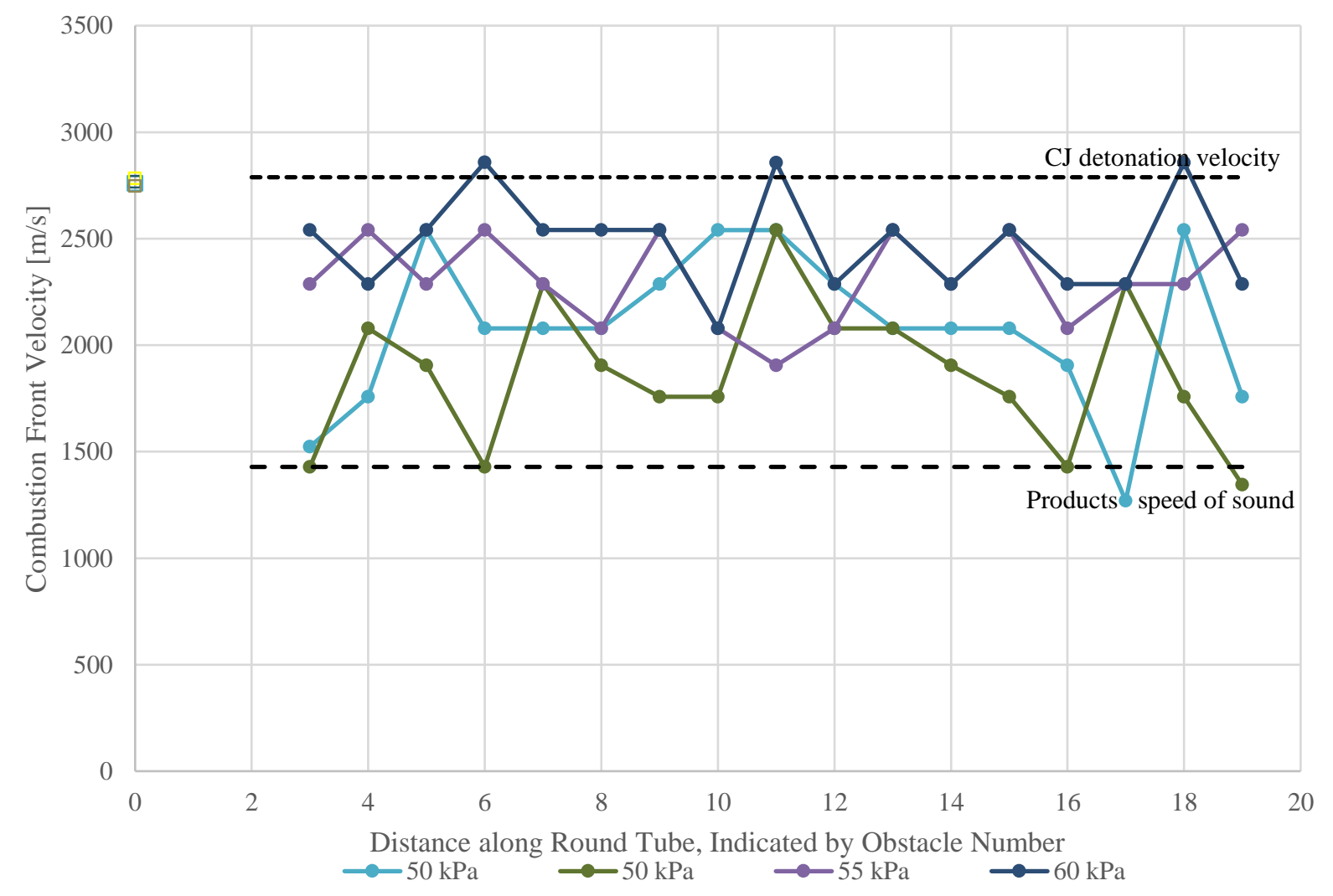

Figure 4.43: Plot of average combustion front velocity along the entire round tube for initial pressures between 50 and $60 \mathrm{kPa}$ showing the variation in propagation speeds

The variation in quasi-detonation propagation modes was also evident in the video footage recorded for the $75 \%$ BR tests. A portion of the video footage obtained for a $55 \mathrm{kPa}$ steady-state test filmed at 300,000 fps is shown in Figure 4.44. To ensure that the field-of-view was far enough downstream of the square-toround tube transition and therefore steady-state behavior was observed, this video sequence focuses on combustion wave propagation between Orifice Plates 14 and 18 (the same field-of-view considered for the 'full-size' field-of-view tests filmed at 70,000 fps). Three different detonation initiation modes are observed within the test, highlighting the variability in modes. Between OP 14 and 15, the multi-hot-spot wall ignition detonation mode is observed. Two hot-spots (indicated by the white arrows in Image 8) form on the tube wall and grow radially as seen in Image 9. A detonation is initiated from a single hot-spot on the tube wall between OP 15 and 16, indicated by the white arrow in Image 18. Between OP 16 and 17, detonation 
initiation occurs at multiple points on the obstacle face, as seen in Image 33. Farther downstream, detonations are initiated at two hot-spots on the wall between OP 17 and 18 in Image 38. 

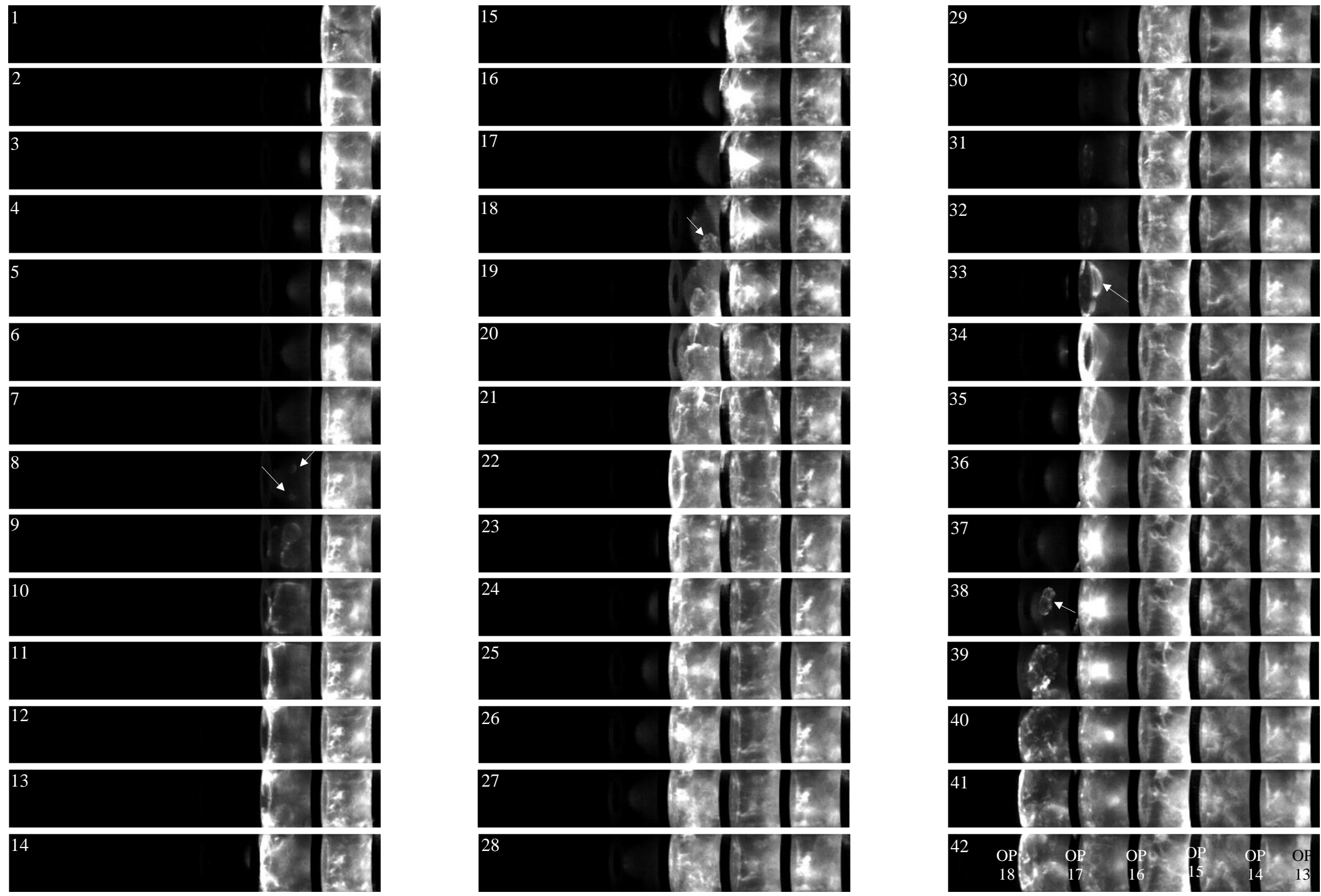

Figure 4.44: Side-view filmed at 300,000 fps of a $55 \mathrm{kPa}$ test with $75 \%$ BR showing hot-spot ignition on the wall (Test 243 ) 110 
To determine the relationship between detonation propagation mode and combustion front propagation velocity, the velocity profiles generated from the steady-state tests and their corresponding videos were analyzed. A substantially higher propagation velocity was observed when a detonation was initiated on the wall compared to at the obstacle face. Tests where obstacle face ignition was the dominant or sole detonation propagation mode exhibited an average combustion front velocity below the speed of sound in the products.

To illustrate this relationship, the combustion front velocity profile along the cylindrical tube for a $45 \mathrm{kPa}$ test is presented in Figure 4.45. The 'steady-state test' configuration was used and the propagation velocity was determined according to Section 3.4.2. The velocity point plotted at an obstacle number represents the average velocity between that $\mathrm{OP}$ and the previous OP (i.e. the data point at OP 10 represents the average velocity between OP 9 and 10). To avoid transition effects resulting from the change in squareto-cylindrical geometry, only detonation propagation in the second half of the tube (after OP 9, as indicated by the grey dashed line) was considered.

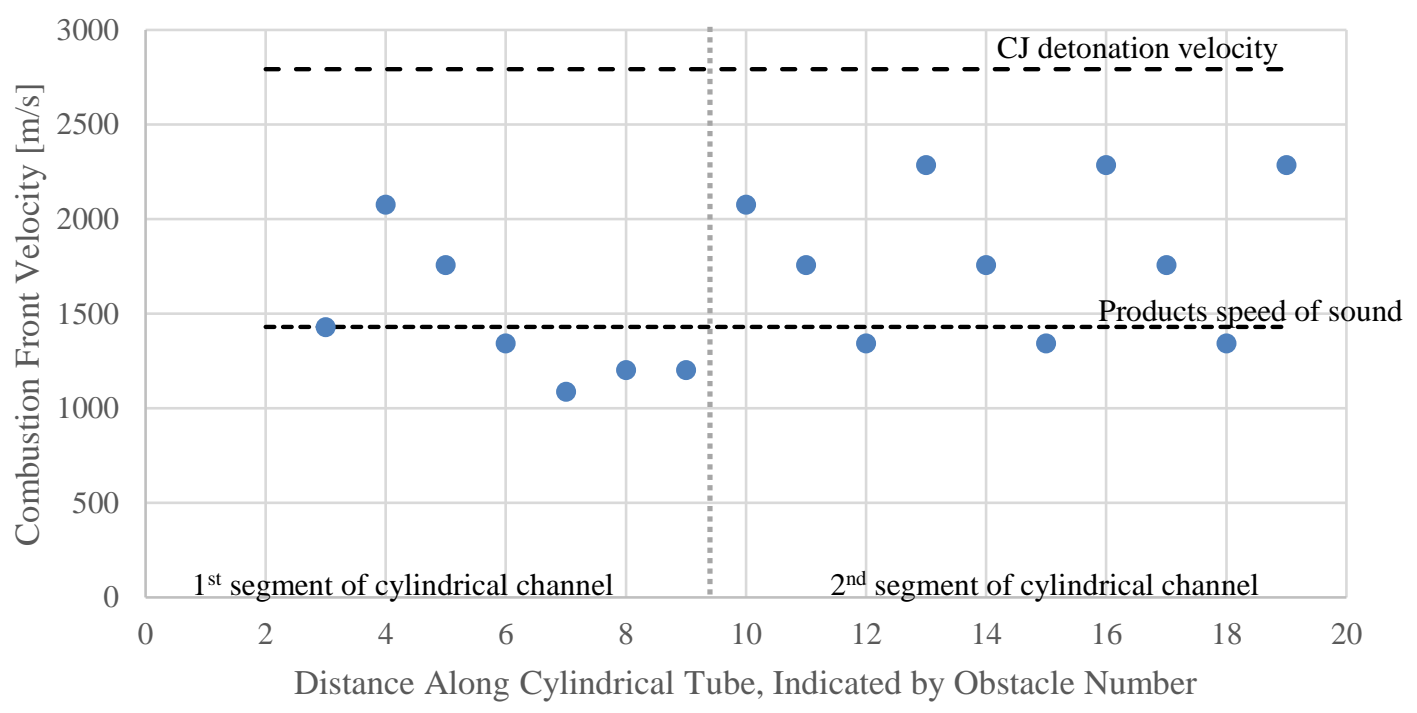

Figure 4.45: Combustion front velocity profile for a $45 \mathrm{kPa}$ test with $75 \%$ BR (Test 246) 
The corresponding video was carefully examined to correlate the propagation mode to the average velocity between each pair of orifice plates. A sequence of still images from the video, spanning OP $9-18$, is presented with an effective speed of $200,000 \mathrm{fps}$ in Figure 4.46. The velocity peaks (indicated by propagation velocities greater than $2000 \mathrm{~m} / \mathrm{s}$ ) seen in Figure 4.45 occur at OP 10, 13, 16 and 19 and represent a detonation which initiated from a hot-spot (or multiple hot-spots) on the tube wall. These detonation initiation points are indicated by white arrows in Images 4, 24 and 44 of Figure 4.46. Due to space constraints, the wall hot-spot detonation which initiated between OP 18 and 19 is not shown.

A different detonation propagation mode is observed between the remaining orifice plate pairs. At Orifice Plates 11, 12,14,15, 17 and 18, detonations are initiated at the obstacle face (as indicated by the red circles in Images 12, 21, 33, 41, 53 and 61). This different detonation mode is reflected in the velocity profile given in Figure 4.45 as propagation velocities less than $2000 \mathrm{~m} / \mathrm{s}$. A characteristic 'saw-tooth' pattern can be seen in the velocity profile as a detonation is initiated on the wall generating a high velocity. Subsequent detonations initiated on the obstacle face generate lower propagation velocities, leading to a decay in combustion front velocity. The momentum generated during the wall ignition detonation propels the wave forward so the average propagation velocity is higher between the next two orifice plates immediately following wall ignition, but it continues to drop. As shown in Section 4.2.3.2 the obstacle face ignition detonation does not increase the combustion front velocity over that of a fast-flame. The next sawtooth peak is formed by a hot-spot wall ignition detonation. 

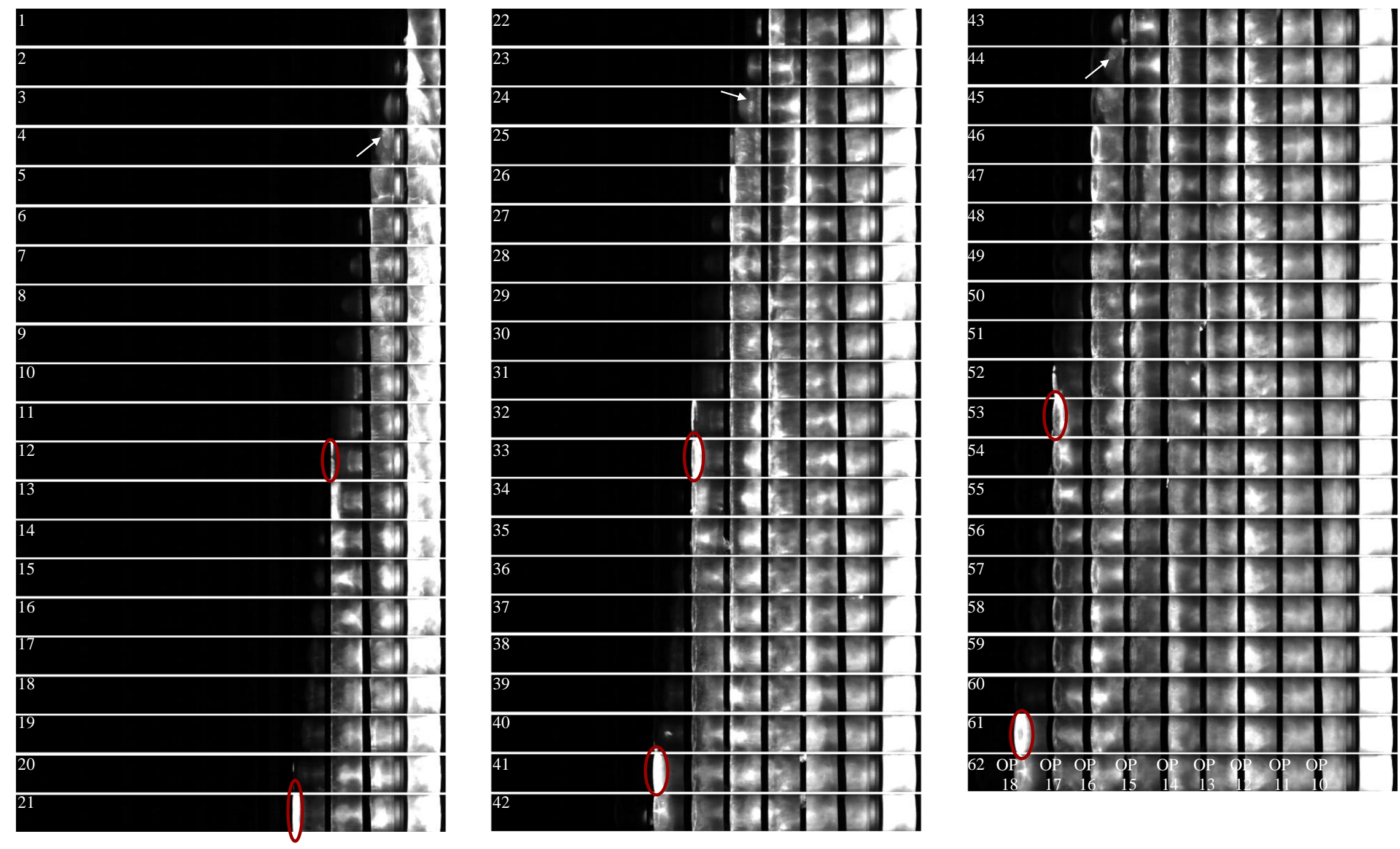

Figure 4.46: Side-view video footage of a $45 \mathrm{kPa}$ test, originally filmed at $300,000 \mathrm{fps}$ presented with an effective frame rate of $200,000 \mathrm{fps}$ (Test 246) 


\subsection{Detonation Propagation Limits}

A comparison of the average combustion front velocities obtained in the cylindrical channel for the $50 \%$ and $75 \%$ BR tests as a function of initial pressure is presented in Figure 4.47. Peraldi [5] established that in order for DDT to occur, the orifice diameter must be equal to, or greater than, one detonation cell size (i.e. the critical $d / \lambda \geq 1$ ). In the present research, experiments performed with a $50 \%$ BR indicated a $d / \lambda$ of 1.4 at the detonation limits, which is consistent with Peraldi's criterion. The detonation limit in the square channel corresponded to a $\mathrm{d} / \lambda=1.6$, which is also consistent with Peraldi's criterion. As expected, the detonation limit for the $75 \%$ BR tests was significantly higher than the detonation limit for the $50 \%$ BR tests. The detonation limit for the $75 \%$ BR tests (based on the average velocity data and defined as an average velocity greater than the products speed of sound) corresponded to a $d / \lambda=14$. Detonations were observed in lower pressure tests $(35 \mathrm{kPa})$, although the average combustion front velocities for these tests were less than the products speed of sound and therefore $35 \mathrm{kPa}$ is not classically defined as the detonation limit. Based on the cell size data, tests completed at $35 \mathrm{kPa}$ corresponded to a $\mathrm{d} / \lambda=10$. Although this does not violate Peraldi's $d / \lambda>1$ criterion which permits DDT, it is significantly higher than the critical condition of $d / \lambda=1$ for the onset of DDT. 


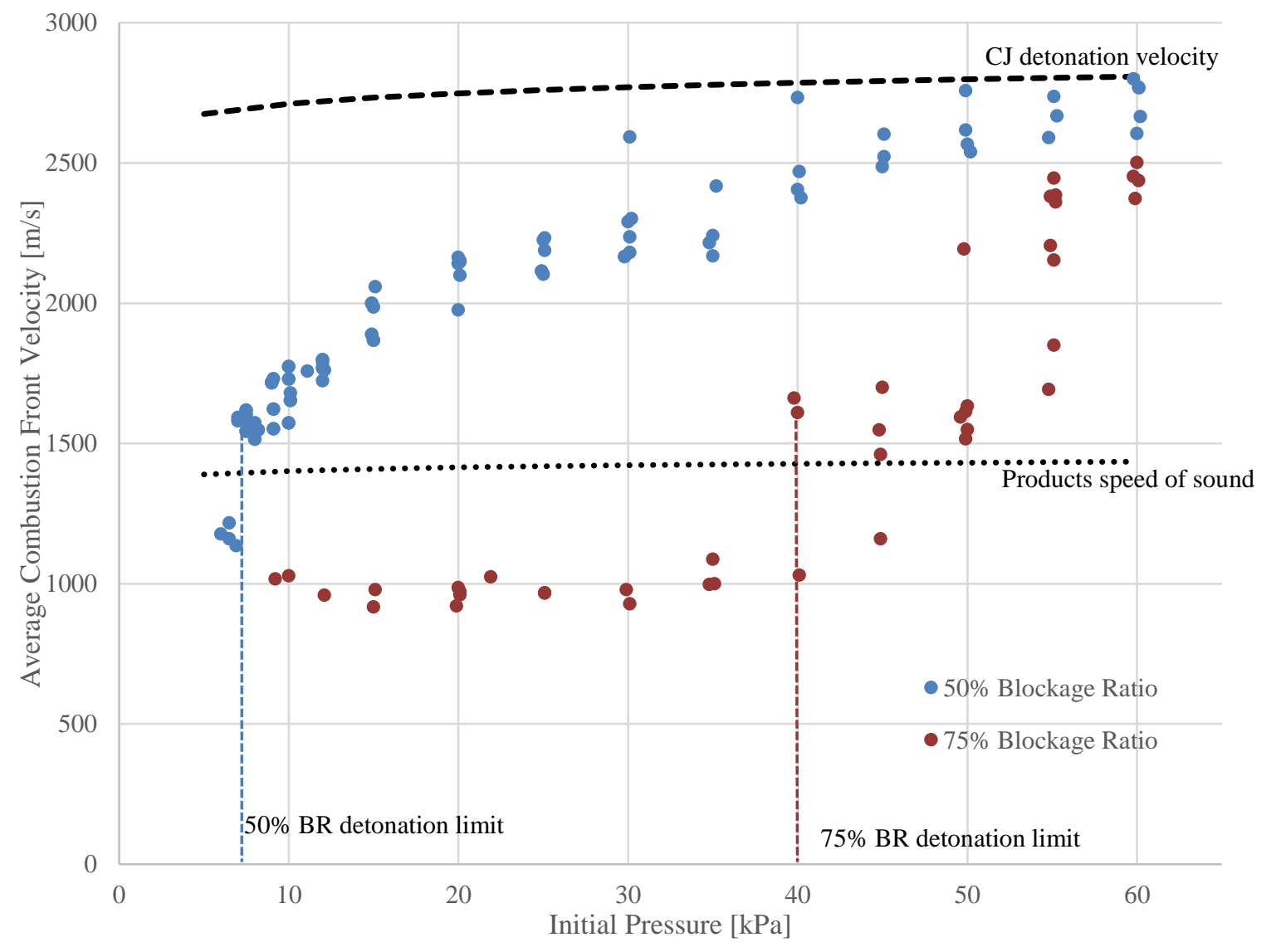

Figure 4.47: Comparison plot of average combustion front velocities in the cylindrical channel for $B R=0.5$ and $B R=0.75$ as a function of initial pressure

The difference in critical $\mathrm{d} / \boldsymbol{\lambda}$ observed in the present research is consistent with experiments reported in [11] and [43], who showed that the critical $d / \lambda$ is influenced by blockage ratio. They demonstrated that as blockage ratio increases, the critical $\mathrm{d} / \lambda$ increases. However, the cause for this increase was not adequately explained.

The visualization used in this study allowed for a comparison of detonation initiation modes at the limit. The difference in critical $\mathrm{d} / \lambda$ between blockage ratios may be attributed to the difference in near-limit detonation initiation modes. In the $50 \%$ BR tests, at the propagation limit $(7-15 \mathrm{kPa})$ detonations were consistently initiated at a single site on the tube wall following shock reflection. However, a different detonation initiation mode was observed at the propagation limit in the $75 \%$ BR tests. The first sustainable 
mode observed (for initial pressures of approximately 35 - $40 \mathrm{kPa}$ ), relied on shock reflection at the orifice plate face to initiate a detonation. The smaller orifice diameter (for the $75 \% \mathrm{BR}$ ) resulted in a farther distance for the decoupling detonation wave to propagate during diffraction before reaching the tube wall. Because of this longer distance, for the same shock strength entering the orifice plate the lead shock wave weakens more and is less likely to result in detonation initiation at the tube wall. Based on the visualization, the shock reflection forms a Mach stem that subsequently reflects off the orifice plate surface producing a hot-spot that forms into a detonation. At higher initial pressures, a detonation can initiate at a hot-spot on the wall since the shock strength before diffraction is stronger.

\subsection{Critical Tube Diameter}

The well-established critical tube diameter criterion $d_{c} / \lambda \approx 13$ governs the conditions under which a detonation wave can survive a severe diffraction like the transmission of a wave from a confined tube into open space. Using an orifice plate position at the tube exit, Liu and Lee [36] showed that the critical tube diameter and critical orifice plate diameter are essentially the same. Pantow et al. [40] demonstrated that the addition of boundaries to the receiver volume which facilitate shock reflection, reduces the minimum $d / \lambda$ value required to achieve re-initiation of the detonation, effectively reducing the required critical tube diameter. In a round tube equipped with orifice plates, like the present experiments, the transmission of the detonation through the orifice plate can be analyzed as a critical tube phenomenon. Because of the presence of tube walls in the experimental apparatus used, it would be expected that continuous detonation propagation would be observed at $\mathrm{d} / \lambda \approx 13$ or less.

For the $50 \%$ BR configuration, the continuous detonation regime, in which a detonation propagates without failing along the channel centreline, starts at an initial pressure of $50 \mathrm{kPa}$ (corresponding to a d/ $\lambda$ of 25). Based on the cell size data presented in Appendix $C, d / \lambda=13$ (the ratio established in literature)

corresponds to an initial pressure of approximately $35 \mathrm{kPa}$. At this initial pressure in the present experiments, detonations are initiated at multiple sites on the tube wall. 
A centerline soot foil obtained at $30 \mathrm{kPa}(\mathrm{d} / \lambda=9$, based on Appendix $\mathrm{C})$ is presented in Figure 4.48. As the detonation passes through the orifice plate, expansion waves starting at the orifice edge move inwards towards the centerline reducing the pressure behind the lead shock wave. This expansion is responsible for the cooling of the reaction zone and the decoupling of the shock and reaction zone. As the expansion waves propagate inward at the speed of sound of the products (roughly half the CJ velocity) there is a core region that is unaffected by the expansion. This core gets smaller with propagation distance of the lead shock wave, forming a cone whose base is the same dimension of its height. A cone of influence is drawn on the foil in Figure 4.47, where cells exist inside the cone and the cellular structure fails due to the decoupling of the detonation wave on either side of the cone. In this subcritical case (based on the critical tube criterion), the detonation wave does not reinitiate by shock reflection off the wall, but cells are present past the apex of the cone indicating the detonation wave does not fail. There are two anomalies in this foil, there is a lack of cells at the inlet core (see dotted circle) and the presence of a triple-point trajectory line parallel to the cone boundary, just to the left and right of the cone. These anomalies suggest that the detonation may not be planar exiting OP 8 . 


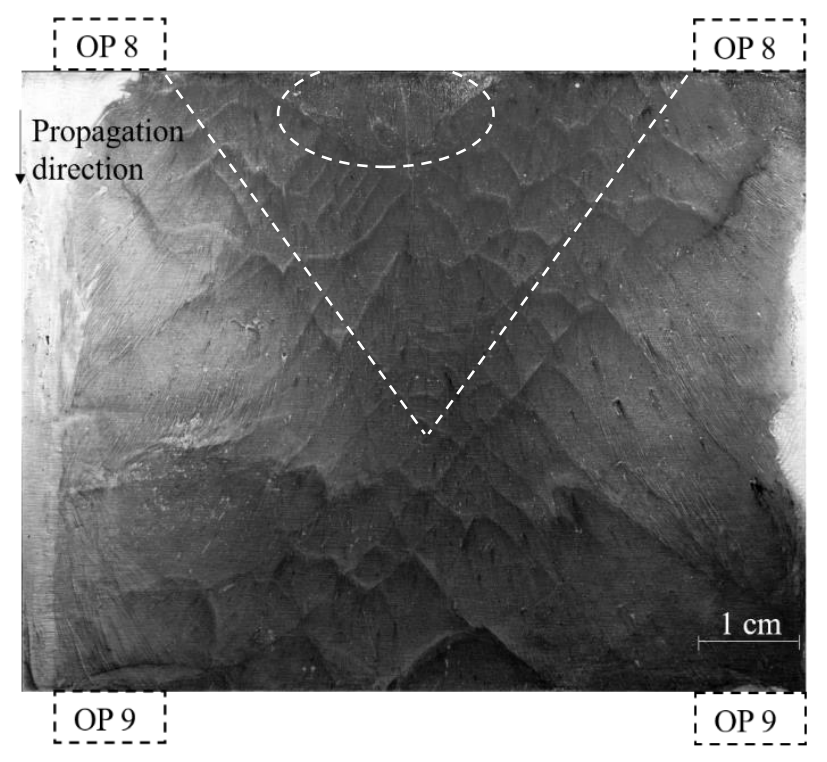

Figure 4.48: Centreline soot foil obtained from a $30 \mathrm{kPa}$ test with $50 \%$ BR (Test 161). The labeled dashed boxes indicate the relative position of the orifice plates.

There are several interesting features on the $40 \mathrm{kPa}$ foil shown in Figure 4.49. The same cone of influence as shown in Figure 4.48 is drawn, as the $10 \mathrm{kPa}$ increase in initial pressure does not significantly change the product speed of sound and CJ velocity. The initial test pressure (40 kPa) corresponds to a value of $d / \lambda=20$, which is supercritical based on the critical tube criterion and thus the detonation would be expected to survive the diffraction. Video footage (not shown) indicate multiple face ignition sites on the tube wall at this pressure. The cell structure on the foil is very asymmetric - there is a band of very fine cells, indicated by white dashed circle, on the left side of the cone region. This would indicate that an overdriven detonation wave enters on that side, most likely due to orifice plate face ignition. The cell structure fails on the right side of the cone but survives on the left side, stemming from the fine cell region. This case shows that although the resulting survival of the detonation wave is consistent with the critical tube criterion, the foil shows that the actual phenomenon is far from an ideal scenario with a planar, uniform detonation wave exiting the orifice. 


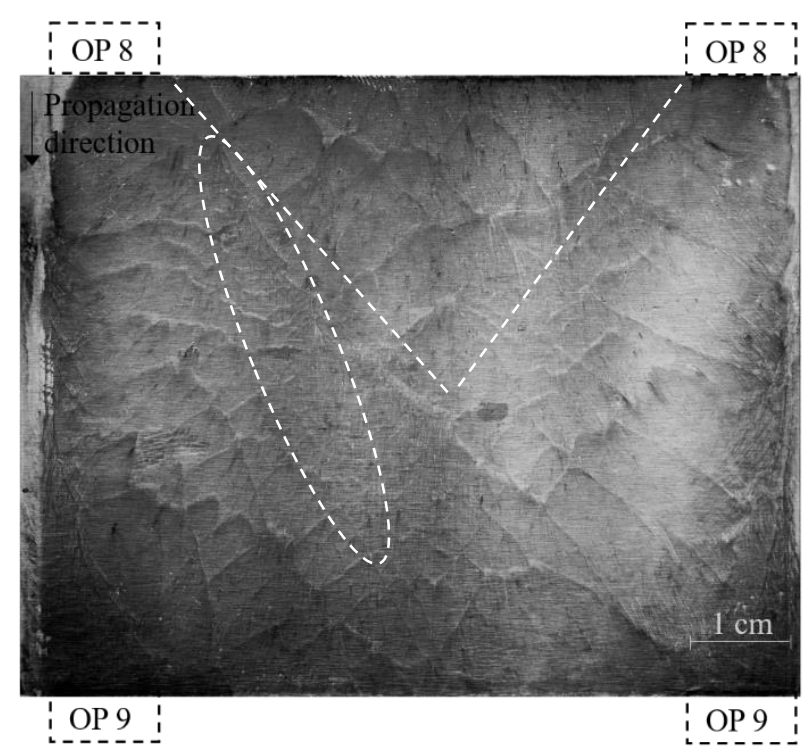

Figure 4.49: Centreline soot foil obtained from a $40 \mathrm{kPa}$ test with $50 \%$ BR (Test 158). The labeled dashed boxes indicate the relative position of the orifice plates.

A centerline foil for a $60 \mathrm{kPa}$ test, where continuous detonation propagation is observed, is shown in Figure 4.50. This pressure corresponds to a value of $d / \lambda=37$, based on the cell size data from Appendix C. Unlike the $30 \mathrm{kPa}$ and $40 \mathrm{kPa}$ foils, there are uniform cells along the channel centerline. From this foil, the average detonation cell size at the orifice plate was measured to be $1.7 \mathrm{~mm}$, giving a $\mathrm{d} / \lambda$ ratio of 31 . This is supercritical, based on the critical tube criterion, and thus the detonation would be expected to survive the diffraction. The cells increase in size towards the left and right edges of the foil where the detonation decouples at the tube wall. The cells continue in the core past the cone of influence, indicating the detonation wave survives the expansion. Although the detonation wave along the centerline survives, diffraction around the orifice plate introduces a curvature to the detonation wave (which cannot be seen on the foil), that can be seen in the video in Figure 4.14. This curvature results in the significantly lower propagation velocity relative to the $\mathrm{CJ}$ detonation velocity, which is based on the propagation of a planar wave. 


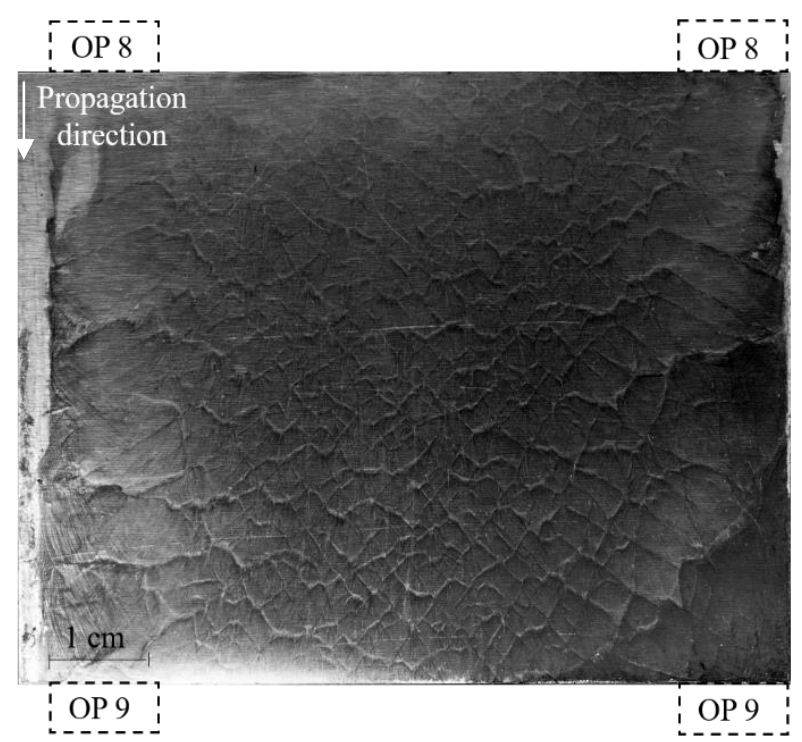

\section{Figure 4.50: Centreline foil for a $60 \mathrm{kPa}$ test with $50 \% \mathrm{BR}$ (Test 157). The labeled dashed boxes indicate the relative position of the orifice plates.}

The foils presented in Figure 4.48 and Figure 4.49, suggest that the critical tube criterion cannot be directly applied to this experiment because of the asymmetric nature of the detonation wave approaching the orifice, even though in some cases the detonation propagation results are consistent with the critical tube criterion. In this experiment, the critical tube condition only applies at very high pressures (e.g., 50 and $60 \mathrm{kPa}$ ) where a continuous detonation wave was observed.

As in the $50 \%$ BR tests, a $\mathrm{d} / \lambda$ value of 13 did not correlate to the continuous detonation mode for the $75 \%$ BR tests. According to Appendix $C, d / \lambda=13$ corresponds to an initial pressure of approximately $40 \mathrm{kPa}$, which was the detonation propagation limit for this orifice plate, as seen in Figure 4.23. Even the videos recorded for a $60 \mathrm{kPa}$ test (corresponding to a $\mathrm{d} / \lambda$ of 27 , which is supercritical based on the critical tube criterion) for the $75 \%$ BR tests indicate that there is not a planar wave entering and exiting the orifice plate.

A centreline soot foil obtained from a $60 \mathrm{kPa}$ test is shown in Figure 4.51. The cone of influence, indicated by dashed white lines, has been added to the foil. Detonation cells are clearly visible within the cone of influence but there is no cell structure outside of the cone, indicating that the detonation does not 
survive the diffraction. There are detonation cells immediately after the fourteenth orifice plate in the cone, but no cells before the fifteenth, showing that the detonation has failed and transitioned to a deflagration before reaching the fifteenth orifice plate. Based on the video results at this pressure, detonations are typically initiated at multiple points on the tube wall. The cellular structure seen on this foil is consistent with sub-critical detonation. As was the case for the $50 \% \mathrm{BR}$, the critical tube criterion cannot be used to predict the start of the continuous detonation mode in these experiments.

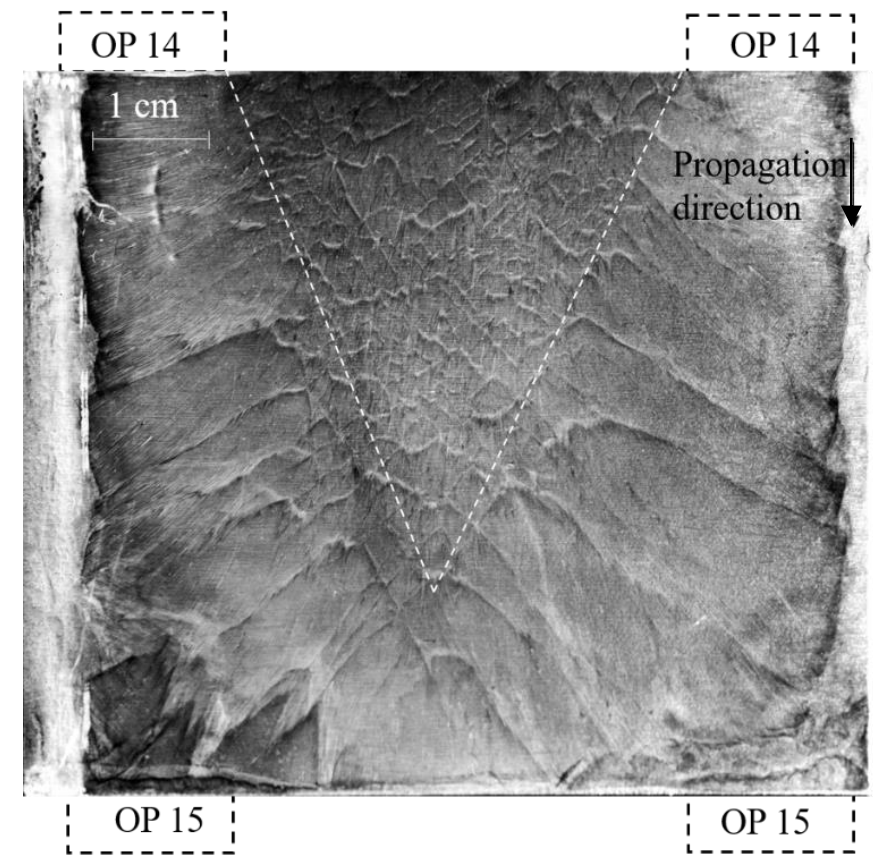

Figure 4.51: Centreline foil installed for a $60 \mathrm{kPa}$ test with $75 \%$ BR (Test 278). The dashed boxes indicate the relative positions of the orifice plates 


\section{Chapter 5}

\section{Conclusions and Recommendations}

The present research investigated quasi-detonation propagation and deflagration-to-detonation transition (DDT) using self-luminous high-speed photography in a transparent round tube equipped with repeating orifice plates. Tests were performed using stoichiometric hydrogen-oxygen mixtures at initial pressures of $4-60 \mathrm{kPa}$ in a square-to-round combustion channel. Supplemental detonation cell size data was collected in a $6.1 \mathrm{~m}$ long round detonation tube. Experiments were conducted using two sets of orifice plates with orifice diameters, d, of $5.33 \mathrm{~cm}$ and $3.81 \mathrm{~cm}$, representing $50 \%$ and $75 \%$ area blockage ratios (BR), respectively. Previous studies [11] [43] have demonstrated that the orifice plate diameter to detonation cell size ratio $(\mathrm{d} / \lambda)$ at the DDT limit increases with blockage ratio. However, these studies have failed to propose a mechanism for this relationship.

Experiments showed a clear difference in $\mathrm{d} / \lambda$ at the DDT limit between the two blockage ratios, based on the measured average combustion front velocity. The DDT limit (defined by the classical definition of an average combustion velocity greater than the products speed of sound) for the $50 \%$ BR tests was $7 \mathrm{kPa}$, which corresponded to a $\mathrm{d} / \lambda$ of 1.4 . A similar $\mathrm{d} / \lambda(\mathrm{d} / \lambda=1.6)$ was found at the limit in the square channel. The $\mathrm{d} / \lambda$ ratio at the DDT limit for both geometries for the $50 \% \mathrm{BR}$ is in agreement with Peraldi's critical $\mathrm{d} / \lambda \geq 1$ criterion [5]. The DDT limit for the $75 \%$ BR tests was significantly higher, occurring at 40 $\mathrm{kPa}$ and corresponding to a $\mathrm{d} / \lambda$ of 14 . Detonations were observed in $35 \mathrm{kPa}$ tests (corresponding to a d/ $\lambda$ of 10) although the average combustion front propagation velocity fell below the products speed of sound and therefore $35 \mathrm{kPa}$ is not classically defined as the DDT limit.

Visualization of the quasi-detonations revealed that this difference may be attributed to the difference in detonation initiation modes occurring near the limit. At the limit, for the $75 \%$ BR tests, 
detonations were primarily initiated off the obstacle face, instead of at a single hot-spot on the tube wall, as in the $50 \%$ BR tests.

High-speed camera footage, in conjunction with soot foils, was used to understand and classify the quasi-detonation propagation mechanisms. Three propagation modes were observed in the $50 \%$ BR tests. Near the limit $(7-15 \mathrm{kPa})$, detonations initiated at a single hot-spot on the tube wall. At higher initial pressures $(20-45 \mathrm{kPa})$, detonation initiation occurred at multiple hot-spots on the tube wall, and for very high pressure tests $(50-60 \mathrm{kPa})$, continuous detonation propagation was observed. At initial pressures less than $7 \mathrm{kPa}$, sustained fast-flame propagation was observed.

In the $75 \%$ BR tests, three quasi-detonation propagation modes were observed. There was a high degree of variability between tests, where multiple detonation modes were observed at the same initial pressure and even within the same test. This variation means that quasi-detonation propagation in the $75 \%$ BR tests cannot be classified into well-defined categories based on test pressure. Near the DDT limit (for initial pressures of $35-40 \mathrm{kPa}$ ) detonation initiation primarily occurred at the orifice plate. At higher initial pressures, detonations were also initiated at a single hot-spot on the tube wall and at even higher initial pressures, detonation propagation was primarily governed by detonation initiation at multiple hot-spots on the tube wall. The continuous detonation mode was not observed for the $75 \%$ BR. Fast-flame propagation was observed for tests with initial pressures less than or equal to $35 \mathrm{kPa}$.

A comparison of quasi-detonation propagation velocities in the square and cylindrical channels showed that quasi-detonations in the square channel propagated at a higher average velocity than in the cylindrical channel. The larger velocity deficit (below the theoretical CJ detonation velocity) in the round tube is a result of the higher degree of diffraction that the wave undergoes. Diffraction over the fence-type obstacles in the square channel only occurs in the vertical direction representing a $2 \mathrm{D}$ diffraction, whereas in the round tube, diffraction occurs around the circumference of the orifice plate, representing a 3D diffraction. The lower propagation velocity in the round tube is a result of the $3 \mathrm{D}$ diffraction (and related higher curvature of the detonation wave), which caused the detonation wave to decouple more quickly. 
A final interesting observation was that the critical tube diameter, established in literature as $d_{\mathrm{c}} / \lambda \approx$ 13 in a round tube [33] [34] [36], may not be applicable to these types of experiments. For the 50\% BR tests, a continuous detonation (confirmed by uniform detonation cells on centreline soot foils) was only observed at initial pressures of $50 \mathrm{kPa}$ or higher (corresponding to a $\mathrm{d} / \lambda$ of 25 , instead of 13 ). For the $75 \%$ BR tests, a continuous detonation was not observed, even though a test pressure of $60 \mathrm{kPa}$ corresponds to a d/ $\lambda$ of 27 . Critical tube diameter studies rely on the transmission of a planar detonation wave, but the highspeed video indicated that in general a planar detonation did not propagate through the orifice plate.

This study provided a better understanding of detonation propagation in a round tube equipped with repeating orifice plates. The use of transparent soot foils, similar to the ones employed in [9], through which video is recorded would facilitate a better understanding of the detonation structure. Simultaneous highspeed photography and foils would reduce or eliminate uncertainty in analyzing the shock-flame structure recorded in the video to the cellular structure recorded on the foil at a different location. Installing pressure transducers or photo-diodes on the cylindrical channel (as done in steel tubes) would allow the combustion front to be tracked independent of the video images. 


\section{References}

[1] E. Mallard and H. Le Chatelier, "Recherches Experimentales et Theoriques sur la Combustion des Melanges Gaseoux Explosifs," Ann Mines, vol. 8, no. 4, pp. 274-568, 1883.

[2] G. Ciccarelli and S. Dorofeev, "Flame acceleration and transition to detonation in ducts," Progress in Energy and Combustion Science, vol. 34, pp. 499-550, 2008.

[3] W. Chapman and R. Wheeler, "The propagation of flame in mixtures of methane and air," Journal of the Chemical Society, pp. 38-46, 1927.

[4] D. Lieberman and J. Lee, "Photographic Study of the Transition Between the Quasi-Detonation and Choking Regimes," in International Colloquim on the Dynamics of Explosions and Reactive Systems, 2001.

[5] O. Peraldi, R. Knystautas and J. Lee, "Criteria for transition to detonation in tubes," Symposium (International) on Combustion, vol. 21, no. 1, pp. 1629-1637, 1986.

[6] G. Ciccarelli and M. Cross, "On the propagation mechanism of a detonation wave in a round tube with orifice plates," Shock Waves, vol. 26, no. 5, pp. 587-597, 2016.

[7] A. Teodorczyk, J. Lee and R. Knystautas, "Propagation mechanism of quasi-detonations," Symposium (International) on Combustion, vol. 22, no. 1, pp. 1723-1731, 1989.

[8] M. Kellenberger and G. Ciccarelli, "Propagation mechanisms of supersonic combustion waves," Proceedings of the Combustion Institute, vol. 35, no. 2, pp. 2109-2116, 2015.

[9] M. Kellenberger and G. Ciccarelli, "Investigation of quasi-detonation propagation using simultaneous soot foil and Schlieren photography," in 25th International Colloquium on the Dynamics of Explosions and Reactive Systems, Leeds, 2015.

[10] L. Boeck, M. Kellenberger, G. Rainsford and G. Ciccarelli, "Simultaneous OH_PLIF and Schlieren imaging of flame acceleration in an obstacle-laden channel," Proceedings of the Combustion Institute, vol. 36, no. 2, pp. 2807-2814, 2016.

[11] M. Kuznetsov, V. Alekseev, A. Bezmelnitsyn, W. Breitung, S. Dorofeev, I. Matsukov, A. Veser and Y. Yankin, "Effect of obstacle geometry on behaviour of turbulent flames," Institut für Kern- und Energietechnik, Karlsruhe, 1999.

[12] D. Chapman, "On the rate of explosions in gases," Philisophical Magazine Series 5, vol. 47, no. 284, pp. 90-104, 1899. 
[13] E. Jouguet, "On the propagation of chemical reactions in gases," Journal des Mathematiques Pures et Appliquees, vol. 6, no. 1, pp. 347-425, 1905.

[14] W. Rankine, "On the thermodynamic theory of waves of finite longitudinal disturbance," Philosophical Transactions of the Royal Society of London, vol. 160, pp. 277-288, 1870.

[15] H. Hugoniot, "Memoire sur la propagation du mouvement dans les corps," Journal de L'Ecole Polytechnique, 1887.

[16] G. Ciccarelli, MECH 832: Combustion Course Notes, Kingston: Queen's University, 2016.

[17] Y. Zeldovich, "On the theory of the propagation of detonation in gaseous systems," National Advisory Committee for Aeronautics, Washington, DC, 1950.

[18] J. Von Neumann, "Progress report on the theory of detonation waves," National Defense Research Committee of the Office of Scientific Research and Development, 1942.

[19] W. Doring, "Über Detonationsvorgang in Gasen (On detonation processes in gases)," Annalen der Physik, vol. 43, pp. 421-436, 1943.

[20] S. Kao and J. Shepherd, "Numerical Solution Methods for Control Volume Explosions and ZND Detonation Structures," California Institute of Technology, Pasadena, 2008.

[21] J. Fay, "Two-dimensional gaseous detonations: velocity deficit," The Physics of Fluids, vol. 2, no. 3, pp. 283-289, 1959.

[22] D. White, "Turbulent structure of gaseous detonation," Physics of Fluids, vol. 4, no. 4, pp. 465-480, 1961.

[23] Y. Denisov and Y. Troshin, "On the mechanism of detonative combustion," Symposium (International) on Combustion, vol. 8, no. 1, pp. 660-610, 1961.

[24] J. Austin, F. Pintgen and J. Shepherd, "Reaction zones in highly unstable detonations," Proceedings of the Combustion Institute, vol. 30, no. 2, pp. 1849-1857, 2005.

[25] J. Lee, The Detonation Phenomenon, New York: Cambridge University Press, 2008.

[26] Y. Gao, B. Zhang, H. Ng and J. Lee, "An experimental investigation of detonation limit in hydrogen-oxygen-argon mixtures," International Journal of Hydrogen Energy, vol. 41, no. 14, pp. 6076-6083, 2016.

[27] J. Shepherd, "Detonation in gases," Proceedings of the Combustion Institute, vol. 32, no. 1, pp. 8398, 2009.

[28] C. Campbell and D. Woodhead, "The ignition of gases by an explosion wave I: carbon monoxide and carbon mixtures," Journal of Chemical Society, vol. 301, pp. 3010-3021, 1926. 
[29] J. Lee, G. Dupré, R. Knystautas and J. Lee, "Doppler interferometry study of unstable detonations," Shock Waves, vol. 5, no. 3, pp. 175-181, 1995.

[30] J. Lee, The Detonation Phenomenon, New York: Cambridge University Press, 2008.

[31] Y. Zeldovich, S. Kogarko and N. Simonov, "An experimental investigation of spherical detonation in gases," Soviet Physics Technical Physics, vol. 1, pp. 1689-1713, 1956.

[32] K. Shchelkin and Y. Troshin, "Non-stationary phenomena in the gaseous detonation front," Combustion and Flame, vol. 7, pp. 143-151, 1963.

[33] V. Mitrofanov and R. Soloukhin, "The diffraction of multifront detonation waves," Sov. Phys. Dokl., vol. 9, p. 1055, 1965.

[34] R. Knystautas, J. Lee and C. Guirao, "The critical tube diameter for detonation failure in hydrocarbon-air mixtures," Combustion and Flame, vol. 48, pp. 63-83, 1982.

[35] D. Edwards, G. Thomas and M. Nettleton, "The diffraction of a planar detonation wave at an abrupt area change," Journal of Fluid Mechanics, vol. 95, no. 1, pp. 79-96, 1979.

[36] Y. Liu, J. Lee and R. Knystautas, "Effect of geometry on the transmission of detonation through an orifice," Combustion and Flame, vol. 56, pp. 215-225, 1984.

[37] W. Benedick, R. Knystautas and J. Lee, "Large scale experiments on the transmission of fuel-air detonations from two-dimensional channels," in Progress in Astronautics and Aeronautics: Dynamics of Shock Waves, Explosions and Detonations, American Institute of Aeronautics and Astronautics, 1985, pp. 546-555.

[38] J. Meyer and P. O. A. Urtiew, "On the inadequacy of gasdynamics processes for triggering the transition to detonation," Combustion and Flame, vol. 14, pp. 13-20, 1970.

[39] H. Yamashita, J. Kasahara, Y. Sugiyama and A. Matsuo, "Visualization study of ignition modes behind bifurcated-reflected shock waves," Combustion and Flame, vol. 159, pp. 2954-2966, 2012.

[40] E. Pantow, M. Fischer and T. Kratzel, "Decoupling and recoupling of detonation waves associated with sudden expansion," Shock Waves, vol. 6, pp. 131-137, 1996.

[41] K. Shchelkin, "Influence of tube roughness on the formation and detonation propagation in gas," Journal of Experimental Physics, vol. 10, pp. 823-827, 1940.

[42] O. Peraldi, R. Knystautas and J. Lee, "Criteria for transition to detonation in tubes," Proceedings of the Combustion Institute, vol. 21, pp. 1629-1637, 1986.

[43] M. Cross, "Detonation hazard classification based on the critical orifice plate diameter for detonation propagation. M.ASc. Thesis," Queen's University, Kingston, 2015. 
[44] C. Johansen, Experimental and Numerical Investigation of Flame Acceleration in an Obstructed Channel. PhD thesis., Kingston: Queen's University, 2009.

[45] M. Kellenberger, Dense Particle Cloud Dispersion by a Shock Wave. M.ASc Thesis., Kingston: Queen's University, 2012.

[46] M. Kaneshige and J. J.E. Shepherd, "Detonation Database. Technical Report FM97-8," Graduate Aerospace Laboratories of the California Institute of Technology (GALCIT), Pasadena, 1997.

[47] R. Knystautas, J. Lee and C. Guirao, "The critical tube diameter for detonation failure in hydrocarbon-air mixtures," Combustion and Flame, vol. 48, no. 1, pp. 63-83, 1982.

[48] D. Goodwin, Cantera: An object-oriented software toolkit for chemical kinetics, thermodynamics, and transport processes, Pasadena: Caltech, 2017.

[49] S. Browne, J. Ziegler and J. Shepherd, "Numerical solution methods for shock and detonation jump conditions. GALCIT Report," Graduate Aerospace Laboratories of the California Institute of Technology, Pasadena, 2015.

[50] M. Kellenberger and G. Ciccarelli, "Propagation mechanisms of supersonic combustion waves," Proceedings of the Combustion Institute, vol. 35, no. 2, pp. 2109-2116, 2015. 


\section{Appendix A \\ Additional Video Footage}

An example of hot-spot ignition behind the flame front is provided for a $25 \mathrm{kPa}$ test in Figure 5.1 which was originally filmed at 300,000 fps, but shown at an effective frame rate of 150,000 fps. Between OP 12 and 13, ignition is initiated at a single point at the top of the tube immediately before OP 13 (indicated by an arrow in Image 3). The hot-spot spreads radially around the tube wall (as indicated by white arrows in Images $4-6$ ). Ignition occurs well behind the flame front, which has propagated through the $14^{\text {th }}$ orifice plate at this point. Later, ignition occurs between Orifice Plates 14 and 15. Ignition occurs on the tube wall near the bottom of the tube before OP 15 (indicated by the arrow in Image 12) and propagates around the circumference of the tube (shown by the arrows in Images 13 and 14). However, the hot-spot does not spread around the full circumference of the tube, as indicated by the absence of light in Image 15. Ignition is also observed along the top of the tube as is seen between OP 13 and 14, and OP 14 and 15 in Images 21 - 22. In the case of each of these ignition events, the flame front is at least one diameter ahead of the event. As the velocity of the ignited gas behind the flame front cannot be accurately measured in these tests, these cannot definitively be called detonations. However, it is possible they are detonations as seen in higher pressure tests, such as the one shown in Figure 5.1. 

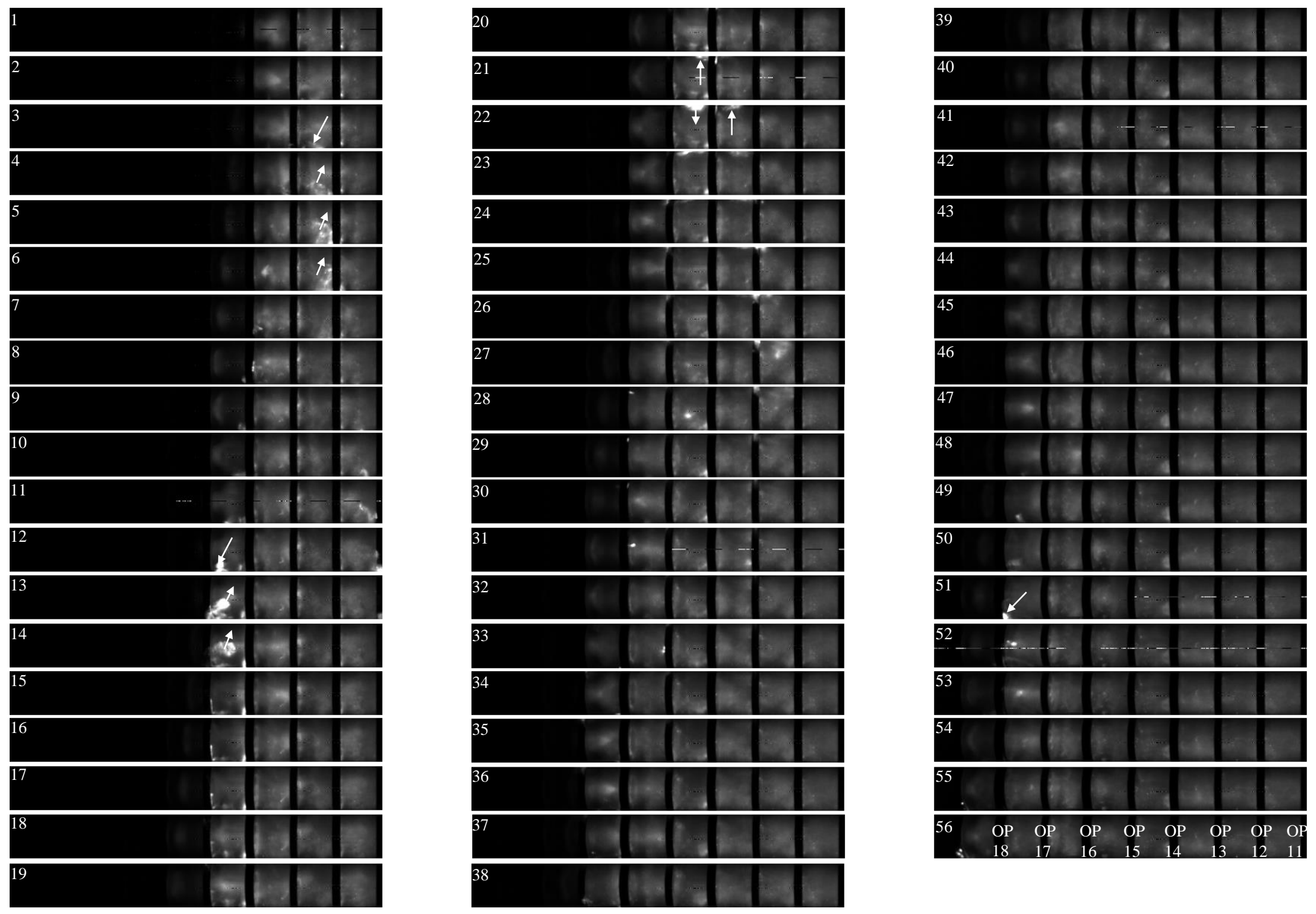

Figure 5.1: Test showing hot-spot ignition behind flame front for a $25.1 \mathrm{kPa}$ test with $75 \% \mathrm{BR}$, shown at 150,000 fps (Test 273 ) 130 


\section{Appendix B \\ Uncertainty Analysis}

This section summarizes the uncertainty associated with all quantitative measurements.

\section{Mixture Composition}

The uncertainty in the mixture composition comes from two sources: the uncertainty in making the mixture and the uncertainty in filling the combustion channel must be considered.

The uncertainty in creating the mixture can be calculated as follows. The pressure transducer in the combustion channel had a stated measurement accuracy of $0.25 \%$. Stoichiometric hydrogen-oxygen mixtures were made using the partial pressure method where $200 \mathrm{kPa}$ of hydrogen was combined with $100 \mathrm{kPa}$ of oxygen. The uncertainty associated with oxygen is \pm 0.25 $\mathrm{kPa}$ and $\pm 0.5 \mathrm{kPa}$ uncertainty associated with the hydrogen measurement. Assuming the worst case scenario (with the least amount of oxygen added and most amount of hydrogen added), the mixture composition is accurate to $0.1 \%$.

The pressure transducer in the combustion channel had a measurement accuracy of $0.25 \%$ corresponding to a maximum uncertainty of $\pm 0.15 \mathrm{kPa}$ at the highest pressures ( $50 \mathrm{kPa})$ and \pm 0.01 $\mathrm{kPa}$ at the lowest pressure $(4 \mathrm{kPa})$. The maximum allowable leak rate at vacuum in the combustion channel was measured to be $0.1 \mathrm{kPa}$ over 10 minutes or $0.01 \mathrm{kPa}$ per minute. As each test takes less than one minute from turning off the combustion channel vacuum pump, adding the mixture and initiating the test, the maximum quantity of air added to the mixture would be $0.01 \mathrm{kPa}$. Combining the two uncertainties yields an uncertainty of $\pm 0.16 \mathrm{kPa}$ for a $60 \mathrm{kPa}$ test and $\pm 0.02 \mathrm{kPa}$ for a $4 \mathrm{kPa}$ test.

Combing the two sources of error gives an overall uncertainty in mixture composition of $0.6 \%$ for a $4 \mathrm{kPa}$ test and $0.37 \%$ for a $60 \mathrm{kPa}$ pressure test. 


\section{Shock Time of Arrival Velocity Measurements}

The associated uncertainty in the shock-time-of-arrival measurements was determined based on the time of arrival recorded by the pressure transducers and the distance between them. According to the manufacturer's data sheet, the PCB pressure transducers have a response time of $\leq 1.0 \mu \mathrm{s}$, according to their data sheet and the DAQ card had a sampling rate of $3 \mathrm{MHz}$ for the tests. The estimated user error in determining the time of arrival is $\pm 0.25 \mu \mathrm{s}$. The associated error in the distance measurement is $\pm 0.05 "(0.13 \mathrm{~mm})$, which is the tolerance to which the combustion chamber was manufactured to. This gives an overall associated uncertainty in the combustion front velocity of $\pm 1 \mathrm{~m} / \mathrm{s}$.

\section{Video Velocity Measurements}

The uncertainty in the full-view, one-obstacle and centreline video velocity measurements was determined according to the following procedure. User error in manually identifying the combustion front is estimated to be \pm 1 pixel. The error in measure the pixel density, which was also determined manually, is estimated to be $\pm 3.45 \times 10^{-5} \mathrm{~m} /$ pixel. The associated error in the distance was estimated to be $\pm 0.13 \mathrm{~mm}$, which is the tolerance which the tube was manufactured to. The overall uncertainty associated with these measurements was $\pm 94 \mathrm{~m} / \mathrm{s}$.

The uncertainty for steady-state tests was based on the frame when the combustion wave impacted the orifice plate. The uncertainty associated with the time-of-arrival is the inverse of the frame rate equal to $\pm 1 / 300,000 \mathrm{~s}$. Depending on the initial pressure, the overall uncertainty ranged from $\pm 43 \mathrm{~m} / \mathrm{s}$ for a $4 \mathrm{kPa}$ test to $\pm 250 \mathrm{~m} / \mathrm{s}$ for a $60 \mathrm{kPa}$ test. 


\section{Appendix C \\ Detonation Cell Size Data}

Experiments were conducted by my colleague Julius Bertato as part of an undergraduate MECH 460 project measuring detonation cell size for stoichiometric hydrogen-oxygen mixtures. These experiments were completed in a $10 \mathrm{~cm}$ diameter Aluminum combustion tube filled with repeating orifice plates forming a blockage ratio of $43 \%$. Stoichiometric hydrogen-oxygen mixtures at initial pressures between 10 and $60 \mathrm{kPa}$ were ignited at one end of the combustion channel using a spark ignition system. Curved aluminum soot foils, similar to the ones described in Section 3.5, were installed at the far end of the $6.1 \mathrm{~m}$ tube. The detonation cell size for a range of cells was measured using calipers on the soot foil and averaged to determine the average detonation cell size. Due to the small cell sizes, for initial pressures greater than $30 \mathrm{kPa}$, the foils were photographed and MATLAB was used to digitally process the images.

A plot of cell size as a function of initial pressure is shown in Figure 5.2. The error bars indicate the standard error. 


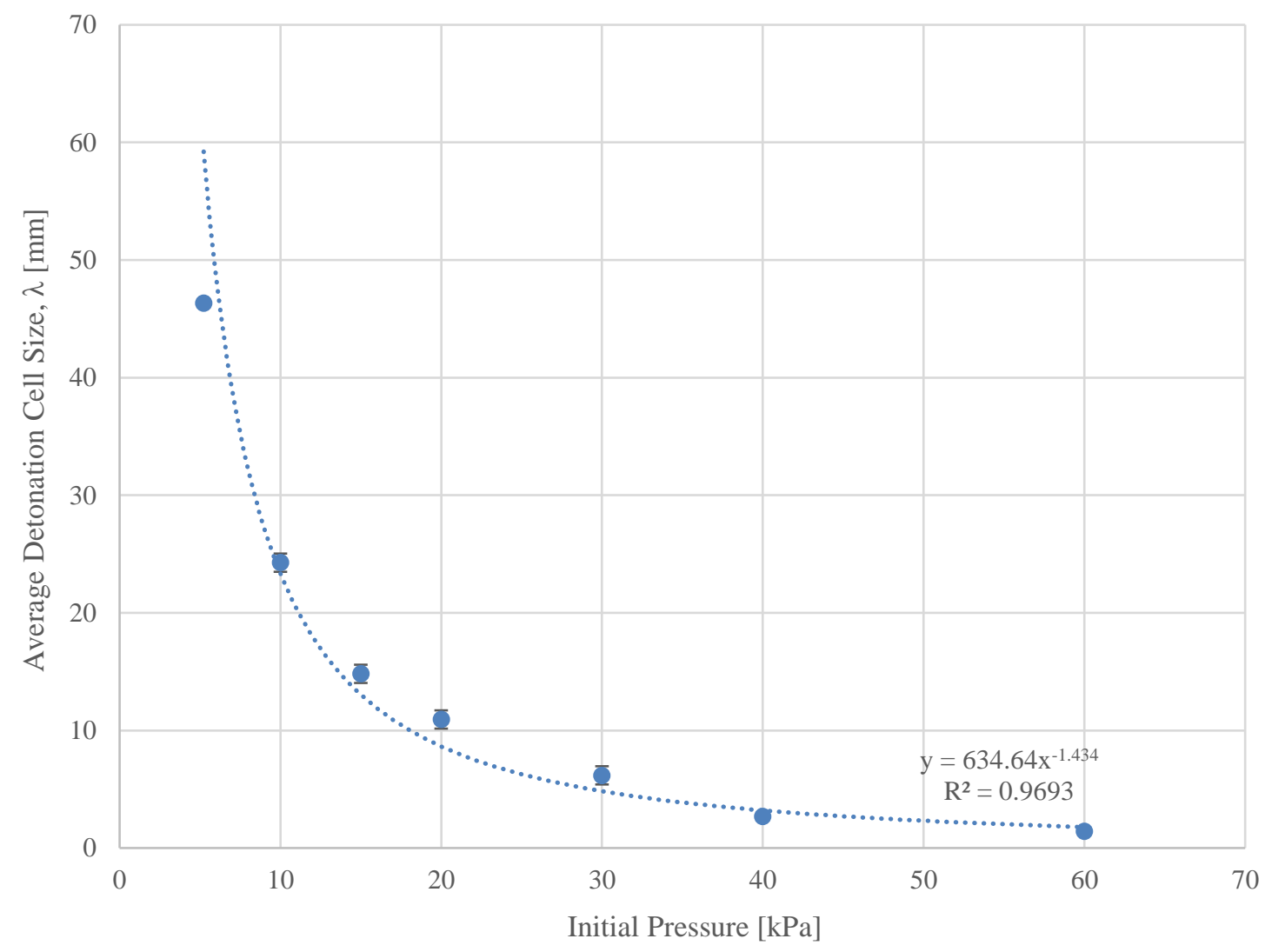

Figure 5.2: Plot of average detonation cell size as a function of initial pressure

As expected, the detonation cell size decreased as the initial pressure of the mixture was increased. The results were compared to cell size data obtained from the Graduate Aerospace Laboratories of the California Institute of Technology Detonation Database [46] and there was good agreement between the data. As tests were not conducted at initial pressures less than $10 \mathrm{kPa}$, cell size data obtained in [47] was added. The average cell size data was curve fitted in Excel to a power fit (which yielded the best $\mathrm{R}^{2}$ value of 0.9693 ). To ensure continuity in the data, the derived equation was used to calculate the average detonation cell size for all pressures tested.

Table 5.1 presents the calculated cell size data for all initial pressures tested in this thesis, as well as the calculated $d / \lambda$ value for both orifice diameters, $d$. 
Table 5.1: Calculated average detonation cell size and $d / \lambda$ ratio

\begin{tabular}{|c|c|c|c|}
\hline $\begin{array}{c}\text { Initial Pressure } \\
{[\mathrm{kPa}]}\end{array}$ & $\begin{array}{c}\text { Average } \\
\text { Detonation Cell } \\
\text { Size, } \lambda \\
{[\mathrm{mm}]}\end{array}$ & $\begin{array}{c}d / \lambda(d=5.33 \mathrm{~cm}) \\
{[-]}\end{array}$ & $\begin{array}{c}d / \lambda(d=3.81 \mathrm{~cm}) \\
{[-]}\end{array}$ \\
\hline 5 & 63.1 & 0.8 & 0.6 \\
\hline 6 & 48.6 & 1.1 & 0.8 \\
\hline 6.5 & 43.3 & 1.2 & 0.9 \\
\hline 7 & 39.0 & 1.4 & 1.0 \\
\hline 7.5 & 35.3 & 1.5 & 1.1 \\
\hline 8 & 32.2 & 1.7 & 1.2 \\
\hline 9 & 27.2 & 2.0 & 1.4 \\
\hline 10 & 23.4 & 2.2 & 1.6 \\
\hline 12 & 18.0 & 2.5 & 1.8 \\
\hline 15 & 13.1 & 3.6 & 2.6 \\
\hline 20 & 8.6 & 5 & 3 \\
\hline 25 & 6.3 & 8 & 6 \\
\hline 30 & 4.8 & 9 & 6 \\
\hline 35 & 3.9 & 14 & 10 \\
\hline 40 & 3.2 & 20 & 14 \\
\hline 45 & 2.7 & 21 & 15 \\
\hline 50 & 2.3 & 25 & 18 \\
\hline 55 & 2.0 & 29 & 21 \\
\hline 60 & 1.8 & 37 & 27 \\
\hline
\end{tabular}




\section{Appendix D}

\section{Calculated CJ Velocity and Speed of Sound}

The CJ detonation velocity and combustion products speed of sound calculations were completed using the chemical equilibrium code Cantera [48] was used in conjunction with the Shock and Detonation Toolbox [49]. These calculations were performed for stoichiometric hydrogen-oxygen mixtures at SATP and initial pressures between 5 and $65 \mathrm{kPa}$. The calculated data is summarized in Table 5.2.

Table 5.2: Calculated CJ Velocity and Products Speed of Sound

\begin{tabular}{|c|c|c|}
\hline Initial Pressure [kPa] & $\begin{array}{c}\text { CJ Detonation Velocity } \\
{[\mathbf{m} / \mathbf{s}]}\end{array}$ & $\begin{array}{c}\text { Combustion Products } \\
\text { Speed of Sound } \\
{[\mathbf{m} / \mathbf{s}]}\end{array}$ \\
\hline 5 & 1390 & 2675 \\
\hline 10 & 1402 & 2711 \\
\hline 15 & 1410 & 2733 \\
\hline 20 & 1415 & 2749 \\
\hline 25 & 1419 & 2761 \\
\hline 30 & 1422 & 2770 \\
\hline 35 & 1425 & 2779 \\
\hline 40 & 1428 & 2786 \\
\hline 45 & 1430 & 2792 \\
\hline 50 & 1432 & 2798 \\
\hline 55 & 1434 & 2803 \\
\hline 60 & 1435 & 2808 \\
\hline 65 & 1437 & 2812 \\
\hline
\end{tabular}

